



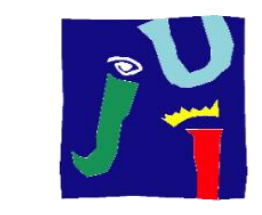

UNIVERSITAT JAUME•I
Programa de Doctorat en Ciències

Escola de Doctorat de la Universitat Jaume I

\section{Development of New Hybrid Materials Based on Graphene Functionalised with Molecular Complexes. Evaluation of Properties and Catalytic Applications}

Memòria presentada per David Ventura Espinosa per a optar al grau de doctor per la Universitat Jaume I

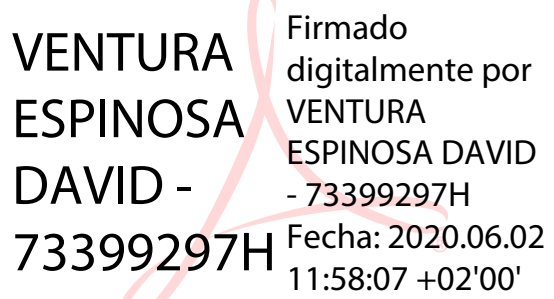

David Ventura Espinosa

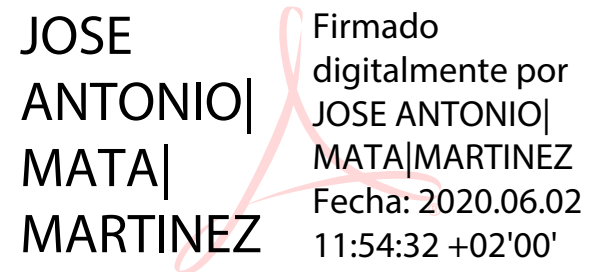

Supervisor: Jose A. Mata Martínez 

The present Doctoral Thesis has been carried out thanks to the "Programa de formación del profesorado universitario (FPU) (Reference: FPU15/03011)" financed by the Spanish Government.

The work developed in this Doctoral Thesis has been possible thanks to the financial support from the following research projects:

- CTQ2015-69153-C2-2-R

- AICO/2015/039

- P1.1B2015-09

- UJI-B2018-23

- RTI2018-098237-B-C22
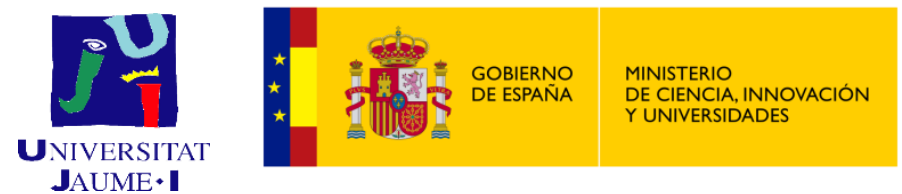



\section{Dr. Jose Antonio Mata Martínez, professor titular de l'àrea de Química Inorgànica pertanyent al Departament de Química Inorgànica i Orgànica de la Universitat Jaume I}

Certifica: Que la Tesi Doctoral amb títol "Development of new hybrid materials based on graphene functionalised with molecular complexes. Evaluation of properties and catalytic applications" ha sigut desenvolupada sota la seua direcció en l'àrea de Química Inorgànica del Departament de Química Inorgànica i Orgànica de la Universitat Jaume I per David Ventura Espinosa.

Castelló de la Plana, Juny de 2020

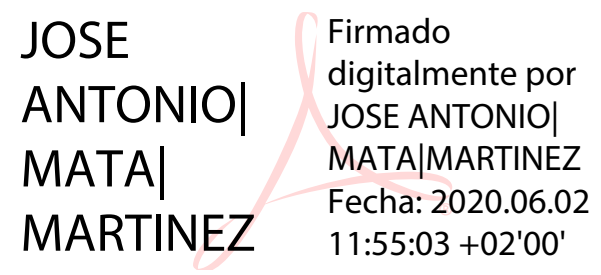

Sign. Dr. Jose A. Mata Martínez 



\section{AGRAIIMENTS}

Corria juliol de 2013 quan un alumne que estava acabant el tercer curs del grau es va plantar amb incertesa i veu tremolosa a la porta contigua al que avui és el meu despatx, per tal de veure que era això de la recerca i com funcionava. Ara, quasi set anys després, em veig front a aquest full en blanc, tractant d'expressar en unes poques línies el meu agraïment a totes aquelles persones que han estat presents al Ilarg d'aquesta etapa. Un bon resum per a aquells que no voleu seguir llegint podria ser: "Quina sort va tindre David!".

Els primers agraïments no poden ser per un altre que el principal responsable de que aquest treball sigui avui tangible. Gràcies Jose per tots els consells, per la teva inesgotable paciència i per l'ajuda constant en tot el que estava al teu abast tots i cadascun dels dies. Aquesta tesi és en gran part teva.

Gràcies també als meus companys de laboratori. Als "jefes": a Eduardo, per permetrem en primera instància incorporar-me a aquest grup; a Maca, sens la qual no aguantaríem sols ni dos dies; a Susana, a qui sempre podies recórrer quan no sabies on era alguna cosa; i a Goyo, per totes les batalletes arreu del món que ens conta, pel seu altruisme que fa que deixi el que ell està fent per a centrar-se en ajudar-te i per estar sempre dispost a tindre discussions que pot ser et fan replantejar-te fins el teu propi nom, però de les que sempre aprens alguna cosa.

Als doctors que han anat deixant el grup: Sara (gràcies per tota l'ajuda inicial i la paciència que vas tindre amb mi), Candela, Sergio, Hugo, Sheila i en especial a Carmen amb qui vaig començar a la vegada i em va adoptar des de l'inici. Als membres no doctors del grup QOMCAT, Victor (quasi 10 anys seguint el mateix camí), Ana (mai faltarà xocolata a la nostra despensa particular), Chiara i Dani (eixe riure que tant s'apega i eixa curiositat sense fi que tant fascinant resulta). Menció especial també per a Mapi, per portar eixa alegria diària al laboratori el temps que va passar al grup, i el nouvingut Andrés, un plaer compartir laboratori i festes amb tu! Gràcies també a la professora Sílvia Díez-Gonzàlez per acceptar-me al seu grup i permetre'm viure una gran experiència de tres mesos a Londres. Gràcies a tots aquells que han passat pel laboratori i tant ens han ensenyat: George, Ian, Tobias, Max, Heidar... i a Iván Sorribes, per tots els dinars i tot el que s'aprèn treballant amb tu. 
Als tècnics del Servei Central d'Instrumentació Científica, en especial a Cristian, Jose, Laura i Maruxa; i a la secció saragossana, Santi i Miguel, per tota l'ajuda durant la tesi i per l'hospitalitat durant les meves visites allí.

Als membres dels altres grups de QIO. Per la disponibilitat per ajudar-me les no poques vegades que anava a buscar algun reactiu i per recordar-me de tant en tant que hi ha món més enllà de les quatre parets de l'edifici d'investigació I, pels dinars a la cantina o als sentits $\mathrm{i}$ per tots els esdeveniments sòcio-culturals que hem anat organitzant aquests anys. En especial gràcies a aquells que han estat de principi a fi: Adriana (alegria contagiosa desde el minuto cero), Ana (personas bonitas que la vida te pone en el camino) i a Iván, per ajudar-me a mantindre'm en forma, per les bones estones a I'UJI i també fora; i per les escapades a Elx, Mallorca, Croàcia, Saragossa o Logronyo.

A la meva família Nulera, perquè les coses són encara més increïbles quan es té uns amics com vosaltres. Gràcies per tots els bons moments, viatges, festes o simplement cerveses a vora mar on podem des de filosofar, tractar d'arreglar el món, organitzar el pròxim festival o tan sols desfruitar del silenci en bona companyia. Però també gràcies per ser uns grans amics en els moments de més dubtes.

Als meus amics de Castelló, per acollir-me amb eixa calidesa tant especial quan no coneixíeu res de mi, sou molt molt grans!

Al ministeri d'educació, pel contracte FPU que m’ha permès desenvolupar aquesta tesi doctoral.

I per suposat, gràcies als meus pares i al meu germà. Pel suport incondicional, la paciència infinita i per ensenyar-me el camí però també per deixar que m'enganyés quan calia; i per generar el millor entorn possible per a nosaltres. 
Los siguientes artículos han sido publicados como consecuencia del trabajo recogido en la presente tesis doctoral:

- D. Ventura-Espinosa, C. Vicent, M. Baya, J. A. Mata. "Ruthenium Molecular Complexes Immobilized on Graphene as Active Catalysts for the Synthesis of Carboxylic Acids from Alcohol Dehydrogenation." Catal. Sci. Technol. 2016, 6, 8024-8035.

- D. Ventura-Espinosa, A. Marza-Beltran, J. A. Mata. "Catalytic Hydrogen Production by Ruthenium Complexes from the Conversion of Primary Amines to Nitriles: Potential Application as a Liquid Organic Hydrogen Carrier." Chem. Eur. J. 2016, 22, 17758-17766.

- D. Ventura-Espinosa, A. Carretero-Cerdán, M. Baya, H. García and J. A. Mata. "Catalytic Dehydrogenative Coupling of Hydrosilanes with Alcohols for the Production of Hydrogen On-demand: Application of the Silane/Alcohol Pair as a Liquid Organic Hydrogen Carrier." Chem. Eur. J. 2017, 23, 10815-10821.

- D. Ventura-Espinosa, S. Sabater, J. A. Mata. "Enhancement of Gold Catalytic Activity and Stability by Immobilization on the Surface of Graphene." J. Catal. 2017, 352, 498-504.

- D. Ventura-Espinosa, S. Sabater, A. Carretero-Cerdán, M. Baya, J. A. Mata. "High Production of Hydrogen on Demand from Silanes Catalyzed by Iridium Complexes as a Versatile Hydrogen Storage System." ACS Catal. 2018, 8, 2558-2566.

- D. Ventura-Espinosa, S. Martín, J. A. Mata. "The non-Innocent Role of Graphene in the Formation/Immobilization of Ultra-Small Gold Nanoparticles Functionalized with N-heterocyclic Carbene Ligands." J. Catal. 2019, 375, 419-426. 
Además de los anteriores, durante el transcurso de la tesis han sido publicados los siguientes trabajos:

- D. Ventura-Espinosa, J. A. Mata. "Multiple-Metal (De-)HydrogenationCatalysed Processes." Eur. J. Inorg. Chem. 2016, 2016, 2667-2675.

- R. Torres-Mendieta, D. Ventura-Espinosa, S. Sabater, J. Lancis, G. MinguezVega, J. A. Mata. "In Situ Decoration of Graphene Sheets with Gold Nanoparticles Synthetized by Pulsed Laser Ablation in Liquids." Sci. Rep. 2016, 6, 30478.

- C. Barreneche, R. Mondragón, D. Ventura-Espinosa, S. Sabater, J. A. Mata, L. F. Cabeza, A. I. Fernández, J. Enrique Julia. "Influence of Nanoparticle Morphology and its Dispersion Ability Regarding Thermal Properties of Water Used as Phase Change Material." Appl. Therm. Eng. 2018, 128, 121126.

- B. Zelenay, M. Besora, Z. Monasterio, D. Ventura-Espinosa, A. J. P. White, F. Maseras, S. Díez-González. "Copper-Mediated Reduction of Azides under Seemingly Oxidising Conditions: Catalytic and Computational Studies." Catal. Sci. Technol. 2018, 8, 5763-5773.

- A. Mollar-Cuni, D. Ventura-Espinosa, S. Martín, A. Mayoral, P. Borja, J. A. Mata. "Stabilization of Nanoparticles Produced by Hydrogenation of Palladium N-Heterocyclic Carbene Complexes on the Surface of Graphene and Implications in Catalysis." ACS Omega 2018, 3, 15217-15228.

- P. Ballestin, D. Ventura-Espinosa, S. Martín, A. Caballero, J. A. Mata, P. J. Pérez. "Improving Catalyst Activity in Hydrocarbon Functionalization by Remote Pyrene-Graphene Stacking." Chem. Eur. J. 2019, 25, 9534-9539. 
Index

Nomenclature

vi

List of abbreviations

vi

CHAPTER 1: General Introduction

1.1 RELEVANCE OF CATALYSIS 3

1.2 RECYCLING STRATEGIES FOR HOMOGENEOUS CATALYSTS 4

1.2.1 Homogeneous catalysis/heterogeneous separation 5

1.2.2 Immobilised homogeneous catalysis 7

1.3 CHEMICALLY DERIVED GRAPHENES AS SUPPORTS 10

1.3.1 Preparation of chemically derived graphenes 11

1.3.2 Covalent and non-covalent immobilisation of molecular complexes on chemically derived graphenes 12

$\begin{array}{lll}\text { 1.3.3 Precedents in our research group } & 15\end{array}$

$\begin{array}{lll}1.4 & 19\end{array}$

$\begin{array}{lll}1.5 & \text { REFERENCES } & 20\end{array}$

CHAPTER 2: Ru-NHC-rGO as Reusable Catalysts for the Dehydrogenation of Alcohols in Aqueous Media 25

$\begin{array}{lll}2.1 & 27\end{array}$

2.2 RESULTS AND DISCUSSION 34

2.2.1 Synthesis of ruthenium catalysts and hybrid ruthenium materials 34

2.2.2 Catalytic studies: acceptorless alcohol oxidation 37

$\begin{array}{lll}2.3 & \text { CONCLUSIONS } & 50\end{array}$

$2.4 \quad$ REFERENCES 51 
CHAPTER 3: Nitrile Synthesis by Amine Dehydrogenation

$\begin{array}{lll}3.1 & 57\end{array}$

$\begin{array}{lll}3.2 & \text { RESULTS AND DISCUSSION } & 61\end{array}$

3.2.1 Synthesis of ruthenium complexes 61

3.2.2 Catalytic dehydrogenation of primary amines 61

$\begin{array}{lll}3.3 & \text { CONCLUSIONS } & 74\end{array}$

$\begin{array}{lll}3.4 & \text { REFERENCES } & 75\end{array}$

CHAPTER 4: Hydrogen Production on-Demand from the Coupling of Alcohols and Silanes Catalysed by Ruthenium and Iridium Complexes Grafted onto Reduced Graphene Oxide

$\begin{array}{lll}4.1 & 7 N T R O D U C T I O N & 79\end{array}$

4.1.1 Liquid organic hydrogen carriers 79

4.1.2 The silane-alcohol pair $\quad 85$

4.1.2.1 Reduction of silyl ethers 87

$\begin{array}{lll}4.2 & \text { RESULTS AND DISCUSSION } & 91\end{array}$

4.2.1 Synthesis and characterisation of an Iridium complex bearing a pyrene tag and immobilisation onto graphene

4.2.2 Hydrogen production from the coupling between silanes and alcohols catalysed by ruthenium complexes anchored onto rGO 93

4.2.3 Hydrogen production from the coupling between silanes and alcohols catalysed by iridium complexes anchored onto rGO 102 4.2.4 Mechanistic studies 106

$\begin{array}{lll}4.3 & \text { CONCLUSIONS } & 111\end{array}$

$\begin{array}{lll}4.4 & \text { REFERENCES } & 112\end{array}$ 
CHAPTER 5: Gold Catalysis at the Surface of Graphene

5.1 INTRODUCTION

5.1.1 Immobilisation of gold molecular complexes

5.1.2 Generation and immobilisation of metal nanoparticles. Applications in catalysis

5.2 RESULTS AND DISCUSSION

5.2.1 Molecular complexes anchored onto reduced graphene oxide

132

5.2.1.1 Synthesis and characterisation of ligand precursor $\mathbf{H}$ and gold complex $\mathbf{1 0 H}$

5.2.1.2 Immobilisation of $9 \mathrm{C}$ and $\mathbf{1 0 H}$ onto $\mathrm{rGO}$

133

5.2.1.3 Catalytic properties of gold molecular complexes and the related hybrid materials

5.2.2 NHC functionalised gold nanoparticles anchored on graphene

5.2.2.1 Synthesis and characterisation of ligand precursor I and metal complex 11I

5.2.2.2 Direct generation and immobilisation of NHC-capped Au-NPs onto rGO

5.2.2.3 Catalytic properties of $12 \mathrm{I}, \mathbf{1 2 I}-\mathrm{rGO}-\mathrm{NPs}$ and $\mathrm{HAuCl}_{4^{-}}$ rGO-NPs

5.3 CONCLUSIONS

5.4 REFERENCES

158

CHAPTER 6: Experimental Section

6.1 ANALYTICAL TECHNIQUES

6.2 SYNTHESIS AND CHARACTERISATION 166

6.2.1 Synthesis and characterisation of ligand precursors 
6.2.2 Synthesis and characterisation of metal complexes

6.2.3 Synthesis and characterisation of hybrid M-rGO materials 174

6.3 CATALYTIC EXPERIMENTS 192

6.3.1 Dehydrogentative oxidation of alcohols 192

6.3.2 Dehydrogenation of amines 193

6.3.3 Dehydrogenative coupling between silanes and alcohols 194

6.3.4 Intramolecular hydroamination 198

6.3.5 Hydration of alkynes 199

$\begin{array}{lll}6.4 & \text { DFT STUDIES } & 200\end{array}$

$\begin{array}{lll}6.5 & \text { X-RAY DIFFRACTION } & 201\end{array}$

6.6 REFERENCES 205

$\begin{array}{ll}\text { CHAPTER } 7 \text { Resumen } & 207\end{array}$

$\begin{array}{lll}7.1 & 209\end{array}$

$\begin{array}{lll}7.2 & \text { OBJETIVOS } & 213\end{array}$

$\begin{array}{lll}7.3 & \text { RESULTADOS Y DISCUSIÓN } & 214\end{array}$

7.3.1 Deshidrogenación catalítica de alcoholes 214

7.3.2 Deshidrogenación catalítica de aminas para la síntesis de nitrilos

7.3.3 Generación de hidrógeno a partir de la reacción de acoplamiento de alcoholes e hidrosilanos

7.3.3.1 Acoplamiento de silanos y alcoholes catalizado por especies de rutenio

7.3.3.2 Acoplamiento de silanos y alcoholes catalizado por especies de iridio 
7.3.4 Catalizadores de oro en la superficie de grafeno

7.3.4.1 Complejos moleculares de oro anclados sobre rGO 225

7.3.4.2 Nanopartículas de oro funcionalizadas con ligandos

$\mathrm{NHC}$ ancladas sobre la superficie de rGO

228

$\begin{array}{lll}7.4 & \text { CONCLUSIONES } & 232\end{array}$

$\begin{array}{lll}7.5 & \text { REFERENCIAS } & 234\end{array}$ 


\section{Nomenclature}

The nomenclature used to name the compounds of this works is:

- Imidazolium salts that serve as ligand precursors: letters of the alphabet (AI).

- Metal complexes with NHC ligands: a number followed by the letter of the coordinated ligand. The complexes have been sorted in the order of appearance in the text.

- Hybrid materials: the name of the grafted complex followed by -rGo. In the case of hybrid materials based on nanoparticles the suffix -NPs is added at the end.

\section{List of Abbreviations}

$\begin{array}{ll}\Delta & \text { Refluxing temperature } \\ \eta & \text { Ligand hapticity } \\ \lambda & \text { Waverlength } \\ 9 \text {-BBN } & \text { 9-borabicyclo [3.3.1] nonane } \\ \text { ADH } & \text { Acceptorless de-hydrogenation } \\ \text { a.m.u } & \text { Atomic Mass Unit } \\ \text { Au-NPs } & \text { Gold nanoparticles } \\ n B u & n \text {-butyl } \\ t \text { BuOH } & \text { tert-butanol } \\ t \text { BuOK } & \text { Potassium tert-butoxide } \\ \text { BTMAH } & \text { Benzyltrimethylamonium hydroxide } \\ \text { cat } & \text { Catalyst } \\ \text { CDGs } & \text { Chemically derived graphenes } \\ \text { CID } & \text { Collision Induced Dissociation } \\ p \text {-cym } & p \text {-cymene } \equiv \text { 1-methyl-4-(iso-propyl)benzene } \\ \text { DBT } & \text { Dibenzyltoluene } \\ \text { DFT } & \text { Density Functional Theory } \\ \text { DME } & 1,2 \text { dimethoxyethane }\end{array}$




\begin{tabular}{|c|c|}
\hline DMF & Dimethylformamide \\
\hline Dipp & 2,6-diisopropyl phenyl \\
\hline EDS & Energy Dispersive X-Ray Spectroscopy \\
\hline $\mathrm{Eq}$ & Equivalent \\
\hline ESI-MS & Electrospray lonization Mass Spectrometry \\
\hline EtOAc & Ethyl acetate \\
\hline $\mathrm{EtOH}$ & Ethanol \\
\hline FA & Formic acid \\
\hline FID & Flame Ion Detector \\
\hline GC & Gas Chromatography \\
\hline GO & Graphene Oxide \\
\hline h & Hour \\
\hline HBpin & 4,4,5,5- tetramethyl-1,3,2-dioxoborolane \\
\hline HRTEM & High Resolution Transmission Electron Microscopy \\
\hline HSC & Hydrogen storage capacity \\
\hline HRMS & High Resolution Mass Spectrometry \\
\hline HT & Hydrogen transfer \\
\hline ICP-MS & Inductively Coupled Plasma-Mass Spectrometry \\
\hline L & Ligand \\
\hline LOHC & Liquid organic hydrogen carrier \\
\hline Hp-LOHC & Hydrogen poor molecule of a $\mathrm{LOHC}$ \\
\hline $\mathrm{Hr}-\mathrm{LOHC}$ & Hydrogen rich molecule of a $\mathrm{LOHC}$ \\
\hline $\mathrm{M}-\mathrm{C}$ & Metal-carbon \\
\hline $\mathrm{MCH}$ & Methylcyclohexane \\
\hline $\mathrm{Me}$ & Methyl \\
\hline $\mathrm{MeCN}$ & Acetonitrile \\
\hline mes & 2,4,6-trimethylbenzene \\
\hline $\min$ & Minute \\
\hline MS & Mass Spectrometry \\
\hline $\mathrm{m} / \mathrm{z}$ & Mass to charge ratio \\
\hline $\mathrm{NaBAr}_{4}^{\mathrm{F}}$ & Sodium tetrakis-[3,5-bis(trifluoromethyl)phenyl] borate \\
\hline NEC & $\mathrm{N}$-ethylcarbazole \\
\hline
\end{tabular}




\begin{tabular}{|c|c|c|}
\hline \multirow{2}{*}{\multicolumn{2}{|c|}{$\mathrm{NHC}$}} & N-heterocyclic carbene \\
\hline & & Nuclear Magnetic Resonance \\
\hline & $\delta$ & Chemical shift \\
\hline & $d$ & Doublet \\
\hline & J & Coupling constant \\
\hline & $\mathrm{m}$ & Multiplet \\
\hline & ppm & Parts per million \\
\hline & s & Singlet \\
\hline & $\mathrm{t}$ & Triplet \\
\hline NPs & & Nanoparticles \\
\hline Otf & & Trifluotomethanesulfonate \\
\hline PVP & & Poly(4-vinylpyridine) \\
\hline iPrOH & & Isopropanol \\
\hline$n \mathrm{PrOH}$ & & 1-propanol \\
\hline pHDBT & & Perhydro-dibenzyltoluene \\
\hline pHNEC & & Perhydro-N-ethylcarbazole \\
\hline rGO & & reduced Graphene Oxide \\
\hline RT & & Room temperature \\
\hline SBA-15 & & Santa Barbara amorphous silica-type material \\
\hline SEM & & Scanning Electron Microscopy \\
\hline SMR & & Steam methane reforming \\
\hline TCD & & Thermal Conductivity Detector \\
\hline THF & & Tetrahydrofuran \\
\hline TON & & Turnover number \\
\hline TOF & & Turnover frequency \\
\hline TOL & & Toluene \\
\hline US & & Ultrasounds \\
\hline UV-Vis & & Ultraviolet-Visible \\
\hline WE & & Water electrolysis \\
\hline wt \% & & Percentage in weight \\
\hline XANES & & X-Ray Absorption Near Edge Structure \\
\hline XPS & & X-Ray Photoelectron Spectroscopy \\
\hline
\end{tabular}


CHAPTER 1

General Introduction 



\subsection{RELEVANCE OF CATALYSIS}

It is undeniable that catalysis plays a key role in our daily life. From fuels to pharmaceutical drugs, almost any commodity chemical that we can think of has at least one catalytic step in its manufacture. ${ }^{[1]}$ By definition a catalyst is "A substance that increases the rate of a reaction without consumption".

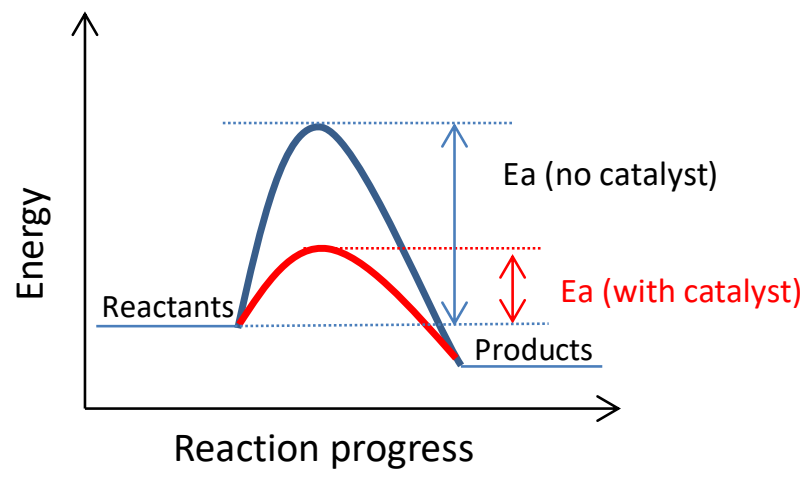

Figure 1.1 Differences in the energy profile for a catalysed and a non-catalysed version of the same reaction

A catalyst does not modify the relative free energy of reactants and products, but provides an alternative reaction pathway with a lower energetic transition state (Figure 1.1). In other words, a catalyst does not affect the thermodynamics of a transformation, but it has a dramatic effect on the kinetics. Thus, the use of catalysts opens a gate to new chemical reactivity that would not be possible in the absence of a catalyst.

Excluding enzymes, catalysts are usually divided in two main groups, homogeneous and heterogeneous. In the traditional definition of homogeneous catalysis, the catalyst is in the same phase than the reactants. In this way, the contact of the catalytic active centres with the substrates is easy and, as a result, homogeneous catalysts are more active than heterogeneous. In addition, homogeneous catalysts are usually well-defined molecular entities, which facilitate the study of the reaction mechanism. Mechanistic studies provide new information for the design of more active and selective catalysts. The main drawbacks of homogeneous catalysts are the difficult and/or costly separation of the catalyst from the reaction media and sometimes the poor stability, which implies the use of higher catalyst loadings. This is an issue for large scale applications, especially when the final product is a pharmaceutical because metal contamination must be avoided. 
In spite of the attractive features of homogeneous catalysis, today around $90 \%$ of industrial catalytic processes make use of heterogeneous catalysts. ${ }^{[2]}$ In heterogeneous catalysis the catalyst and the reactants are in different phases, so the catalyst can be easily separated at the end of the reaction. Typical heterogeneous catalysts are metal oxides or supported metal nanoparticles. The use of a biphasic system usually makes more difficult the access of reactants to the active centres, thus affecting the rate of the reaction. The mass transfer problems require the use of harsher reaction conditions such as increased pressures or temperatures that lead to more energy intensive processes. Nevertheless, the solid nature of heterogeneous catalysts, high stability and easy separation constitute the basis for its good acceptance from the chemical industry.

However, in the look of more sustainable processes, an important part of the academic world has put a considerable effort in combining the benefits of homogeneous and heterogeneous catalysis. The development of novel strategies to efficiently recover homogeneous catalysts will bring a huge benefit for both, industry and society. The use of recyclable homogeneous catalysts will allow the reduction of reaction times and energy consumption, thus increasing the productivity and reducing costs. In addition, the employment of more selective and recoverable homogeneous catalysts will reduce the generation of waste sideproducts, which is also convenient from an environmental point of view.

\subsection{RECYCLING STRATEGIES FOR HOMOGENEOUS CATALYSTS}

Over the last years, different approaches have been developed for the recycling and reutilisation of homogeneous catalysts. These strategies can be divided in two main groups (Scheme 1.1). 


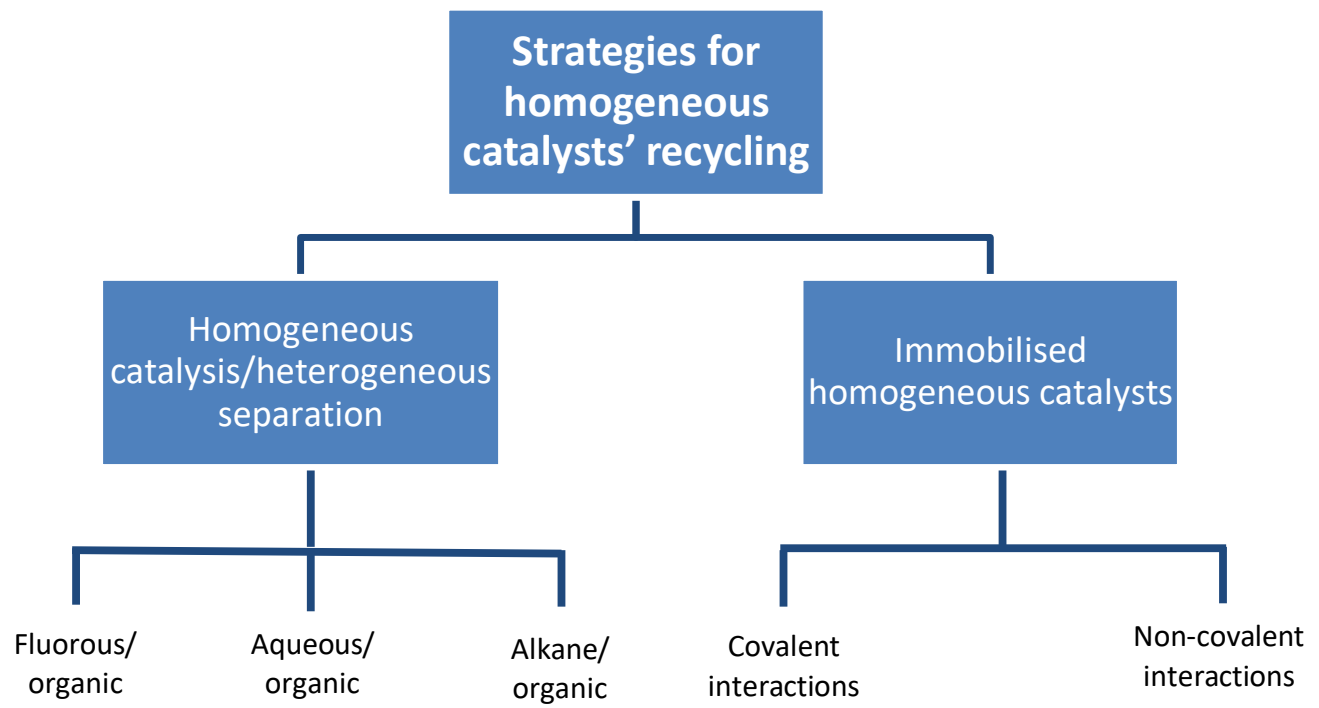

Scheme 1.1 Strategies for homogeneous catalysts' recycling

\subsubsection{Homogeneous catalysis/heterogeneous separation}

The first group includes catalytic systems where the reaction takes place under homogeneous conditions and then the separation in different phases allows catalyst recovery. These systems often use mixtures of water and organic solvents, although other combinations are possible (Scheme 1.1).$^{[3,4]}$ For this purpose, the catalyst is previously functionalised with an organic group that determines its preferential solubility in one of the solvents. Commonly, a polar group is introduced to favour the solubility of the catalyst in water. The most common hydrophilic groups used are sulfonates, although other functionalities have been explored. ${ }^{[5]}$ The catalytic reactions are then performed in water where both, the reactants and the catalyst, are soluble. After the reaction, an organic solvent immiscible with water (e.g. dichloromethane, toluene, ethyl acetate or diethyl ether) is added, which causes phase separation. The organic products are preferentially displaced to the organic solvent, while the water-soluble catalyst remains in the aqueous phase. Simple decantation enables the separation of the products from the catalyst. Next, the aqueous solution of the catalyst can be fed with more reactants and start a new cycle (Figure 1.2). 


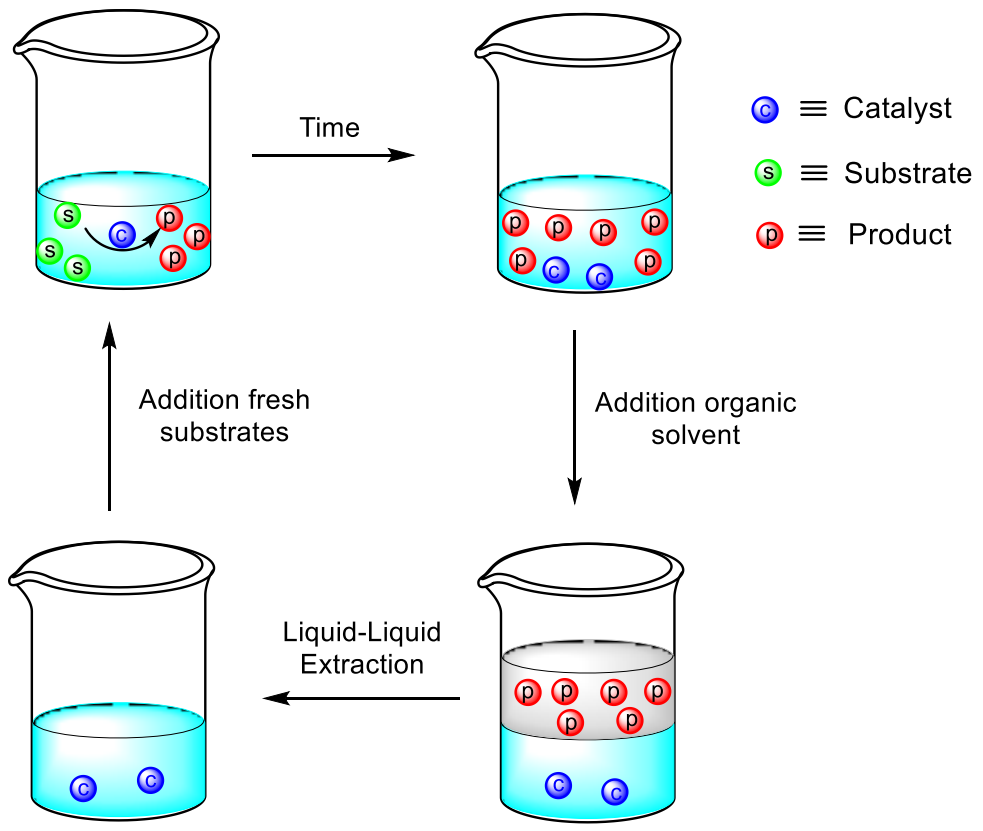

Figure 1.2 Catalyst recycling based on the separation of different phases

In early 2000s, Sheldon and co-workers reported the first efficient homogeneous catalyst for the oxidation alcohols in water media. ${ }^{[6]}$ The catalytic system is based on a palladium (II) complex that features a phenanthroline ligand functionalised with a sulfonate moiety (Figure 1.3, top). The presence of this functional group renders a water-soluble catalyst that can oxidise a wide range of primary and secondary alcohols to aldehydes and ketones. In this seminal work the authors proved the high stability of the catalyst by recycling it up to six runs with consistent high yields. Years later, Fujita and Yamaguchi reported an improved water-soluble cationic iridium catalyst for the dehydrogenation of alcohols (Figure 1.3, bottom). ${ }^{[7]}$ The main advantage of this system is that the oxidation of alcohols is produced through dehydrogenation, avoiding the use of high pressures of air or $\mathrm{O}_{2}$. In this way, the only generated by-product is hydrogen, which has potential applications as fuel. ${ }^{[8]}$ After the reaction, the products were extracted with hexane and the catalystcontaining aqueous solution could be reused up to eight times without any decrease in activity. 


$$
\sum_{-\mathrm{OH}}^{\stackrel{[\mathrm{Pd}](0.25 \mathrm{~mol} \%)}{\mathrm{NaOAc}, 30 \text { bar } \mathrm{O}_{2}}}
$$

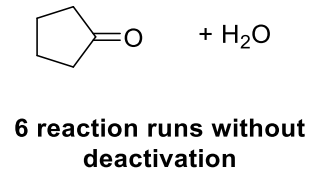

deactivation

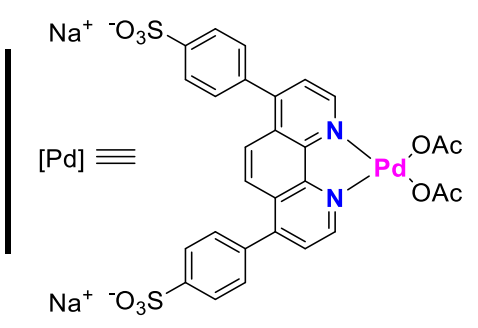

$[\mathrm{Ir}]=[\mathrm{OTf}]_{2}$

Figure 1.3 Recyclable, water-soluble systems reported by Sheldon (top) and Yamaguchi (bottom) for the oxidation of alcohols

\subsubsection{Immobilised homogeneous catalysis}

The most common approach for homogeneous catalyst recycling is the immobilisation onto solid supports. The immobilisation of homogeneous complexes on a solid support renders a system that can be easily separated from the reaction media and reused in subsequent runs. One of the drawbacks of this strategy is that the immobilisation of homogeneous catalysts usually affords less active systems due to mass transfer problems and the added difficulty to access the active sites.

In this context, there are two main methodologies to immobilise a metal complex onto a solid support via either covalent or non-covalent interactions (Scheme 1.1). The most widely used implies the formation of a covalent bond between the molecular catalyst and the support. Thus, the insertion of suitable functional groups in at least the catalyst or the support is needed, which adds extra synthetic steps. In addition, the structural modification of the catalyst may alter its performance, leading to less active catalysts. Following this strategy, Prof. P. Van Leeuwen and co-workers described in 1998 a recyclable rhodium catalyst immobilised on silica. ${ }^{[9,10]}$ The catalyst is based on a xanthene scaffold that is modified to introduce a siloxane moiety. The condensation of the siloxane fragment with tetramethylorthosilicate generates a Rh-supported catalyst (Figure 1.4). This new platform efficiently catalyses the hydroformylation of 1-octene with high selectivity towards the more valuable linear product. In addition, the catalyst can be separated from the reaction mixture by easy filtration and reused. Consistent yields and selectivities were 
obtained through eight runs, thus demonstrating the robustness of the catalyst. Besides, this system is one of the first heterogenised catalysts lacking metal leaching (less than $1 \%$ after each run).

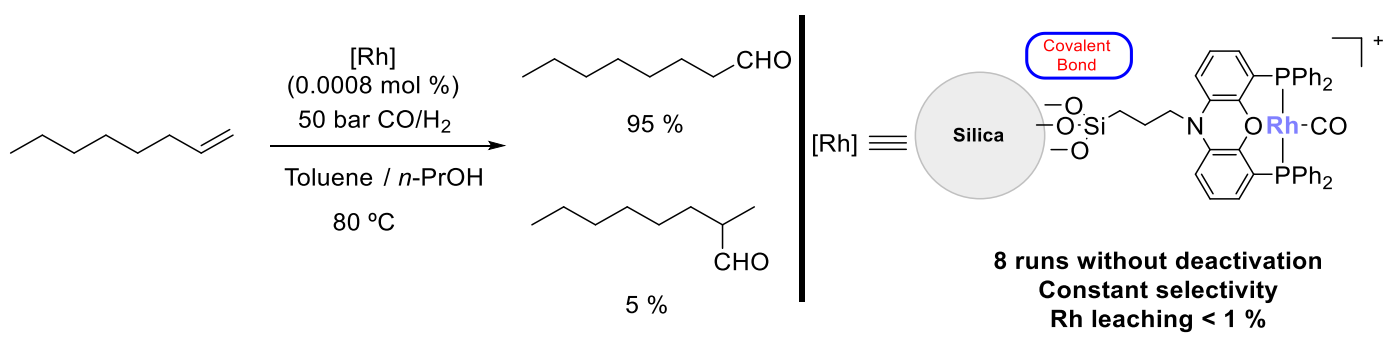

Figure 1.4 Hydroformylation of 1-octene catalysed by silica-supported Rh catalyst

As an alternative to the covalent-grafting, non-covalent interactions are emerging as a powerful tool to immobilise homogeneous catalysts on solid supports. The approach based on non-covalent interactions has the advantage that pre-functionalisation of the catalyst or the support may not be necessary. This is the case where molecular compounds are immobilised by simple physisorption. ${ }^{[11-13]}$ However, in order to avoid significant metal leaching, a careful catalyst design is required in some cases to ensure a strong enough interaction between the catalyst and the support. The implementation of this strategy is straightforward (materials are prepared by simple mixing in a solvent) and resembles the impregnation method used for the synthesis of nanoparticles on solid supports. ${ }^{[14]}$ Following this method, Balcar and co-workers reported a silica-immobilised ruthenium catalyst active in olefin metathesis (Figure 1.5). ${ }^{[15]}$

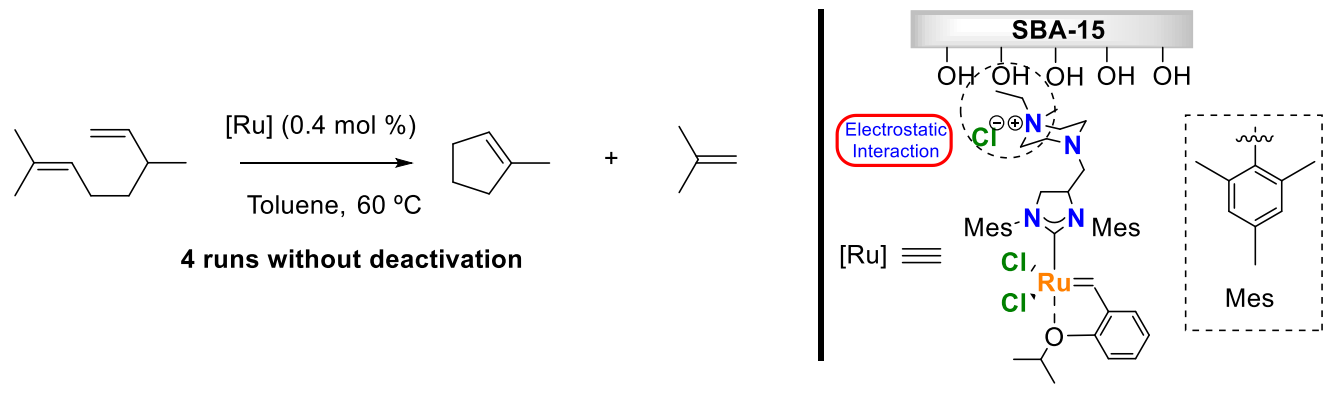

Figure 1.5 Metathesis of olefins catalysed by a $2^{\text {nd }}$ generation-modified Hoveyda-Grubbs catalyst immobilised onto silica by electrostatic interactions 
Starting from the commercially available complex with a quaternary ammonium group, the authors prepared the solid version of the catalyst anchoring the molecular complex on different silica-based materials. The driving forces that maintained the catalyst on the silica are the electrostatic interactions between the ammonium tag and the silanol groups located at the surface of silica. Among the different tested materials, SBA-15 with a hierarchical porosity and a large pore diameter gave the best results. The catalyst was active for four consecutive runs without decrease in activity. In order to elucidate the nature of the active catalytic species, the hot filtration test, which is often used in supported catalysis, was carried out. ${ }^{[16]}$ It consists on the filtration "in hot" of the reaction mixture when a ca. $50 \%$ conversion is reached. Then, the solid catalyst is treated with more solvent and substrate and heated again at the reaction temperature. In parallel, the filtrate is left to react under the reaction conditions. Two possible scenarios can be found:

a) The reaction in the filtrate does not proceed. In this case the heterogeneous nature of the catalyst is demonstrated, as no leached species are present in the filtrate. The result is accompanied by the maintenance of the activity of the solid catalyst. This was the situation found with the system depicted in Figure 1.5.

b) The reaction in the filtrate proceeds. This indicates that the molecular complex is desorbed from the surface during the reaction. In this situation the catalytic activity may be caused by both, the leached species and the heterogeneous supported catalyst. The activity of the separated solid catalyst determines the nature of the active species. If the immobilised catalyst is completely inactive, the catalyst performance is attributed only to the leached species. On the contrary, if the solid retains some activity, the transformation of substrates into products is mediated by both, the heterogeneous catalyst and the leached species.

In some cases, when the hot filtration test gives a positive result (i.e. confirms the presence of active species in solution) the catalyst can still be recycled. This is called "boomerang effect" or "the catch and release mechanism". ${ }^{[17]}$ The boomerang effect occurs when during the reaction the molecular complex grafted on a solid support is released and does the catalysis in the homogeneous phase. Then, when the reaction is finished, the homogeneous complex can be re-immobilised on the support before the separation of the catalyst from the reaction media. This situation is most commonly observed when using the non-covalent approach where the "weak" 
non-covalent interactions are non-existent or very feeble at the reaction temperature. Then, when the reaction is cooled down the interactions are re-established and the homogeneous catalysts are re-anchored on the solid matrix (Figure 1.6).

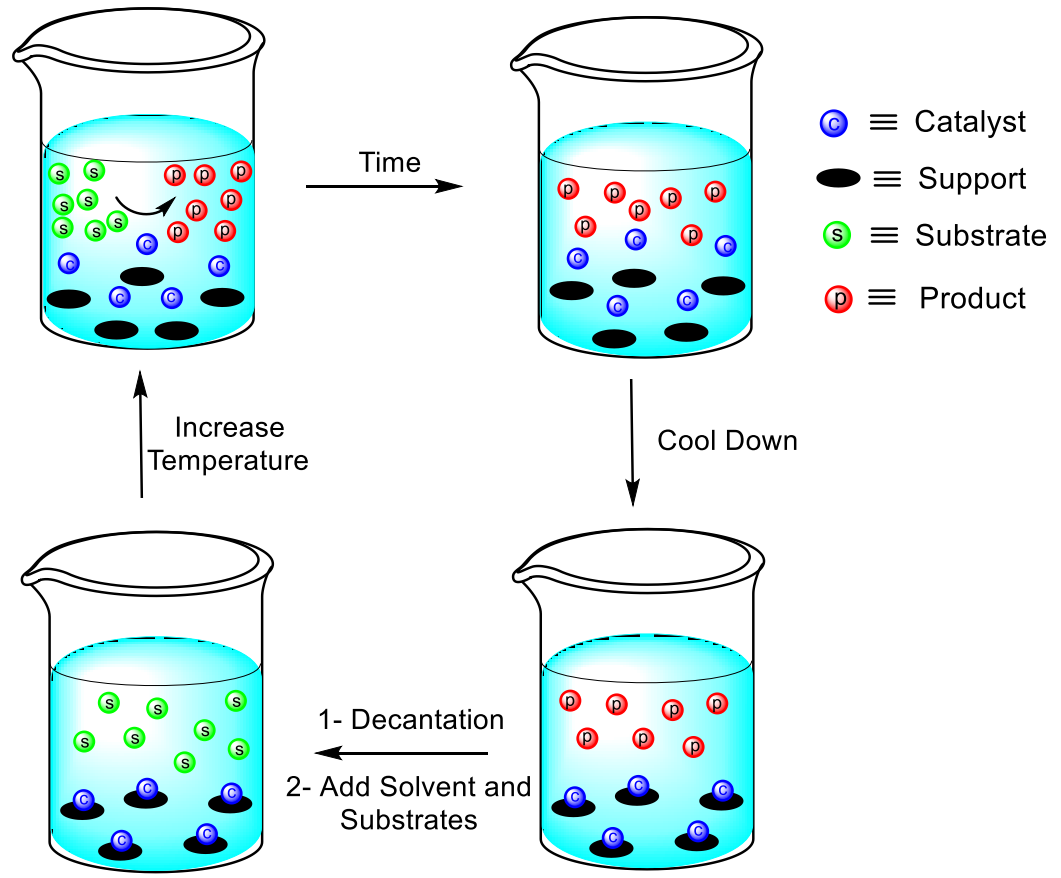

Figure 1.6 Schematic representation of the boomerang effect

\subsection{CHEMICALLY DERIVED GRAPHENES AS SUPPORTS}

Traditionally, the heterogeneisation of transition metal complexes has been carried out using silica or polymeric supports. ${ }^{[18]}$ However, carbon materials offer advantages over these supports such as increased mechanical and thermal stability, high surface area and an especially rich surface chemistry. That is why in the last years there is an increasing interest in the use of carbon materials as supports. ${ }^{[19,20]}$ In particular, graphene and chemically derived graphenes (CDGs) have drawn a lot of attention.

Graphene is a two-dimensional layer of one atom thick $\mathrm{sp}^{2}$ carbons in an hexagonal arrangement. It was discovered in 2004 by A. Geim and K. Novoselov, ${ }^{[21]}$ who were awarded the Nobel Prize in physics in 2010. The excellent optical, electrical and mechanical properties of graphene have attracted the interest of all the scientific community. Pure graphene is an unreactive and very expensive material so its use as 
support for the immobilisation of catalysts is not viable. The difficulties found in the synthesis of pure graphene have prompted the interest on derived graphene nanocomposites and hybrids for real applications. Chemically derived graphenes such as graphene oxide (GO) and reduced graphene oxide ( $\mathrm{rGO}$ ), are ideal candidates for the immobilisation of active transition metal complexes for catalytic applications due to its inertness, high surface area, stability, large scale availability and presence of functional groups.

\subsubsection{Preparation of chemically derived graphenes}

Graphene derivatives are complex materials considering the composition, number and nature of functional groups and the size and number of sheets. The control of all these factors depends on the methodology used for their synthesis and determines the inherent properties of the material. ${ }^{[22]}$ CDGs can be prepared following different strategies, being the chemical procedures the most common. The preparation of chemically derived graphenes is based on a sequence of redox reactions and exfoliation processes (Figure 1.7).

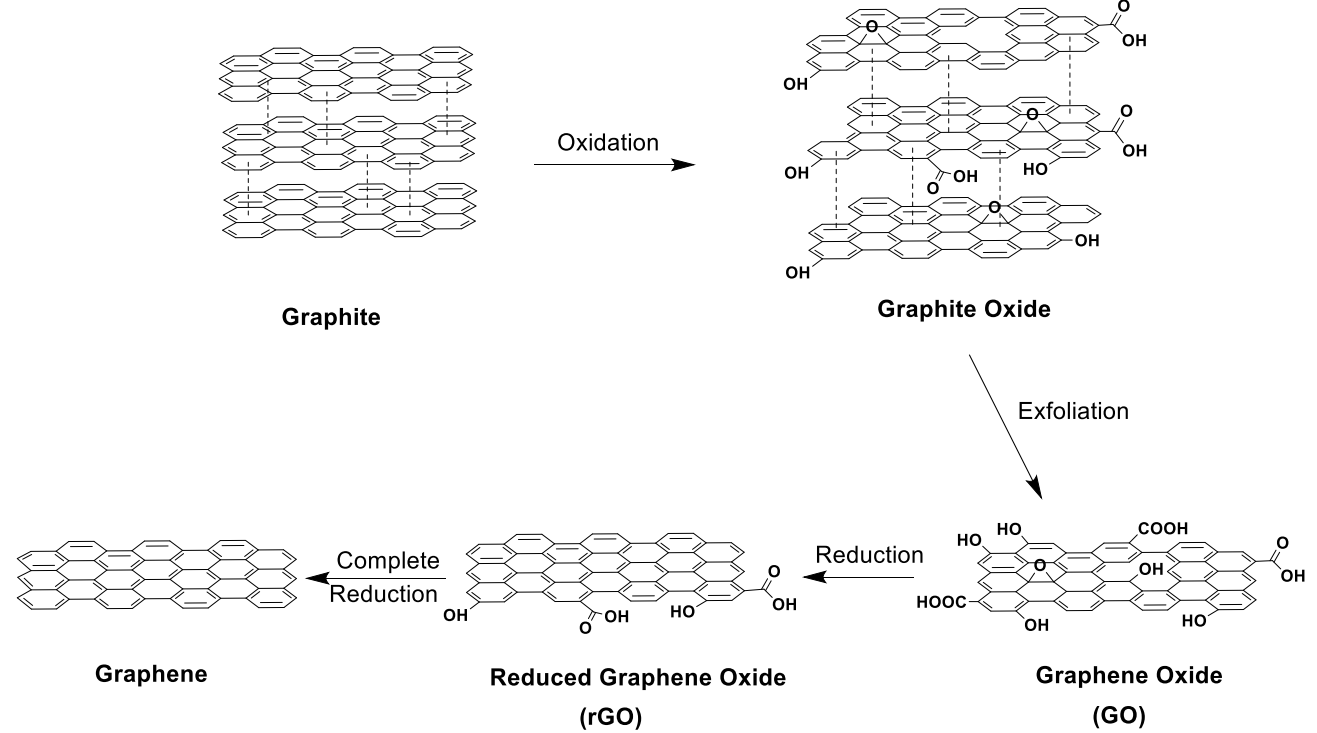

Figure 1.7 Synthesis of chemically derived graphenes

The oxidation of graphite with strong oxidants forms graphite oxide, a 3D material that contains functional groups as epoxides, hydroxyls and carboxylic acids. Graphite oxide was first developed by B.C. Brodie in 1859, using nitric acid and potassium chlorate, ${ }^{[23]}$ but today the most common method for the synthesis of graphite oxide is the Hummers method, using potassium permanganate and sulfuric acid. ${ }^{[24]}$ 
The introduction of oxygen functionalities increases the distance between layers, and reduces the strength of $\pi-\pi$ interactions. Consequently, graphite oxide is easily exfoliated by ultrasounds, yielding graphene oxide (GO). Graphene oxide is a carbon-based 2D material with several oxygenated functional groups at the basal planes (hydroxyls and epoxides) and at the edges (hydroxyls and carboxylic acids). The presence of these functional groups disrupts the electronic structure of pristine graphene, leading to an insulating material. The next step is the reduction of GO, yielding reduced graphene oxide ( $\mathrm{rGO}$ ). The complete reduction of $\mathrm{GO}$ should give pristine graphene, but this reaction is very difficult and in practice only $\mathrm{rGO}$ is obtained. The most common procedure for obtaining rGO is the chemical reduction with hydrazine, ${ }^{[25]}$ although other alternatives have been reported. ${ }^{[2,27]}$ The properties of rGO are between pristine graphene and graphene oxide. For example, its surface area is about $450 \mathrm{~m}^{2} / \mathrm{g}$, which is considerably higher than the surface area of GO $\left(20-30 \mathrm{~m}^{2} / \mathrm{g}\right)$ but far from the surface area of pristine graphene (estimated to be $2600 \mathrm{~m}^{2} / \mathrm{g}$ ). ${ }^{[28]}$ Interestingly, the reduction process of $\mathrm{GO}$ eliminates some oxygen functionalities, so the $\pi$ network of the material is restored, making $r G O$ a conductive material.

\subsubsection{Covalent and non-covalent immobilisation of molecular complexes on chemically derived graphenes}

The use of CDGs as supports for catalytic applications has drawn a considerable attention. Both, the covalent and the non-covalent approach have been studied for the grafting of molecular complexes on CDGs.

The rich (yet complex) surface chemistry of CDGs allows the insertion of specific functionalities that can serve as platforms for the formation of a covalent bond between the support and the catalyst. For the covalent approach, GO is more widely used due to the presence of several oxygenated functional groups that can be modified. Although the presence of the oxygenated functionalities can be regulated in a certain degree, ${ }^{[19]}$ the main drawback of using GO as support is the lack of control of the precise location of the catalytic active centres on the support. As consequence, the surface chemistry of GO governs the catalyst dispersion, which is not always homogeneous.

Hydroxyls, epoxides and carboxylic acids are the functionalities usually present at the surface of GO, although other functionalities such as lactones or quinones may be present depending on the synthetic procedure used. In general, surface modification strategies exploit phenol groups because these are present at the basal 
plane and at the edges of GO, thus ensuring a homogeneous distribution. ${ }^{[19,29-31]}$ Nonetheless, the immobilisation of complexes through carboxylic acid functionalities has also been reported. ${ }^{[32,33]}$

Silylation is the most common strategy used for the immobilisation of complexes onto graphene oxide. ${ }^{[29-31,34-39]}$ In analogy to silica materials, the superficial hydroxyl groups of GO can react with the tri-alkoxysilane functionality contained in a ligand or a complex. As a result, the final catalyst is strongly anchored to the surface of GO through a siloxane-type bond. Using this approach, the widely used catalyst $\left[\mathrm{Ru}\left(\mathrm{PPh}_{3}\right)_{3} \mathrm{Cl}_{2}\right]$ was grafted onto $\mathrm{GO}$ and the catalytic properties of the new hybrid material were studied (Figure 1.8). ${ }^{[29]}$ Reaction of graphene oxide with 3-(aminopropyl)trimethoxysilane affords the amino functionalised $\mathrm{GO}$ with $\mathrm{NH}_{2}$ pendant groups $\left(\mathrm{NH}_{2}-\mathrm{GO}\right)$. Treatment of $\mathrm{NH}_{2}-\mathrm{GO}$ with $\left[\mathrm{Ru}\left(\mathrm{PPh}_{3}\right)_{3} \mathrm{Cl}_{2}\right]$ produces the exchange of one triphenylphosphine for one of the amino ligands of the material, rendering the immobilisation of the Ru complex on the solid support.

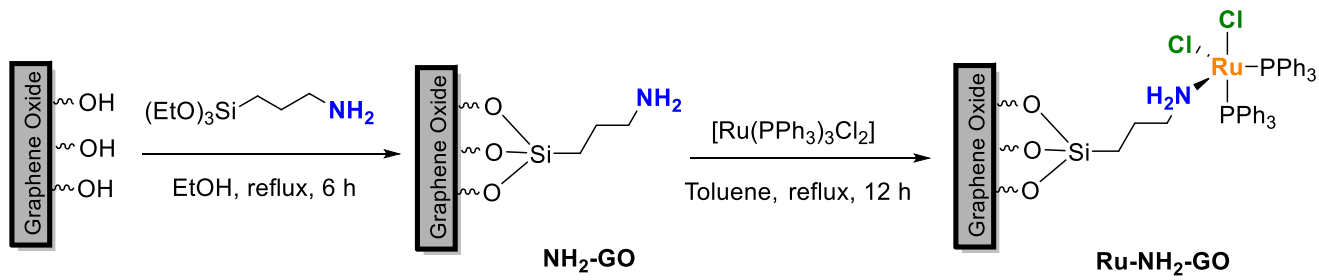

Ru-NH $\mathbf{N}_{\mathbf{2}}$-GO

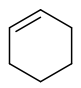

$(0.1 \mathrm{~mol} \%)$

10 bar $\mathrm{H}_{2}$, toluene $70^{\circ} \mathrm{C}$
5 runs without deactivation

\begin{tabular}{|c|c|c|c|c|c|}
\hline Run & 1 & 2 & 3 & 4 & 5 \\
\hline Yield $(\%)$ & 98 & 97 & 96 & 95 & 95 \\
\hline TOF $\left(\mathrm{s}^{-1}\right)$ & 500 & 500 & 495 & 495 & 490 \\
\hline
\end{tabular}

Increased activity respect to the molecular catalyst Negligible Ru leaching

Figure 1.8 Covalent immobilisation of a Ru complex on GO and catalytic properties

The new catalyst was tested in the reduction of alkenes and ketones. The results showed that the new platform has a good activity in the reduction of alkenes and a moderate activity for the reduction of ketones. Interestingly, competitive experiments revealed a high selectivity towards the reduction of olefins, which were fully converted to the corresponding alkanes leaving the ketone functionality almost unaltered. The solid-supported catalyst was very robust and five consecutive runs could be performed without a discernible loss in activity. Analyses by Inductively 
Coupled Plasma-Mass Spectrometry (ICP-MS) after the recycling experiments were consistent with the negligible deactivations and showed a low metal contamination of the final products. For comparative purposes, $\left[\mathrm{Ru}\left(\mathrm{PPh}_{3}\right)_{3} \mathrm{Cl}_{2}\right]$ was also examined under the same reaction conditions. The results reveal an enhanced performance of the supported catalyst. This observation is not common for heterogeneised catalysts, where usually the heterogeneous material shows lower activity than its homogeneous counterpart due to mass transfer problems. When using GO, the flat 2D nature of the material allows the easy access of reactants to the catalytically active centres, which are not "hindered" as it may happen in silica materials with a hierarchical porosity. That is why, with a sufficiently intense stirring, mass transfer problems are avoided. According to the authors, the enhanced catalytic performance can also be attributed to the site isolation effect. ${ }^{[40]}$ Under homogeneous conditions, $\left[\mathrm{Ru}\left(\mathrm{PPh}_{3}\right)_{3} \mathrm{Cl}_{2}\right.$ ] can form dimmers and aggregates, which are inactive in catalysis. When the molecular complex is grafted onto rGO, the support keeps the molecules of the catalyst far enough to prevent dimerization reactions, thus increasing the stability of the catalyst.

Another effect that may help to the increase in the catalytic activity of $\mathbf{R u}-\mathbf{N H}_{\mathbf{2}}-\mathbf{r G O}$ is the modification of the structure of the molecular catalyst after the immobilisation process. The replacement of one $\mathrm{PPh}_{3}$ by one amino ligand can also affect the catalytic behaviour by increasing the electron density of the Ru centre. However, the exact coordination sphere around the metal is not completely known.

As an alternative to the formation of a covalent bond between the catalyst and the carbonaceous surface, non-covalent interactions have also been studied. Non-covalent interactions include electrostatic interactions, $\pi-\pi$ stacking, adsorption and supramolecular interactions. The extended $\pi$-cloud of reduced graphene oxide along with its high surface area make rGO an excellent candidate to anchor molecular complexes by $\pi-\pi$ interactions. The $\pi-\pi$ stacking interactions are Van der Waals forces in nature, and are controlled by thermodynamics. The energy of a single $\pi$-stacking interaction is low (about $2 \mathrm{~kJ} / \mathrm{mol}$ ), ${ }^{[41]}$ but the energy for the whole interaction between a polyaromatic system and $\mathrm{rGO}$ depends on the number of aromatic rings and on the contact curvature between the aromatic region of the molecule and the surface of graphene materials.

The $\pi-\pi$ interaction occurs when a polyaromatic system contacts the graphitic $\mathrm{sp}^{2}$ carbon network of graphene. The immobilisation is commonly carried out by simple solution mixing of the support and the molecular catalyst in a solvent. The process is 
straightforward and no modification of the support is required. On the contrary, this approach always needs the introduction of a polyaromatic group (usually a pyrene) in the coordination sphere of the molecular compound. These modifications in the ligands are usually carried out in one to three synthetic steps, starting from commercially available compounds and using well-known organic reactions. This methodology has the advantage that the catalyst is pre-formed prior to its immobilisation. In this way the structure of the molecular compound when it is grafted on graphene is well-defined.

Immobilisation of organometallic complexes by $\pi-\pi$ interactions is well documented using carbon nanotubes as support. Carbon nanotubes are graphene sheets rolled into a cylindrical shape. The cylinders have a high $\pi$-electron density on the surface and thus can serve to anchor molecular compounds through non-covalent interactions. Catalytically active materials have been obtained by grafting complexes of ruthenium, ${ }^{[42,43]}$ palladium, ${ }^{[44]}$ iron, ${ }^{[45]}$ rhodium ${ }^{[46]}$ or gold. ${ }^{[47]}$ However, the use of rGO as support for catalytic studies remained almost unstudied at the beginning of the present PhD thesis.

\subsubsection{Precedents in our research group}

Our research group has a strong background in the synthesis of new organometallic compounds and the study of their catalytic properties. In particular, the group has centred most of its efforts in the study of metal complexes with $\mathrm{N}$-heterocyclic carbene ligands (NHCs).

Carbenes are organic species that feature a divalent carbon with six valence electrons. The incomplete electron shell renders free carbenes as very unstable species. The interest in the isolation of free carbenes dates back to 1835, when J.P. Dumas tried to isolate methylene (the simplest carbene), by dehydration of methanol using phosphorous pentoxide. ${ }^{[48]}$ However, it was not until 1988 when G. Bertrand and co-workers reported the first isolable carbene stabilised by adjacent silicon and phosphorous atoms. ${ }^{[49]}$ Three years later, A. Arduengo reported and isolated the first free $\mathrm{N}$-heterocyclic carbene, ${ }^{[50]}$ which caused a real explosion in the studies of the coordination chemistry of NHCs. ${ }^{[51]}$ Carbenes can coordinate to a metal forming strong metal-carbon (M-C) bonds. The success in the use of carbenes as ligands in organometallic chemistry relies on its strong $\sigma$-donating capacity, ${ }^{[52]}$ which allows in most cases the formation of strong $\mathrm{M}-\mathrm{C}$ bonds and prevents decomposition of the catalyst. ${ }^{[53]}$ 
By definition, NHCs are cyclic carbenes bearing at least one $\alpha$-amino substituent. Imidazolylidenes are among the most studied carbenes in coordination chemistry. The fine-tuning of the electronic and steric properties of the ligand, along with the possibility of the functionalisation through the backbone, make imidazolylidenes powerful ligands with numerous applications in catalysis (Figure 1.9). ${ }^{[54]}$

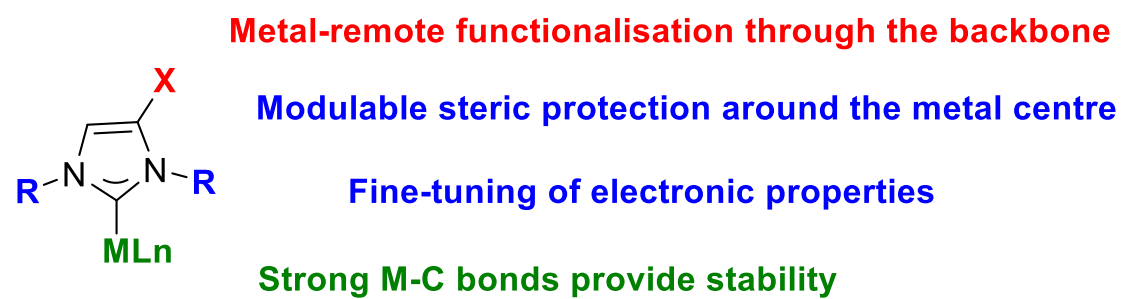

Figure 1.9 Fine tuning of electronic properties and steric control of imidazolylidines

Thanks to the versatility of imidazolylidenes, Sabater, Mata and Peris reported in 2014 a pyrene-tagged imidazolium salt that served as ligand precursor for the synthesis of NHC-complexes of ruthenium and palladium. The presence of the pyrene tag allowed the immobilisation of the complexes onto $\mathrm{rGO}$ by $\pi$-stacking interactions, rendering the new hybrid materials Ru-NHC-rGO and Pd-NHC-rGO (Figure 1.10). ${ }^{[55]}$

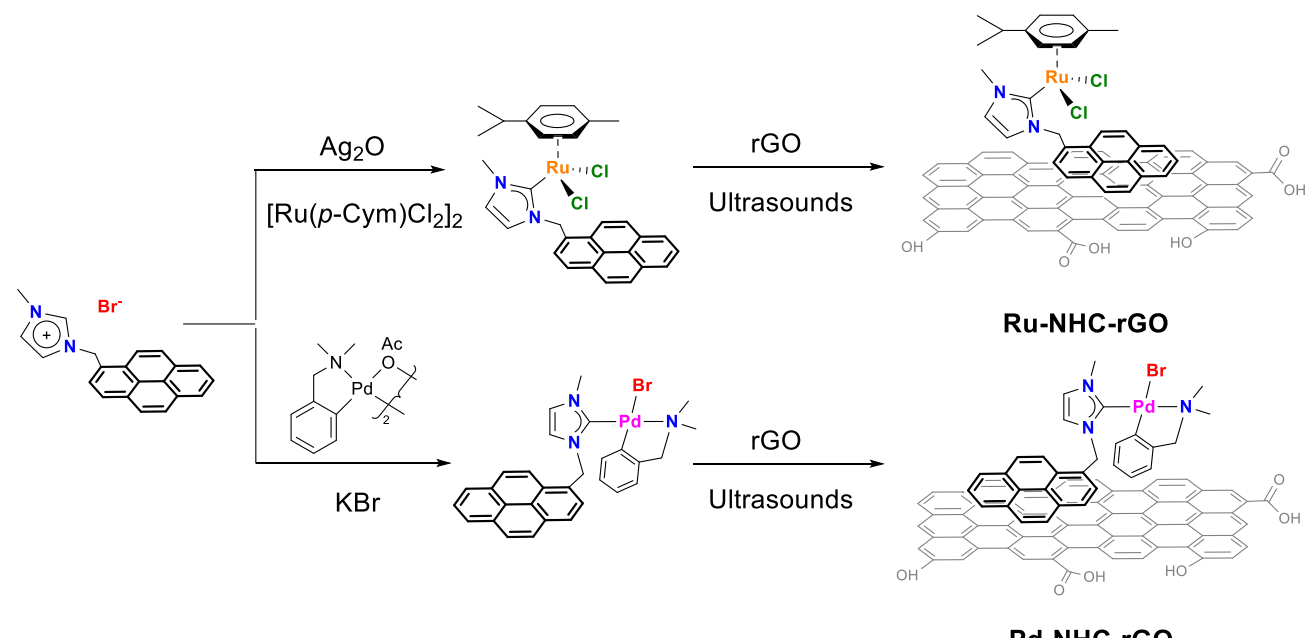

Figure 1.10 Synthesis of Ru-NHC-rGO and Pd-NHC-rGO hybrid materials 
The catalytic properties of Pd-NHC-rGO and Ru-NHC-rGO were tested in reactions typically catalysed by these metals. The Pd-NHC complex is very active in the reduction of alkenes and nitroarenes using molecular hydrogen at one bar of pressure. Interestingly, the yields obtained using the molecular complex of Pd are improved when Pd-NHC-rGO is used as catalyst. The recyclability studies showed that the Pd hybrid material could be reused up to 10 times without apparent loss of activity. The time course recycling experiment using low catalyst loadings (0.01 mol \%) reveals that the catalyst activity is maintained at different times for three runs. The palladium hybrid material was analyzed after 10 runs by high resolution transmission electron microscopy (HRTEM) and inductively coupled plasma mass spectrometry (ICP-MS). The ICP-MS analysis reveals that the palladium leaching is less than $10 \%$. Images taken by HRTEM show the formation of well-dispersed nanoparticles, indicating that the molecular catalyst is decomposed during the catalytic reaction rendering highly active palladium nanoparticles anchored onto rGO.

The ruthenium hybrid material was tested in the oxidation of primary and secondary alcohols to the corresponding aldehydes and ketones. The results show that the molecular complex is less active than the Ru-NHC-rGO material, indicating that the immobilisation of the molecular complex onto rGO improves the catalytic properties. This material was reused up to ten times without apparent deactivation of the catalyst, and the catalyst activity is maintained at different reaction times as shown by the time course recycling experiment (Figure 1.11). 

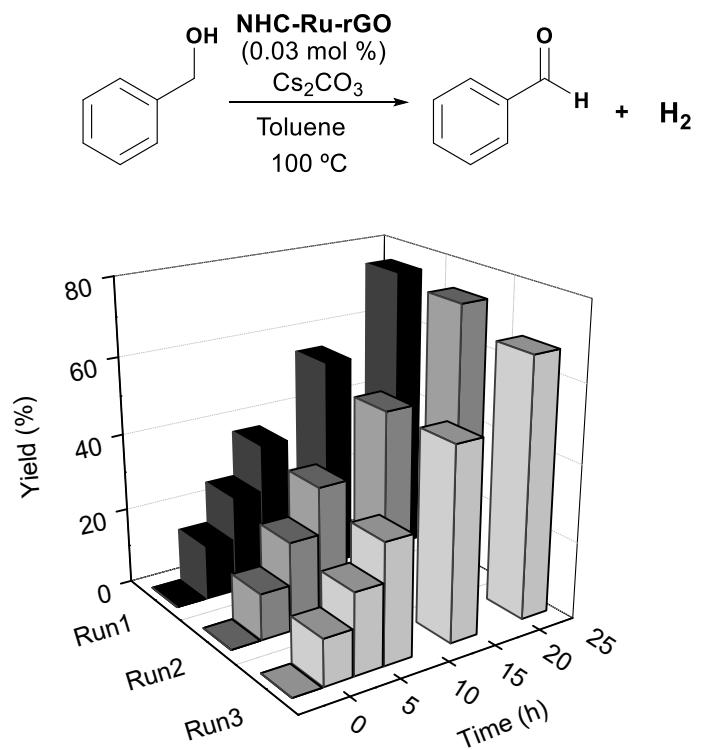

Figure 1.11 Time course recycling experiment for the benzyl alcohol oxidation using Ru-NHC-rGO at a catalyst loading of $0.03 \mathrm{~mol} \%$

The analysis by ICP-MS of the ruthenium hybrid material after the recycling experiments shows that the amount of $\mathrm{Ru}$ desorbed from $\mathrm{rGO}$ is negligible. Morphological analysis carried out by TEM reveal a well-dispersed ruthenium distribution without modification of support morphology, and the absence of ruthenium nanoparticles. This result confirms the molecular nature of the metallic species attached to the support, and provides the opportunity of controlling the electronic and steric properties of the catalytic active site by modifying the ligands of the molecular complex. 


\subsection{OBJECTIVES}

After the first publication of the new strategy to graft molecular complexes onto graphene, our group turned its attention to the study of recyclable homogeneous catalysts. Based on these precedents, the general objective of this PhD. Thesis is to fully exploit and understand the properties of the new hybrid materials. This general objective can be divided in the following:

- Design and synthesis of new imidazolium salts with polyaromatic tags that would serve as ligand precursors for the synthesis of M-NHC complexes.

- Coordination of NHC ligands to different metal fragments.

- Immobilisation of the new complexes onto reduced graphene oxide and full characterisation to determine the nature of the immobilised species.

- Study of the catalytic properties of the new hybrid materials, paying special attention to the recycling properties.

- Characterisation of the materials after the catalysis to determine the mechanism that governs the deactivation of the catalysts, if any.

- Study of the reaction mechanisms of the catalytic reactions. 


\subsection{REFERENCES}

[1] J. G. De Vries, S. D. Jackson, Catal. Sci. Technol. 2012, 2, 2009.

[2] S. L. Wegener, T. J. Marks, P. C. Stair, Acc. Chem. Res. 2012, 45, 206-214.

[3] Y. Yang, N. Priyadarshani, T. Khamatnurova, J. Suriboot, D. E. Bergbreiter, J. Am. Chem. Soc. 2012, 134, 14714-14717.

[4] I. T. Horváth, G. Kiss, R. A. Cook, J. E. Bond, P. A. Stevens, J. Rábai, E. J. Mozeleski, J. Am. Chem. Soc. 1998, 120, 3133-3143.

[5] H. D. Velazquez, F. Verpoort, Chem. Soc. Rev. 2012, 41, 7032-7060.

[6] G. J. Ten Brink, I. W. C. E. Arends, R. A. Sheldon, Science 2000, 287, 16361639.

[7] R. Kawahara, K. Fujita, R. Yamaguchi, J. Am. Chem. Soc. 2012, 134, 36433646.

[8] J. Campos, Phys. Sci. Rev. 2018, 3, 1-25.

[9] A. J. Sandee, L. A. van der Veen, J. N. H. Reek, P. C. J. Kamer, M. Lutz, A. L. Spek, P. W. N. M. van Leeuwen, Angew. Chemie - Int. Ed. 1999, 38, 32313235.

[10] A. J. Sandee, J. N. H. Reek, P. C. J. Kamer, P. W. N. M. Van Leeuwen, J. Am. Chem. Soc. 2001, 123, 8468-8476.

[11] S. A. C. Carabineiro, L. M. D. R. S. Martins, A. J. L. Pombeiro, J. L. Figueiredo, ChemCatChem 2018, 10, 1804-1813.

[12] J. Cabrera, R. Padilla, M. Bru, R. Lindner, T. Kageyama, K. Wilckens, S. L. Balof, H. J. Schanz, R. Dehn, J. H. Teles, et al., Chem. - A Eur. J. 2012, 18, 1471714724.

[13] E. Choi, C. Lee, Y. Na, S. Chang, Org. Lett. 2002, 4, 2369-2371.

[14] A. Corma, H. Garcia, Chem. Soc. Rev. 2008, 37, 2096-2126.

[15] J. Pastva, K. Skowerski, S. J. Czarnocki, N. Žilková, J. Čejka, Z. Bastl, H. Balcar, ACS Catal. 2014, 4, 3227-3236.

[16] J. E. Hamlin, K. Hirai, V. C. Gibson, P. M. Maitlis, J. Mol. Catal. 1982, 15, 337347.

[17] M. Gruttadauria, F. Giacalone, R. Noto, Green Chem. 2013, 15, 2608-2618. 
[18] W. Wang, L. Cui, P. Sun, L. Shi, C. Yue, F. Li, Chem. Rev. 2018, 118, 9843-9929.

[19] M. R. Axet, O. Dechy-Cabaret, J. Durand, M. Gouygou, P. Serp, Coord. Chem. Rev. 2016, 308, 236-345.

[20] M. R. Axet, J. Durand, M. Gouygou, P. Serp, Adv. Organomet. Chem. 2019, 71, 53-174.

[21] K. S. Novoselov, A. K. Geim, S. V Morozov, D. Jiang, Y. Zhang, S. V. Dubonos, I. V Grigorieva, A. A. Firsov, Science 2004, 306, 666-669.

[22] A. Ambrosi, C. K. Chua, A. Bonanni, M. Pumera, Chem. Rev. 2014, 114, 71507188.

[23] B. C. Brodie, Philos. Trans. R. Soc. London 1859, 149, 249-259.

[24] W. S. Hummers, R. E. Offeman, J. Am. Chem. Soc. 1958, 80, 1339.

[25] S. Stankovich, D. A. Dikin, R. D. Piner, K. A. Kohlhaas, A. Kleinhammes, Y. Jia, Y. Wu, S. B. T. Nguyen, R. S. Ruoff, Carbon 2007, 45, 1558-1565.

[26] N. A. Kumar, S. Gambarelli, F. Duclairoir, G. Bidan, L. Dubois, J. Mater. Chem. A 2013, 1, 2789-2794.

[27] S. Abdolhosseinzadeh, H. Asgharzadeh, H. S. Kim, Sci. Rep. 2015, 5, 10160.

[28] S. Sabater, J. A. Mata, in Non-Covalent Interactions in the Synthesis and Design of New Compounds, Jhon Wiley \& Sons, Inc, 2016, pp. 315-326.

[29] Q. Zhao, Y. Li, R. Liu, A. Chen, G. Zhang, F. Zhang, X. Fan, J. Mater. Chem. A 2013, 1, 15039-15045.

[30] Q. Zhao, C. Bai, W. Zhang, Y. Li, G. Zhang, F. Zhang, X. Fan, Ind. Eng. Chem. Res. 2014, 53, 4232-4238.

[31] C. Bai, Q. Zhao, Y. Li, G. Zhang, F. Zhang, X. Fan, Catal. Letters 2014, 144, 1617-1623.

[32] A. Hassanpour, D. Rodriguez-San Miguel, J. L. G. Fierro, B. R. Horrocks, R. Mas-Ballesté, F. Zamora, Chem. - A Eur. J. 2013, 19, 10463-10467.

[33] M. B. Avinash, K. S. Subrahmanyam, Y. Sundarayya, T. Govindaraju, Nanoscale 2010, 2, 1762-1766.

[34] A. B. Dongil, B. Bachiller-Baeza, A. Guerrero-Ruiz, I. Rodríguez-Ramos, J. Catal. 2011, 282, 299-309. 
[35] S. Verma, M. Aila, S. Kaul, S. L. Jain, RSC Adv. 2014, 4, 30598-30604.

[36] H. P. Mungse, S. Verma, N. Kumar, B. Sain, O. P. Khatri, J. Mater. Chem. 2012, 22, 5427-5433.

[37] A. B. Dongil, B. Bachiller-Baeza, A. Guerrero-Ruiz, I. Rodríguez-Ramos, Catal. Commun. 2012, 26, 149-154.

[38] F. Rao, S. Deng, C. Chen, N. Zhang, Catal. Commun. 2014, 46, 1-5.

[39] M. A. Nasseri, A. Allahresani, H. Raissi, RSC Adv. 2014, 4, 26087-26093.

[40] R. H. Grubbs, C. Gibbons, L. R. Kroll, W. D. Bonds, C. H. Brubaker, J. Am. Chem. Soc. 1973, 95, 2373-2375.

[41] C. Janiak, J. Chem. Soc. Dalton Trans. 2000, 3885-3896.

[42] F. J. Gómez, R. J. Chen, D. Wang, R. M. Waymouth, H. Dai, Chem. Commun. 2003, 9, 190-191.

[43] G. Liu, B. Wu, J. Zhang, X. Wang, M. Shao, J. Wang, Inorg. Chem. 2009, 48, 2383-2390.

[44] Y. Suzuki, P. Laurino, D. T. Mcquade, P. H. Seeberger, Helv. Chim. Acta 2012, 95, 2578-2588.

[45] L. Zhang, W. Zhang, P. Serp, W. H. Sun, J. Durand, ChemCatChem 2014, 6, 1310-1316.

[46] L. Xing, J. Xie, Y. Chen, L. Wang, Q. Zhou, Adv. Synth. Catal. 2008, 350, 10131016.

[47] C. Vriamont, M. Devillers, O. Riant, S. Hermans, Chem. - A Eur. J. 2013, 19, 12009-12017.

[48] J. B. Dumas, E. Peligot, Ann. Chim. Phys. 1835, 58, 5.

[49] A. Igau, H. Grutzmacher, A. Baceiredo, G. Bertrand, J. Am. Chem. Soc. 1988, $110,6463-6466$.

[50] A. J. Arduengo, R. L. Harlow, M. Kline, J. Am. Chem. Soc. 1991, 113, 361-363.

[51] S. Díez-González, N. Marion, S. P. Nolan, Chem. Rev. 2009, 109, 3612-3676.

[52] S. Díez-González, S. P. Nolan, Coord. Chem. Rev. 2007, 251, 874-883. 
[53] H. Jacobsen, A. Correa, A. Poater, C. Costabile, L. Cavallo, Coord. Chem. Rev. 2009, 253, 687-703.

[54] E. Peris, Chem. Rev. 2018, 118, 9988-10031.

[55] S. Sabater, J. A. Mata, E. Peris, ACS Catal. 2014, 4, 2038-2047. 



\section{CHAPTER 2}

Ru-NHC-rGO as Reusable Catalysts for the Dehydrogenation of Alcohols in Aqueous Media

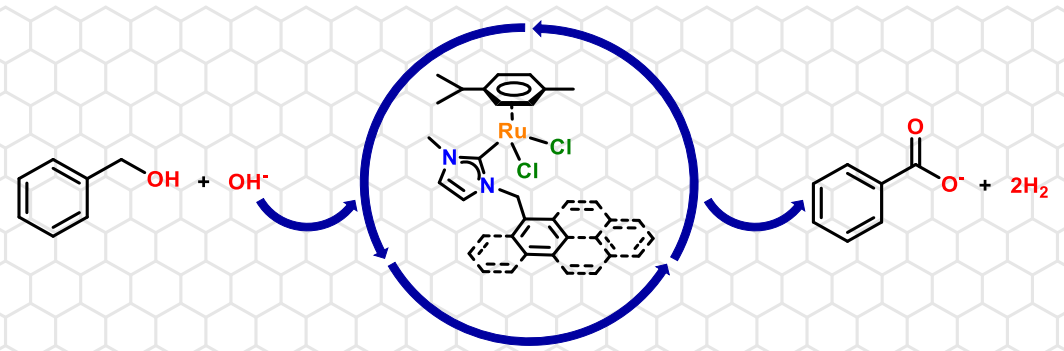





\subsection{INTRODUCTION}

The selective oxidation of primary alcohols to carboxylic acids is a challenging transformation that has drawn the attention of both, academy and industry. Traditionally, alcohol oxidation is carried out using strong oxidants such as potassium permanganate $\left(\mathrm{KMnO}_{4}\right)$ or potassium dichromate $\left(\mathrm{K}_{2} \mathrm{Cr}_{2} \mathrm{O}_{7}\right) \cdot{ }^{[1,2]}$ These methods suffer from a poor atom economy and large amounts of metal wastes are generated, which make them less attractive for large scale production (Figure 2.1). ${ }^{[3]}$

$$
\mathrm{R} \widehat{\mathrm{OH}} \stackrel{\text { Metal salts }}{\longrightarrow} \mathrm{R}_{\mathrm{OH}}^{\text {省 }}+\begin{gathered}
\text { Stoichiometric amount } \\
\text { of waste }
\end{gathered}
$$

Figure 2.1 Classic synthesis of carboxylic acids

As alternative, metal catalysed procedures have arisen as a powerful tool towards more efficient methods. It is worth mentioning that, while the oxidation of primary and secondary alcohols to aldehydes and ketones using homogeneous and heterogeneous catalysts has been intensively studied, ${ }^{[4]}$ the direct oxidation of primary alcohols to carboxylic acids has been less explored. However, some homogeneous and heterogeneous systems have been reported to efficiently catalyse the selective synthesis of carboxylic acids from primary alcohols. ${ }^{[4-6]}$ Depending on the terminal oxidant, these catalytic processes can be divided in: i) catalytic systems where oxygen is the terminal oxidant (A) in Figure 2.2) ii) systems in which a catalyst dehydrogenates an alcohol in the presence of a hydrogen acceptor (B) in Figure 2.2) iii) systems where the catalyst dehydrogenates the alcohol in the absence of a hydrogen acceptor (acceptorless dehydrogenation (ADH), C) in Figure 2.2) 
A) $\mathrm{R} \widehat{\mathrm{OH}}+1 / 2 \mathrm{O}_{2} \stackrel{\text { Catalyst }}{\longrightarrow} \underset{\mathrm{R}}{\stackrel{\mathrm{O}}{\mathrm{N}}} \mathrm{OH}+\mathrm{H}_{2} \mathrm{O}$

B)

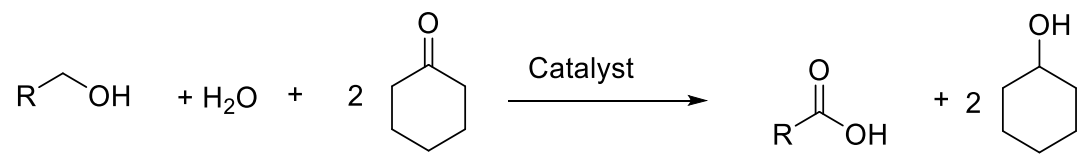

C)
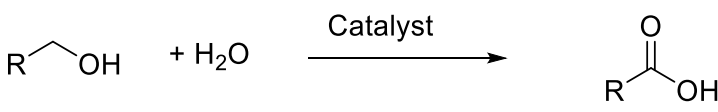

$2 \mathrm{H}_{2} \uparrow$

Non-poluting and valuable product

Figure 2.2 Different strategies for the synthesis of carboxylic acids

\section{Reactions using oxygen as the terminal oxidant}

An attractive approach to avoid the use of stoichiometric metal salts is the use of oxygen as oxidant in combination with a metal catalyst. The use of oxygen is very convenient as it is an abundant starting material. Especially interesting is the use of oxygen from air instead of pure $\mathrm{O}_{2}$ gas. The production of carboxylic acids using oxygen is mostly based in heterogeneous catalysts of ruthenium, ${ }^{[7]}$ gold, ${ }^{[8]}$ palladium or platinum on carbon or alumina supports. ${ }^{[5,9]}$ However, some homogeneous catalysts have recently been described. In 2014 a bis-NHC silver complex that efficiently catalyses the transformation of primary alcohols to carboxylic acids under aerobic basic conditions was reported (Figure 2.3). ${ }^{[10]}$ Interestingly, the authors describe that a careful choice of the reaction conditions (solvent, base, temperature) can finely control the aldehyde/carboxylic acid product selectivity. Apart from this system, other homogeneous catalysts based on noble, ${ }^{[11]}$ and earth abundant metals, ${ }^{[12,13]}$ have also been successfully applied. 

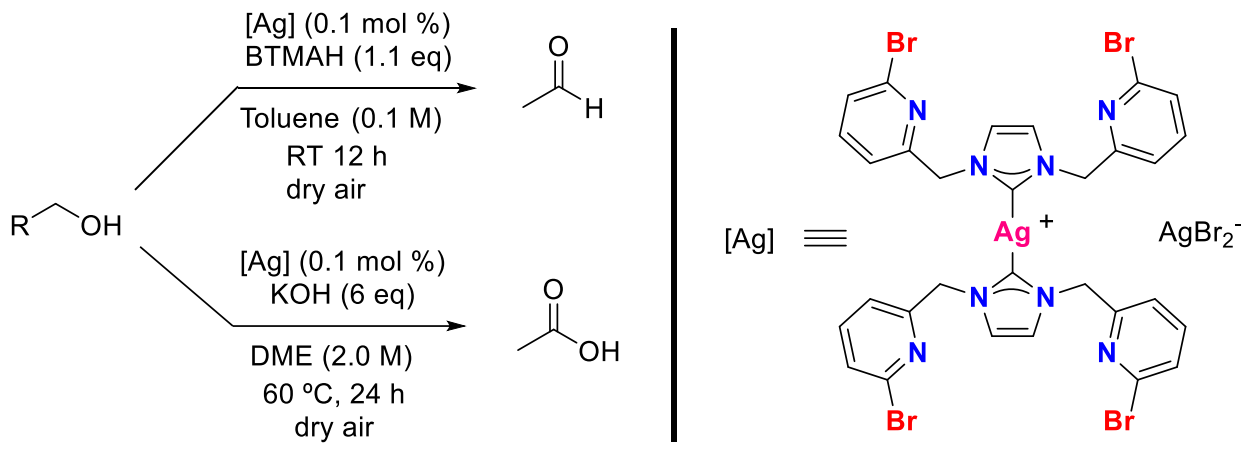

Figure 2.3 Silver catalysed selective oxidation of primary alcohols

Dehydrogenative oxidations using a sacrificial hydrogen acceptor

An alternative route for the oxidation of alcohols is the removal of hydrogen from an alcohol to obtain an oxidized product (an aldehyde, ketone or carboxylic acid). The dehydrogenative catalytic oxidation of alcohols can be seen as the reverse reaction of the classic hydrogen transfer catalysis (HT). In its original form, the HT reaction consists in the transfer two hydrogen atoms from a sacrificial secondary alcohol (usually 2-propanol) to a ketone mediated by a transition metal catalyst. The goal of $\mathrm{HT}$ is reducing a carbonyl compound to the corresponding alcohol (Figure 2.4).

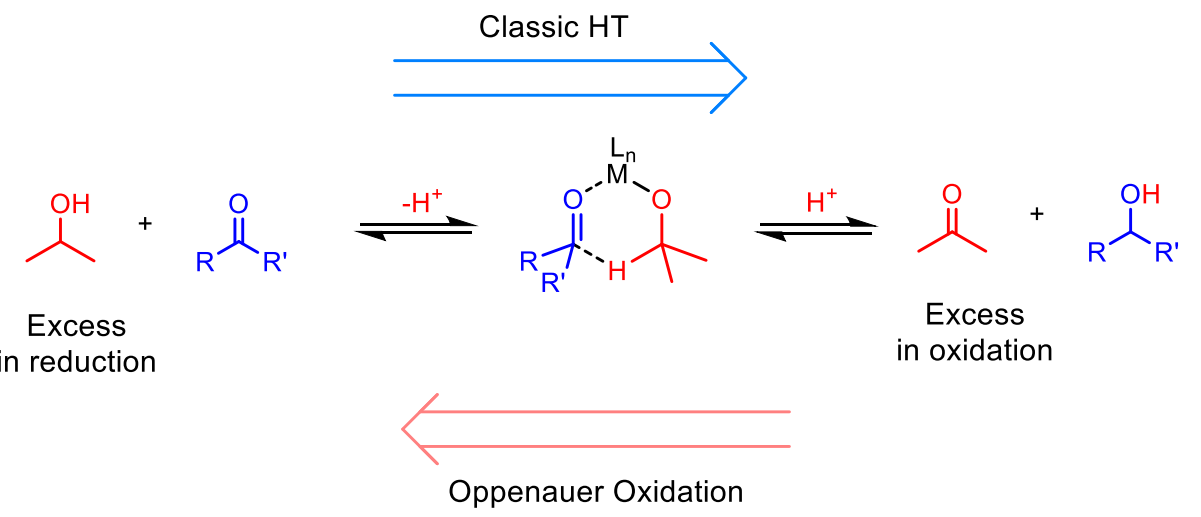

Figure 2.4 Classic transfer hydrogenation and Oppenauer oxidation

For the oxidation of alcohols, it is interesting make the system run for the reverse cycle, known as Oppenauer oxidation. ${ }^{[14]}$ In this reaction two hydrogens are transferred from a primary or secondary alcohol to an acceptor, yielding an aldehyde or a ketone and a sacrificial waste. Although acetone was the first acceptor used, other hydrogen acceptors such as alkenes can be used. ${ }^{[15]}$ Several groups have 
demonstrated transition metal catalysed systems that show Oppenauer-type alcohol oxidation reactivity. Although initial reports used metal complexes based on iridium or rhodium, ${ }^{[16]}$ ruthenium has become the most general metal used for this type of oxidations. $^{[17-20]}$

In general, the systems that are active in Oppenauer alcohol oxidations are limited to the synthesis of aldehydes and ketones. Nevertheless, some reports for the synthesis of carboxylic acids using this strategy can be found in the literature. In 2009 Grützmacher and co-workers reported a bifunctional Rh catalyst that catalyses the oxidation of primary alcohols to carboxylic acids (Figure 2.5). ${ }^{[21,22]}$ The reaction is carried in water and cyclohexanone (5 equivalents) is used as the hydrogen acceptor. Interestingly, the formed cyclohexanol could be transformed back to cyclohexanone by treatment with a diluted solution of hydrogen peroxide. The same group reported that fine-tuning of the catalyst structure can lead to the use of $\mathrm{O}_{2}$ from air as the hydrogen acceptor, ${ }^{[23]}$ which opens new possibilities in the oxidation of alcohols.
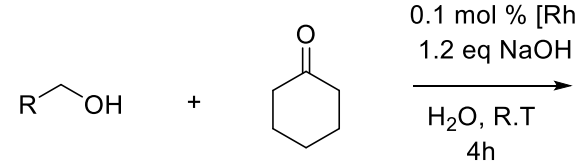<smiles>OC1CCCCC1</smiles>

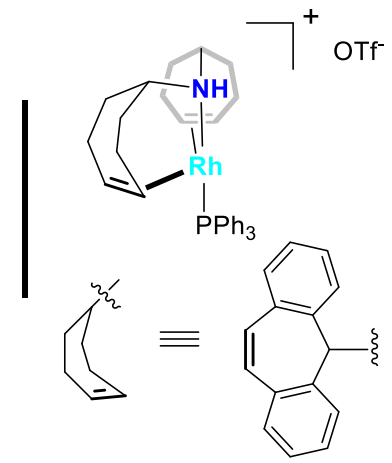

Figure 2.5 Dehydrogenative oxidation of primary alcohols in the presence of a hydrogen acceptor

\section{Acceptorless dehydrogenative oxidations}

A more suitable process for the oxidation of alcohols is the acceptorless dehydrogenation $(A D H)$. In this reaction a primary alcohol is converted into a oxidised product and the only by-product is molecular hydrogen, which is valuable itself as a high energy clean-fuel. The first example of ADH of an alcohol was published by Charman in 1966, who reported the oxidation of isopropanol to acetone with the concomitant liberation of $\mathrm{H}_{2}$ using rhodium trichloride in acidic media, ${ }^{[24,25]}$ preferably in the presence of stannous chloride. ${ }^{[26]}$ Then Dobson and 
Robinson reported more active systems of ruthenium and osmium complexes bearing perfluorocarboxylate ligands, ${ }^{[27]}$ which are able to oxidise a wide number of primary and secondary alcohols to aldehydes and ketones. Interestingly, this ruthenium catalyst can be immobilised onto polystyrene and reused. ${ }^{[28]}$ In particular the groups of Beller, ${ }^{[29]}$ Fujita and Yamaguchi, ${ }^{[30,31]}$ and Milstein ${ }^{[32]}$ have become leaders in the study of this kind of reactions.

In 2013 Milstein and co-workers reported the first catalyst that can dehydrogenate primary alcohols to generate the corresponding carboxylic acid in the absence of a sacrificial hydrogen acceptor (Figure 2.6). The reaction works under relatively mild conditions and in basic aqueous media, which is very convenient from a green chemistry viewpoint. The system is based on a ruthenium catalyst with a pincer ligand that promotes metal-ligand cooperation via aromatization/dearomatization of the pyridine-based pincer ligand. ${ }^{[33]}$ The reaction works at low catalyst loadings $(0.2 \mathrm{~mol} \%)$ and with good isolated yields and excellent selectivity. With this catalyst, a wide scope of carboxylic and di-carboxylic acids could be obtained in high purity after acidic treatment of the reaction media.

$$
\widehat{\mathrm{R}} \underset{\mathrm{NaOH}, \mathrm{H}_{2} \mathrm{O}}{[\mathrm{Ru}] \text { catalyst }}+\overbrace{\mathrm{O}}^{\Theta}+2 \mathrm{H}_{2} \uparrow
$$

Figure 2.6 Acceptorless dehydrogenation of alcohols to obtain carboxilic acid salts reported by Milstein

Since this first report, other systems based on non-cooperative and cooperative pincer systems of ruthenium, ${ }^{[34]}$ manganese or iron ${ }^{[35]}$ have been reported for the aqueous dehydrogenation of primary alcohols (I-III Figure 2.7). 


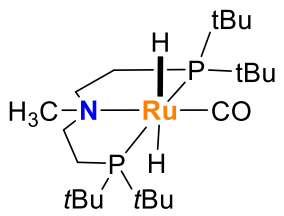

I

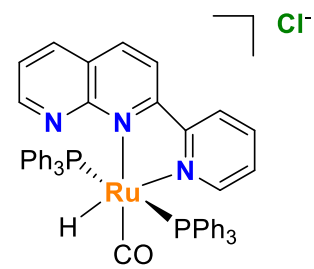

IV

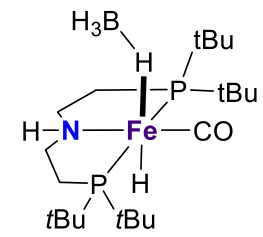

II

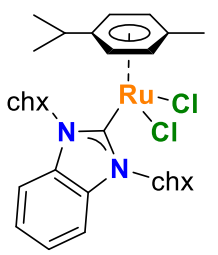

chx $=$ cyclohexyl

V

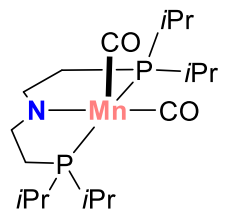

III

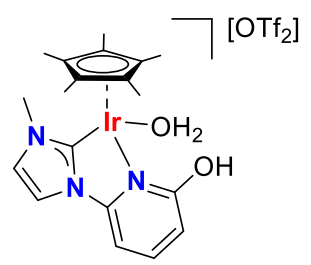

VI

Figure 2.7 Representative examples reported for the ADH of primary alcohols to carboxilic acids

Alternatively, other catalysts based on non-pincer ligands (IV and V, Figure 2.7) have been reported for this reaction, which can be viewed as an advantage, as the phosphorous pincer ligands usually require several reaction steps and give rise to air-sensitive complexes. ${ }^{[36,37]}$ In 2017, the group of Fujita and Yamaguchi reported for the first time the acceptorless dehydrogenation of alcohols to obtain carboxylic acids in water under neutral conditions (VI, Figure 2.7). Although the catalyst works at relatively high catalyst loading $(2 \mathrm{~mol} \%)$ and is based in a more expensive metal such as iridium, it constitutes the first example for the direct dehydrogenative generation of carboxylic acids without added base.

The recycling of the catalyst for the $A D H$ of primary alcohols to obtain carboxylic acids has been scarcely studied. The development of highly active and recyclable catalysts is key for the success of this methodology in an industrial scale. The use of heterogeneous systems for the ADH synthesis of carboxylic acids will limit the cost related to the use of catalysts based on expensive noble metals. As far as we know, there are only two publications reporting the heterogeneously catalysed transformation of alcohols to carboxylic acids in water and in the absence of any acceptor. These systems, based on $\mathrm{Rh}^{[38]}$ and $\mathrm{Pd}^{[39]}$ present a wide substrate scope and good recyclability (5 runs) but have the following drawbacks: i) both of them need to work under reduced pressure to remove the hydrogen from the reaction media and drive forward the reaction. ii) both of them use a high catalyst loading (10-20 mol \%). 
In this chapter, a new approach to carry out the ADH of primary alcohols is described. Our system is based on a Ru complex coordinated to a NHC ligand with a (poly)aromatic pendant group that allows its immobilisation onto reduced graphene oxide, giving rise to a recyclable heterogeneous system (Figure 2.8).

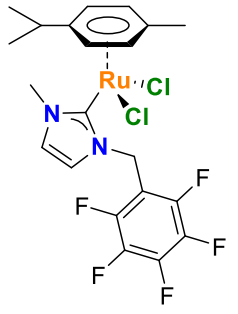

$1 \mathrm{~A}$

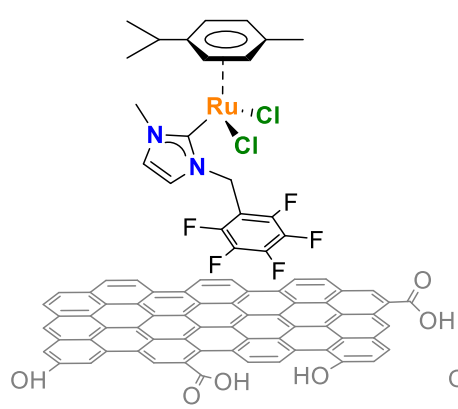

1A-rGo

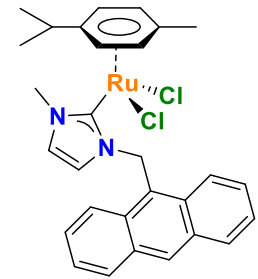

2B

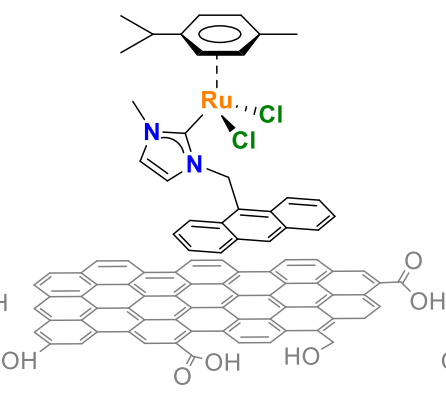

2B-rGO

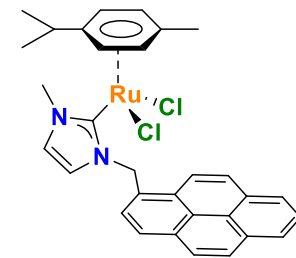

3C

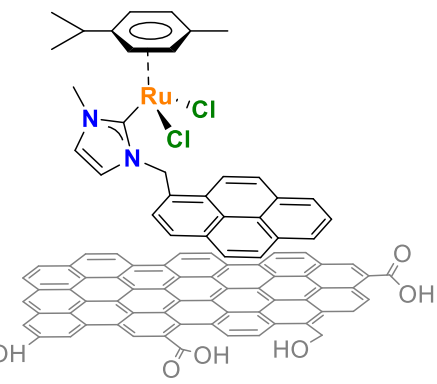

3C-rGo

Figure 2.8 Catalysts tested in this chapter

The influence of the aromatic group over the catalysis and the recyclability is analysed. Additionally, a plausible mechanism is proposed based on Density Functional Theory (DFT) calculations and experimental evidence obtained by Nuclear Magnetic Resonance (NMR) and Electrospray Ionization-Mass Spectrometry (ESI-MS). 


\subsection{RESULTS AND DISCUSSION}

\subsubsection{Synthesis of ruthenium catalysts and hybrid ruthenium materials}

One of the aims of this chapter is to study the influence of the aromatic group in the NHC ligand on the grafting efficiency of the molecular complex onto rGO. To this purpose, three ruthenium complexes featuring different polyaromatic tags were synthesised.

Starting from three different imidazolium salts with a pentafluorobenzyl group $(\mathbf{A}),{ }^{[40]}$ an anthracene tag $(\mathbf{B})^{[41]}$ or a pyrene tag $(\mathbf{C}),{ }^{[42]}$ the corresponding $\left[\mathrm{Ru}(\mathrm{p}-\mathrm{cym})(\mathrm{NHC}) \mathrm{Cl}_{2}\right]$ complexes $\mathbf{1 A}, \mathbf{2 B}$, and $\mathbf{3} \mathbf{C}^{[42]}$ were prepared. The three complexes were obtained by transmetallation with $\mathrm{Ag}_{2} \mathrm{O}$, thus avoiding the use of strong bases and the isolation of air and moisture sensitive carbene species (Figure 2.9). ${ }^{[43]}$ The two new complexes $\mathbf{A A}$ and $\mathbf{2 B}$ were fully characterised by ${ }^{1} \mathrm{H}$ NMR, ${ }^{13} \mathrm{C}$ NMR, ESI-MS, High Resolution Mass Spectrometry (HRMS) and elemental analysis; and suitable single crystals for X-Ray Diffraction studies were obtained for $\mathbf{1 A}$ and $\mathbf{3 C}$. The first evidence that the metalation had taken place was the disappearance of the acidic signal due to the NCHN proton of the imidazolium salt in the ${ }^{1} \mathrm{H}$ NMR spectrum. The formation of complexes $\mathbf{1 A}$ and $\mathbf{2 B}$ was further confirmed by ${ }^{13} \mathrm{C}$ NMR, where the signals for the carbenic carbons were observed at 175.9 and $172.6 \mathrm{ppm}$ respectively. All the information regarding the characterisation of new compounds is included in chapter 6 .

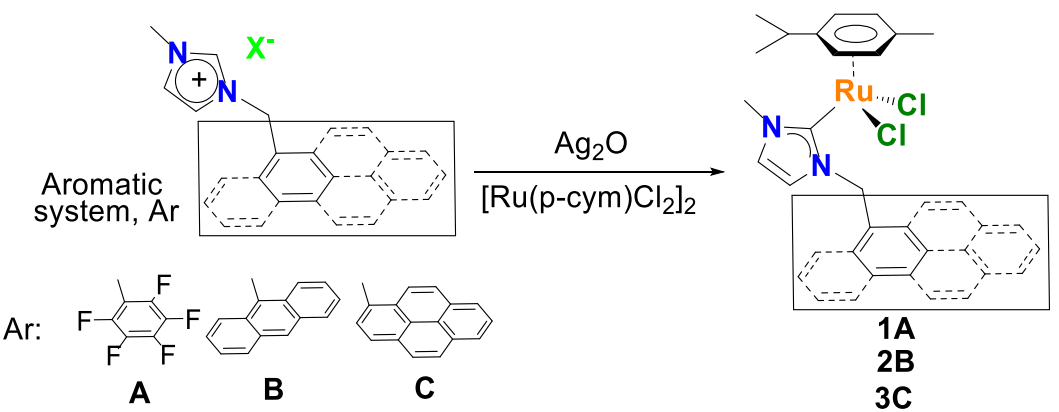

Figure 2.9 Synthesis of Ru complexes $1 \mathrm{~A}, \mathbf{2 B}$ and $\mathbf{3 C}$

Complexes 1A 2B, and 3C, were grafted onto reduced graphene oxide using a methodology previously described in our research group. ${ }^{[42,44]}$ The graphene was first sonicated in dichloromethane for 30 minutes and then the molecular complex was added. The mixture was sonicated for 10 minutes and further stirred at room temperature for 24 hours. After that, the black solid was filtered off and washed 
with dichloromethane, obtaining the hybrid materials 1A-rGO, 2B-rGO and 3C-rGO as black solids (Figure 2.10 ).
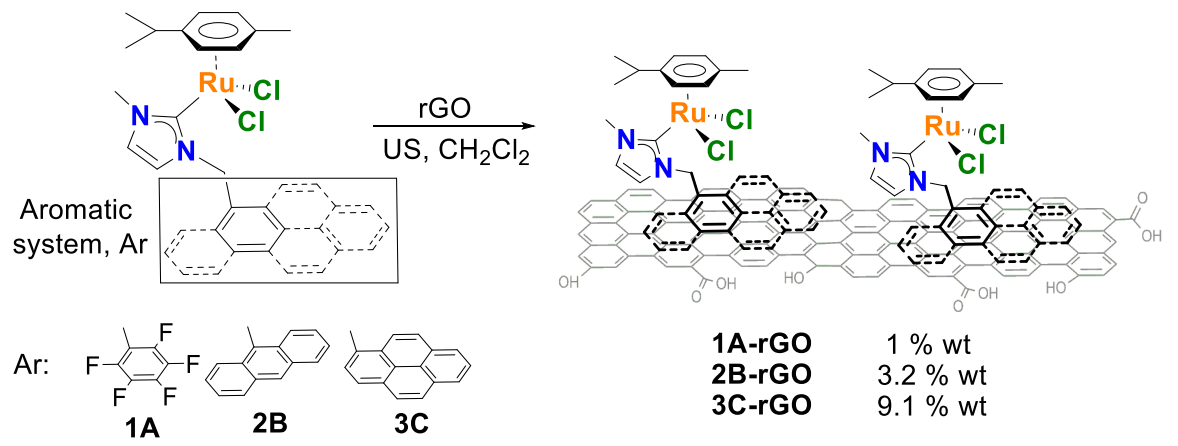

Figure 2.10 Grafting of ruthenium molecular complexes onto rGO

The three materials were characterised by High Resolution Transmission Microscopy (HRTEM) and Ultraviolet-Visible spectroscopy (UV/Vis), and the exact metal content of ruthenium was determined by digestion of the samples in nitric acid followed by ICP-MS. The amount of metal complex on the surface of rGO increases with the number of aromatic rings, which gives an idea of the strength of the interaction between the polyaromatic tag and the graphene (Figure 2.10). The pentafluorobenzyl containing compound $\mathbf{1 A}$ is barely retained on the surface of rGO. On the contrary $\mathbf{3 C}$, with a pyrene tag, is firmly bonded to the surface of graphene due to the stronger $\pi-\pi$ interaction.

The UV/Vis measurements revealed that the spectrum of the hybrid material 2B-rGO (black curve) contains the characteristic bands of the molecular complex 2B (Figure 2.11). The spectra of both (2B and 2B-rGO) show the anthracene UV/Vis characteristic signals assigned to the $\pi-\pi^{*}$ transition. ${ }^{[45,46]}$ Hence, UV/Vis spectroscopy confirms the immobilisation of the molecular complexes on the surface of graphene. 


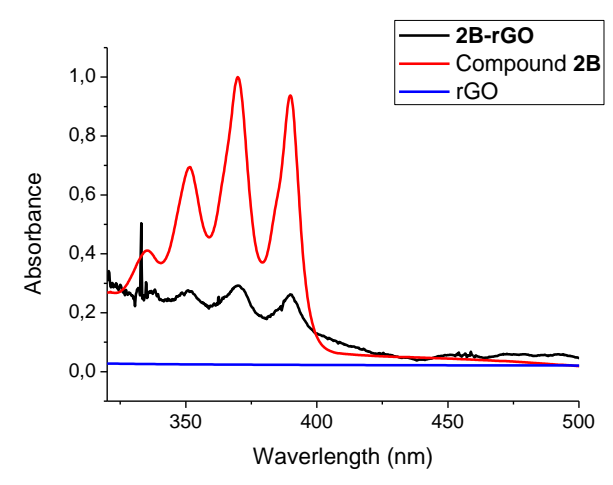

Figure 2.11 UV/Vis spectra for 2B, 2B-rGO and rGO. The samples were suspended in DMF and sonicated before recording the spectrum. The molecular complex was measured in a solution of DMF $\left(10^{-6} \mathrm{M}\right)$

HRTEM images for 2B-rGO (Figure 2.12 a and b) show the characteristic morphology of graphene. The support material is composed of carbon layers that contain defects in the form of wrinkles. This effect is more prominently observed at the edges. It is important to notice that the morphology of the graphene is preserved after the immobilisation process. Metal nanoparticles were not observed, indicating the molecular nature of the species anchored on the graphene. The elemental mapping (Figure 2.12, d) shows the homogeneous distribution of the metal along all the surface of the graphene and not only on the defects. (compare red dots in $d$ versus the morphology of the graphene of the STEM image shown in c). Additionally, the analysis by Energy-Dispersive X-Ray spectroscopy (EDS) further confirms the presence of ruthenium onto $\mathrm{rGO}$. 

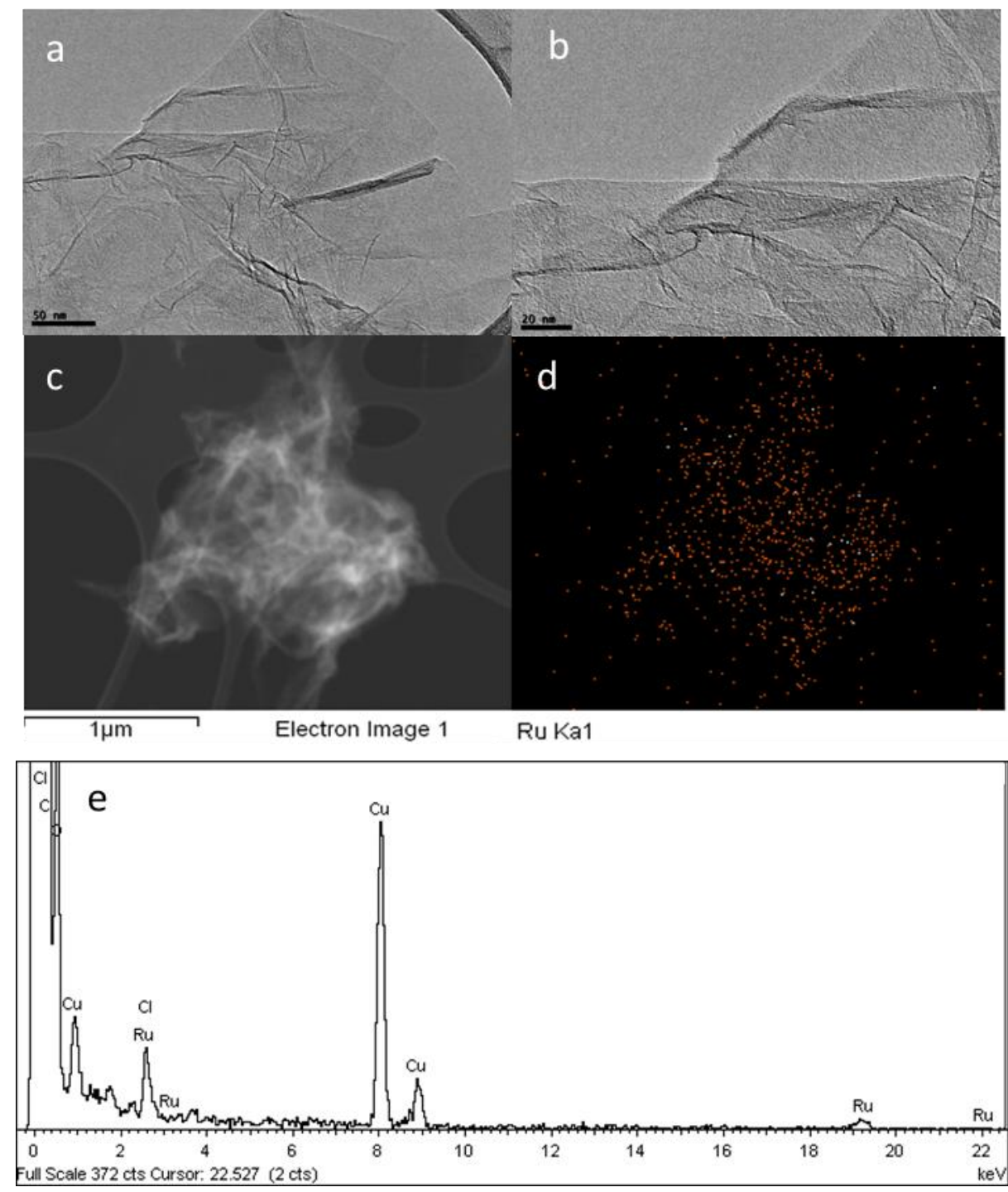

Figure 2.12 HRTEM (a, b), STEM (c), elemental mapping (d) and EDS spectrum of 2B-rGO

\subsubsection{Catalytic studies: acceptorless alcohol oxidation}

The molecular complexes $\mathbf{1 A}, \mathbf{2 B}$ and $\mathbf{3 C}$ and the related hybrid materials $\mathbf{1 A - r G O}$, 2B-rGO and $\mathbf{3 C}-\mathbf{r G O}$ were tested as catalysts for the ADH of primary alcohols in aqueous media. In a first set of experiments, the catalytic reactions were performed using benzyl alcohol as the model substrate, in the presence of 1 equivalent of caesium carbonate and heating at reflux temperature for 24 hours in an open system. Conversions of benzyl alcohol were determined using a gas chromatograph with a Flame Ion Detector (GC-FID), and yields were calculated by ${ }^{1} \mathrm{H}$ NMR after 
acidification of reaction mixture and extraction with ethyl acetate (see chapter 6 for more details). The results for the optimization of the reaction conditions are included in Table 2.1

Table 2.1 Optimization of reaction conditions for the dehydrogenative oxidation of benzyl alcohol

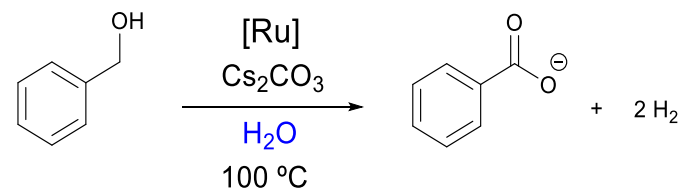

\begin{tabular}{cccc}
\hline Entry & Catalyst & {$[\mathrm{Ru}] \mathrm{mol} \%$} & Conversion (\%) $^{\mathrm{a}}$ \\
\hline 1 & rGO & - & 0 \\
2 & 1A & 0.2 & 43 \\
3 & 1A-rGO & 0.1 & 65 \\
4 & 1A-rGO & 1 & $98(87)$ \\
\hline 5 & 2B & 2 & 44 \\
6 & 2B-rGO & 0.1 & $89(76)$ \\
7 & 2B-rGO & 1 & $100(95)$ \\
\hline 8 & 3C & 0.1 & 11 \\
9 & 3C-rGO & 0.1 & $74(70)$ \\
10 & 3C-rGO & 1 & $100(93)$ \\
\hline
\end{tabular}

Reaction conditions: benzyl alcohol $(0.5 \mathrm{mmol}), \mathrm{Cs}_{2} \mathrm{CO}_{3}$ (1 eq.), catalyst, $10 \mathrm{~mL}$ of water at $100{ }^{\circ} \mathrm{C}$ for $24 \mathrm{~h}$. [a] Conversion determined by GC analysis using anisole as standard. Isolated yield after acidification in parenthesis determined by ${ }^{1} \mathrm{H}$ NMR using trimethoxy benzene as standard.

A blank experiment using only $\mathrm{rGO}$ and caesium carbonate was performed to analyse any catalytic activity caused by the support (Table 2.1, entry 1 ). The result was the complete recovery of the starting material. This experiment discards rGO as the real catalyst, acting as carbocatalyst. In general, carbocatalysts are based on carbonaceous materials with numerous oxygenated functionalities and require high catalyst loadings (2-200 wt \%), and none of these requirements are fulfilled in this case. ${ }^{[47,48]}$ The molecular complexes $\mathbf{1 A}, \mathbf{2 B}$ and $\mathbf{3 C}$ and the related hybrid materials 1A-rGO, 2B-rGO and 3C-rGO were active in the dehydrogenation of benzyl alcohol to benzoate. The formation of by-products such as esters or hemiacetals was not observed, and just a small amount of benzaldehyde (ca. 5-10 \%) was found at the end of the reaction (vide infra), indicating that these catalysts are selective for alcohol oxidation. 
The molecular complexes accounted for low to moderate conversions of benzylalcohol after 24 hours in refluxing water at a catalyst loading of $0.1-2 \mathrm{~mol} \%$ (Table 2.1, entries 2, 5 and 8). When we tested the related hybrid materials under the same catalyst loading the conversions increased (compare entries $2 \& 3,5 \& 6$ and $8 \& 9$ in Table 2.1), and complete conversions were obtained when the loading was increased up to $1 \mathrm{~mol} \%$ (entries 4,7 and 10). This result suggests that the molecular complexes are active in the dehydrogenation of alcohols, but there is a competing deactivation effect. Although the nature of this deactivation effect is unknown, it is mitigated when the complex is grafted onto reduced graphene oxide. The immobilisation of the three complexes onto rGO provides a catalytic benefit, probably because of the stabilisation of the catalytic active species.

After the optimization of the reaction conditions, catalysts $\mathbf{1 A - r G O}, \mathbf{2 B - r G O}$, and $\mathbf{3 C}$ rGO were tested in the dehydrogenation of different benzyl alcohols (Table 2.2). The three heterogenised catalysts were active in the formation of carboxylic acid salts at low catalyst loading $(0.1 \mathrm{~mol} \%)$, affording good to excellent conversions. No changes in selectivity were observed after the immobilisation of the molecular species and after the work-up the carboxylic acid was the only observed product. All the products were isolated after acidification at the end of the reaction and the carboxylic acids were identified by ${ }^{1} \mathrm{H}$ NMR spectroscopy.

Electron donating and electron withdrawing groups are compatible with the catalytic system. In general, better results were obtained in the case of substrates with $p$-substituted electron withdrawing groups. The best outcome was obtained using ( $p$-trifluoromethyl)benzyl alcohol as substrate (Table 2.2, entries 4,8 and 12). The time-conversion profile for this substrate using catalyst 2B-rGO is included in chapter 6 (Figure 6.31). 
Table 2.2 Dehydrogenation of substituted benzyl alcohols in water

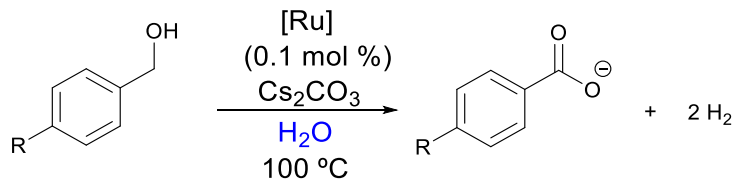

\begin{tabular}{cccc}
\hline Entry & Catalyst & $\mathbf{R}$ & Conversion (\%) $^{\mathbf{a}}$ \\
\hline 1 & 1A-rGO & $\mathrm{Me}$ & $90(85)$ \\
2 & 1A-rGO & $\mathrm{Cl}$ & 75 \\
3 & 1A-rGO & $\mathrm{Br}$ & 85 \\
4 & 1A-rGO & $\mathrm{CF}_{3}$ & 89 \\
\hline 5 & 2B-rGO & $\mathrm{Me}$ & 88 \\
6 & 2B-rGO & $\mathrm{Cl}$ & $82(80)$ \\
7 & 2B-rGO & $\mathrm{Br}$ & $99(90)$ \\
8 & 2B-rGO & $\mathrm{CF}_{3}$ & $100(92)$ \\
\hline 9 & 3C-rGO & $\mathrm{Me}$ & 76 \\
10 & 3C-rGO & $\mathrm{Cl}$ & 73 \\
11 & 3C-rGO & $\mathrm{Br}$ & 88 \\
12 & 3C-rGO & $\mathrm{CF}_{3}$ & $97(93)$ \\
\hline
\end{tabular}

Reaction conditions: substrate $(0.5 \mathrm{mmol}), \mathrm{Cs}_{2} \mathrm{CO}_{3}(1 \mathrm{eq})$, catalyst $(0.1 \mathrm{~mol} \%), 10 \mathrm{~mL}$ of water at $100{ }^{\circ} \mathrm{C}$ for $24 \mathrm{~h}$. [a] Conversions determined by GC analysis using anisole as the standard. Isolated yield after acidification in parenthesis determined by ${ }^{1} \mathrm{H}$ NMR using trimethoxy benzene as standard.

\section{Recyclability studies}

Given the high activity of the solid materials 1A-rGO, 2B-rGO and 3C-rGO, we decided to test the recyclability of the three catalysts. These experiments were done using ( $p$-trifluoromethyl)benzyl alcohol as the substrate in the presence of 1 equivalent of caesium carbonate. After $8 \mathrm{~h}$ ( $c a$. conversion $50 \%$ ) the reaction was stopped and analysed by GC, and the solid catalyst was recovered by decantation, washed thoroughly with water and used again. Before starting a new cycle, addition of another equivalent of caesium carbonate was essential to make the reaction run.

The three materials gave excellent results in terms of recyclability (especially considering the low catalyst loading used, $0.1 \mathrm{~mol} \%$ ), and could be reused up to five times without any observed decrease in activity. However, we observed differences between catalysts from run six (Figure 2.13). 
$[\mathrm{Ru}]$
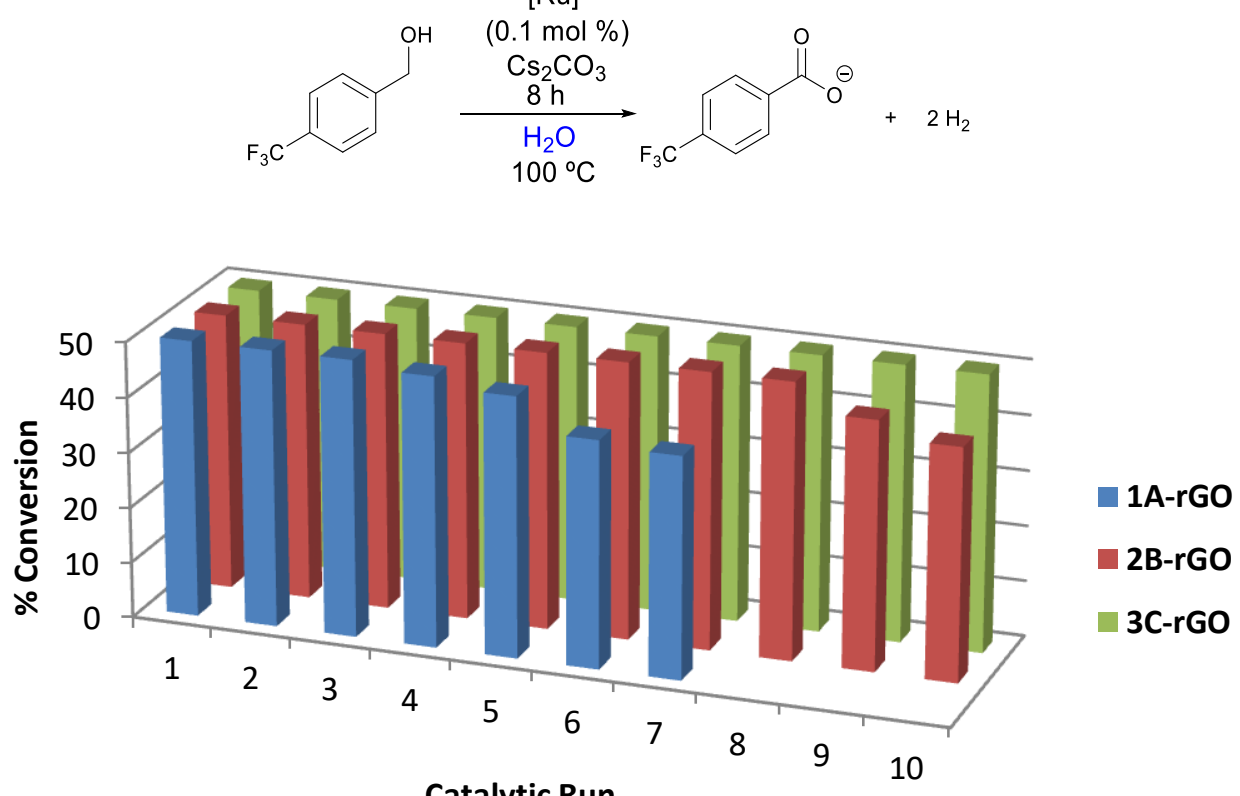

Catalytic Run

Figure 2.13 Recycling experiments for the dehydrogenation of ( $p$-trifluoromethyl)benzyl alcohol using hybrid materials 1A-rGO-3C-rGO. Conversions determined by GC analysis after $8 \mathrm{~h}$ reaction

The endurance of the catalytic system is significantly influenced by the benzylic $\mathrm{N}$-substituent at the imidazolylidine ring. Thus, 1A-rGO was recycled for five times without any decrease in activity, but then in runs six and seven the conversion decreased from $50 \%$ to $43 \%$ and $40 \%$ respectively. Similarly, 2B-rGO was reused eight times without any alteration in the performance, but in runs nine and ten the conversion dropped to $40 \%$. The best material in terms of recyclability was $\mathbf{3 C}$-rGo, which could be recycled up to ten times without any decrease in activity. These results indicate that the strength of the interaction between the aromatic tag at the imidazolylidine ring and the $\mathrm{rGO}$ is dramatically affected by the number of aromatic rings. Hence, the material $\mathbf{1 A - r G O}$ with just one aromatic ring is more easily leached from the surface of rGO (and less recyclable) than 3C-rGO, which features a pyrene group.

To study if the deactivation of the catalyst was caused by leaching of the molecular complex from the surface, the Maitlis Hot Filtration Test was performed. ${ }^{[49]}$ When this experiment was done for the three hybrid catalysts, different results were 
obtained. For the material 3C-rGO with a pyrene tag, the reaction was set up with ( $p$-trifluoromethyl)benzyl alcohol as model substrate, caesium carbonate and water, and stired at reflux for $8 \mathrm{~h}$ (conversion $45 \%$ ). After this time, the hybrid material 3C-rGO was removed by filtration and the filtrate was reacted for $12 \mathrm{~h}$. In parallel, the solid catalyst was reacted with more alcohol and base. Analysis by GC showed that, for the filtrate, the conversion remained unaltered, but a $42 \%$ conversion was observed for the solid catalyst, indicating the heterogeneous nature of $\mathbf{3 C}-\mathrm{rGO}$ and confirming the absence of leached species in the reaction media. On the contrary, when the experiment was performed under the same reaction conditions but using 1A-rGO or 2B-rGO, the catalytic reaction continued in the solution after filtration at $100{ }^{\circ} \mathrm{C}$, indicating the homogeneous nature of the process. This experiment confirms that while 3C-rGO acts as heterogeneous catalyst, 1A-rGO and 2B-rGO operate under a catch and release mechanism. When the reaction is heated at $100{ }^{\circ} \mathrm{C}$ the homogeneous complexes are desorbed from the rGO surface and catalyse the reaction in the homogeneous phase. Then, when the reaction is allowed to reach room temperature the complexes are re-immobilised onto $\mathrm{rGO}$, so they can be separated and reused. During this return to the rGO surface some of the desorbed ruthenium is lost, which explains a lower quality in terms of recyclability.

To further confirm leaching as the cause of deactivation, the Ru content in the solid catalyst at the end of the recycling experiment was analysed by ICP-MS (Table 2.3). For the hybrid material $3 \mathrm{C}$-rGO, the results showed a ruthenium content without considerable changes after 10 runs ( $9.1 \mathrm{wt} \%$ initially and $9.0 \mathrm{wt} \%$ at the end). However, the situation was different with 1A-rGO and 2B-rGO, where there was a clear decrease in metal loading (from 1 wt \% to 0.6 wt \% for $\mathbf{1 A - r G O}$ and from $3.2 \mathrm{wt} \%$ to $2.5 \mathrm{wt} \%$ for $2 \mathrm{~B}-\mathrm{rGO})$. These results point to leaching as the main effect causing deactivation of 1A-rGO and 2B-rGO, although other deactivation mechanisms may also be operating.

Table 2.3 ICP-MS data before and after the catalytic experiments

\begin{tabular}{|c|c|c|c|}
\cline { 2 - 4 } \multicolumn{1}{c|}{} & 1A-rGO & 2B-rGO & 3C-rGO \\
\hline Initial Ru content & $9.1 \mathrm{wt} \%$ & $3.2 \mathrm{wt} \%$ & $1 \mathrm{wt} \%$ \\
\hline $\begin{array}{c}\text { Ru content after } \\
\text { catalysis }\end{array}$ & $9.0 \mathrm{wt} \%$ & $2.5 \mathrm{wt} \%$ & $0.6 \mathrm{wt} \%$ \\
\hline
\end{tabular}

In order to identify the true active species, the three hybrid materials were analysed by TEM after the recycling experiments. The TEM micrographs of 1A-rGO (Figure $2.14 \mathrm{a}$ and $\mathrm{b})$ show that no nanoparticles have been formed after seven catalytic 
runs. Apparently, there is no modification of the support morphology and the material preserves its single layer nature. Additionally, the EDS elemental mapping (Figure 2.14, d) shows the homogeneous distribution of ruthenium on the surface of rGO after the recycling experiment. Similar analyses were obtained with 2B-rGO and 3C-rGO after the recycling experiments. These TEM results rule out the formation of metal nanoparticles as active catalytic species in the reaction and indicate the molecular nature of the catalyst anchored onto rGO.

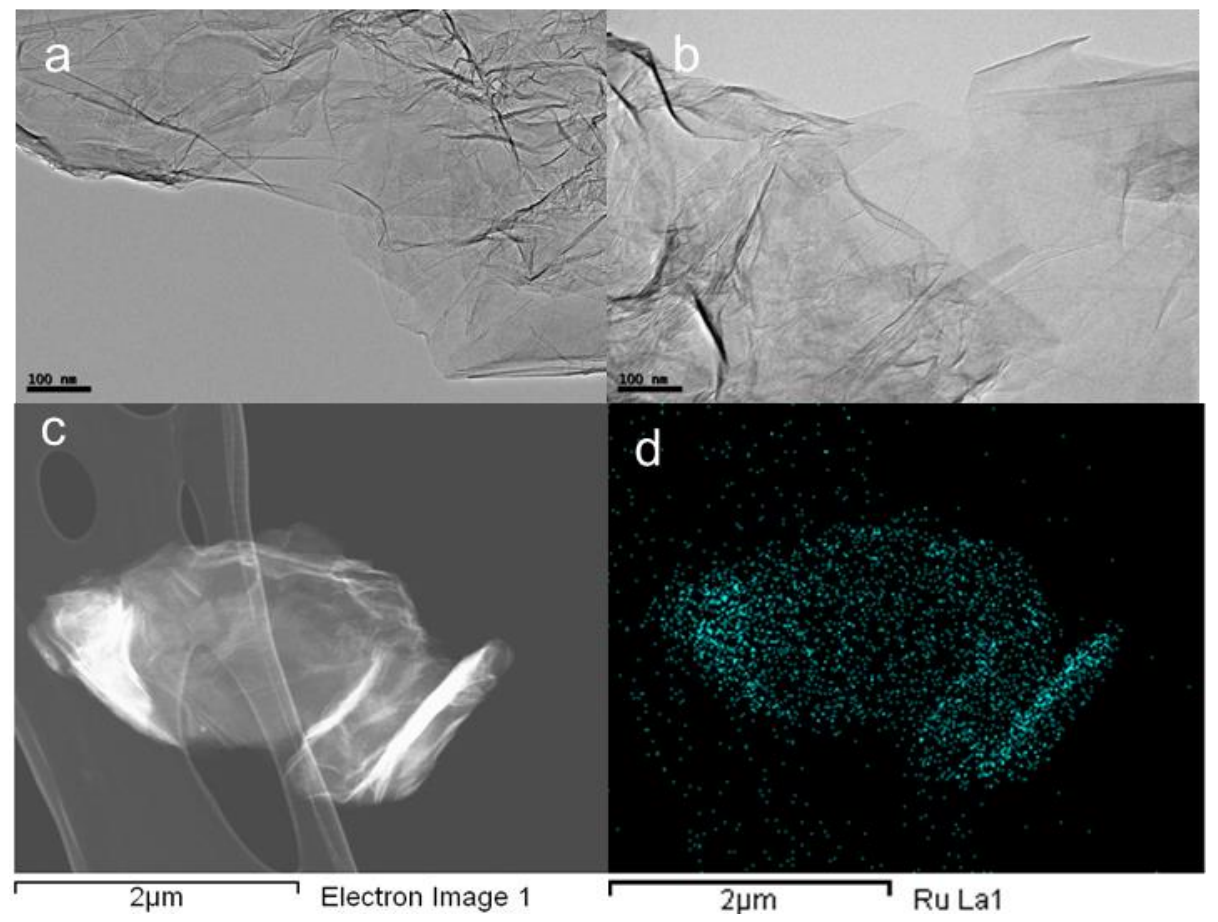

Figure 2.14 HRTEM images of 1A-rGO before (a) and after (b) seven catalytic runs. STEM (c) and EDS elemental mapping (d) images showing the homogeneous distribution of ruthenium after the seventh run

\section{Mechanism}

The system used for the catalytic dehydrogenation of alcohols (a molecular complex anchored onto rGO) constitutes an excellent opportunity to study and understand the mechanism of a heterogeneous catalyst with the aid of techniques usually applied to homogeneous catalysis. 
A plausible complete mechanism for the transformation of benzyl alcohol in the benzoate salt is depicted in Scheme 2.1. The mechanism proposal is based on experimental evidences obtained by ${ }^{1} \mathrm{H}$ NMR and ESI-MS. This mechanism was also supported by DFT theoretical calculations performed in collaboration with Dr. Miguel Baya, at Zaragoza University (Spain).

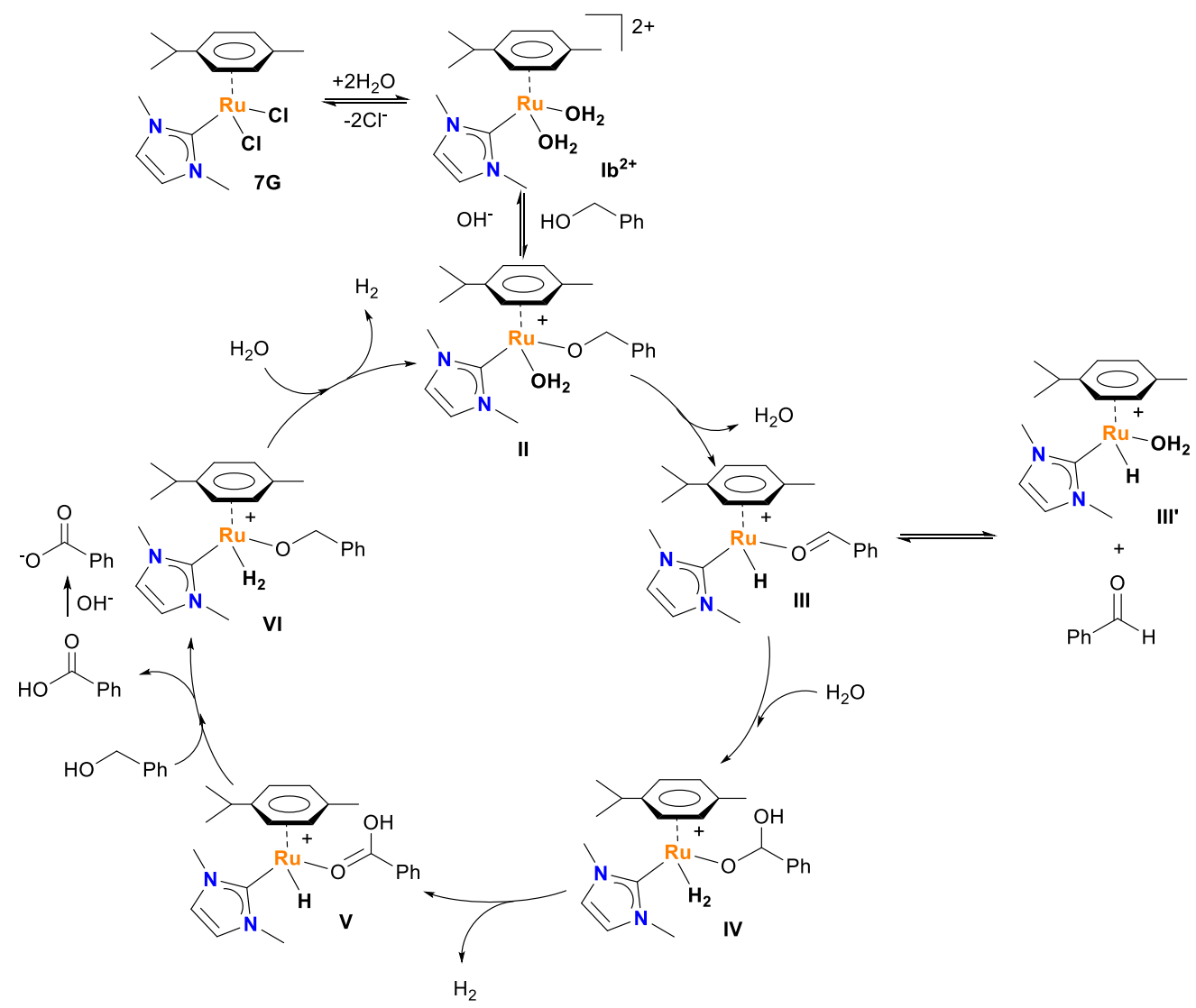

Scheme 2.1 Proposed mechanism for the dehydrogenation of primary alcohols using $\left[\mathrm{Ru}(p\right.$-cym $\left.)(\mathrm{NHC}) \mathrm{Cl}_{2}\right]$ complexes

The first step of the proposed mechanism is the substitution of one or two of the chlorides ligands for one/two molecules of water. This speciation was studied by $\mathrm{NMR}$ using a $\left[\mathrm{Ru}(p\right.$-cym $\left.)(\mathrm{NHC}) \mathrm{Cl}_{2}\right]$ complex with a simpler carbene ligand, 7G. ${ }^{[50]}$ For the ESI-MS experiments $\mathbf{1 A}$ was selected as a representative member of the family of catalysts herein described.

Complex $7 G$ displays broad signals in the ${ }^{1} \mathrm{H}$ NMR spectrum in $\mathrm{D}_{2} \mathrm{O}$ at room temperature (Figure 2.15, top). This broadening is consistent with the coexistence of 
different species in equilibrium. ${ }^{[51]}$ The spectrum is substantially simplified by the addition of base (Figure 2.15, bottom). Only one set of signals is observed, probably because the equilibrium is shifted to the formation of the neutral complex Ic, which features two hydroxyl ligands. However, the exact nature and structure of these species could not be unambiguously assigned by ${ }^{1} \mathrm{H}$ NMR spectroscopy.

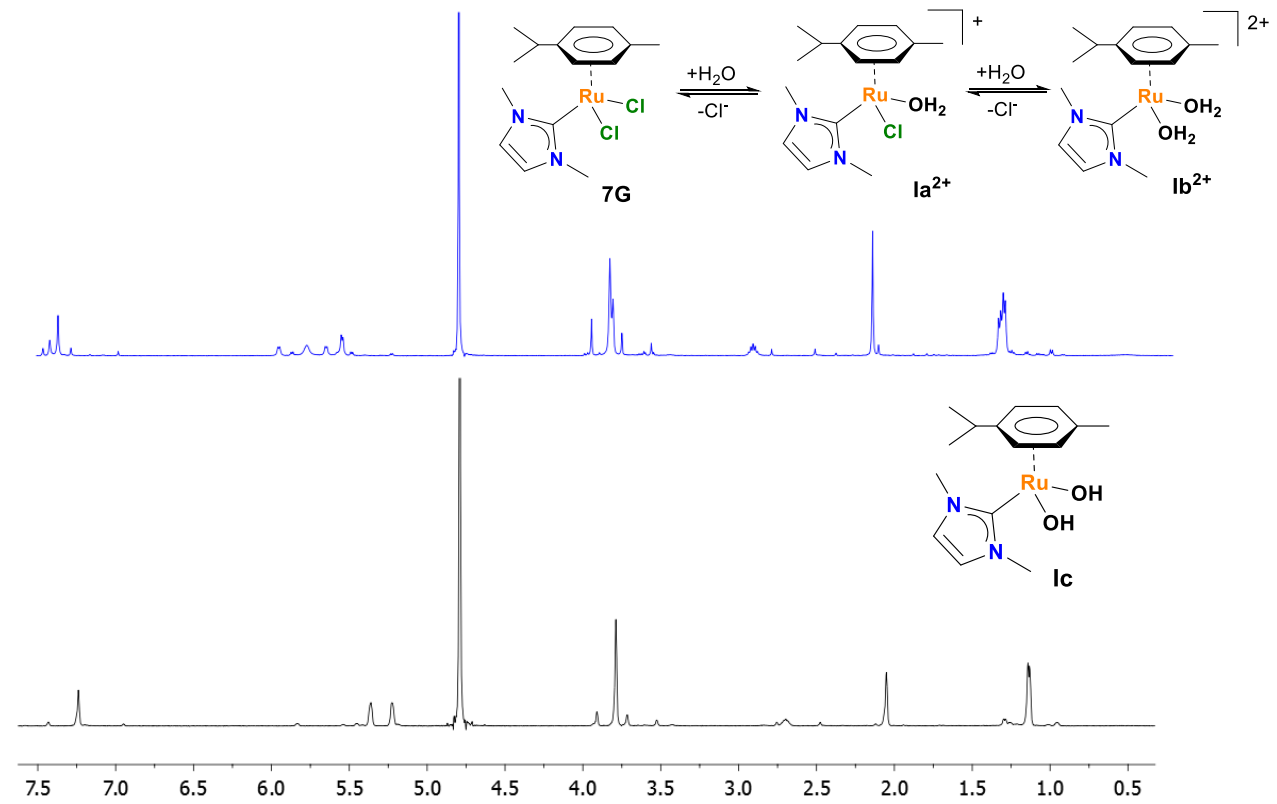

Figure 2.15 ${ }^{1} \mathrm{H}$ NMR spectra showing the speciation of 7G in neutral (top) and basic (bottom) $\mathrm{D}_{2} \mathrm{O}$

To gain more insights about the speciation of $\mathrm{Ru}$ complexes in water ESI-MS experiments were performed. The mass spectrum of aqueous solutions of $1 \mathrm{~A}$ is dominated by species lacking chlorine ligands as judged by their $\mathrm{m} / \mathrm{z}$ values as well as their characteristic isotopic pattern (Figure 2.16, top). Two major peaks at $\mathrm{m} / \mathrm{z}=249.0$ and $\mathrm{m} / \mathrm{z}=515.1$ are observed that formally correspond to $[\mathbf{1 A}-2 \mathrm{Cl}]^{2+}$ and $[1 \mathrm{~A}-2 \mathrm{Cl}+\mathrm{OH}]^{+}$. As the $\mathrm{pH}$ is raised by the addition of $\mathrm{NaOH}$, ionic species featuring $\mathrm{Ru}-\mathrm{OH}$ functional groups become more intense. As illustrated in Figure 2.16 (bottom), the $[1 \mathrm{~A}-2 \mathrm{Cl}+\mathrm{OH}]^{+}$and $[1 \mathrm{~A}-2 \mathrm{Cl}+2 \mathrm{OH}+\mathrm{Na}]^{+}$cations (this latter is the base peak) are the main species observed in the ESI-MS spectrum at pH 12. 


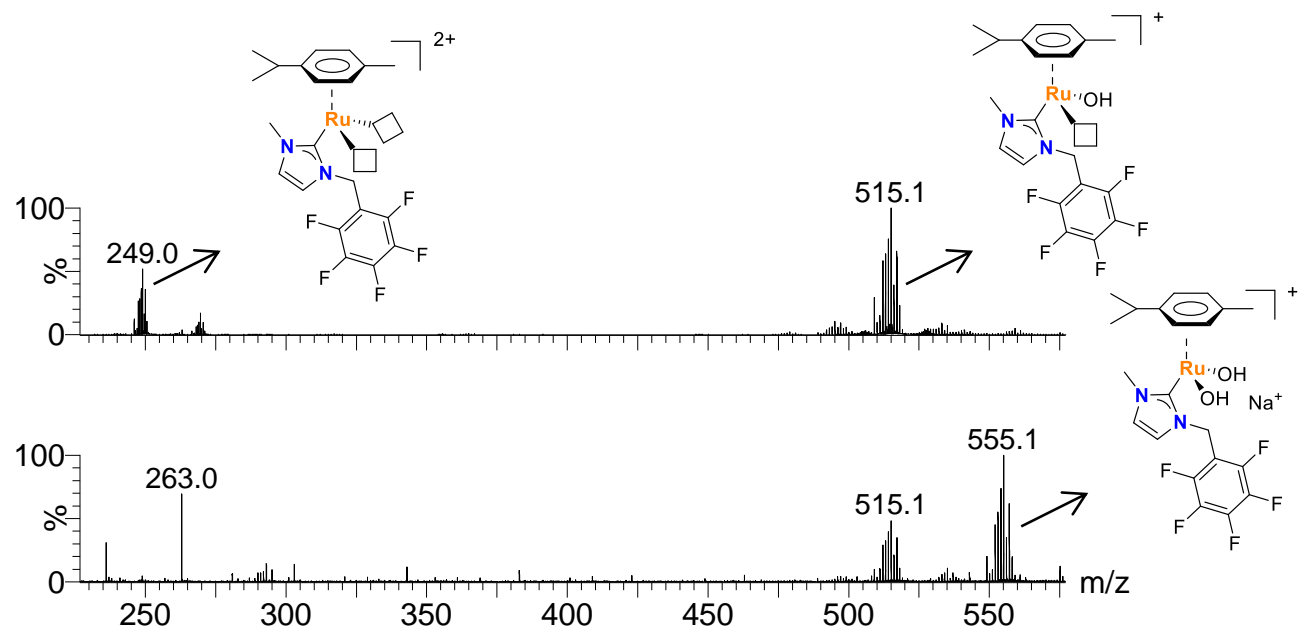

Figure 2.16 ESI-MS spectra of aqueous solution of $\mathbf{1 A}$ at $\mathrm{pH} 8$ (top) and 12 (bottom)

With these evidences in hand, we next explored the behaviour of $1 \mathrm{~A}$ in the presence of the substrate. Upon addition of 1 equivalent of benzyl alcohol to a solution of $\mathbf{1 A}$ in basic water the ESI-MS spectra displayed signals at $\mathrm{m} / \mathrm{z} 605.1$ and 619.1, which corresponds with the Ru-alcoxo and Ru-carboxilate species respectively (II and $\mathbf{V}$ in Scheme 2.1). The reaction of 7G with benzyl alcohol under basic conditions yields the Ru-alcoxo complex II, which starts the catalytic cycle. Complex II was characterised by ESI-MS as [II- $\left.\mathrm{H}_{2} \mathrm{O}\right]$. Then, a $\beta$-hydride elimination generates III. Complex III was indirectly identified by ESI-MS as [III-PhCHO]. So that, the collision induced dissociation (CID) spectrum of the $\left[\mathrm{II}-\mathrm{H}_{2} \mathrm{O}\right]$ species was recorded (Figure 2.17). The spectrum shows the formation of the Ru-hydride species III' along with the formation of benzaldehyde (Figure 2.17). 


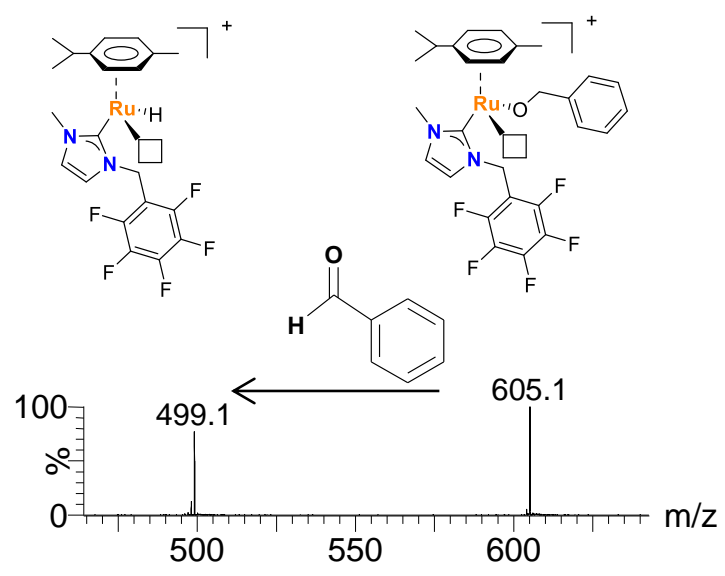

Figure 2.17 CID spectrum for [II-H2O]+ at $\mathrm{m} / \mathrm{z} 605.1$

Further evidence of the formation of benzaldehyde was obtained by monitoring the reaction by ${ }^{1} \mathrm{H}$ NMR. The dehydrogenation of $p$-methylbenzyl alcohol to the corresponding carboxylate using $\mathbf{1 A}$ as catalyst proceeds in 22 hours at $100{ }^{\circ} \mathrm{C}$. (Figure 2.18). The ${ }^{1} \mathrm{H}$ NMR spectrum shows the presence of $p$-methyl benzaldehyde even at short reaction times. This indicates the presence of a Ru-hydride complex analogous to III, which can dissociate the aldehyde from the metallic centre.

After the formation of III, the coordinated aldehyde undergoes a nucleophilic attack by water and a proton transfer to render the intermediate species IV. The next step comprise the release of one molecule of hydrogen and a new $\beta$-hydride elimination to give complex V. DFT studies show that both, the hydrogen release and the $\beta$-hydride elimination are energetically favoured by 12.7 and $19.7 \mathrm{Kcal} / \mathrm{mol}$ respectively. Then, the hydride complex $\mathbf{V}$ reacts with one molecule of benzyl alcohol to generate complex $\mathbf{V I}$, containing dihydrogen and alcoxide ligands. In this step the carboxylic acid coordinated to $\mathbf{V}$ is released and immediately deprotonated in the basic reaction media. The final step involves the evolution of the second molecule of hydrogen from $\mathbf{V I}$ and coordination of a water molecule to regenerate II and close the catalytic cycle. 

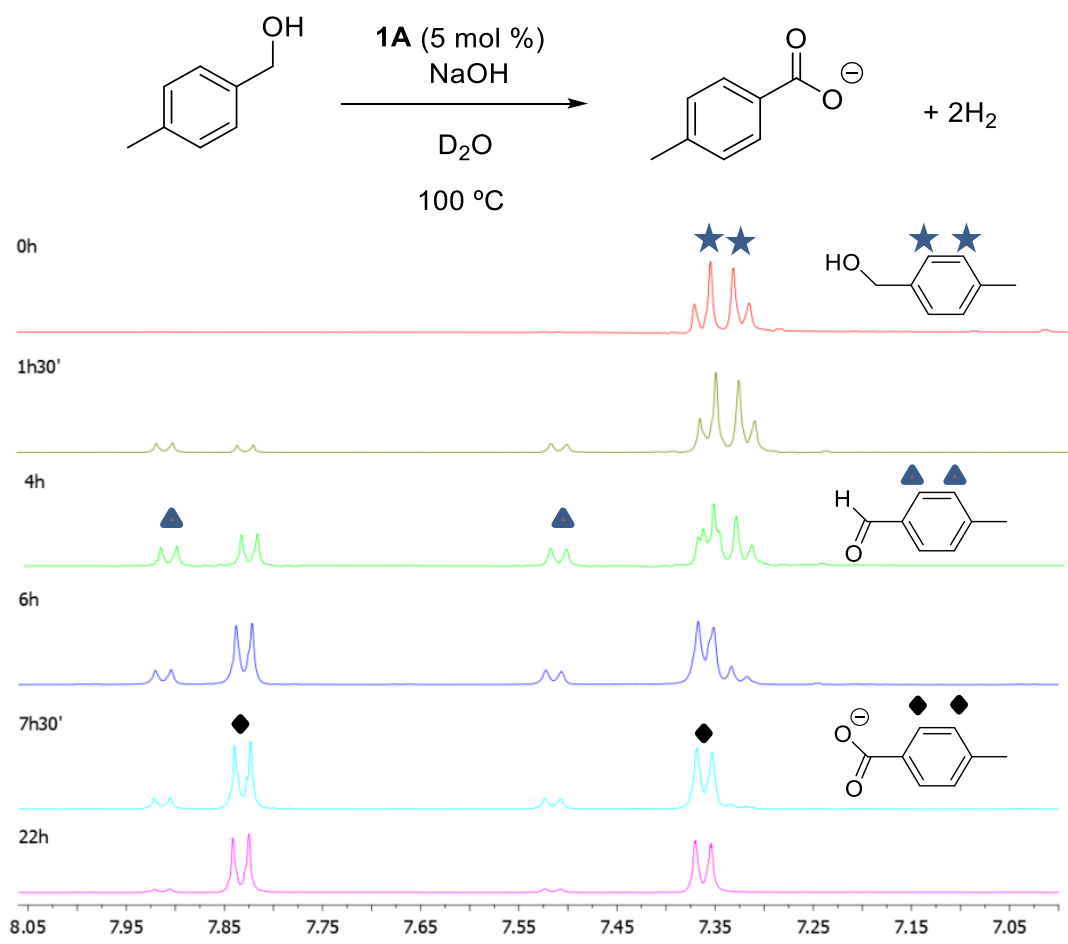

Figure 2.18 Monitoring of the dehydrogenation reaction by ${ }^{1} \mathrm{H}$ NMR

All the steps shown in Scheme 2.1 were computed. DFT Calculations were carried out in the gas phase at the M06 level of theory, and solvent corrections were subsequently performed using the SMD solvation model. The theoretical results support the proposed mechanism shown in Scheme 2.1 and match with the experimental observations obtained by NMR spectroscopy and ESI-MS.

Scheme 2.2 shows the complete energy profile for the ruthenium catalysed dehydrogenation of alcohols in aqueous media. The rate determining step of this process is the nucleophilic attack of water to the coordinated benzaldehyde, with an energy barrier of $27.5 \mathrm{Kcal} / \mathrm{mol}$. However, it should be mentioned that, as the reaction is carried out in water, the entropic factor of the calculation is overestimated, so the barrier for the real process is expected to be smaller. 


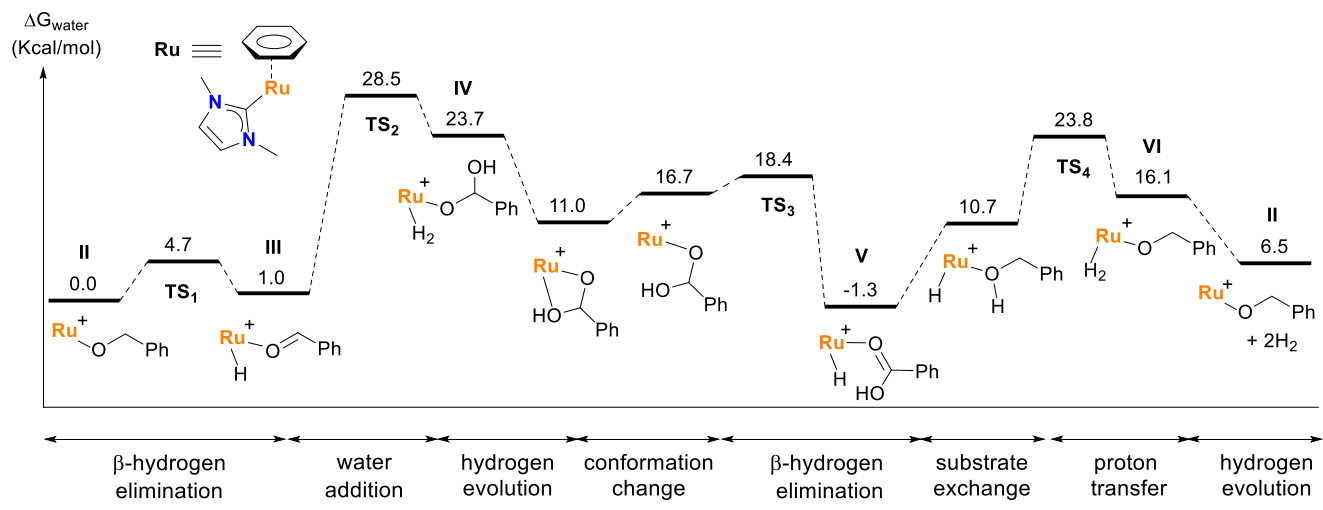

Scheme 2.2 Relative energy profile for the oxidation of benzyl alcohol to benzoic acid catalysed by $\left[\mathrm{Ru}\left(\eta^{6}-\mathrm{C}_{6} \mathrm{H}_{6}\right)(\mathrm{Cl})_{2}\left(\mathrm{NHC}-\mathrm{Me}_{2}\right)\right]$

After the nucleophilic attack, the release of the first molecule of hydrogen and the $\beta$-hydride elimination to form $\mathbf{V}$ is favoured by $25 \mathrm{Kcal} / \mathrm{mol}$. The release of hydrogen gas is in agreement with experimental observations. From a thermodynamic point of view this reaction is an entropically driven process and the driving force is the irreversible release of molecular hydrogen. To confirm this, the reaction was performed in a closed vessel and a complete inhibition was observed. In addition, the presence of hydrogen was qualitatively confirmed by gas chromatography when the reaction was set up under standard conditions.

After the formation of $\mathbf{V}, \mathbf{V I}$ is generated from a hydride-alcohol intermediate via proton transfer, with an energy barrier of $25.1 \mathrm{Kcal} / \mathrm{mol}$. Proton transfer occurs through the participation of discrete water molecules that act as proton shuttles. As for the water nucleophilic attack, it seems reasonable to consider that this barrier is lower than the computed, as the reaction is done in aqueous media. 


\subsection{CONCLUSIONS}

The preparation and immobilisation of ruthenium complexes with NHC ligands that feature different aromatic tags has been evaluated. The molecular complexes can be grafted on the surface of graphene by means of $\pi-\pi$ stacking interactions. Initial immobilisation of molecular complexes depends on the strength of $\pi$-interactions as revealed by ICP analysis. The molecular complexes functionalised with NHC ligands with a more extended aromatic system are more efficiently immobilised. The new materials show the absence of metal nanoparticles and consist of molecular complexes strongly anchored on the surface of graphene.

The catalytic properties of the molecular complexes and the new materials have been studied in the acceptorless dehydrogenation of alcohols in aqueous media. Catalytic experiments reveal that the activity of the grafted materials is higher when compared with the related homogeneous species. This enhancement of activity is attributed to an increase of molecular complex stability when anchored onto graphenic materials; which somehow mitigate deactivation pathways.

The Maitlis Hot Filtration Test shows that the catalytic hybrid materials are heterogeneous in nature when the interaction between the support and the homogeneous complex is strong. This is the case of the material based on the Ru complex with a pyrene tag $(\mathbf{3 C})$. On the other hand, the Ru complexes that form weak interactions with the support operate through a catch and release mechanism. Thus, we have observed a slight deactivation through successive runs. ICP-MS analysis show that, for these cases, metal leaching from the surface of rGO is the cause of deactivation.

An in-deep mechanistic study for the reaction has been done. NMR and ESI-MS studies show the propensity of $\left[\mathrm{Ru}(p-\mathrm{cym})\left(\mathrm{NHC}^{\mathrm{C}} \mathrm{Cl}_{2}\right]\right.$ complexes to generate empty coordination sites in aqueous media. DFT calculations support the water nucleophilic attack to a Ru-coordinated aldehyde as the rate determining step. This step has an energy barrier of $27.5 \mathrm{Kcal} / \mathrm{mol}$, which is plausible for a reaction carried out in refluxing water. 


\subsection{REFERENCES}

[1] G. Tojo, M. I. Fernández, Oxidation of Alcohols to Aldehydes and Ketones, Springer US, 2006.

[2] G. Tojo, M. I. Fernández, Oxidation of Primary Alcohols to Carboxilic Acids, Springer-Verlag Ney York, 2007.

[3] P. T. Anastas, J. Warner, Green Chemistry: Theory and Practice, Oxford University Press, Oxford, 1998.

[4] F. Cardona, C. Parmeggiani, Transition Metal Catalysis in Aerobic Alcohol Oxidation, RSC Green Chemistry Book Series, Cambidge, 2015.

[5] T. Mallat, A. Baiker, Chem. Rev. 2004, 104, 3037-3058.

[6] P. Hu, D. Milstein, Top. Organometal.Chem 2019, 63, 175-192.

[7] K. Yamaguchi, N. Mizuno, Angew. Chemie - Int. Ed. 2002, 4538-4542.

[8] S. Carrettin, P. McMorn, P. Johnston, K. Griffin, G. J. Hutchings, Chem. Commun. 2002, 7, 696-697.

[9] A. Corma, H. Garcia, Chem. Soc. Rev. 2008, 37, 2096-2126.

[10] L. Han, P. Xing, B. Jiang, Org. Lett. 2014, 16, 3428-3431.

[11] G. J. Ten Brink, I. W. C. E. Arends, R. A. Sheldon, Science 2000, 287, 16361639.

[12] J. M. Hoover, S. S. Stahl, J. Am. Chem. Soc. 2011, 133, 16901-16910.

[13] X. Jiang, J. Zhang, S. Ma, J. Am. Chem. Soc. 2016, 138, 8344-8347.

[14] R. V. Oppenauer, Recl. Trav. Chim. Pay-Bas 1937, 56, 137-144.

[15] M. Trincado, H. Grützmacher, F. Vizza, C. Bianchini, Chem. - A Eur. J. 2010, 16, 2751-2757.

[16] G. E. Dobereiner, R. H. Crabtree, Chem. Rev. 2010, 110, 681-703.

[17] S. L. Regen, G. M. Whitesides, J. Org. Chem. 1972, 37, 1832-1833.

[18] Y. Sasson, J. Blum, J. Org. Chem. 1975, 40, 1887-1896.

[19] G. Z. Wang, J. E. Bäckvall, J. Chem. Soc. Chem. Commun. 1992, 337-339. 
[20] M. L. S. Almeida, M. Beller, G. Z. Wang, J. E. Bäckvall, Chem. - A Eur. J. 1996, 2, 1533-1536.

[21] T. Zweifel, J. V. Naubron, H. Grützmacher, Angew. Chemie - Int. Ed. 2009, 48, 559-563.

[22] M. Trincado, K. Kühlein, H. Grützmacher, Chem. - A Eur. J. 2011, 17, 1190511913.

[23] S. Annen, T. Zweifel, F. Ricatto, H. Grützmacher, ChemCatChem 2010, 2, 1286-1295.

[24] H. B. Charman, Nature 1966, 278-279.

[25] H. B. Charman, J. Chem. Soc. B 1967, 629-632.

[26] H. B. Charman, J. Chem. Soc. B 1970, 584-587.

[27] A. Dobson, S. D. Robinson, Inorg. Chem. 1977, 16, 137-142.

[28] W. K. Rybak, J. J. Ziółkowski, J. Mol. Catal. 1981, 11, 365-370.

[29] M. Nielsen, A. Kammer, D. Cozzula, H. Junge, S. Gladiali, M. Beller, Angew. Chemie - Int. Ed. 2011, 50, 9593-9597.

[30] K. I. Fujita, N. Tanino, R. Yamaguchi, Org. Lett. 2007, 9, 109-111.

[31] K. I. Fujita, T. Yoshida, Y. Imori, R. Yamaguchi, Org. Lett. 2011, 13, 2278-2281.

[32] C. Gunanathan, D. Milstein, Science 2013, 341, 249-261.

[33] E. Balaraman, E. Khaskin, G. Leitus, D. Milstein, Nat. Chem. 2013, 5, 122-125.

[34] J. H. Choi, L. E. Heim, M. Ahrens, M. H. G. Prechtl, Dalton Trans. 2014, 43, 17248-17254.

[35] D. H. Nguyen, Y. Morin, L. Zhang, X. Trivelli, F. Capet, S. Paul, S. Desset, F. Dumeignil, R. M. Gauvin, ChemCatChem 2017, 9, 2652-2660.

[36] A. Sarbajna, I. Dutta, P. Daw, S. Dinda, S. M. W. Rahaman, A. Sarkar, J. K. Bera, ACS Catal. 2017, 7, 2786-2790.

[37] J. Malineni, H. Keul, M. Möller, 2015, 44, 17409-17414.

[38] Y. Sawama, K. Morita, T. Yamada, S. Nagata, Y. Yabe, Y. Monguchi, H. Sajiki, Green Chem. 2014, 16, 3439-3443. 
[39] Y. Sawama, K. Morita, S. Asai, M. Kozawa, S. Tadokoro, J. Nakajima, Y. Monguchi, H. Sajiki, Adv. Synth. Catal. 2015, 357, 1205-1210.

[40] S. McGrandle, G. C. Saunders, J. Fluor. Chem. 2005, 126, 449-453.

[41] Z. Fei, D. R. Zhu, X. Yang, L. Meng, Q. Lu, W. H. Ang, R. Scopelliti, C. G. Hartinger, P. J. Dyson, Chem. - A Eur. J. 2010, 16, 6473-6481.

[42] S. Sabater, J. A. Mata, E. Peris, ACS Catal. 2014, 4, 2038-2047.

[43] H. M. J. Wang, I. J. B. Lin, Organometallics 1998, 17, 972-975.

[44] S. Sabater, J. A. Mata, E. Peris, Organometallics 2015, 34, 1186-1190.

[45] M. K. Crawford, Y. Wang, K. B. Eisenthal, Chem. Phys. Lett. 1981, 79, 529-533.

[46] D. Tanaka, S. Horike, S. Kitagawa, M. Ohba, M. Hasegawa, Y. Ozawa, K. Toriumi, Chem. Commun. 2007, 30, 3142-3144.

[47] D. R. Dreyer, H. Jia, C. W. Bielawski, Angew. Chemie - Int. Ed. 2010, 49, 68136816.

[48] H. Jia, D. R. Dreyer, C. W. Bielawski, Tetrahedron 2011, 67, 4431-4434.

[49] J. E. Hamlin, K. Hirai, V. C. Gibson, P. M. Maitlis, J. Mol. Catal. 1982, 15, 337347.

[50] Y. Zhang, C. Chen, S. C. Ghosh, Y. Li, S. H. Hong, Organometallics 2010, 29, 1374-1378.

[51] P. Csabai, F. Joo, Organometallics 2004, 23, 5640-5643. 



\section{CHAPTER 3}

\section{Nitrile Synthesis by Amine Dehydrogenation}

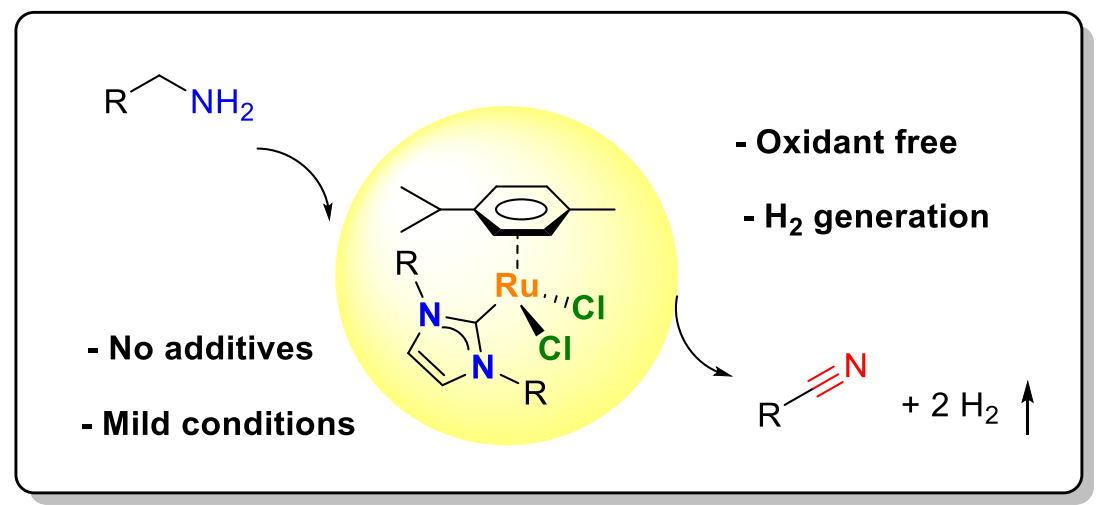





\subsection{INTRODUCTION}

Nitriles are organic molecules with one $\mathrm{C} \equiv \mathrm{N}$ bond. They are found in pharmaceuticals, herbicides, dyes or polymers, and are also regarded as valuable intermediates in organic synthesis. ${ }^{[1,2]}$

Traditional synthesis of nitriles usually involves the use of metal cyanides and substrates with suitable leaving groups. That is the case, for example, of the Sandmeyer reaction where an aromatic amine is converted into an aryl diazonium salt that then reacts with cuprous cyanide to obtain the corresponding aromatic nitrile (Figure 3.1).

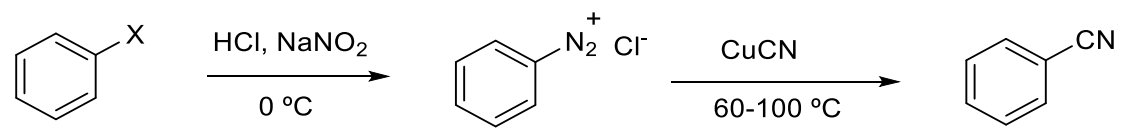

Figure 3.1 Conventional synthesis of nitriles

Although the Sandmeyer reaction dates back to 1884, it is still used at both, laboratory scale and industry. ${ }^{[3,4]}$ The sustainability of this process is questionable as the reaction is carried out under strong acidic conditions and generates stoichiometric amounts of metal waste. Then, a more sustainable approach for the synthesis of nitriles is highly desirable.

While there are numerous reports for the catalytic oxidation of primary amines to get nitriles, ${ }^{[5]}$ these systems are out of the scope of the chapter at this point. On the contrary, this section focuses on the acceptorless dehydrogenation of amines. In analogy to the $A D H$ of alcohols, nitriles can be produced from the consecutive double dehydrogenation of primary amines, generating valuable $\mathrm{H}_{2}$ as the only by-product. In addition, as the nitrile hydrogenation is a well-understood process, ${ }^{[6-9]}$ if an efficient method for the dehydrogenation of amines to nitriles with the concomitant liberation of hydrogen is developed, the amine/nitrile pair could be a promising liquid organic hydrogen carrier (LOHC). ${ }^{[10]}$ Further discussion on potential LOHC is included in chapter 4.

One of the drawbacks that needs to be tackled to develop an efficient catalyst for the dehydrogenation of amines is chemoselectivity. Due to the high reactivity of the aldimine intermediate product generated after the first dehydrogenation, secondary amines as well as imines are usually formed (Figure 3.2). ${ }^{[11]}$ 


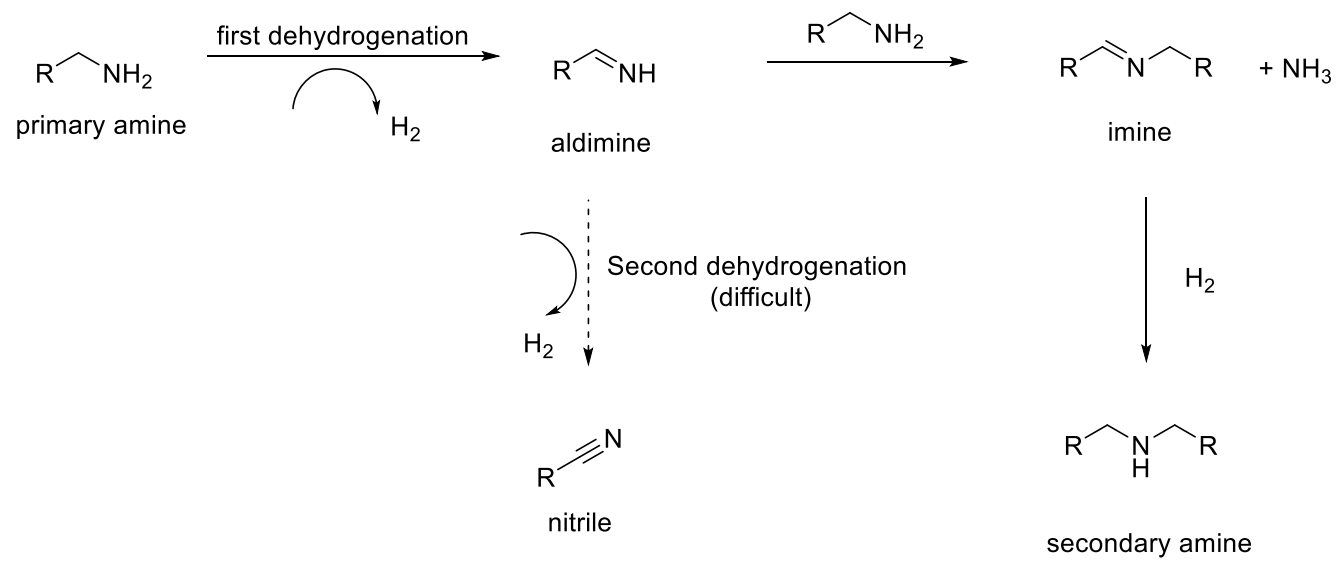

Figure 3.2 Chemoselectivity problems found in the ADH of amines

The first homogeneous catalyst capable of selectively dehydrogenate primary amines to nitriles was reported by Szymczak in 2013. ${ }^{[12]}$ The system consists of a ruthenium hydride complex with a pincer NNN ligand (I, Figure 3.3). Notably, neither secondary amines nor imines were observed at the end of the reactions. The authors attribute the excellent selectivity to the high affinity of the catalyst towards imine intermediates that allows a fast second dehydrogenation and prevents the release of the aldimine product, which may enter in the cycle for transamination reactions. ${ }^{[13]}$ Few years later, Prof. W. Leitner and coworkers developed another Ru based catalyst that is able to dehydrogenate a wider scope of primary amines to nitriles at a slightly lower temperature $\left(70^{\circ} \mathrm{C}\right.$ vs $110^{\circ} \mathrm{C}$ ) with the concomitant release of 2 equivalents of hydrogen gas (Figure 3.3, bottom). ${ }^{[14]}$ However, the addition of $10 \mathrm{~mol} \%$ of a strong base (tBuOK) was necessary, which is a drawback for large-scale applications. 


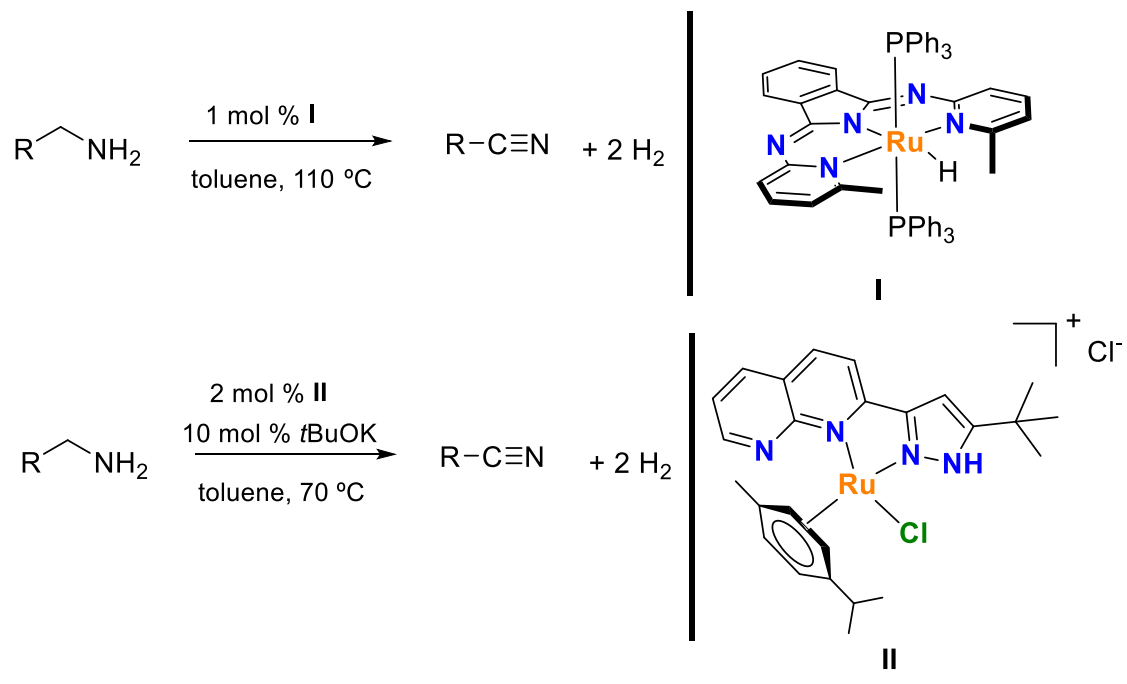

Figure 3.3 Homogeneous systems reported by Szymczak (top) and Leitner (down) for the ADH of primary amines to synthesize nitriles

Regarding heterogeneous catalysts, the dehydrogenation of amines have been reported using systems based on iridium, palladium, or ruthenium. However, these systems show some limitations as a lack of selectivity, ${ }^{[15]}$ stops after the first dehydrogenation, ${ }^{[16]}$ or need the presence of oxygen, ${ }^{[15,17,18]}$ avoiding the generation of hydrogen. Yet, there is one recent report that allows the direct generation of nitriles and hydrogen from primary amines based on copper nanoparticles on an aluminium hydrotalcite support (Figure 3.4). ${ }^{[19]}$ This system is able to dehydrogenate aliphatic and benzylic amines under a relatively low catalyst loading ( $3 \mathrm{~mol} \%$ ) and moderate temperature $\left(120^{\circ} \mathrm{C}\right)$ in DMF. In addition, the system can be recycled for five consecutive runs without any observed decrease in activity.

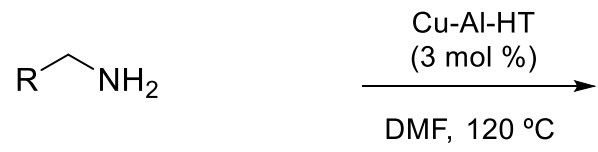

alkilyc and benzyilic amines
5 cycles of catalyst recycling

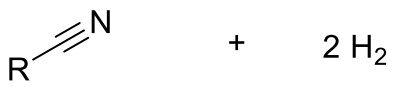

$99 \%$ yield

Figure 3.4 Dehydrogenative oxidation of amines catalysed by a recyclable Cu catalyst 


\section{Chapter 3}

Taking all this into account, the objective of this chapter is to study the activity of complexes of type $\left[\mathrm{Ru}(\mathrm{p}-\mathrm{cym})(\mathrm{NHC}) \mathrm{Cl}_{2}\right]$ in the $\mathrm{ADH}$ of amines. To check the influence of modifying the structure of NHC the ligand, three different ruthenium-based compounds that feature NHCs with different electronic properties were prepared (Figure 3.5).

The three complexes were tested in the dehydrogenation of benzylic and aliphatic amines. In addition, the hybrid material 3C-rGO was studied as a potential recyclable catalyst for the ADH of primary amines.

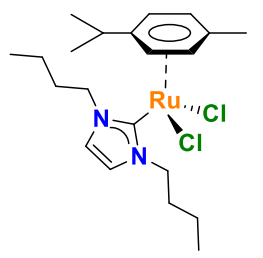

4D

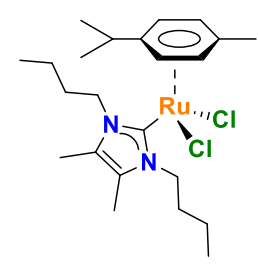

$5 E$

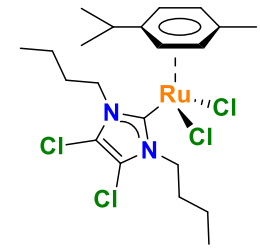

6F

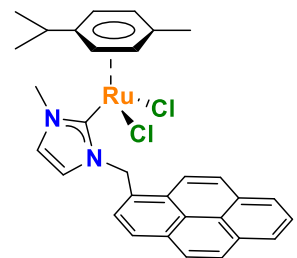

$3 \mathrm{C}$

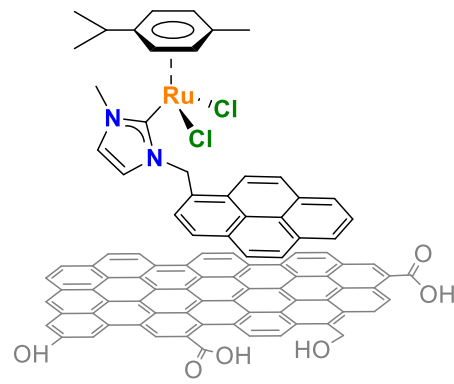

3C-rGO

Figure 3.5 Ru complexes tested in the ADH of primary amines 


\subsection{RESULTS AND DISCUSSION}

\subsubsection{Synthesis of ruthenium complexes}

In order to analyse the influence of electronic properties of the catalyst in the dehydrogenation of amines, three different complexes with the general formula $\left[\mathrm{Ru}(p-\mathrm{cym})(\mathrm{NHC}) \mathrm{Cl}_{2}\right]$ were synthesised.

Compounds $4 \mathrm{D}, \mathbf{5 E}$ and $\mathbf{6 F}$ differ in the NHC ligand, which in all cases has two $n$-butyl substituents at the nitrogen atoms but different groups $(\mathrm{H}, \mathrm{Me}$ or $\mathrm{Cl})$ at both positions of the backbone.
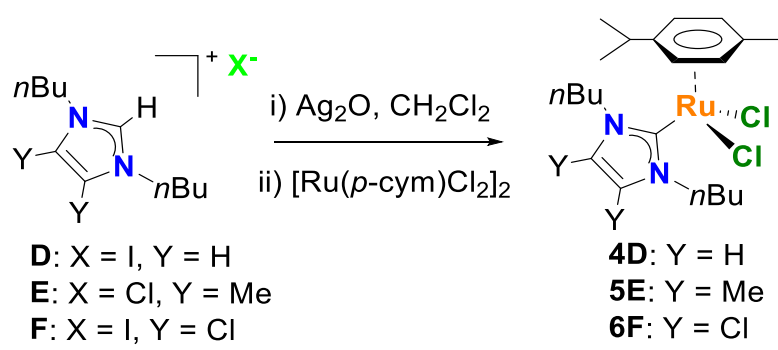

Figure 3.6 Synthesis of Ru complexes 4D, 5E and 6F

The new complexes $\mathbf{5 E}$ and $\mathbf{6 F}$ were obtained by the in situ formation of the silver carbene intermediate which was then reacted with $\left[\mathrm{Ru}(p-c y m) \mathrm{Cl}_{2}\right]_{2}$, affording the desired ruthenium complex in moderate yield after purification by flash chromatography.

The new complexes were fully characterised by ${ }^{1} \mathrm{H} N M R,{ }^{13} \mathrm{C} N M R$, elemental analysis, ESI-MS and HRMS (all the details are included in chapter 6 ).

\subsubsection{Catalytic dehydrogenation of primary amines}

Complexes 4D, 5E and 6F were initially tested in the dehydrogenation of benzyl amine as model substrate. To set up the reactions one equivalent of amine and $2 \mathrm{~mol} \%$ of catalyst were dissolved in $3 \mathrm{~mL}$ of solvent and heated under vigorous reflux in an open system. The influence of solvent, additives and reaction atmosphere $\left(\mathrm{O}_{2}\right.$ or $\left.\mathrm{N}_{2}\right)$ was studied, and the results are summarised in Table 3.1. As mentioned in section 3.1, one of the major challenges that needs to be addressed in the selective dehydrogenation of amines to nitriles is product selectivity. At the end of the reaction different products may be present due to transamination reactions. In this case, the generation of nitrile was always observed along with the formation of the corresponding imine as the only by-product. 
Table 3.1 Optimization of reaction conditions for the dehydrogenation of primary amines<smiles>[R]c1ccc(CN)cc1</smiles><smiles>[R]c1ccc(C=NCc2ccc([R])cc2)cc1</smiles>

\begin{tabular}{|c|c|c|c|c|c|c|c|}
\hline \multirow[t]{2}{*}{ Entry } & \multirow[t]{2}{*}{ Catalyst } & \multirow[t]{2}{*}{$\mathbf{R}$} & \multirow[t]{2}{*}{ Additive } & \multirow[t]{2}{*}{ Solvent } & \multirow[t]{2}{*}{ Conversion $(\%)^{a}$} & \multicolumn{2}{|c|}{ Selectivity (\%) } \\
\hline & & & & & & Nitrile & Imine \\
\hline 1 & - & $\mathrm{H}$ & - & Toluene & 0 & - & - \\
\hline 2 & 4D & $\mathrm{H}$ & AgOTf & Toluene & 92 & 49 & 51 \\
\hline 3 & 4D & $\mathrm{H}$ & tBuOK & Toluene & 34 & 68 & 32 \\
\hline 4 & 4D & $\mathrm{H}$ & $\mathrm{Cs}_{2} \mathrm{CO}_{3}$ & Toluene & 88 & 70 & 30 \\
\hline 5 & 4D & $\mathrm{H}$ & $\mathrm{NaOAc}$ & Toluene & 97 & 60 & 40 \\
\hline 6 & 4D & $\mathrm{H}$ & - & Toluene & 98 & 74 & 26 \\
\hline 7 & $5 E$ & $\mathrm{H}$ & - & Toluene & 95 & 73 & 27 \\
\hline 8 & $6 F$ & $\mathrm{H}$ & - & Toluene & 100 & 75 & 25 \\
\hline 9 & $6 F$ & $\mathrm{CH}_{3}$ & - & Toluene & 97 & 74 & 26 \\
\hline 10 & $6 F$ & $\mathrm{CH}_{3}$ & - & $\mathrm{MeCN}$ & 24 & 50 & 50 \\
\hline 11 & $6 F$ & $\mathrm{CH}_{3}$ & - & ¡PrOH & 54 & 68 & 32 \\
\hline 12 & $6 F$ & $\mathrm{CH}_{3}$ & - & $\mathrm{tBuOH}$ & 75 & 65 & 35 \\
\hline 13 & $6 F$ & $\mathrm{CH}_{3}$ & - & $\mathrm{nBuOH}$ & 39 & 64 & 36 \\
\hline 14 & $6 F$ & $\mathrm{H}$ & $\mathrm{N}_{2}$ (bubbling) & Toluene & 98 & 71 & 29 \\
\hline 15 & $6 F$ & $\mathrm{H}$ & $\mathrm{O}_{2}(1 \mathrm{~atm})$ & Toluene & $<5$ & n. d. & n. d. \\
\hline
\end{tabular}

Reaction conditions: Substrate $(0.3 \mathrm{mmol})$, catalyst $(2 \mathrm{~mol} \%), 3 \mathrm{~mL}$ of solvent at $110^{\circ} \mathrm{C}$ for $8 \mathrm{~h}$. [a] Conversion determined by GC analysis using anisole as the internal standard. (n. d.; not determined). Selectivities determined by ${ }^{1} \mathrm{H}$ NMR.

A control experiment revealed that the dehydrogenation of benzyl amine does not take place in the absence of catalyst (entry 1 ). In the presence of halide abstractor (silver triflate, AgOTf), the reaction with 4D as catalyst afforded a $92 \%$ conversion but with a complete lack of selectivity (entry 2). Replacement of a halide abstractor by a base gave different results. In the case of a strong base such as potassium tert-butoxide (entry 3), the conversion decreased to $34 \%$, albeit with a slight increase in selectivity (68/32 towards nitrile). As the strength of the base decreases, the conversion increases with similar selectivity. The best result with 4D as catalyst was obtained in the absence of any additive, affording full conversion in eight hours with a moderate selectivity of 74/26 (compare entries 3, 4, 5 and 6). Complexes $5 \mathbf{E}$ and $6 \mathrm{~F}$ with different electronic properties showed no substantial difference in terms of activity or selectivity to that obtained with 4D (entries 6-8). If the reaction of benzyl amine catalysed by $4 \mathrm{D}, 5 \mathrm{E}$ or $\mathbf{6 F}$ is carried out in a closed reactor, the only product observed is the related imine. ${ }^{[20]}$ The dehydrogenative oxidation of amines 
to obtain nitriles is a non-spontaneous process. Removal of hydrogen gas from the reaction media entropically favours the formation of nitrile and drives the reaction forward. Hence, a vigorous solvent reflux was needed to facilitate hydrogen release.

As slightly better results were obtained with $\mathbf{6 F}$ (entry 8 ), this complex was used for the subsequent experiments. The reaction is highly dependent on the solvent used. When acetonitrile was the solvent of choice, a discrete $24 \%$ conversion was obtained (entry 10). Alcohol-type solvents neither improve the catalytic results, giving always lower conversion and selectivity (entries 11-13). To rule out the oxidation of amine by molecular oxygen instead of the dehydrogenation pathway two different experiments were done. First, the reaction was carried out under a $\mathrm{N}_{2}$ atmosphere, with similar results (compare entries 8 and 14).This fact suggests that oxygen is not needed in the reaction media. In fact, the presence of molecular oxygen (1 bar) in the reaction media was detrimental and a low conversion (<5\%) was achieved (entry 15). These two experiments suggest that oxygen is not the final oxidant.

The scope of the reaction and its limitations were evaluated under the optimal conditions (Table 3.2). The reactions were carried out dissolving $0.3 \mathrm{mmol}$ of substrate and heating in refluxing toluene $(3 \mathrm{~mL})$ for 8 hours using a catalyst loading of $2 \mathrm{~mol} \%$. For this study complex $\mathbf{3 C}$ was also included in view of, in a next step, develop a recyclable catalyst (3C-rGO) for the dehydrogenation of amines. 
Table 3.2 Reaction scope for the dehydrogenation of amines

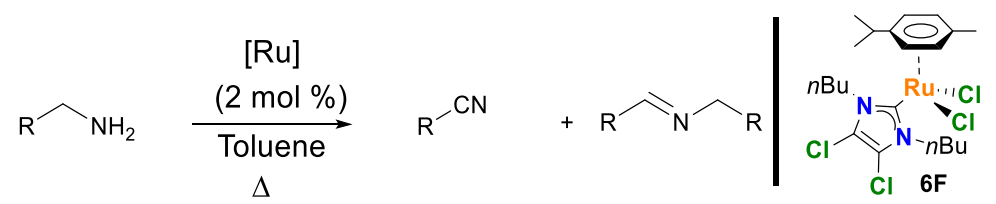

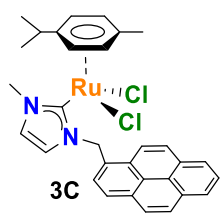

\begin{tabular}{cccccc}
\hline Entry & Catalyst & $\mathbf{R}$ & Conversion (\%) $^{\mathbf{a}}$ & \multicolumn{2}{c}{ Selectivity (\%) } \\
\cline { 4 - 6 } & & & & Nitrile & Imine \\
\hline 1 & 6F & $\mathrm{Ph}$ & 95 & 75 & 25 \\
2 & 3C & $\mathrm{Ph}$ & 99 & 82 & 18 \\
3 & 3C-rGo & $\mathrm{Ph}$ & $100^{\mathrm{b}}$ & 65 & 35 \\
\hline 4 & $\mathbf{6 F}$ & $(4-\mathrm{Cl}) \mathrm{Ph}$ & 95 & 50 & 50 \\
5 & 3C & (4-Cl)Ph & 83 & 57 & 43 \\
\hline 6 & 6F & (4-Me)Ph & 97 & 74 & 26 \\
7 & 3C & (4-Me)Ph & 93 & 70 & 30 \\
\hline 8 & 6F & (4-OMe)Ph & 99 & 69 & 31 \\
9 & 3C & (4-OMe)Ph & 97 & 68 & 32 \\
\hline 10 & 6F & (4-CF $) \mathrm{Ph}$ & 95 & 62 & 38 \\
11 & 3C & (4-CF $) \mathrm{Ph}$ & 78 & 52 & 48 \\
\hline 12 & 6F & 1-Naphtyl & 80 & 55 & 45 \\
13 & 3C & 1-Naphtyl & 82 & 57 & 43 \\
\hline
\end{tabular}

Reaction conditions: Substrate $(0.3 \mathrm{mmol})$, catalyst $(2 \mathrm{~mol} \%), 3 \mathrm{~mL}$ of toluene at $110{ }^{\circ} \mathrm{C}$ for $8 \mathrm{~h}$. [a] Conversion determined by GC analysis using anisole as the internal standard. Selectivities determined by ${ }^{1} \mathrm{H}$ NMR [b] After $6 \mathrm{~h}$ of reaction.

Both $3 \mathrm{C}$ and $6 \mathrm{~F}$ are competent catalysts for the dehydrogenation of substituted benzyl amines, giving excellent activities. Only in the case of bulky amines such as 1-naphtylmethylamine lower conversions (ca. $80 \%$, entries 12 and 13) were obtained. The catalytic activity of $\mathbf{3 C}$ and $\mathbf{6 F}$ is similar (Figure 6.32 in experimental section). In general, the reaction proceeds with low to moderate selectivity. The best result in terms of selectivity is obtained with catalyst $\mathbf{3 C}$ using benzyl amine (entry 2). Ligand modification does not lead to any significant difference and more efforts to understand the factors that govern the selectivity are needed. These results are in agreement with that recently reported by Bellemin-Laponnaz and co-workers using complexes of type $\left[\mathrm{Ru}(p\right.$-cym $)\left(\mathrm{NHC}^{-} \mathrm{Cl}_{2}\right]$ with thioetherfunctionalised NHC ligands. ${ }^{[21]}$

The hybrid material $\mathbf{3 C - r G O}$ is a competent catalyst in the dehydrogenation of primary amines (Table 3.2, entry 3). In fact, the hybrid material is even more active and the grafting process increases the activity of the molecular compound. 
The reaction time is shortened by 2 hours under the same reaction conditions using the same metal loading. This result shows that anchoring complex $\mathbf{3 C}$ by non-covalent interactions has a catalytic benefit, most probably due to an increase in the stability of active species.

Then, the reaction scope using primary lineal alkyl amines was also investigated (Table 3.3):

Table 3.3 Reaction scope and limitations in the dehydrogenation of primary alkyl amines

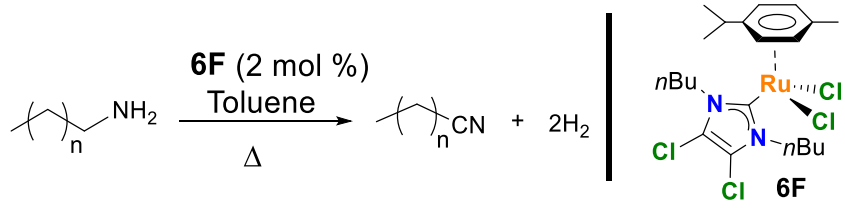

\begin{tabular}{cccccc}
\hline Entry & Amine & $\mathbf{n}$ & Conversion (\%) $^{\mathbf{a}}$ & \multicolumn{2}{c}{ Selectivity (\%) } \\
\cline { 5 - 6 } & & 5 & & Nitrile & Imine \\
\hline 1 & Heptylamine & 55 & 78 & 22 \\
2 & Octylamine & 6 & 99 & 89 & 11 \\
3 & Dodecylamine & 10 & $100(97)$ & 100 & 0 \\
4 & Tetradecylamine & 12 & $95(90)$ & 100 & 0 \\
5 & Hexadecylamine & 14 & $97(92)$ & 100 & 0 \\
6 & Octadecylamine & 16 & $95(91)$ & 100 & 0 \\
\hline
\end{tabular}

Reaction conditions: Substrate $(0.3 \mathrm{mmol})$, catalyst $(2 \mathrm{~mol} \%), 3 \mathrm{~mL}$ of toluene at $110{ }^{\circ} \mathrm{C}$ for $24 \mathrm{~h}$. [a] Conversion determined by GC analysis using anisole as the internal standard. Selectivities determined by ${ }^{1} \mathrm{H}$ NMR.

Complex $6 \mathrm{~F}$ is able to dehydrogenate primary linear alkyl amines under the same reaction conditions used for the dehydrogenation of benzyl amines. Better results in terms of selectivity towards the formation of nitrile were obtained in the case of aliphatic amines. Increasing the number of carbons in the aliphatic chain blocks the formation of the imine product. For instance, with heptyl and octyl amines moderate to good selectivity is observed (Table 3.3, entries 1 and 2). However, when the chain becomes longer excellent results are obtained as complete selectivity towards nitrile is observed in a $100 \%$ conversion (entries 3 to 6 ). Selectivity control using long-chain alkyl amines or azides has been previously reported by the groups of prof. H. García, Dr. S. Bellemin-Laponnaz and Dr. S. Díez-González. ${ }^{[21-23]}$ 


\section{Chapter 3}

\section{Recycling studies}

Given the high activity of $\mathbf{3 C - r G O}$ in the dehydrogenation of amines, its reusability was studied. Recycling experiments were carried out in a $0.3 \mathrm{mmol}$ scale using benzyl amine as model substrate, at a catalyst loading of $2 \mathrm{~mol} \%$ (based on Ru). At selected times, aliquots were taken and analysed by GC, until a max conversion of $50 \%$ was reached. After that, the catalyst was washed thoroughly with toluene and reused in the following run.

The catalyst performs the reaction for eight times without suffering from any deactivation. Then, in run 9 there is a slight decrease in activity that is maintained in run 10 (Figure 3.7). The results show that the immobilisation of the molecular species onto graphene results in a highly recyclable system.
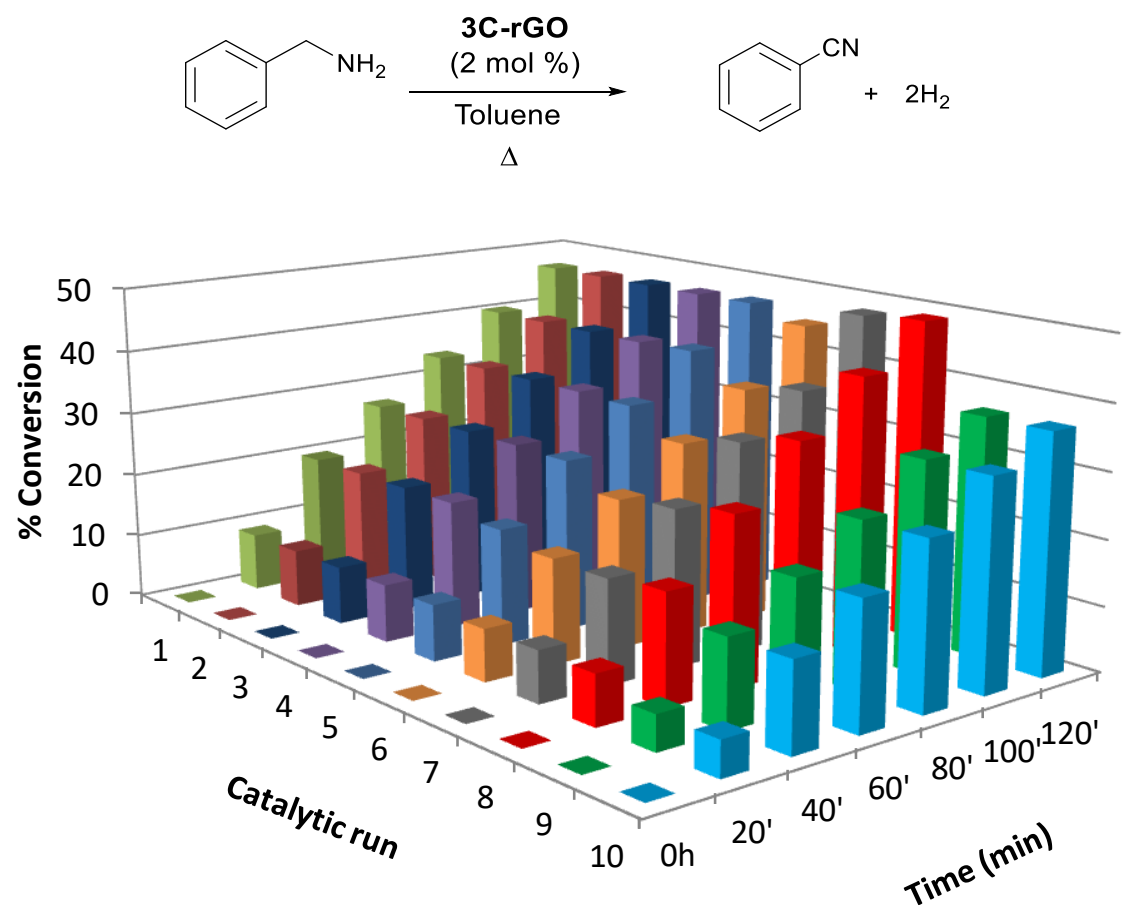

Figure 3.7 Recycling experiments on the dehydrogenation of benzyl amine using the hybrid material 3C-rGO. Reaction profile for each run determined by GC analysis up to a maximum of ca. $50 \%$ conversion 
To rule out the presence of homogeneous species during the catalytic reaction, the hot filtration test was carried out under the same reaction conditions used for the recycling studies. After 2 hours of reaction (conversion $50 \%$ ), the solid catalyst was removed by filtration "in hot", and the filtrate was stirred for 2 hours at $110{ }^{\circ} \mathrm{C}$. Analysis by GC showed no further conversion. In a parallel experiment, the hybrid material 3C-rGO previously removed was treated with benzyl amine and toluene and stirred for 2 hours, reaching a $48 \%$ conversion. This experiment shows that $3 \mathrm{C}$ is strongly anchored onto $\mathrm{rGO}$ by non-covalent interactions and that the catalysis takes place in a heterogeneous manner. In other words, the molecular species are not detached from the surface of graphene under the reaction conditions.

During the recycling experiment, the metal content in the reaction media was analysed by ICP-MS after each run. According to the low deactivation of the catalyst, the metal content found in the filtrates for each run was negligible $(<5 \mathrm{ppb})$. Immobilizing Ru complexes onto $\mathrm{rGO}$ gives rise to a recyclable material that affords the dehydrogenation of benzyl amines with low metal contamination in the final product.

The morphology of $\mathbf{3 C}$-rGO after the tenth catalytic run was studied by TEM (Figure 3.8). After the catalysis the graphene preserves its 2D nature, with a slight increase in the number of wrinkles. No metal nanoparticles have been formed and the EDS spectrum shows the presence of ruthenium, indicating the molecular nature of the catalytic active species.

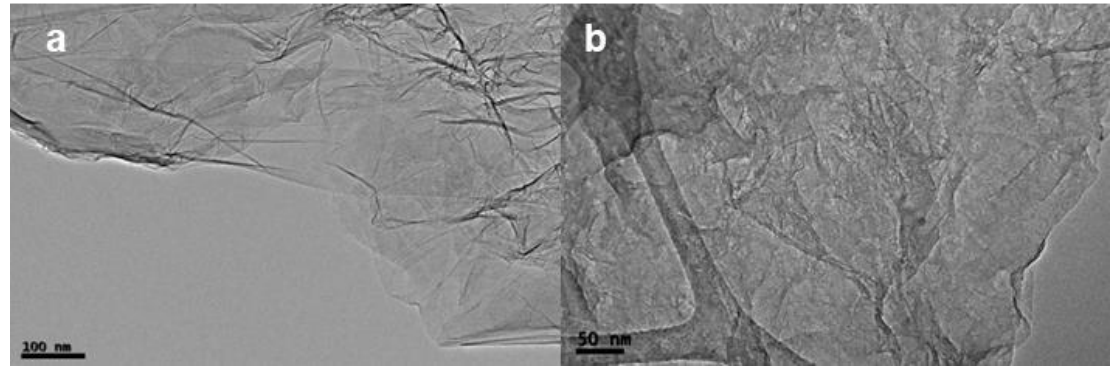

Figure 3.8 HRTEM images of 3C-rGO before (a) and after (b) ten catalytic runs 


\section{Mechanistic considerations}

Preliminary mechanistic studies were performed by means of NMR and ESI-MS. First, a reaction profile for the dehydrogenation of $p$-methoxy benzyl amine mediated by complex $6 \mathrm{~F}$ was obtained by ${ }^{1} \mathrm{H}$ NMR monitoring of the reaction in an NMR tube, using $d^{8}$-toluene as solvent (Figure 3.9).

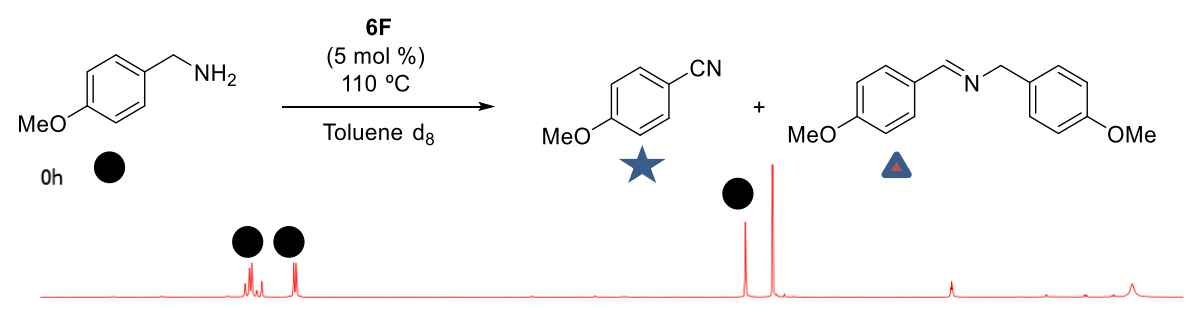

$2 \mathrm{~h}$

$5 h$

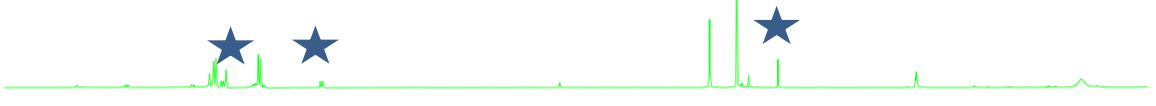

$\hat{\imath}$

$20 \mathrm{~h}$
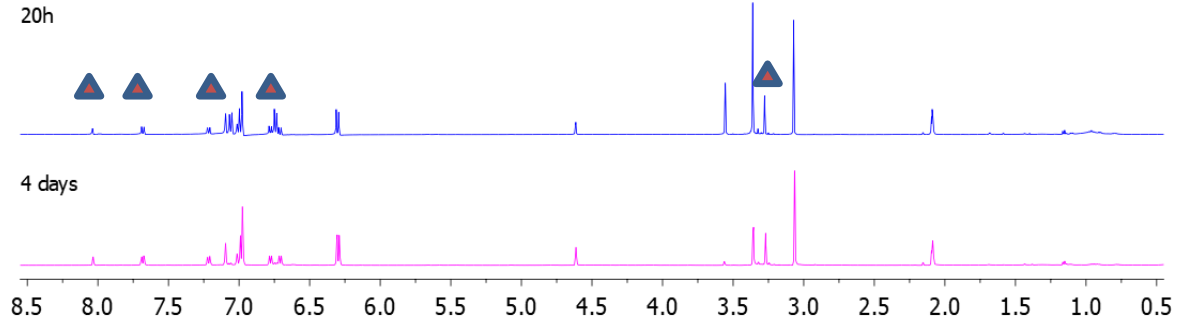

Figure 3.9 ${ }^{1} \mathrm{H}$ NMR reaction monitoring for the dehydrogenation of $p$-methoxy benzyl amine showing the formation of nitrile and imine products

The ${ }^{1} \mathrm{H}$ NMR spectra in Figure 3.9 show that nitrile is the first product formed (appears after two hours of reaction). However, the reaction kinetics in the NMR tube is not fast, and the reaction takes four days to achieve full conversion. This difference in kinetics between this experiment and the standard conditions is assigned to the absence of an efficient reflux in the NMR tube. Hydrogen release from the reaction media is the driving force that shifts the equilibrium. In an NMR tube there is not an intense solvent bubbling that facilitates gas evolution and thus the reaction is slowed down. Importantly, no amines coming from transamination 
processes were observed, and at the end of the reaction the only products were the nitrile and the imine.

Although in principle the presence of dissolved hydrogen can be identified in the ${ }^{1} \mathrm{H}$ NMR spectrum, in this case it was not observed, most probably because the gas needs to be evolved to drive the reaction forward. As an alternative, a different experiment was designed to qualitatively identify the formation of hydrogen. $A$ standard reaction was set-up using benzyl amine as substrate and complex $6 \mathrm{~F}$ as catalyst. After two hours, a sample of the generated gas was injected into a GC using a gas-syringe. The mass spectrum shows the presence of hydrogen gas, confirming the dehydrogenation pathway for the generation of nitriles.

In an attempt to study the reaction mechanism and identify any reaction intermediate, ESI-MS experiments were performed. A solution of complex $6 \mathrm{~F}$ and $p$-methyl benzyl amine was heated in toluene at $70{ }^{\circ} \mathrm{C}$ for 10 minutes and the reaction mixture was analysed by direct injection into ESI-MS (Figure 3.10).

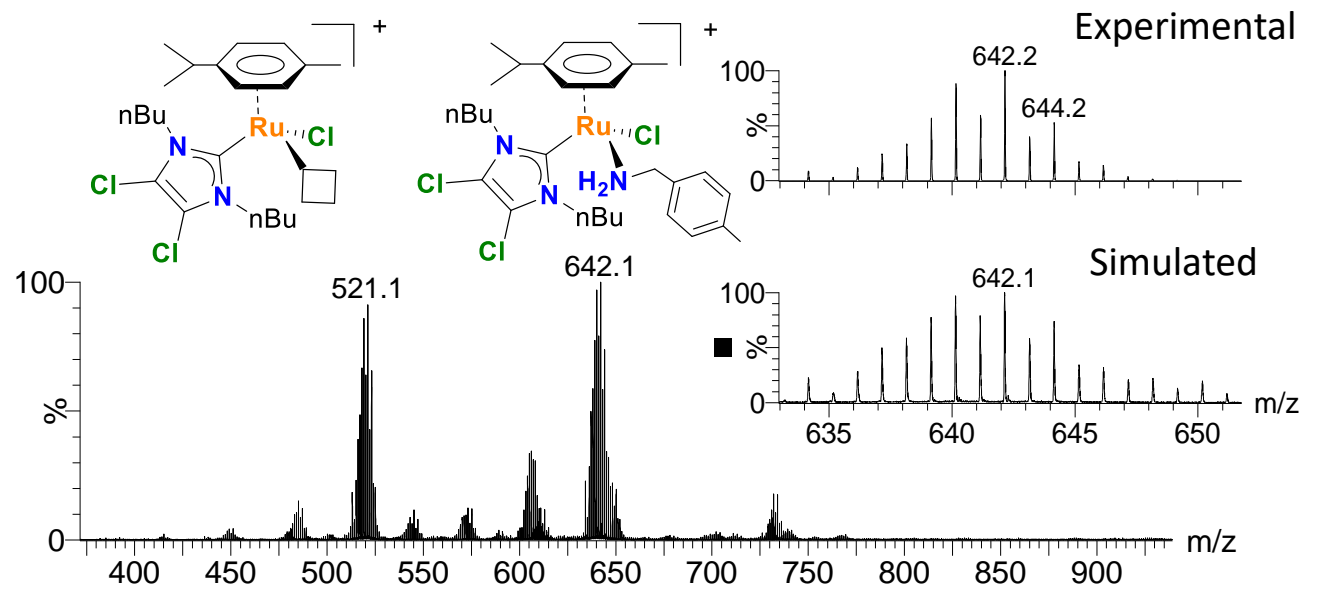

Figure 3.10 ESI-MS spectrum of complex $6 \mathrm{~F}$ in the presence of $p$-methyl benzyl amine under stoichiometric conditions. Schematic drawings of the identified species: i) with a vacant coordination site $[\mathrm{M}-\mathrm{Cl}]^{+}$and ii) with a coordinated $p$-methyl benzyl amine $\left[\mathrm{M}-\mathrm{Cl}+\mathrm{H}_{2} \mathrm{NCH}_{2} \mathrm{PhMe}\right]^{+}$

The MS spectrum shows the presence of two predominant species in solution. The peak at $\mathrm{m} / \mathrm{z}=521.1$ is attributed to complex $6 \mathrm{~F}$ with one vacant site coming from the dissociation of one chloride ligand. This behaviour corresponds with that observed for complex $\mathbf{1 A}$ in water media. The second major peak appears at $\mathrm{m} / \mathrm{z}=642.1$ and is due to the formation of complex $6 \mathrm{~F}$ with one coordinated 
$p$-methyl benzyl amine. Generation of cationic complexes by replacement of a halide ligand for an $\mathrm{L}$ type ligand has been previously reported for $\left[\mathrm{Ru}(p-\mathrm{cym})(\mathrm{NHC}) \mathrm{Cl}_{2}\right]$ complexes. ${ }^{[24]}$ The molecular composition of this peak was unambiguously confirmed by comparing the experimental and the simulated isotopic pattern distribution, which show a perfect match. When this experiment was performed at $110{ }^{\circ} \mathrm{C}$ none of this species could be detected by ESI-MS, suggesting that:

- The coordination of benzyl amine is observed at low temperatures

- A raise in temperature overcomes the activation energy needed for the transformation of amine into nitrile.

To confirm that a complex with the molecular formula $\left[\mathrm{M}-\mathrm{Cl}+\mathrm{H}_{2} \mathrm{NCH}_{2} \mathrm{PhMe}\right]^{+}$is a plausible reaction intermediate and discard that it is only formed in the ESI-MS we set-up the synthesis of the ruthenium complex containing a coordinated benzyl amine. Using a similar methodology as reported by Saunders and co-workers, ${ }^{[25]}$ complex 7G was reacted with $p$-methyl benzyl amine in a DCM/MeOH mixture, at room temperature and in the presence of sodium tetrafluoroborate as halide abstractor (Figure 3.11). After 10 minutes, the reaction afforded complex $\mathbf{7 G}^{\prime}$ in quantitative yield. The new compound was characterised by ${ }^{1} \mathrm{H} N M R,{ }^{13} \mathrm{C} N M R$ and X-Ray diffraction.

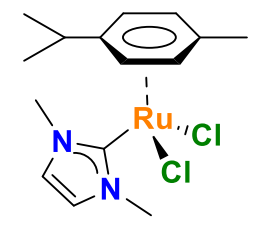

$7 G$

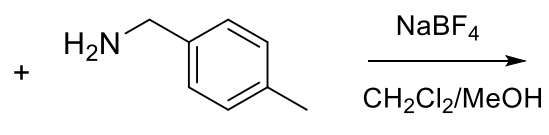

Figure 3.11 Synthesis of 7G'

The first evidence that replacement of a chloride ligand for a benzyl amine has taken place is the appearance in the ${ }^{1} \mathrm{H}$ NMR spectrum of 4 doublets instead of two for the $p$-cymene ligand in the range between 5.8 and $5.4 \mathrm{ppm}$ (Figure 3.12). The appearance of two signals (shifted at 4 and $3.8 \mathrm{ppm}$ ) instead of one for the methyl groups at the $\mathrm{N}$-substituents of the $\mathrm{NHC}$ further indicate the amine coordination. In general the number and multiplicity of signals in Figure 3.12 is consistent with the symmetry of a piano-stool complex with three different coordinated ligands. 


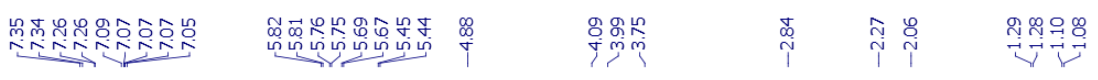

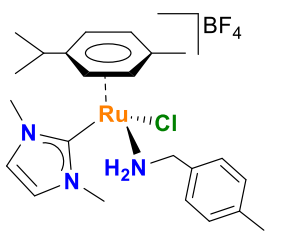

7G'

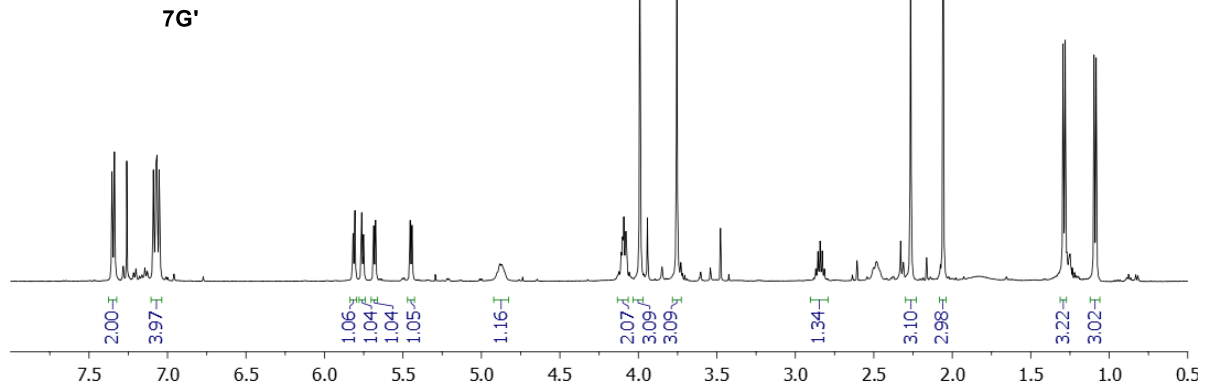

Figure 3.12 ${ }^{1} \mathrm{H}$ NMR spectrum of compound 7G' in $\mathrm{CDCl}_{3}$

Crystals of 7G'were obtained by slow diffusion of hexane in a concentrated solution of the compound in chloroform.

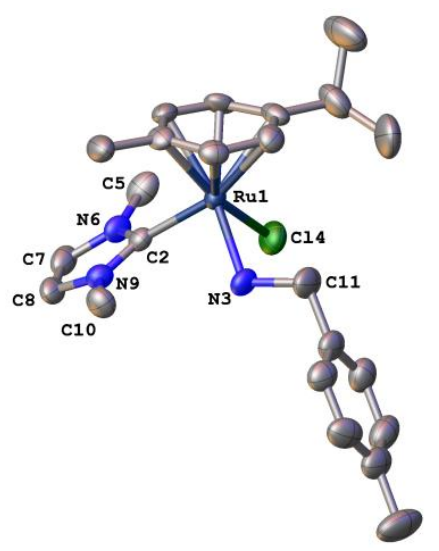

Figure 3.13 Molecular diagram of 7G'. Ellipsoids are shown at $50 \%$ of probability level. All hydrogen atoms and counterion $\left(\mathrm{BF}_{4}{ }^{-}\right)$have been ommited for clarity

The molecular structure of $\mathbf{7 G}^{\mathbf{\prime}}$ (Figure 3.13) confirms the presence of a coordinated $p$-methyl benzyl amine. As stated, the structure can be regarded as a three legged piano-stool. Along with the NHC ligand, one chloride, the benzyl amine and the 
p-cymene complete the coordination sphere of the ruthenium cationic compound. Table 3.4 summarises the most representative bond lengths and angles. The $\mathrm{Ru}-\mathrm{C}_{\text {carbene }}$ distance and angles around the ruthenium sphere lie in the expected range when compared with similar Ru-Carbene complexes. ${ }^{[26]}$

Table 3.4 Selected bond lengths $(\AA)$ and angles $\left(^{\circ}\right)$ of complex 7G'

\begin{tabular}{|l|cccc}
\cline { 2 - 5 } \multicolumn{1}{c|}{} & Bonds & $(\mathbf{A})$ & Angles & () \\
\hline \multirow{3}{*}{ 7G' $^{*}$} & $\mathrm{Ru}(1)-\mathrm{C}(2)$ & $2,074(3)$ & $\mathrm{C}(2)-\mathrm{Ru}(1)-\mathrm{N}(3)$ & $90.3(3)$ \\
& $\mathrm{Ru}(1)-\mathrm{Cl}(4)$ & $2,401(2)$ & $\mathrm{C}(2)-\mathrm{Ru}(1)-\mathrm{Cl}(4)$ & $87,2(2)$ \\
& $\mathrm{Ru}(1)-\mathrm{N}(3)$ & $2,144(7)$ & $\mathrm{N}(3)-\mathrm{Ru}(1)-\mathrm{Cl}(4)$ & $79,6(2)$ \\
& $\mathrm{Ru}(1)-\mathrm{C}_{\text {centroid }}$ & 1.708 & & \\
& & & & \\
\hline
\end{tabular}

A tentative mechanism is proposed based on experimental evidences (Scheme 3.1). Starting from complex 7G, reaction with one molecule of benzyl amine affords the fully characterised complex $\mathbf{7 G}^{\prime}$. This compound is a potential intermediate and a similar complex of formula $\left[\mathrm{Ru}(p\right.$-cym $\left.)\left(\mathrm{H}_{2} \mathrm{NCH}_{2} \mathrm{Ph}\right) \mathrm{Cl}_{2}\right]$ has been recently proposed by Bellemin-Laponnaz and co-workers as a transient species for the ADH of primary amines catalysed by $\left[\mathrm{Ru}(p-c y m) \mathrm{Cl}_{2}\right]_{2} \cdot{ }^{[27]}$ Reaction of $\mathbf{7 \mathbf { G } ^ { \prime }}$ with a second molecule of benzyl amine, generates compound I and starts the cycle. Subsequently, a $\beta$-hydride elimination forms the aldimine-coordinated complex II. This transformation has been proposed for the acceptorless dehydrogenative coupling of amines (transamination) and a similar species has been detected by ESI-MS in the dehydrogenative coupling of alcohols. ${ }^{[28]}$

The stability and reactivity of complex II are key for the final selectivity. If II reacts with a new amine the imine by-product is formed. On the contrary, if the coordinated aldimine suffers a fast $\alpha$-hydride elimination a dihydrogen coordinated intermediate is formed which, after hydrogen evolution, generates III. Then, a second $\beta$-hydride elimination generates the hydride-species IV. The proposal of species IV is based on the experimental observation that nitrile-type solvents inhibit the reaction (Table 3.1, entry 10). This phenomenon is probably caused by the formation of a catalytic inactive species analogous to IV but with a coordinated acetonitrile. Reaction of IV with a benzyl amine molecule re-generates the catalyst and releases the final nitrile and the second hydrogen equivalent. 


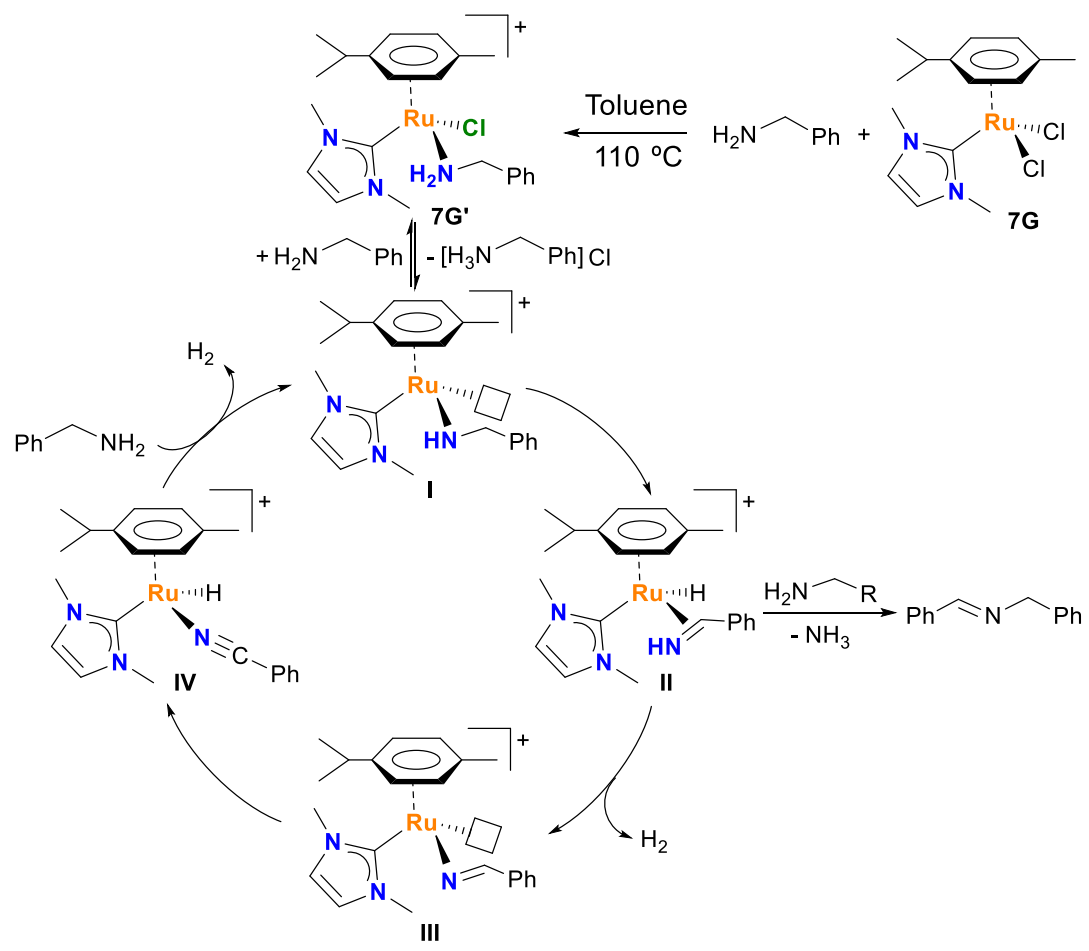

Scheme 3.1 Proposed mechanism for the dehydrogenation of amines

The two hydrogen release steps are the key points of this catalytic cycle. Here, $\mathrm{H}_{2}$ releasing is proposed to happen from ruthenium hydride species with either coordinated imine (II) or nitrile (IV) ligands. The mechanism depicted in Scheme 3.1 is one of the possible scenarios for the acceptorless dehydrogenation of primary amines. Additional experimental and theoretical data is required to understand all the elementary steps of the reaction. 


\subsection{CONCLUSIONS}

Complex $3 \mathrm{C}$ and a series of Ru complexes with different electronic properties 4D, 5E and $6 \mathrm{~F}$ were tested in the dehydrogenation of benzyl amines. The results show that the four complexes are active in this reaction. However, all of them suffer from limited selectivity, without any substantial difference in reactivity among them. Moving from benzyl amines to aliphatic amines has a remarkable effect in the selectivity, as the formation of by-products is avoided using long-chain alkyl amines.

The hybrid material $\mathbf{3 C}$-rGO is active in the dehydrogenation of amines, and can be recycled eight times without any substantial decrease in activity. The hot filtration test shows that $\mathbf{3 C}$ is strongly anchored on the surface of rGO during the catalysis and no activity could be attributed to leached species after filtration at high temperature. After the recycling experiment, a morphological analysis was carried out by TEM. The micrographs reveal that no metal nanoparticles have been formed at the end of the reaction, indicating the molecular nature of the active species.

Finally, a tentative mechanistic has been proposed based on experimental evidences and a relevant catalytic species has been isolated and fully characterised. 


\subsection{REFERENCES}

[1] F. F. Fleming, Nat. Prod. Rep. 1999, 16, 597-606.

[2] L. Cristian, S. Nica, O. D. Pavel, C. Mihailciuc, V. Almasan, S. M. Coman, V. I. Parvulescu, Catal. Sci. Technol. 2013, 3, 2646-2653.

[3] M. A. Nielsen, M. K. Nielsen, T. Pittelkow, Org. Process Res. Dev. 2004, 8, 1059-1064.

[4] H. H. Hodgson, Chem. Rev. 1947, 40, 251-277.

[5] S. I. Murahashi, Y. Imada, in Transition Metals for Organic Synthesis, 2008, pp. 497-507.

[6] R. Reguillo, M. Grellier, N. Vautravers, L. Vendier, S. Sabo-Etienne, J. Am. Chem. Soc. 2010, 132, 7854-7855.

[7] S. Enthaler, D. Addis, K. Junge, G. Erre, M. Beller, Chem. - A Eur. J. 2008, 14, 9491-9494.

[8] T. Li, I. Bergner, F. N. Haque, M. Z. Iuliis, D. Song, R. H. Morris, R. V August, Organometallics 2007, 26, 5940-5949.

[9] D. B. Bagal, B. M. Bhanage, Adv. Synth. Catal. 2015, 357, 883-900.

[10] M. Grellier, S. Sabo-Etienne, Dalton Trans. 2014, 43, 6283-6286.

[11] Z. Wang, J. Belli, C. M. Jensen, Faraday Discuss. 2011, 151, 297-305.

[12] K. N. T. Tseng, A. M. Rizzi, N. K. Szymczak, J. Am. Chem. Soc. 2013, 135, 16352-16355.

[13] L. V. A. Hale, T. Malakar, K. N. T. Tseng, P. M. Zimmerman, A. Paul, N. K. Szymczak, ACS Catal. 2016, 6, 4799-4813.

[14] I. Dutta, S. Yadav, A. Sarbajna, S. De, M. Hölscher, W. Leitner, J. K. Bera, J. Am. Chem. Soc. 2018, 140, 8662-8666.

[15] C. Hammond, M. T. Schümperli, I. Hermans, Chem. - A Eur. J. 2013, 19, 13193-13198.

[16] D. Ainembabazi, N. An, J. C. Manayil, K. Wilson, A. F. Lee, A. M. VoutchkovaKostal, ACS Catal. 2019, 9, 1055-1065.

[17] E. C. Corker, U. V. Mentzel, J. Mielby, A. Riisager, R. Fehrmann, Green Chem. 2013, 15, 928-933. 
[18] K. Yamaguchi, N. Mizuno, Angew. Chemie - Int. Ed. 2003, 42, 1480-1483.

[19] D. Damodara, R. Arundhathi, P. R. Likhar, Adv. Synth. Catal. 2014, 356, 189198.

[20] A. Prades, E. Peris, M. Albrecht, Organometallics 2011, 30, 1162-1167.

[21] W. Chen, J. Egly, A. I. Poblador-Bahamonde, A. Maisse-Francois, S. BelleminLaponnaz, T. Achard, Dalton Trans. 2020, 49, 3243-3252.

[22] A. Primo, M. Puche, O. D. Pavel, B. Cojocaru, A. Tirsoaga, V. Parvulescu, H. García, Chem. Commun. 2016, 52, 1839-1842.

[23] L. Martínez-sarti, S. Díez-gonzález, ChemCatChem 2013, 5, 1722-1724.

[24] P. Csabai, F. Joo, Organometallics 2004, 23, 5640-5643.

[25] S. McGrandle, G. C. Saunders, J. Fluor. Chem. 2005, 126, 449-453.

[26] S. Kumar, A. Narayanan, M. N. Rao, M. M. Shaikh, P. Ghosh, J. Chem. Sci. 2011, 123, 791-798.

[27] T. Achard, J. Egly, M. Sigrist, A. Maisse-François, S. Bellemin-Laponnaz, Chem. - A Eur. J. 2019, 25, 13271-13274.

[28] C. Vicent, D. G. Gusev, ACS Catal. 2016, 6, 3301-3309. 


\section{CHAPTER 4}

Hydrogen Production on-Demand from the Coupling of Alcohols and Silanes Catalysed by Ruthenium and Iridium Complexes Grafted onto Reduced Graphene Oxide

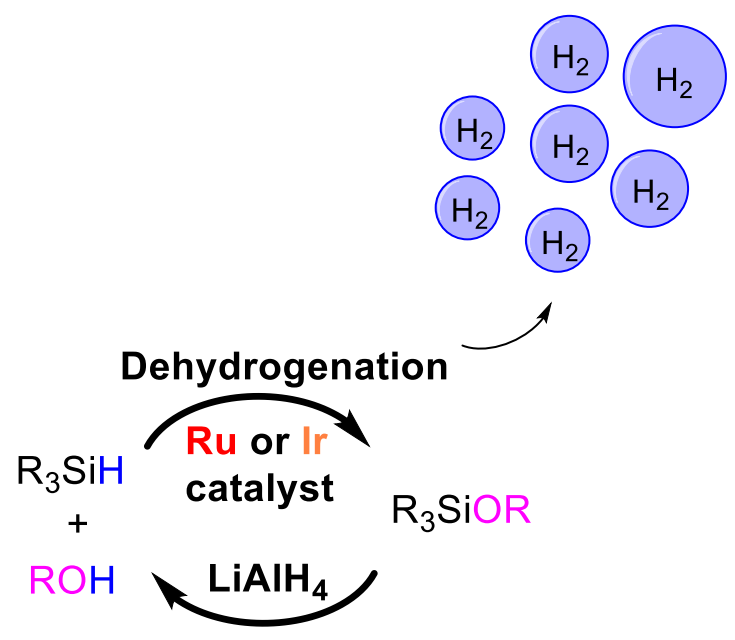

Hydrogenation 



\subsection{INTRODUCTION}

\subsubsection{Liquid organic hydrogen carriers}

Currently, world energy production is based on fossil fuels. However, this situation is no sustainable for a longer time due to factors such as climate change, natural sources depletion and also geopolitical reasons. As an alternative, renewable energies such as solar or wind energy have arisen as powerful tools to mitigate $\mathrm{CO}_{2}$ emissions and provide an alternative energy source. ${ }^{[1,2]}$ The problem with wind and solar light is their fluctuation and intermittent character, which make them unfeasible to fulfil consumption energy requirements. That is why energy storage is a transversal hot topic that has attracted the attention of the scientific community.

Molecular hydrogen is considered as a powerful and clean energy carrier. Hydrogen gas has a high gravimetric energy density $(33.3 \mathrm{KW} \cdot \mathrm{h} / \mathrm{Kg})$. When it is burned or used in a fuel cell the only by-product is water, thus completely avoiding the carbon footprint ligated to present energy generation. ${ }^{[3-5]}$ However, the use of hydrogen as energy vector has the following drawbacks:

- $\mathrm{H}_{2}$ is a gas with an extreme low density $\left(0.0899 \mathrm{Kg} / \mathrm{m}^{3}\right)$ under ambient conditions, which makes necessary the use of high pressure tanks (usually 700 bar) or cryogenic temperatures $\left(-253^{\circ} \mathrm{C}\right)$ to store it in a reasonable amount. $^{[6]}$

- $\mathrm{H}_{2}(\mathrm{~g})$ is extremely flammable and explosive, which makes it difficult to manipulate.

- Elemental hydrogen is not present in the earth crust to any significant extent, so it has to be produced from other forms of energy.

Today $85 \%$ of hydrogen is produced via steam methane reforming (SMR), which liberates enormous amounts of $\mathrm{CO}_{2} \cdot{ }^{[7]}$ For instance, the generation of 1 ton of hydrogen by SMR generates more than 10 tons of $\mathrm{CO}_{2} \cdot{ }^{[8]}$ In an ideal scenario, hydrogen will be obtained from water electrolysis (WE). Nonetheless, today only $4 \%$ of global hydrogen production is obtained from water. ${ }^{[9]}$ The reason is that $\mathrm{H}_{2}$ production from WE is twice more expensive that hydrogen production from SMR. In addition, an essential requirement for the success of WE as the corner stone for energy production is an energy input for the electrolysis coming from solar or wind energies which, again, are characterised by its intermittent character. All these features call for an efficient way to safely store and transport hydrogen. 
In this regard, the storage of hydrogen in the form of liquid organic hydrogen carriers (LOHC) has gained a key position in the development of hydrogen-based technologies (Figure 4.1). ${ }^{[5,10-12]} \mathrm{A}$ LOHC is a system formed by a hydrogen rich molecule ( $\mathrm{Hr}$-LOHC) and a hydrogen-poor counterpart (Hp-LOHC). Hydrogen is loaded in the system by a catalytic hydrogenation of the Hp-LOCH into the Hr-LOHC, using hydrogen coming from the electrolysis of water powered by a renewable energy. Then, owing to its liquid and non-dangerous nature, the Hr-LOCH can be safely stored for a long time or transported to remote areas using the established transport systems for current fuels (e.g. ships, pipelines, trucks). Once in the place of consumption, hydrogen is discharged or released through a catalytic dehydrogenation reaction. This hydrogen is then directly used in a combustion engine or in a fuel cell, generating energy and giving water as the only by-product. In the last stage, the Hp-LOHC is transported back to be hydrogenated again. The process is reversible and multiple hydrogenation/dehydrogenation cycles can be performed without consumption of LOHC.

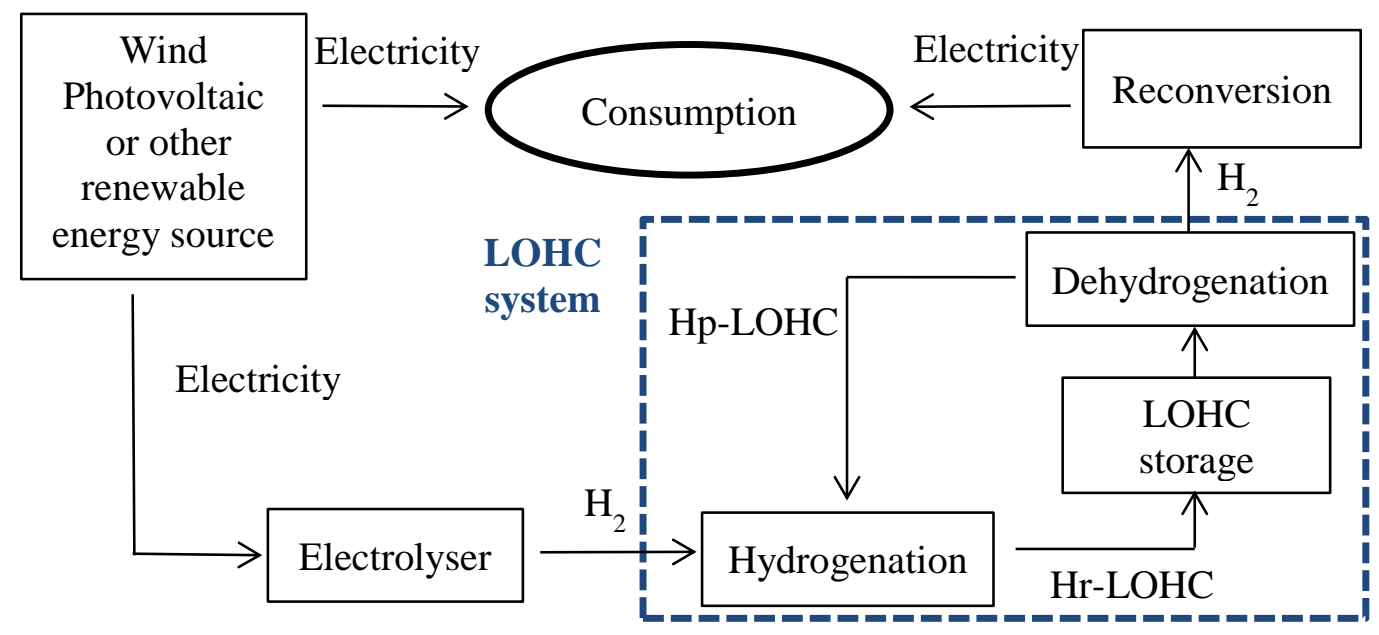

Figure 4.1 Schematic representation of a LOHC. Reproduced with permission from ref [13]

Two important features to take into account when discussing LOHCs are the dehydrogenation temperature (i.e. the temperature at which hydrogen is released from the $\mathrm{Hr}$-LOHC) and the hydrogen storage capacity (HSC), which determines the effective amount of hydrogen that can be carried with the LOHC. For practical purposes, the dehydrogenation temperature should be as low as possible to avoid energy costly processes. In this same regard, the HSC has to be as high as possible to maximise the volume of $\mathrm{H}_{2}$ released in each cycle. Up to now, different organic pairs have been considered as efficient LOHC. The most relevant are included in Table 4.1. 
Table 4.1 Proposed systems as LOHC

Dehydr. ${ }^{\text {eff }} \mathrm{HSC}$

Temp $\left({ }^{\circ} \mathrm{C}\right)(\mathrm{wt} \%)$

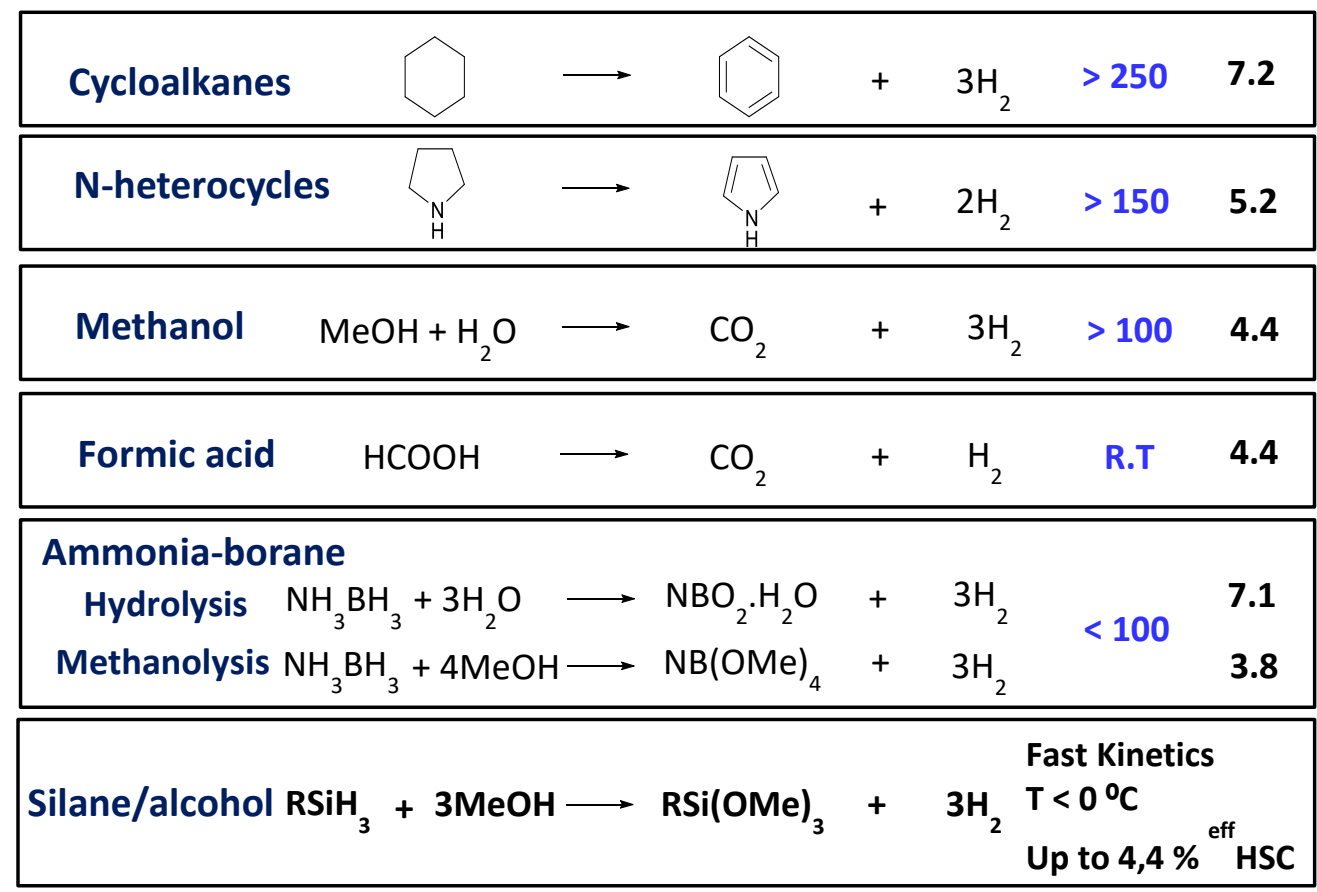

\section{Cycloalkanes}

Studies on LOHC began in early eighties when Taube and co-workers proposed the pair methylcyclohexane/toluene (MCH/TOL) as a hydrogen storage system for onboard applications. ${ }^{[14]}$ After this historical report, the following studies focused in the pair $\mathrm{MCH} / \mathrm{TOL}$, which has a remarkable hydrogen storage capacity (HSC) of 6.1 wt \%. The main drawback of this pair is the high energy input necessary for the highly endothermic dehydrogenation step. To achieve high hydrogen production the reactors usually operate at temperatures around $300-350{ }^{\circ} \mathrm{C}$ to achieve full conversion of $\mathrm{MCH}^{[15]}$ Under this reaction conditions all the components of the system are gaseous. This means that extra purification steps are needed to isolate hydrogen from the gaseous mixture. Additionally, such high temperatures limit its implementation for on-board applications (e.g. cars or trucks) which is one of the large energy consumer sectors. Despite all these limitations, the Japanese corporation Chiyoda have licensed and successfully implemented a pilot plant based on the $\mathrm{MCH} / \mathrm{TOL}$ pair that is capable to generate $50 \mathrm{Nm}^{3} / \mathrm{h}^{[16]}$ 
In order to overcome the problems of the MCH/TOL pair, a LOHC based on decalin/naphtalene was proposed. The system has a higher HSC of $7.2 \mathrm{wt} \%$, but presents two main drawbacks:

- Naphthalene is a carcinogenic substance.

- Naphthalene is a solid under ambient conditions.

- The dehydrogenation of decalin to naphthalene has been reported to be irreversible. ${ }^{[17]}$

Although some reports have established good conversions and selectivities for the dehydrogenation of decalin, ${ }^{[18-20]}$ these two issues prevent the decalin/naphthalene pair to be considered as an efficient hydrogen storage system.

Recent reports in this field focus on the perhydro-dibenzyltoluene/dibenzyltoluene pair (pHDBT/DBT). These two compounds are non-toxic and liquid in a wide range of temperatures (-30 to $390^{\circ} \mathrm{C}$ ). His HSC is $6.2 \mathrm{wt} \%$ and pHDBT shows a lower heat of hydrogenation than $\mathrm{MCH}(65.3 \mathrm{vs} 68.3 \mathrm{~kJ} / \mathrm{mol})$, so the dehydrogenation reaction can be performed at lower temperatures (ca. 250-300 ${ }^{\circ} \mathrm{C}$ ) and ambient pressure. ${ }^{[11]}$ Besides, DBT is still present in the market as heat transfer oils, which make the pHDBT/DBT a good candidate for hydrogen generation in stationary power storage units. In fact, Hydrogenious technologies has pioneered in the implementation of LOHC in Europe, with already commercially available hydrogenation and dehydrogenation units based on this pair. ${ }^{[21]}$

\section{N-heterocycles}

Pursuing the reduction of the high temperatures needed for the dehydrogenation of cycloalkanes, other systems were studied. Pioneering works by Pez at the company Air Products, ${ }^{[22]}$ and Crabtree ${ }^{[23,24]}$ established that the introduction of a heteroatom (and more specifically nitrogen) in the cyclic structure helps to reduce the energy required during the hydrogen release step without a substantial decrease of the HSC.

Among the $\mathrm{N}$-heterocycles studied in this field, perhydro- $\mathrm{N}$-ethylcarbazole and $\mathrm{N}$-ethylcarbazole (pHNEC/NEC) constitute the most studied LOHC pair based on $\mathrm{N}$-heterocycles. The lower dehydrogenation enthalpy of pHNEC $(50 \mathrm{KJ} / \mathrm{mol})$, allows to carry out the dehydrogenation at temperatures of $200-230{ }^{\circ} \mathrm{C}^{[10]}$ As for the decalin/naphthalene pair, one of the drawbacks of pHNEC/NEC is its high melting point. To solve this, eutectic mixtures of different $\mathrm{N}$-alkyl carbazoles have shown a 
melting point of $24{ }^{\circ} \mathrm{C}$ without a dramatic decrease of the HSC. ${ }^{[25]}$ Another issue that still needs to be fully solved is the lability of the $\mathrm{N}$-alkyl bond at the operated temperatures, which leads to some decomposition of the substrates. ${ }^{[10]}$

More recently, Sabo-Etienne and co-workers have proposed the amine/nitrile pair as a potential LOHC. ${ }^{[26]}$ Each fragment of $\mathrm{CH}_{2}-\mathrm{NH}_{2}$ contains a 13.2 wt \% of hydrogen that could be potentially released if it is selectively dehydrogenated to a $\mathrm{C} \equiv \mathrm{N}$ fragment. The easiest amine/nitrile pair would be ethylamine/acetonitrile, which has a remarkable HSC of $8.8 \mathrm{wt} \%$. However, ethylamine has a low boiling point $\left(16.6{ }^{\circ} \mathrm{C}\right)$ which prevents its practical application. Thus, amines with longer chains have to be considered. In this regard, 1,5 diaminopentane seems a good candidate to develop an efficient LOHC based on the amine/nitrile pair.

Albeit with limited examples of systems operating under soft conditions, nitrile hydrogenation is a well-understood process that has applications in current industrial facilities. ${ }^{[27-29]}$ On the contrary, the selective dehydrogenation of amines to produce nitriles can be considered in its infancy, with just three examples of catalysts that are able to efficiently perform this transformation. ${ }^{[30-32]}$ So, while it seems to be a promising area, further research is still needed to develop an efficient LOHC based on the nitrile/amine pair.

\section{Methanol}

Methanol is a readily available chemical that, ideally, can store up to $12.6 \mathrm{wt} \%$ of hydrogen. It is a basic chemical with a worldwide production of more than 95 million metric tons per year. ${ }^{[33]}$ That is why hydrogen storage in the form of methanol would bring a cheap hydrogen carrier with good acceptance in the market and the society.

However, the main problem when using methanol to produce hydrogen is the concomitant generation of 1 mol of $\mathrm{CO}_{2}$ per each mol of $\mathrm{MeOH}$ (eq 1), thus contributing to global warming. Hence, the development of a methanol based economy is directly linked to an efficient capture of $\mathrm{CO}_{2}$ from the atmosphere. As the methanol generation from $\mathrm{CO}_{2}$ and $\mathrm{H}_{2}$ is a well-established process for stationary plants, efficient $\mathrm{CO}_{2}$ recovery from the atmosphere would allow the development of a carbon-neutral fuel based on methanol. ${ }^{[34]}$

$$
\begin{aligned}
\mathrm{MeOH}+\mathrm{H}_{2} \mathrm{O} & \rightleftharpoons 3 \mathrm{H}_{2}+\mathrm{CO}_{2} \quad \text { (eq 1) } \\
\Delta \mathrm{G}^{\circ} & =0.6 \mathrm{KJ} / \mathrm{mol}
\end{aligned}
$$


The reaction shown in eq 1 is highly endothermic $\left(\Delta H^{\circ}=53.3 \mathrm{KJ} / \mathrm{mol}\right)$, therefore a considerable amount of extra energy has to be injected in the system to run the reaction. There are currently some heterogeneous systems that can dehydrogenate a mixture of $\mathrm{MeOH} / \mathrm{H}_{2} \mathrm{O}$ at temperatures of $200-300{ }^{\circ} \mathrm{C}$ and pressures of 20 bar. At these temperatures the reaction takes place in the gas phase, so more efforts are needed to find low-temperature dehydrogenation catalysts, especially for its application in vehicles. In this regard, Beller and co-workers reported a homogeneous $\mathrm{Ru}$ catalyst that is able to dehydrogenate aqueous methanol at a low temperature of $95^{\circ} \mathrm{C}$ and atmospheric pressure. ${ }^{[35]}$ The main drawback of Beller's system is the necessity of a highly basic media $(8 \mathrm{M} \mathrm{KOH})$. Under these conditions, the base captures the $\mathrm{CO}_{2}$ in the form of carbonate which can be an advantage, as greenhouse gas emissions are avoided but, on the contrary, the base is consumed and the aqueous basic waste has to be treated.

\section{Formic Acid}

Formic acid (FA) is a monoprotic carboxylic acid that can store up to 4.4 wt \% of hydrogen. Industrially it is a relevant product with a global capacity of 720000 tons in 2013. ${ }^{[6]}$

The main difference between FA and the other LOHC discussed so far here is that for FA the hydrogen release step is thermodynamically favoured ( $\Delta \mathrm{G}^{0}=-32.9 \mathrm{KJ} / \mathrm{mol}$ ), allowing a faster $\mathrm{H}_{2}$ generation, a key feature for on-board applications. The idea of generating hydrogen from FA dates back to 1967, when Coffrey tested several transition metal complexes in refluxing acetic acid as solvent. ${ }^{[36]}$ However, it was not until 2008 when Beller and Laurenczy independently reported the potential application of FA as a hydrogen storage system. ${ }^{[37,38]}$ After that, hundreds of papers have been published in the field. ${ }^{[6]}$ In general, these systems require the presence of a base or a formate salt as initiator, although base-free systems have also been reported. ${ }^{[39]}$

As in the case of methanol, the main drawback when using FA is the co-generation of $1 \mathrm{~mol}$ of $\mathrm{CO}_{2}$ per mol of FA. A $1 / 1$ mixture of $\mathrm{CO}_{2} / \mathrm{H}_{2}$ is not suitable for direct use in fuel cells, so carbon dioxide needs to be separated from hydrogen using membranes, adding an extra step to the process. ${ }^{[34]}$ Nevertheless, this seems to be a promising technology and the first prototype of bus powered by FA is already under preparation. $^{[40]}$ 
Currently FA is industrially obtained from methanol carbonylation, which implies the use of highly toxic carbon monoxide. To avoid this, alternative routes have been explored for the catalytic production of $\mathrm{FA}$ from $\mathrm{CO}_{2}$ and $\mathrm{H}_{2}$, which will close the cycle for a carbon-neutral process. The main issue is that most of these systems show the best performance in the presence of base. ${ }^{[6]}$ Under basic conditions, pure FA is not directly generated and it has to be separated from the media. Alternatively, base-free systems has also been reported, but still with limited activities. ${ }^{[41-45]}$ Another drawback to completely consider the $\mathrm{FA} / \mathrm{CO}_{2}$ pair as a $\mathrm{LOHC}$ is the need of efficient systems to capture $\mathrm{CO}_{2}$ from the atmosphere; otherwise this pair will be unsuitable to avoid global warming.

\section{Ammonia-Borane}

Ammonia-Borane has been proposed as an efficient energy carrier system. In an ideal situation, it has a high HSC of up to 7.1 wt \%. Ammonia-borane dehydrogenation is a strongly favoured process $\left(\Delta \mathrm{G}^{\circ}=-56 \mathrm{KJ} / \mathrm{mol}\right){ }^{[46]}$ and there is an important number of catalysts that can perform the dehydrogenation at fast rates and temperatures below $100{ }^{\circ} \mathrm{C} .{ }^{[47-49]}$ One problem that is bound to the use of ammonia-borane for $\mathrm{H}_{2}$ generation is the solid nature of $\mathrm{NH}_{3}-\mathrm{BH}_{3}$ under ambient conditions. That is why a solvent is usually required to run these reactions, which affects the overall HSC of the system. ${ }^{[46]}$

The main issue when using ammonia-borane as a LOHC is the regeneration process. The dehydrogenation of ammonia-borane gives polymers or stable borates that are difficult to hydrogenate back to ammonia borane. ${ }^{[50]}$ This hydrogenation is a highly endothermic reaction that can be in principle driven at high temperatures. However, the product decomposes at temperatures even below the melting point. ${ }^{[51]}$ That is why more research is needed in this area to consider ammonia-borane as a LOHC.

\subsubsection{The silane-alcohol pair}

Being all these systems shortly reviewed, chapter 4 of the present thesis deals with the study of the dehydrogenative coupling of hydrosilanes and alcohols as a potential LOHC system (Scheme 4.1). 


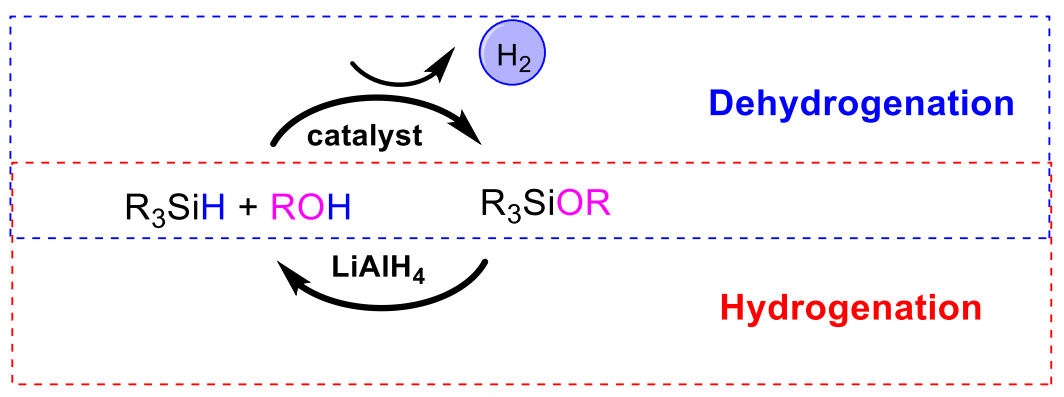

Scheme 4.1 Silane-Alcohol based LOHC

The coupling between hydrosilanes and alcohols has been extensively studied. ${ }^{[52]}$ The reaction generates hydrogen and a silyl ether that find extensive applications in materials science as polymer precursors or for the generation of silica-based mesoporous materials. ${ }^{[53]}$ Silyl ethers are also used for material surface modification or as silylating reagents ${ }^{[54]}$

Traditionally, silyl ethers are obtained from chlorosilanes and alcohols in basic media (Figure $4.2, A) \cdot{ }^{[5]}$ As a consequence a chlorinated waste is generated, which is not convenient in terms of atom efficiency. As an alternative, direct alcoholisis of hydrosilanes is a more suitable approach for the synthesis of silyl ethers (Figure 4.2, B). In addition, hydrogen gas is released as the only by-product. However, alcohols are not nucleophilic enough to promote this transformation, and a catalyst is needed.

A
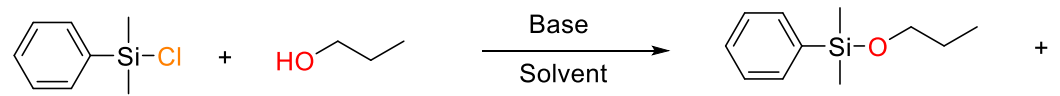

$\mathrm{H}-\mathrm{Cl}$

B
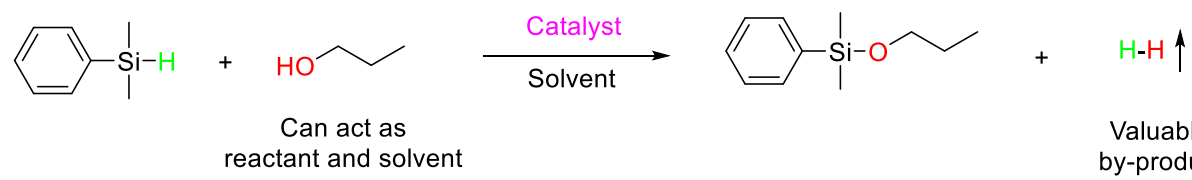

Valuable by-product

Figure 4.2 Traditional (A) vs Catalytic (B) synthesis of silyl ethers

Despite all the research done towards the development of new catalysts for the dehydrogenative coupling between silanes and alcohols, the potential applications from a hydrogen generation viewpoint remain almost unexplored. ${ }^{[56,57]}$ The silane/alcohol coupling can be considered as a potential LOHC for on-board applications if some conditions are fulfilled: 
- The release of hydrogen has to be quantitative and rapid at low to moderate temperatures.

- The HSC of the system has to be large enough to allow its implementation in mobile systems.

- The dehydrogenation step has to be mediated by a catalyst to avoid spontaneous gas evolution.

- The catalyst should better be heterogeneous, stable and highly recyclable, especially if it is based in an expensive and scarce noble metal.

- The final silyl ether should be reconverted to the former hydrosilane, thus closing the cycle.

There is a plethora of catalysts of different nature that are active in the dehydrogenative coupling between hydrosilanes and alcohols. Simple and commercially available bases such as $\mathrm{NaOH}^{[58]} \mathrm{Cs}_{2} \mathrm{CO}_{3}{ }^{[59]}$ or KOtBu${ }^{[60]}$ can promote the reaction under catalytic conditions. $\mathrm{B}\left(\mathrm{C}_{6} \mathrm{~F}_{5}\right)_{3}$ and other Lewis acids can also catalyse the dehydrogenative coupling between hydrosilanes and alcohols. ${ }^{[61,62]}$ In general basic/acid catalysis have the advantage of being cheap and commercially available, but on the contrary, high loadings are usually needed, with relatively long reaction times and separation of the catalyst add an extra step to the procedure.

Metal complexes have been widely used to carry out the coupling between hydrosilanes and alcohols. First row metal compounds based on $\mathrm{Mn},{ }^{[63,64]} \mathrm{Fe},{ }^{[65-67]}$ $\mathrm{Cu}^{[68]} \mathrm{Ni}^{[69]}$ and $\mathrm{Zn}^{[70,71]}$ can efficiently catalyse this transformation. The major drawback when using these species is that high temperatures, high catalyst loadings or long reaction times are needed. Alternatively, second and third row compounds of $\mathrm{Pd}^{[72]} \mathrm{Ru}^{[73,74]} \mathrm{Ir}^{[75,76]}, \mathrm{Rh}^{[77]}$ or $\mathrm{Au}^{[78]}$ efficiently catalyse the silane-alcohol coupling at room temperature, without additives and at fast rates.

\subsubsection{Reduction of silyl-ethers}

The basis for the success of the silane/alcohol pair as a LOHC relies on the regeneration of the hydrosilane. Currently, hydrosilanes are obtained by reduction of the corresponding silyl ethers with metal hydrides such as $\mathrm{LiAlH}_{4}$. Although this method is currently used in industry and operates under relatively mild conditions, ${ }^{[79]}$ a "greener" method is highly desirable.

In 2019 the group of Shimada reported the reduction of methoxy(dimethyl)phenyl silane using 4,4,5,5-tetramethyl-1,3,2-dioxaborolane (HBPin) as a milder hydride source and a yttrium catalyst (Figure $4.3, A) .{ }^{[80]}$ With this platform a $57 \%$ yield was 
obtained at $100{ }^{\circ} \mathrm{C}$ after 24 hours. Despite the moderate yield, this system constitutes the first example for a reduction of a silyl ether in the absence of lithium aluminium hydride. Few months later, the same group developed an organocatalytic system that is able to reduce a wide variety of silyl ethers containing one to three Si-O bonds, at room temperature and using $\mathrm{BH}_{3} \cdot \mathrm{THF}$ as the final reductant (Figure $4.3, \mathrm{~B}){ }^{[81]}$

A)

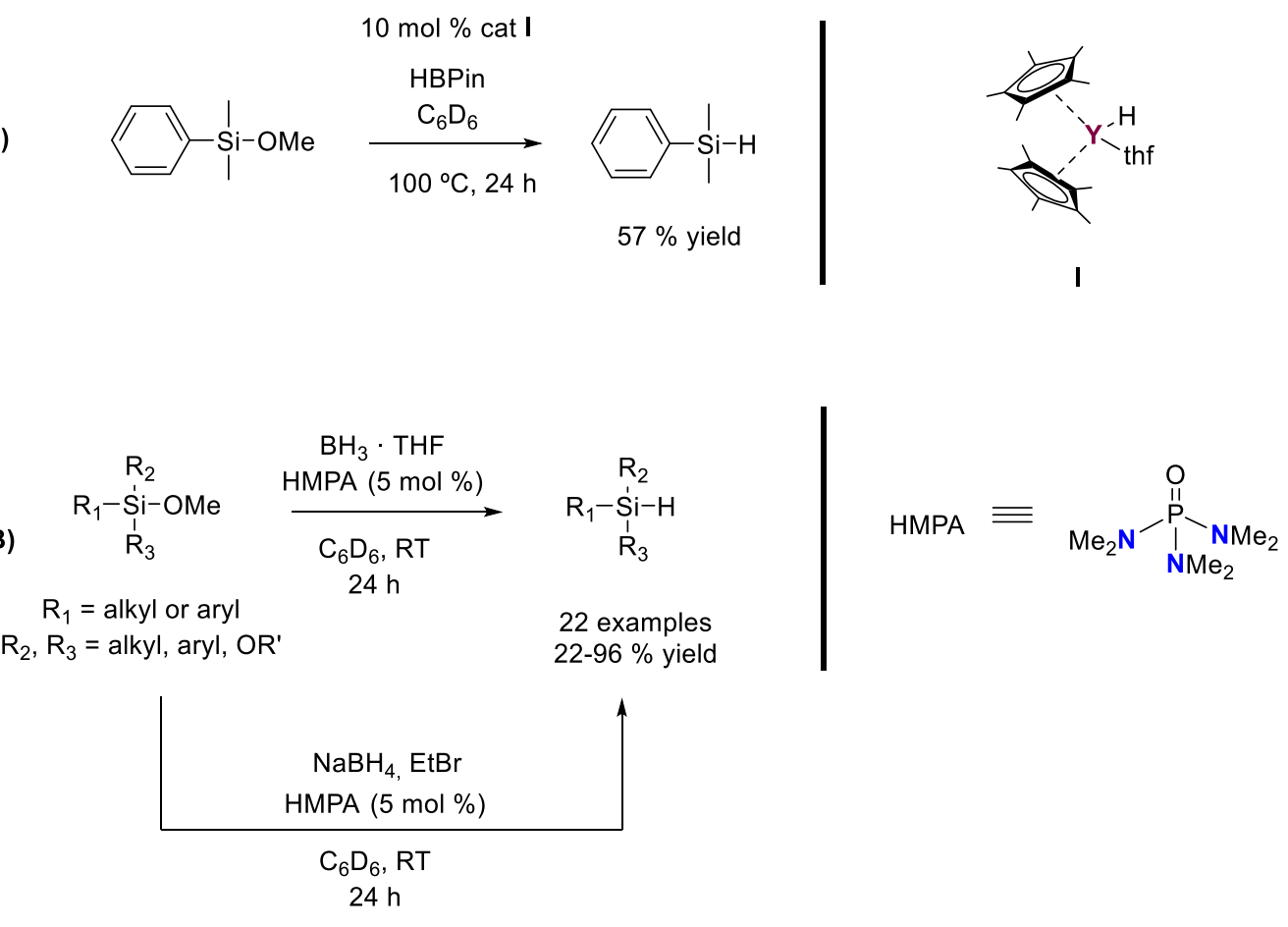

Figure 4.3 Catalytic systems for the reduction of silyl-ethers

Alternatively, hydrosilanes can be obtained from the reduction of halosilanes, which can be easily obtained from silyl-ethers. ${ }^{[82]}$ The lower bond dissociation energy of the $\mathrm{Si}-\mathrm{Cl}$ bond $(456 \mathrm{KJ} / \mathrm{mol})$ in front of $\mathrm{Si}-\mathrm{O}$ bond $(798 \mathrm{KJ} / \mathrm{mol})$ renders a less disfavoured thermodynamic process. The group of Shimada has pioneered in the development of this transformation. In 2017 they reported an Ir (I) precatalyst that is able to reduce halosilanes to hydrosilanes in the presence of a base (Figure 4.4). ${ }^{[83]}$ Notably, hydrogen could be used as the terminal reductant under soft conditions ( 4 bar of $\mathrm{H}_{2}$ and RT). However, only less available iodo-silanes can be easily activated, bromo-silanes reacted very slowly and the easily accessible chloro-silanes remained unreacted. 

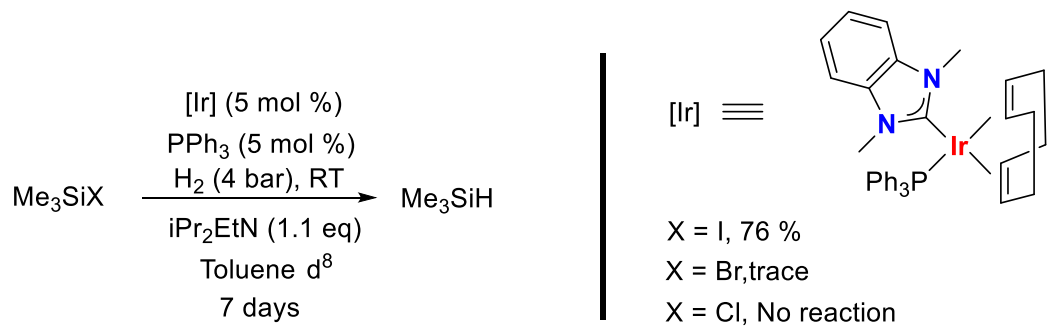

Figure 4.4 Reduction of halosilanes mediated by Ir (I) reported by Shimada and coworkers

In this work they hypothesize that the $\operatorname{Ir}(\mathrm{I})$ precatalyst suffers from an oxidative addition of hydrogen and decoordination of cyclooctadiene, giving raise to the actual catalytically active Ir (III) species. Then, the same group reported a Ir (III) complex with a pyridylidene amide ligand that can hydrogenate halosilanes to produce hydrosilanes. ${ }^{[84]}$ Chlorosilanes could be used as the starting material to generate the iodo-silane by in situ halide scrambling. Using this system different mono- and di-chlorosilanes were hydrogenated in moderate to good yields (Figure 4.5). In addition, selective monohydrogenolysis of di- and trichlorosilanes similarly proceeded, resulting in the formation of chlorohydrosilanes as synthetically useful building blocks.

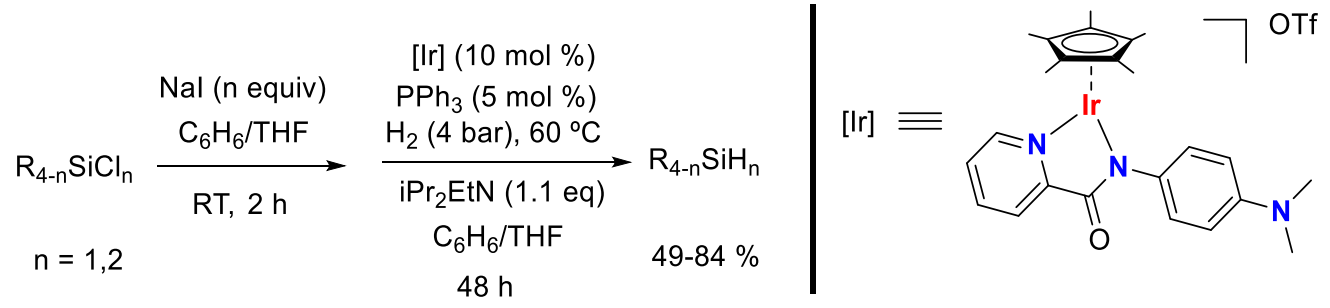

Figure 4.5 Reduction of halosilanes mediated by Ir (III) reported by Shimada and coworkers

More recently Schneider reported the direct activation of chlorosilanes using a bifunctional catalyst based on a cheaper metal such as ruthenium (Figure 4.6). ${ }^{[85]}$ This platform allows the generation of mono- and di-hydrosilanes at low catalyst loadings and under soft reaction conditions ( 4 bar $\mathrm{H}_{2}, \mathrm{RT}$ ). 


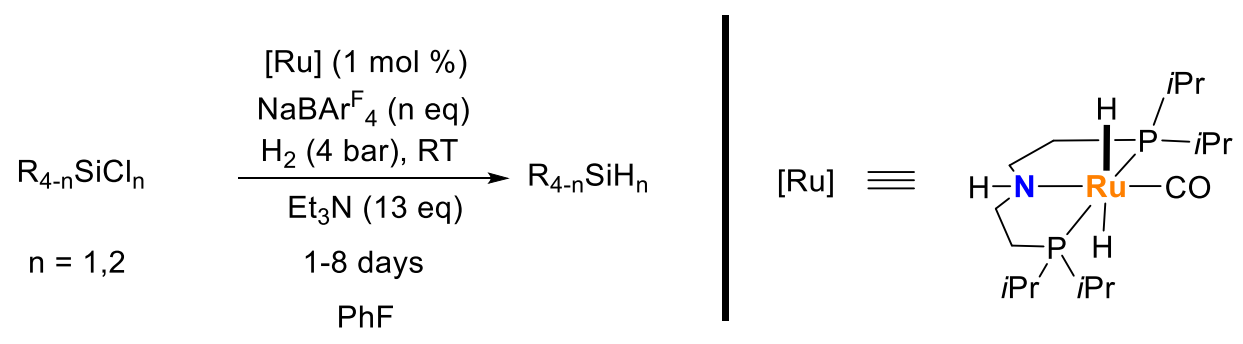

Figure 4.6 Reduction of halosilanes mediated by Ru (II) reported by Schneider and coworkers

The presence of sodium tetrakis [3,5-bis(trifluoromethyl)phenyl] borate] ( $\left.\mathrm{NaBAr}_{4}{ }_{4}\right)$ was essential to make the reaction run. Supported by NMR experiments the authors suggest that $\left[\mathrm{Me}_{3} \mathrm{SiNEt}_{3}\right]^{+}$is the actual substrate initially formed before the hydrogenolysis. This cation is stabilised by the sterically big $\mathrm{BAr}_{4}{ }_{4}$ anion which proved to be key. Salts based on other weakly coordinating anions such as $\mathrm{NaOTf} \mathrm{KPF}_{6}$, $\mathrm{NaBPh}_{4}, \mathrm{NaSbF}_{6}$ or $\mathrm{NaBF}_{4}$ resulted in no conversion even after extended periods.

Taking all of this into account, this chapter deals with the study of the dehydrogenative coupling between alcohols and hydrosilanes mediated by Ru and Ir complexes anchored onto reduced graphene oxide. The stability and recyclability of the Ir and Ru hybrid materials is studied, and a plausible mechanism for the reaction is proposed based on experimental evidences and DFT calculations. 


\subsection{RESULTS AND DISCUSSION}

The dehydrogenative coupling between alcohols and hydrosilanes was tested using ruthenium and iridium complexes (Figure 4.7):

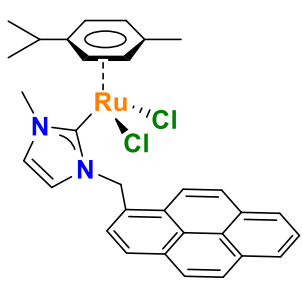

$3 C$

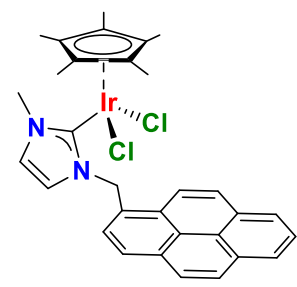

$8 \mathrm{C}$

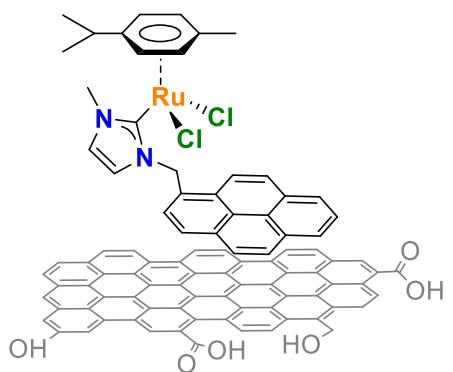

3C-rGO

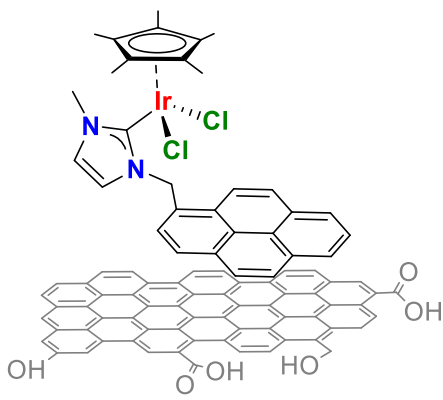

8C-rGO

Figure 4.7 Molecular complexes and hybrid materials used in this chapter

The synthesis of the ruthenium molecular complex $(3 \mathrm{C})$ and the related hybrid material (3C-rGO) is described in chapter 2 and the synthesis and characterisation of the iridium complex $(\mathbf{8 C})$ and its grafting onto graphene (8C-rGO) is discussed in the following section.

\subsubsection{Synthesis and characterisation of an Iridium complex bearing a pyrene tag and immobilisation onto graphene}

In an analogy to the synthesis of $\mathbf{3 C}$, the synthesis of the iridium complex $\mathbf{8 C}$ was attempted by transmetallation. Under these reaction conditions, the ortometallated complex $\mathbf{8 \mathbf { C } ^ { \prime }}$ was obtained as the major product (Figure 4.8). ${ }^{[86]}$ The non-orthometallated compound $\mathbf{8 C}$ was obtained by deprotonation of ligand precursor $\mathbf{C}$ with potassium tert-butoxide and metalation at room temperature. $\mathbf{8 C}$ could be then converted into $\mathbf{8} \mathbf{C}^{\prime}$ by addition of a second equivalent of $t$ BuOK. 

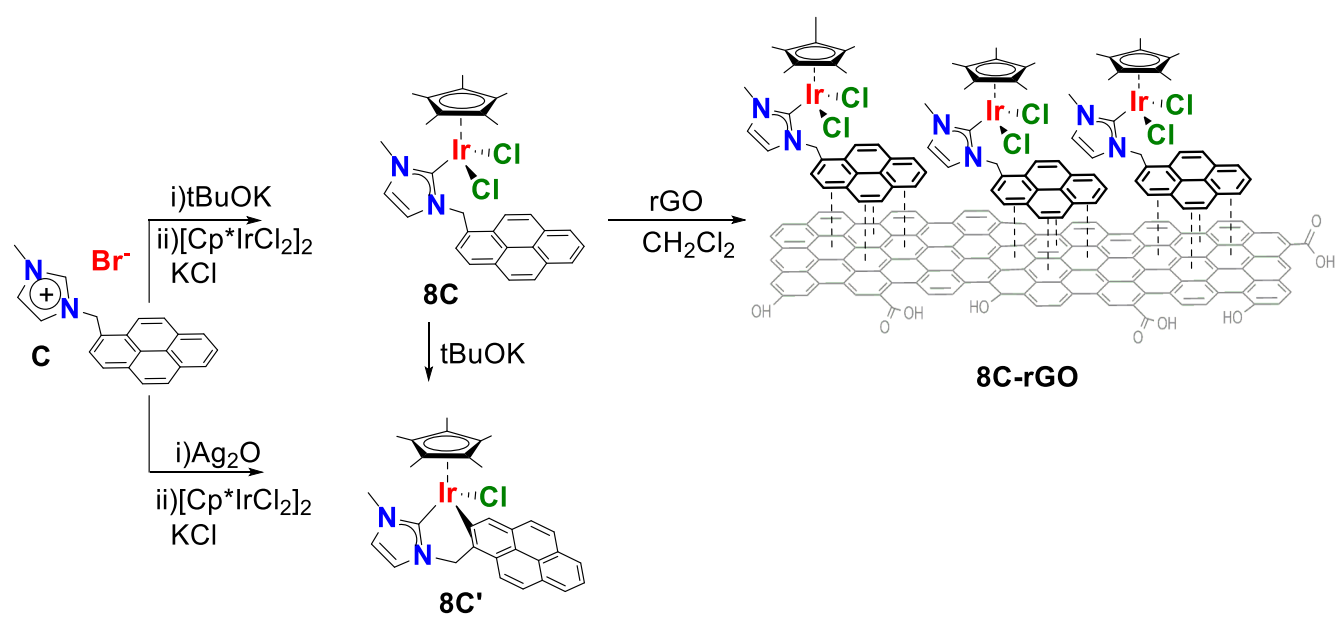

Figure $\mathbf{4 . 8}$ Synthesis of $\mathbf{8 C}, \mathbf{8} \mathbf{C}^{\prime}$ and $\mathbf{8 C}-\mathrm{rGO}$

The two new complexes $\mathbf{8 C}$ and $\mathbf{8 C ^ { \prime }}$ were fully characterised by ${ }^{1} \mathrm{H} N M R,{ }^{13} \mathrm{C} N M R$, ESI-MS, HRMS, elemental analysis and X-Ray Diffraction. The first evidence that the metalation had taken place was the disappearance of the acidic signal due to the $\mathrm{NCHN}$ proton of the imidazolium salt in the ${ }^{1} \mathrm{H}$ NMR spectrum. The formation of complexes $\mathbf{8 C}$ and $\mathbf{8} \mathbf{C}^{\prime}$ was further confirmed by ${ }^{13} \mathrm{C} N \mathrm{NMR}$, where the signals for the carbenic carbons could be observed at 157.1 and 156.3 ppm respectively. HRMS spectra of $\mathbf{8 C}$ and $\mathbf{8 C ^ { \prime }}$ show base peaks for the fragment $[\mathrm{M}-\mathrm{Cl}]^{+}$at 659.1803 and 623.2044 a.m.u respectively, which confirm the proposed molecular composition of the complexes based on the mass/charge relation and the isotopic pattern. All the information regarding the characterisation of these new compounds is included in chapter 6 .

Complex $8 \mathrm{C}$ was grafted onto graphene using the methodology previously described by us. ${ }^{[87]}$ The graphene was first sonicated in dichloromethane for thirty minutes, then the complex was added and the mixture sonicated for ten minutes, and finally stirred overnight at room temperature. Then, the black solid was filtered off and washed with dichloromethane, obtaining the hybrid material $\mathbf{8 C}$-rGO. The new material was fully characterised by TEM, UV-Vis and X-ray Photoelectron Spectroscopy (XPS) (Chapter 6). The exact metal content of iridium loaded onto rGO (6.5 wt \%) was determined by digestion of the samples in nitric acid followed by ICP-MS.

Analysis by XPS provides evidence of the molecular structure of complex $8 \mathrm{C}$ on the surface of graphene. A comparative XPS analysis of complex $8 \mathrm{C}$ and the hybrid 
material 8C-rGO shows the characteristic core-level peaks of N1s and Ir4f at the same binding energy (Figure 4.9). XPS analysis shows that the mild conditions used during the immobilisation process preserved the intrinsic properties of both the iridium complex and graphene.
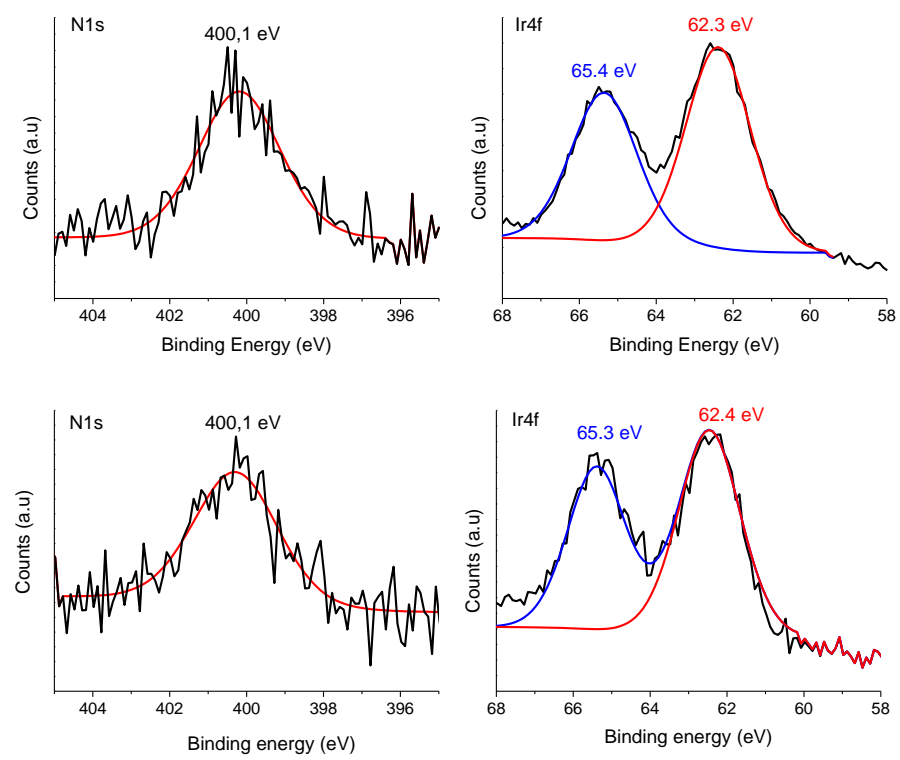

Figure 4.9 Comparative XPS analysis for $8 \mathrm{C}$ (top) and 8C-rGo (bottom)

\subsubsection{Hydrogen production from the coupling between silanes and alcohols catalysed by ruthenium complexes anchored onto rGO}

The catalytic properties of compound $\mathbf{3 C}$ and the related hybrid material $\mathbf{3 C}-\mathrm{rGO}$ were tested in the dehydrogenative coupling between hydrosilanes and alcohols. The optimization of the reaction conditions was performed using dimethylphenyl silane as model substrate (Table 4.2). The $\left[\mathrm{Ru}\left(p\right.\right.$-cym) $\left(\mathrm{NHC}^{-} \mathrm{Cl}_{2}\right]$ compound $3 \mathrm{C}$ is air stable so the reactions can be performed in a round bottom flask under air. The alcohols were used as received without any special precaution. In a standard experiment, $\mathbf{3 C}$ was dissolved in an appropriate alcohol $(1 \mathrm{~mL})$ at $30{ }^{\circ} \mathrm{C}$. The alcohol is used as reactant and solvent for the reaction. Then the hydrosilane was added and the reaction immediately started to evolve hydrogen. The amount of generated hydrogen was quantified by using an inverted burette filled with water. 
Table 4.2 Optimization of reaction conditions in the dehydrogenative coupling of silanes and alcohols catalysed by $3 \mathrm{C}$

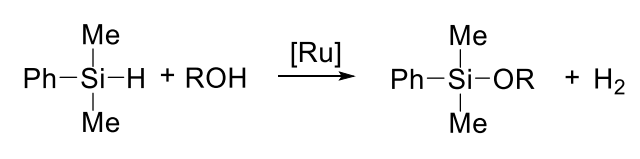

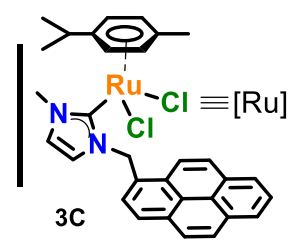

\begin{tabular}{cccccc}
\hline Entry & {$[\mathrm{Ru}](\mathbf{m o l} \%)$} & $\mathbf{T}\left({ }^{\circ} \mathbf{C}\right)$ & $\mathbf{R O H}$ & ${\text { Yield }(\%)^{\mathbf{a}}}^{\mathbf{H}_{\mathbf{2}}(\mathbf{m L})^{\mathbf{b}}}$ \\
\hline 1 & - & 50 & $\mathrm{MeOH}$ & 0 & 0 \\
2 & 1 & 30 & $\mathrm{MeOH}$ & 100 & 22.2 \\
3 & 1 & 30 & $\mathrm{EtOH}$ & $100(93)$ & 22.0 \\
4 & 1 & 30 & $n \mathrm{PrOH}$ & $100(90)$ & 21.7 \\
5 & 1 & 30 & $n \mathrm{BuOH}$ & $100(92)$ & 22.3 \\
6 & 0.5 & 30 & $\mathrm{MeOH}$ & 100 & 22.1 \\
7 & 0.5 & 30 & $\mathrm{EtOH}$ & $100(94)$ & 22.1 \\
8 & 0.5 & 30 & $n \mathrm{PrOH}$ & $100(86)$ & 21.8 \\
9 & 0.5 & 30 & $n \mathrm{BuOH}$ & $100(89)$ & 21.2 \\
10 & 0.1 & 30 & $\mathrm{MeOH}$ & 100 & 22.0 \\
11 & 0.1 & 30 & $\mathrm{EtOH}$ & $94(93)$ & 21.1 \\
12 & 0.1 & 30 & $n \mathrm{PrOH}$ & $45(37)$ & 10.6 \\
13 & 0.1 & 30 & $n \mathrm{BuOH}$ & 10 & 2.7 \\
14 & 0.5 & 0 & $\mathrm{MeOH}$ & 97 & 20.4
\end{tabular}

Reaction conditions: $\mathrm{PhMe}_{2} \mathrm{SiH}(1.0 \mathrm{mmol})$, ruthenium complex $3 \mathrm{C}, 1 \mathrm{~mL}$ of $\mathrm{ROH}$ for $10 \mathrm{~min}$. [a] Yields of silyl ether determined by GC analysis using anisole as the internal standard. Isolated yields in parenthesis determined by ${ }^{1} \mathrm{H}$ NMR spectroscopic analysis using 1,3,5-trimethoxy benzene as external standard. [b] Volume of hydrogen gas collected using an inverted burette.

With a catalyst loading of $1 \mathrm{~mol} \%$ the reaction is complete in short times using $\mathrm{MeOH}, \mathrm{EtOH}, \mathrm{PrOH}$ or $\mathrm{BuOH}$ as solvent. (Entries 2-5, Table 4.2). The silyl ether formed was isolated by simple evaporation of the solvent in excellent isolated yields and selectivity. The hydroxosilane that may be formed from the nucleophilic attack of the water present in the non-dry solvent used was not observed, showing the high selectivity of $\mathbf{3 C}$. $^{[76]}$

Encouraged by these results, the amount of metal complex was reduced up to $0.5 \mathrm{~mol} \%$, giving for all cases quantitative yields in short reaction times (entries 6-9). 
For instance, the reaction between $\mathrm{MeOH}$ and dimethylphenyl silane was complete in less than $30 \mathrm{~s}$ at $30{ }^{\circ} \mathrm{C}$ (vide infra). Metal loading could be further reduced up to $0.1 \mathrm{~mol} \%$, affording quantitative yields for $\mathrm{MeOH}$ and $\mathrm{EtOH}$ (entries 10 and 11). On the contrary, at this loading $n \mathrm{PrOH}$ and $n \mathrm{BuOH}$ gave moderate to low yields, showing the steric effect of lengthen the aliphatic chain of the alcohol.

Catalyst $\mathbf{3 C}$ is very active for the dehydrogenative coupling of alcohols and silanes, and the reaction is very fast even at $0{ }^{\circ} \mathrm{C}$ (entry 14). An study of the reaction at different temperatures show that quantitative yield of $\mathrm{H}_{2}$ is obtained in less than one minute even at $-25{ }^{\circ} \mathrm{C}$ (Figure 4.10). This result indicates the high activity of $\mathbf{3 C}$ in the dehydrogenative coupling between silanes and alcohols.
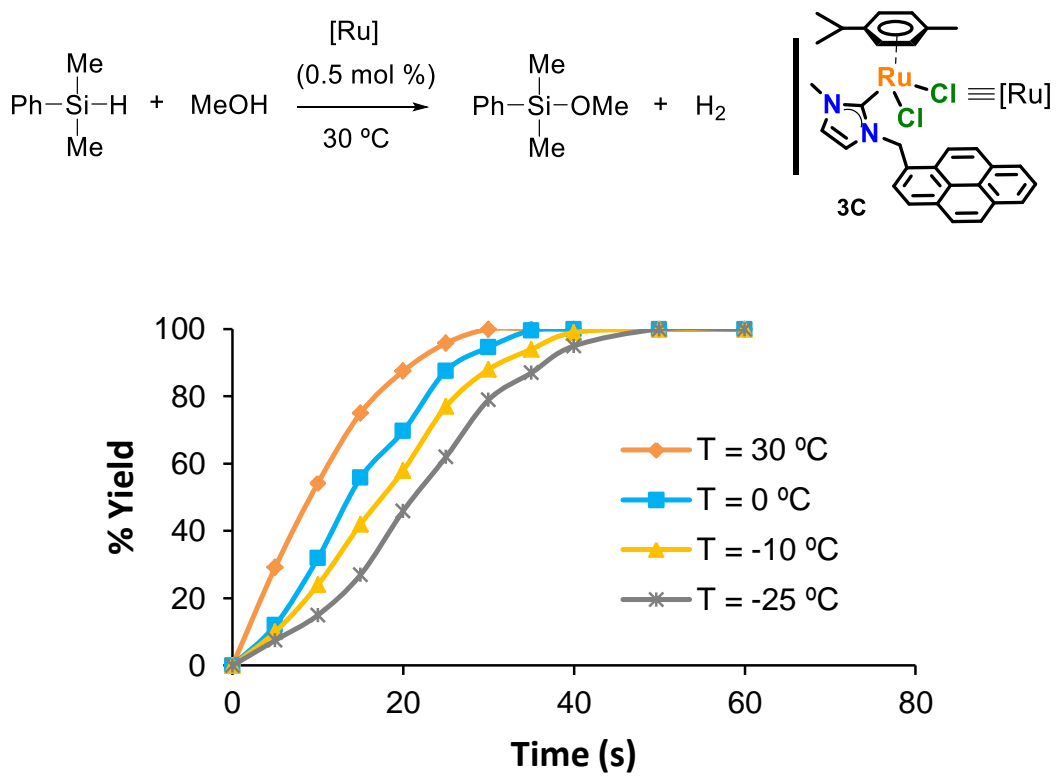

Figure 4.10 Reaction profile at different temperatures. Conditions: $1.0 \mathrm{mmol}$ of dimethylphenyl silane, $1 \mathrm{~mL}$ of $\mathrm{MeOH}$ and $3 \mathrm{mg}$ of $\mathbf{3 C}(0.5 \mathrm{~mol} \%)$. Yield corresponds to the amount of hydrogen collected using an inverted burette

The reaction scope using a variety of hydrosilanes and alcohols was evaluated using complex 3C (Table 4.3). Primary, secondary and tertiary hydrosilanes could be completely dehydrogenated at room temperature $\left(30^{\circ} \mathrm{C}\right)$ producing three, two or one mol of $\mathrm{H}_{2}$ per mol of hydrosilane respectively. The silyl ether products were easily isolated by simple solvent evaporation in excellent purity and yields. 
Table 4.3 Reaction scope for the dehydrogenative coupling of silanes and alcohols catalysed by $3 \mathrm{C}$

\begin{tabular}{|c|c|c|c|c|c|}
\hline \multirow{2}{*}{$\begin{array}{l}\mathrm{R}_{4-n} \mathrm{SiH}_{\mathrm{n}} \\
\text { Entry }\end{array}$} & \multirow{2}{*}{$\begin{array}{l}n+\mathrm{n} \mathrm{R} O H \\
\text { [Ru] (mol \%) }\end{array}$} & $\begin{array}{c}{[\mathrm{Ru}]} \\
\underset{30{ }^{\circ} \mathrm{C}}{(0.5 \mathrm{~mol} \%)}\end{array}$ & $\mathrm{R}_{4-\mathrm{n}} \mathrm{Si}\left(\mathrm{OR}^{\prime}\right)_{\mathrm{n}}+\mathrm{n} \mathrm{H}_{2}$ & \multicolumn{2}{|c|}{$>$ (O) } \\
\hline & & Silane & Alcohol & 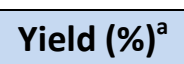 & $\mathrm{H}_{2}(\mathrm{~mL})^{\mathrm{b}}$ \\
\hline 1 & 1 & $\mathrm{Ph}_{2} \mathrm{SiH}_{2}$ & $\mathrm{EtOH}$ & 100 (99) & 44.1 \\
\hline 2 & 1 & $\mathrm{Ph}_{2} \mathrm{SiH}_{2}$ & $n \mathrm{BuOH}$ & 100 (94) & 43.8 \\
\hline 3 & 0.1 & $\mathrm{Ph}_{2} \mathrm{SiH}_{2}$ & $\mathrm{EtOH}$ & 55 & 24.6 \\
\hline 4 & 0.15 & $\mathrm{Ph}_{2} \mathrm{SiH}_{2}$ & $\mathrm{EtOH}$ & 70 & 31.0 \\
\hline 5 & 0.2 & $\mathrm{Ph}_{2} \mathrm{SiH}_{2}$ & $\mathrm{EtOH}$ & 84 & 36.6 \\
\hline 6 & 0.3 & $\mathrm{Ph}_{2} \mathrm{SiH}_{2}$ & $\mathrm{EtOH}$ & 100 & 44.5 \\
\hline 7 & 0.15 & $\mathrm{Ph}_{2} \mathrm{SiH}_{2}$ & $\mathrm{MeOH}$ & 87 & 37.0 \\
\hline 8 & 0.2 & $\mathrm{Ph}_{2} \mathrm{SiH}_{2}$ & $\mathrm{MeOH}$ & 100 & 43.9 \\
\hline 9 & 0.2 & $\mathrm{Ph}_{2} \mathrm{SiH}_{2}$ & $n \mathrm{BuOH}$ & 32 & 15.2 \\
\hline 10 & 0.3 & $\mathrm{Ph}_{2} \mathrm{SiH}_{2}$ & $n \mathrm{BuOH}$ & 54 & 23.2 \\
\hline 11 & 0.5 & $\mathrm{Ph}_{2} \mathrm{SiH}_{2}$ & $n \mathrm{BuOH}$ & $100(95)$ & 44.2 \\
\hline 12 & 0.3 & $\mathrm{Ph}_{2} \mathrm{SiH}_{2}$ & $n \mathrm{PrOH}$ & 90 & 39.4 \\
\hline $13^{c}$ & 0.05 & $\mathrm{PhSiH}_{3}$ & $\mathrm{MeOH}$ & 34 & 10.1 \\
\hline $14^{c}$ & 0.1 & $\mathrm{PhSiH}_{3}$ & $\mathrm{MeOH}$ & 93 & 31.2 \\
\hline $15^{c}$ & 0.5 & $\mathrm{PhSiH}_{3}$ & $\mathrm{MeOH}$ & 100 & 33.5 \\
\hline $16^{c}$ & 0.1 & $\mathrm{PhSiH}_{3}$ & $n \mathrm{PrOH}$ & 92 & 30.9 \\
\hline $17^{c}$ & 0.1 & $\mathrm{PhSiH}_{3}$ & $n \mathrm{BuOH}$ & 84 & 28.2 \\
\hline $18^{d}$ & 1 & $\mathrm{Ph}_{3} \mathrm{SiH}$ & $\mathrm{MeOH}$ & 100 & 22.0 \\
\hline $19^{d}$ & 1 & $\mathrm{Ph}_{3} \mathrm{SiH}$ & $\mathrm{EtOH}$ & 78 & 16.3 \\
\hline $20^{d}$ & 1 & $\mathrm{Ph}_{3} \mathrm{SiH}$ & $n \mathrm{BuOH}$ & 44 & 8.7 \\
\hline 21 & 0.5 & $\mathrm{PhMe}_{2} \mathrm{SiH}$ & $\mathrm{PhCH}_{2} \mathrm{OH}$ & 100 & 22.2 \\
\hline
\end{tabular}

Reaction conditions: Hydrosilane $(1.0 \mathrm{mmol})$, ruthenium complex $3 \mathrm{C}, 1 \mathrm{~mL}$ of ROH for $10 \mathrm{~min}$ at $30{ }^{\circ} \mathrm{C}$. [a] Yields of silyl ether determined by GC analysis using anisole as the internal standard. Isolated yields in parenthesis determined by ${ }^{1} \mathrm{H}$ NMR spectroscopic analysis using 1,3,5-trimethoxybenzene as external standard. [b] Volume of hydrogen gas collected using an inverted burette. [c] $\mathrm{PhSiH}_{3}(0.5 \mathrm{mmol})$. [d] At $45^{\circ} \mathrm{C}$ for 10 hours. 
Primary and secondary hydrosilanes could be efficiently dehydrogenated using $\mathrm{MeOH}$ as the alcohol at low catalyst loadings (0.1 - $0.2 \mathrm{~mol} \%$, entries 8 and 14). When the aliphatic chain of the alcohol was increased, the catalyst loading required was slightly increased $(0.3-0.5 \mathrm{~mol} \%$, view entries $3-6$ and $9-11)$ in order to obtain complete conversions. In the case of bulky tertiary hydrosilanes (such as triphenyl silane), the temperature $\left(45^{\circ} \mathrm{C}\right)$ and the reaction time $(10 \mathrm{~h})$ had to be increased to accomplish complete conversion using $\mathrm{MeOH}$ as the alcohol (entry 18). With this substrate, an important steric effect related to the length of the aliphatic chain in the alcohol was observed. When using ethanol a $78 \%$ yield was obtained after 10 hours (entry 19) and a moderate $44 \%$ yield was accomplished when using butanol (entry 20).

Recyclability studies with 3C-rGO and catalyst stability

An important issue to consider the silane-alcohol pair as a promising LOHC is the long-term stability and recyclability of the catalyst. The stability of $\mathbf{3 C}$ was tested against successive injections of silane (Figure 4.11). The experiment was set-up by dissolving the Ru complex $(0.5 \mathrm{~mol} \%)$ in $\mathrm{MeOH}(1 \mathrm{~mL})$. Addition of dimethylphenyl silane produced the quantitative evolution of hydrogen in 30 seconds. When the generation of hydrogen was stopped, a second equivalent of dimethylphenyl silane was added and hydrogen was again generated in 30 seconds. The process could be repeated for four consecutive cycles without any significant decrease in activity. In the fifth injection the yield decreased at $75 \%$ yield and in the sixth a $36 \%$ yield is obtained even after long reaction times, most probably due to the accumulation of the silyl ether in the reaction media. This experiment suggests that complex $\mathbf{3 C}$ is stable towards successive addition of hydrosilane with no or poor degradation of the catalyst. 


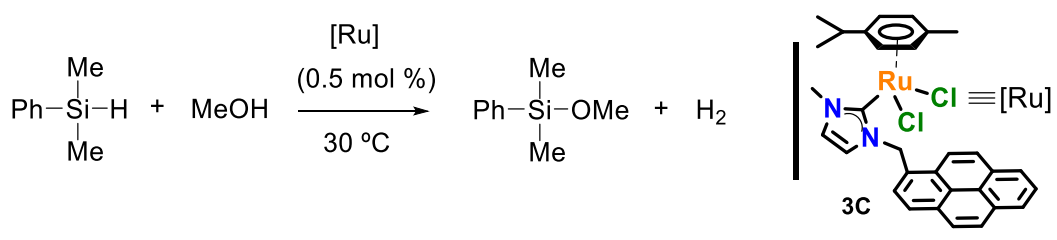

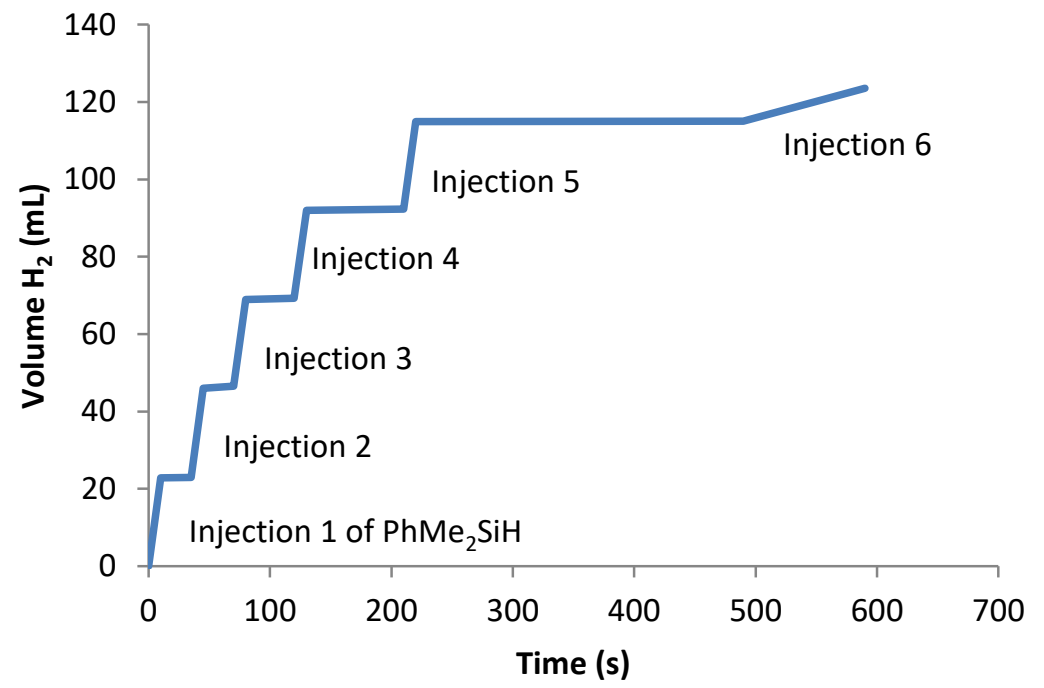

Figure 4.11 Time-profile for the reaction between dimethylphenyl silane and methanol obtained over six-additions of hydrosilane. Conditions: $1.0 \mathrm{mmol}$ of dimethylphenyl silane, $1 \mathrm{~mL}$ of $\mathrm{MeOH}$ and $3 \mathrm{mg}$ of $\mathbf{3 C}(0.5 \mathrm{~mol} \%)$. Yield corresponds to the amount of hydrogen collected using an inverted burette. An evolved volume of $24 \mathrm{~mL}$ corresponds to $100 \%$ yield

The stability of $\mathbf{3 C}$ was also tested in a large-scale experiment. At a low catalyst loading of $0.1 \mathrm{~mol} \%, 10 \mathrm{~g}$ of dimethylphenyl silane were converted into the corresponding silyl ether in quantitative yield at $30^{\circ} \mathrm{C}$ in 15 minutes. Removal of the solvent $(\mathrm{MeOH})$ afforded $12 \mathrm{~g}$ of the product in excellent purity. This result shows that $\mathbf{3 C}$ exhibits high stability and activity along with easy scalability.

The high stability of $\mathbf{3 C}$ is an excellent indication of the potential of the system to be recycled and used in following runs. However, separation of a homogeneous catalyst from the reaction media is a costly process. Alternatively, the use of heterogeneous catalysis facilitates the separation from the reaction media. Taking into account the results obtained in terms of recyclability with the grafted catalyst $\mathbf{3 C}$-rGO in the dehydrogenation of alcohols and amines, we decided to study the properties of 3C-rGO in the silane/alcohol coupling reaction. 
An initial experiment using only reduced graphene oxide shows that no activity could be attributed to the material itself, discarding the carbocatalytic scenario. Different hydrosilanes and alcohols can be reacted with 3C-rGO at a low catalyst loading of $0.05 \mathrm{~mol} \%$ (Table 4.4). In all experiments excellent yields of silyl ethers were obtained after filtration and solvent evaporation.

Table 4.4 Dehydrogenative coupling of silanes and alcohols using the Ru supported catalyst 3C-rGO

\begin{tabular}{cccccc}
\hline Entry & Catalyst & [Ru] (mol \%) & Silane & Alcohol & Yield (\%) \\
\hline 1 & rGO & - & $\mathrm{PhMe}_{2} \mathrm{SiH}$ & $\mathrm{MeOH}$ & 0 \\
2 & 3C-rGO & 0.05 & $\mathrm{PhMe}_{2} \mathrm{SiH}$ & $\mathrm{MeOH}$ & 100 \\
3 & 3C & 0.05 & $\mathrm{PhMe}_{2} \mathrm{SiH}$ & $\mathrm{MeOH}$ & 44 \\
4 & 3C-rGO & 0.05 & $\mathrm{PhMe}_{2} \mathrm{SiH}$ & $\mathrm{EtOH}$ & $100(93)$ \\
5 & 3C-rGO & 0.05 & $\mathrm{PhMe}_{2} \mathrm{SiH}$ & $n \mathrm{BuOH}$ & $95(90)$ \\
6 & 3C-rGO & 0.05 & $\mathrm{PhSiH}_{3}$ & $\mathrm{MeOH}$ & 90 \\
7 & 3C-rGO & 0.05 & $\mathrm{PhSiH}_{3}$ & $n \mathrm{BuOH}$ & $100(94)$ \\
\hline
\end{tabular}

Reaction conditions: Hydrosilane $(1.0 \mathrm{mmol})$, catalyst $3 \mathrm{C}$ or $\mathbf{3 C}-\mathrm{rGO}(0.05 \mathrm{~mol} \%$ based on $\mathrm{Ru}), 1 \mathrm{~mL}$ of $\mathrm{ROH}$ at $30{ }^{\circ} \mathrm{C}$ for $10 \mathrm{~min}$. [a] Isolated yields of silyl ether in parenthesis determined by ${ }^{1} \mathrm{H}$ NMR spectroscopic analysis using 1,3,5-trimethoxybenzene as external standard.

Analysis of the results contained in Table 4.4 show that the catalytic activity of the hybrid material $\mathbf{3 C}$-rGO is superior to the activity of $\mathbf{3 C}$ under the same reaction conditions (entries 2 and 3). Usually grafting molecular catalysts on a solid matrix cause a drop in the catalytic activity due to the more difficult diffusion process. However, graphene is a 2D flat material that allows the easy flow of the substrates to the catalytic centres. A plausible explanation for the increase in the activity is that the rGO material is somehow stabilising the molecular catalysts anchored in its surface, avoiding decomposition pathways of $\mathbf{3 C}$ and allowing carrying out the reaction at a lower catalyst loading.

The recyclability of $\mathbf{3 C - r G O}$ was evaluated using dimethylphenyl silane and methanol as model substrates. After each run the solid catalyst was separated by decantation, washed thoroughly with $\mathrm{MeOH}$, and used in the subsequent run. Using this methodology $\mathbf{3 C}$-rGO could be used in ten consecutive runs without any decrease in activity (Figure 4.12). 

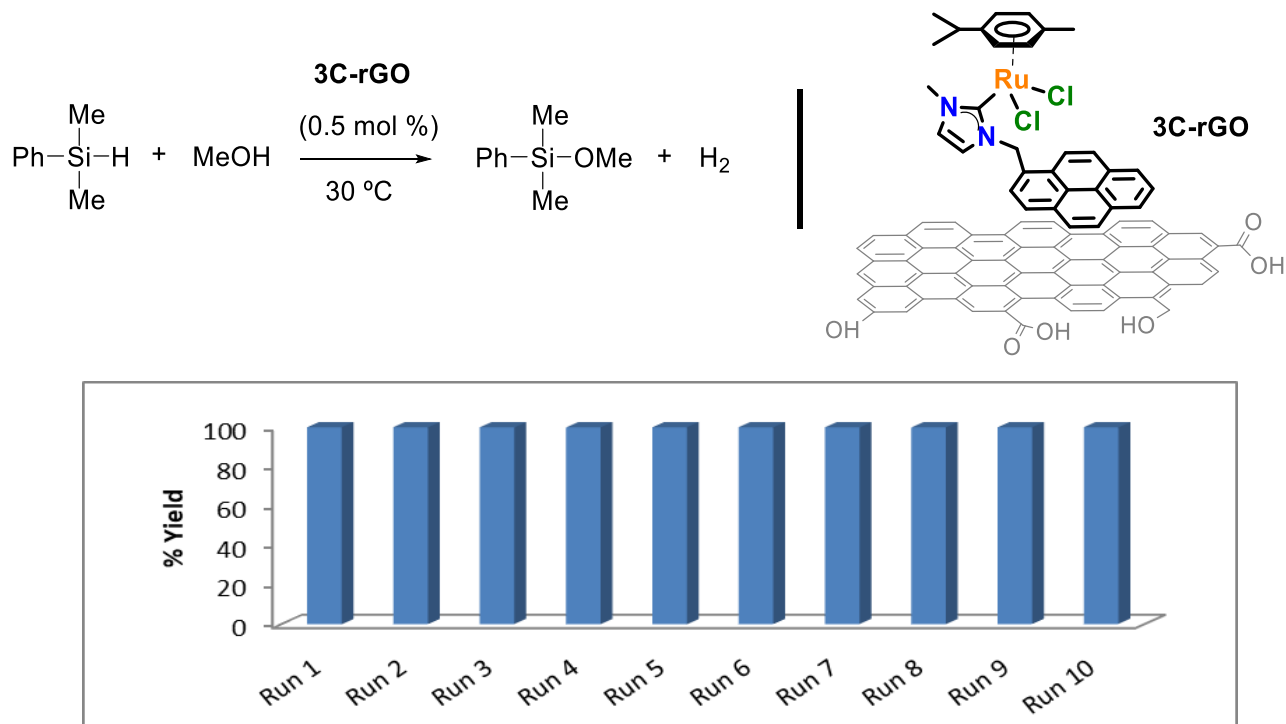

Figure 4.12 Recycling experiment using dimethylphenyl silane as model substrate. Reaction conditions: 3C-rGO $0.5 \mathrm{~mol} \%$ (based on Ru). In each run $\mathrm{Ph}(\mathrm{Me})_{2} \mathrm{SiH}(1.0 \mathrm{mmol}), 3 \mathrm{~mL}$ of $\mathrm{MeOH}$ at $30^{\circ} \mathrm{C}$ for $10 \mathrm{~min}$. Yields determined by GC using anisole as the standard

The results in terms of recyclability are excellent and compare or overcome the recycling properties of other heterogeneous catalysts based on $\mathrm{Cu}^{[88,89]}, \mathrm{Ag}^{[90]}$ or $A u{ }^{[91,92]}$ Deactivation of the catalyst is in part limited due to the soft conditions used during the catalysis. The mild reaction conditions avoid leaching of metal complex from the surface and do not affect the properties of the support.

After the recycling experiment the recovered solid catalyst 3C-rGO was analysed by TEM and XPS to study any possible change in the morphology or the structure. HRTEM images before and after 10 catalytic cycles shows that the morphology and the $2 \mathrm{D}$ nature of the graphene are preserved (Figure 4.13 , a and $\mathrm{b}$ ). The micrographs show the absence of metal nanoparticles that may be formed under a reductive atmosphere of hydrogen, suggesting that the nature of the catalytic species is molecular. Analysis by EDS (Figure 4.13, c) shows a homogeneous distribution of ruthenium over the entire surface. 


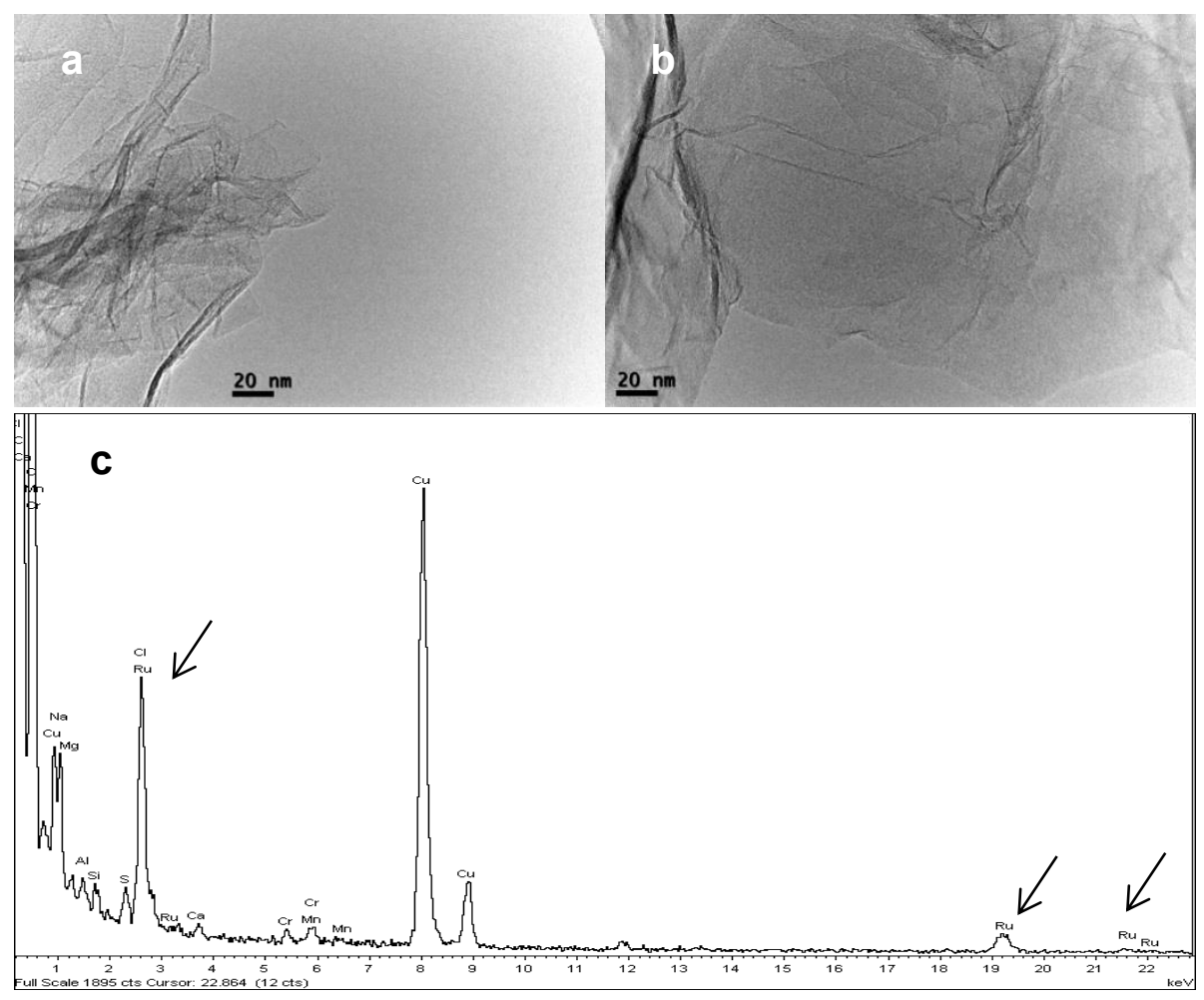

Figure 4.13 HRTEM images of 3C-rGO before (a) and after (b) ten catalytic runs and EDS spectrum (c) after ten runs showing the presence of Ru on the surface of rGO

Further evidence of the presence of $\mathbf{3 C}$ on graphene after the recycling experiment was obtained by XPS analysis (Figure 4.14). Comparison of the Ru3d, $\mathrm{Cl} 2 \mathrm{p}$ and N1s core-level peaks for the molecular complex $3 \mathrm{C}$ and the hybrid material $3 \mathrm{C}$-rGO before and after the catalysis show the same binding energy for the three different elements. These results reveal that the chemical environment around the $R u$ is maintained after the immobilisation onto $\mathrm{rGO}$ and after the catalysis. The XPS spectra confirm the presence of molecular complex $3 \mathbf{C}$ on the surface of rGO after the 10 catalytic runs. 

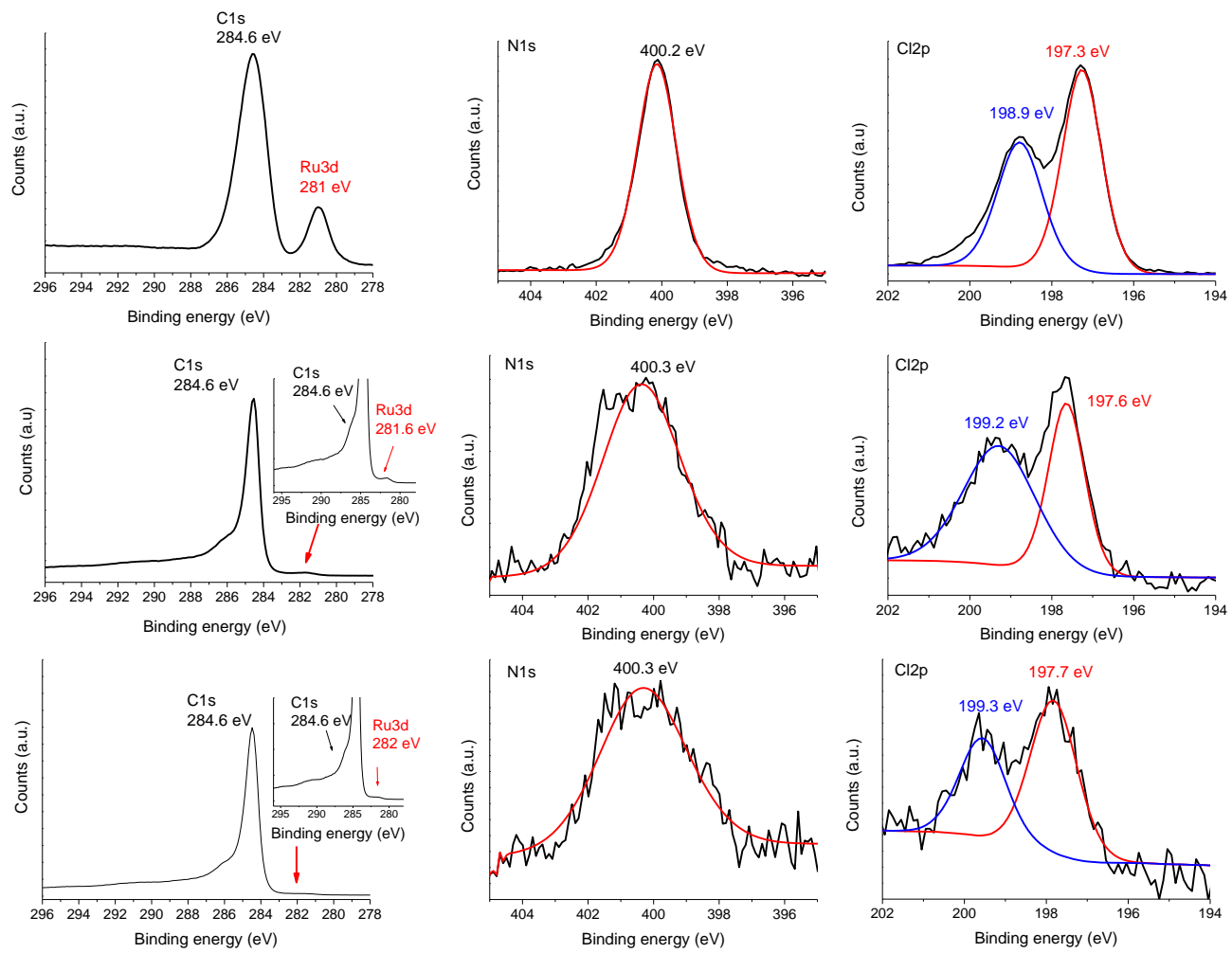

Figure 4.14 Comparative XPS analysis of 3C (top), 3C-rGO (middle), and 3C-rGO after 10 catalytic cycles (bottom) for the Ru3d, N1s and Cl2p core-level peaks

\subsubsection{Hydrogen production from the coupling between silanes and alcohols catalysed by iridium complexes anchored onto rGO}

To study the catalytic properties of complexes $\mathbf{8 C}$ and $\mathbf{8} \mathbf{C}^{\prime}$ dimethylphenyl silane and methanol were the initial substrates of choice. The reaction was set-up in a round botton flask and under air. Solvents were dried over pre-activated molecular sieves of $3 \AA$ A. A suitable amount of the iridium complex ( $0.5 \mathrm{~mol} \%$ respect the hydrosilane) was dissolved in methanol $(1 \mathrm{~mL})$. For practical purposes the alcohol acted as both, reagent and solvent. Then, the hydrosilane was added with the aid of a syringe and the reaction started. The activity of the ortometallated iridium complex $\mathbf{8} \mathbf{C}^{\prime}$ was almost negligible and it was not further investigated. On the contrary, when using $\mathbf{8 C}$ the reaction immediately started to generate hydrogen, the amount of which was recorded using either, an inverted burette or a pressure transducer. The reaction was finished within 2 minutes as evaluated using the inverted burette. After the reaction the formed product was isolated by simple evaporation of the methanol. Analysis by ${ }^{1} \mathrm{H}$ NMR showed an individual set of peaks that correspond with the 
formation of (methoxy)dimethylphenyl silane in quantitative yield. Drying the solvent over molecular sieves was essential. With technical $\mathrm{MeOH}$ a 7/3 mixture of the silyl ether and silanol products was observed. After drying the solvent, $>99 \%$ of the isolated product corresponded with the silyl ether.

Then, the reaction kinetics was evaluated using a pressure transducer. Comparison of the reaction profiles show that the reaction is slightly more rapid when using an inverted burette as the measurement system (Figure 4.15, a). This result can be attributed to the easier removal of the generated hydrogen gas when using an open system such as the inverted burette.

The dehydrogenative coupling of dimethylphenyl silane works with different primary aliphatic alcohols. The reaction kinetics is affected by the length of the aliphatic chain (Figure 4.15, b). The rates quickly decrease as the length of the aliphatic chain increases. For instance, the reaction is complete in 12 minutes using ethanol, but requires almost one hour to reach $100 \%$ yield when using butanol. This effect is more important when sterically more hindered secondary alcohols are used. In the case of 2-propanol the reaction is stopped after 80 minutes with a moderate conversion of $40 \%$.
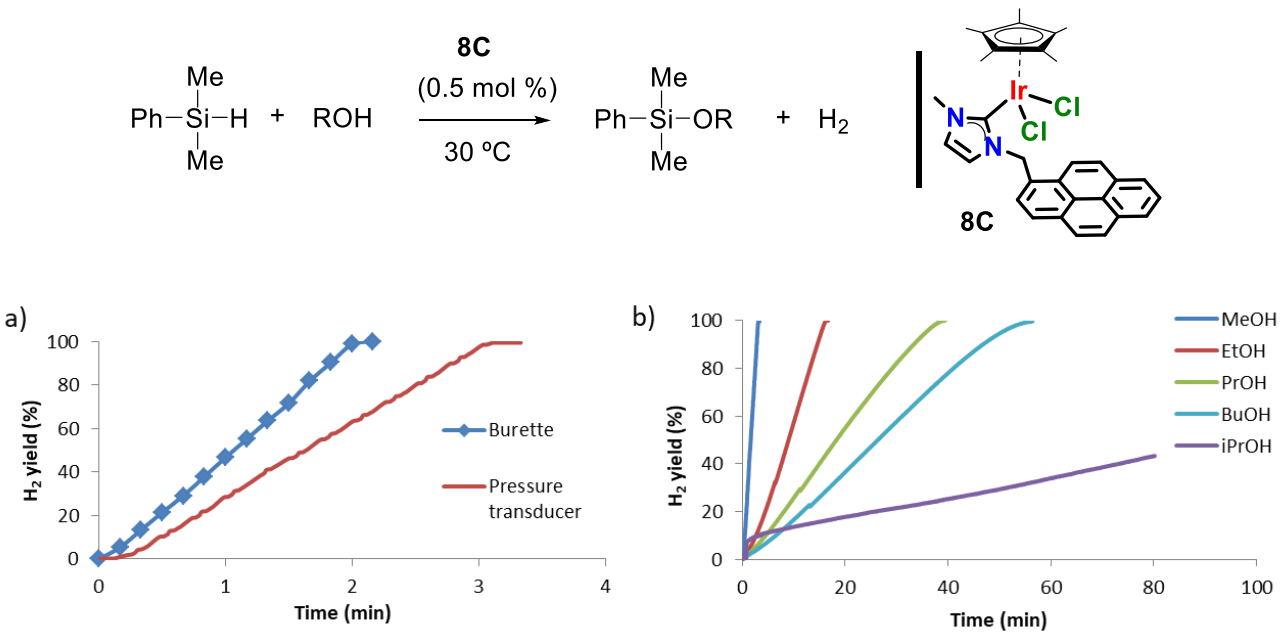

Figure 4.15 a) Hydrogen evolution monitoring for the reaction of dimethylphenyl silane with methanol at $30{ }^{\circ} \mathrm{C}$ using catalyst $8 \mathrm{C}(0.5 \mathrm{~mol} \%)$. b) Dehydrogenative coupling of dimethylphenyl silane with different alcohols using catalyst $8 \mathrm{C}(0.5 \mathrm{~mol} \%)$. Yields of hydrogen obtained using a pressure transducer 
The scope of the dehydrogenative coupling between alcohols and hydrosilanes catalysed by $\mathbf{8 C}$ was studied (Table 4.5 ). Tertiary silanes were converted to the corresponding silyl ethers in excellent yields at a low catalyst loading of $0.5 \mathrm{~mol} \%$. The system is sensitive to the alcohol sterics. The reaction time increased as the aliphatic chain of the alcohol increased (see entries $1-4$ and $5-6$ ). The reactions were very fast using $\mathrm{MeOH}$ as substrate, but the rate was slown down when using longer aliphatic alcohols. Bulkier hydrosilanes such as diphenyl silane showed slower reactivity. Yet, a good $82 \%$ isolated yield was obtained after two hours using methanol as substrate (entry 7).

Table 4.5 Scope of the dehydrogenative coupling of silanes and alcohols catalysed by $8 \mathrm{C}$

$$
\mathrm{R}_{4-n} \mathrm{SiH}_{n}+n \mathrm{R}^{\prime} \mathrm{OH} \frac{[\mathrm{lr}]}{30{ }^{\circ} \mathrm{C}} \mathrm{R}_{4-\mathrm{n}} \mathrm{Si}\left(\mathrm{OR}^{\prime}\right)_{n}+\mathrm{nH}_{2}
$$

\begin{tabular}{|c|c|c|c|c|c|}
\hline Entry & Silane & ROH & eff HSC (\%) & $t(\min )$ & Yield (\%) \\
\hline 1 & & $\mathrm{MeOH}$ & 1.2 & 3 & $100(98)$ \\
\hline 2 & & EtOH & 1.1 & 12 & $100(97)$ \\
\hline 3 & & $n \mathrm{PrOH}$ & 1.0 & 40 & $100(98)$ \\
\hline 4 & & $n \mathrm{BuOH}$ & 0.96 & 55 & 100 (99) \\
\hline 5 & \multirow{2}{*}{$\mathrm{Et}_{3} \mathrm{SiH}$} & $\mathrm{MeOH}$ & 1.4 & 20 & 100 \\
\hline 6 & & EtOH & 1.2 & 30 & 100 \\
\hline 7 & $\mathrm{Ph}_{2} \mathrm{SiH}_{2}$ & $\mathrm{MeOH}$ & 1.6 & 120 & $85(82)$ \\
\hline 8 & \multirow{2}{*}{$\square-\mathrm{SiH}_{3}$} & $\mathrm{MeOH}$ & 3.0 & 5 & 100 \\
\hline 9 & & $\mathrm{EtOH}$ & 2.5 & 15 & $100(94)$ \\
\hline 10 & & $\mathrm{MeOH}$ & 2.8 & 20 & $100(93)$ \\
\hline 11 & & $\mathrm{EtOH}$ & 2.3 & 35 & $100(90)$ \\
\hline 12 & \multirow[b]{2}{*}{$\sim^{\mathrm{SiH}_{3}}$} & $\mathrm{MeOH}$ & 3.3 & 15 & $100(75)$ \\
\hline 13 & & $\mathrm{EtOH}$ & 2.7 & 20 & $96(80)$ \\
\hline 14 & \multirow{4}{*}{$\mathrm{H}_{3} \mathrm{Si} \frown \mathrm{SiH}_{3}$} & $\mathrm{MeOH}$ & 4.3 & 12 & $100(96)$ \\
\hline $15^{\mathrm{b}}$ & & EtOH & 3.3 & 30 & $100(97)$ \\
\hline $16^{c}$ & & $n \mathrm{PrOH}$ & 2.7 & 25 & 100 \\
\hline $17^{c}$ & & $n \mathrm{BuOH}$ & 2.3 & 40 & 100 \\
\hline
\end{tabular}

Reaction conditions: Hydrosilane $(1.0 \mathrm{mmol})$, iridium catalyst $8 \mathrm{C}(0.5 \mathrm{~mol} \%), 30{ }^{\circ} \mathrm{C}$ and $1 \mathrm{~mL}$ of ROH. [a] Yields determined by $\mathrm{H}_{2}$ formation using an inverted burette and/or a pressure transducer. Isolated yields in parenthesis based on the amount of silyl ether determined by ${ }^{1} \mathrm{H}$ NMR spectroscopic analysis using 1,3,5-trimethoxy benzene as an external standard. [b] Iridium catalyst (1 mol \%). [c] Iridium catalyst (2 mol \%). 
The effective HSC of the silane/alcohol pair strongly depends on structure of the reactants. The HSC of hydrosilanes containing a single $\mathrm{Si}-\mathrm{H}$ bond is low. For example the $\mathrm{PhMe}_{2} \mathrm{SiH} / \mathrm{MeOH}$ pair has a 1.2 wt \% of hydrogen. Moving to a secondary hydrosilane such as $\mathrm{Ph}_{2} \mathrm{SiH}_{2}$ increases the $\mathrm{HSC}$ to $1.6 \mathrm{wt} \%$. The US energy department has set the gravimetric target of a LOHC for on-board use in vehicles in 5.5 wt \%. ${ }^{[6]}$ Thus, more hydrogen rich silanes are needed. The structural versatility of organosilanes allows the increase of the HSC when using primary silanes and disilanes. The pairs phenylsilane/methanol (HSC = $3 \mathrm{wt} \%$ ), tolylsilane/methanol ( $\mathrm{HSC}=2.8 \% \mathrm{wt}$ ) and butylsilane/methanol (HSC = 3.3 wt \%) are reacted at room temperature using a catalyst loading of $0.5 \mathrm{~mol} \%$ and giving the corresponding silyl ethers and hydrogen in excellent yields (entries 8,10 and 12). The best result in terms of hydrogen capacity is obtained using 1,4 disilabutane/MeOH which generate $6 \mathrm{~mol}$ of hydrogen per mol of hydrosilane (entry 14). The hydrogen content of this pair is $4.3 \mathrm{wt} \%$, very close to the HSC of formic acid. However, the use of 1,4 disilabutane/MeOH shows a big advantage, because the formation of the greenhouse gas $\mathrm{CO}_{2}$ is avoided.

\section{Recyclability studies with 8 C-rGO and catalyst stability}

The immobilisation of complex $\mathbf{8 C}$ onto rGO (Figure 4.8) leads to a solid material that can act as a potential and recyclable hybrid catalyst. The catalytic properties of 8C-rGO were tested in the dehydrogenative coupling between dimethylphenyl silane and $\mathrm{MeOH}$. Using a catalyst loading of $0.5 \mathrm{~mol} \%$ (based on Ir) the hybrid material 8C-rGO accounts for a $100 \%$ yield after 7 minutes. As observed for catalysts $\mathbf{3 C}$ and 3C-rGO, the fact of grafting molecular $\mathbf{8 C}$ on graphene do not have a critical effect on the reaction and the catalytic activity at the molecular level (100\% in 3 minutes using $\mathbf{3 C}$ ) compare to the activity using $\mathbf{8 C}-\mathbf{r G O}$ (100\% in 7 minutes).

Further evidence of the high stability of $\mathbf{8 C}-$ rGO was obtained by lowering the amount of catalyst. At a catalyst loading of $0.1 \mathrm{~mol} \%$ the homogeneous complex $8 \mathrm{C}$ accounted for a $70 \%$ conversion at $30{ }^{\circ} \mathrm{C}$ after 15 minutes. On the contrary, a $100 \%$ conversion was obtained with $\mathbf{8 C}$-rGO under identical reaction conditions. This result suggests that graphene is not acting as a mere support and it is playing a role in the catalysis. A plausible explanation for this effect is that $\mathrm{rGO}$ is somehow limiting the degradation processes and/or stabilising the catalytically active species.

The recycling properties of $\mathbf{8 C}$-rGO were tested with the pair dimethyphenyl silane/methanol at $30{ }^{\circ} \mathrm{C}$, using a catalyst loading of $0.5 \mathrm{~mol} \%$. The mixture was 
allowed to react for 10 minutes. After each run, the solid catalyst was separated from the reaction media by decantation, washed with methanol and reused in the following reaction. Using this methodology, $\mathbf{8 C}-\mathbf{r G O}$ could be recycled up to 10 times without any observed decrease in activity (Figure 4.16). This result shows that grafting $\mathbf{8 C}$ on the surface of rGO leads to the formation of a highly robust and recyclable hybrid material.

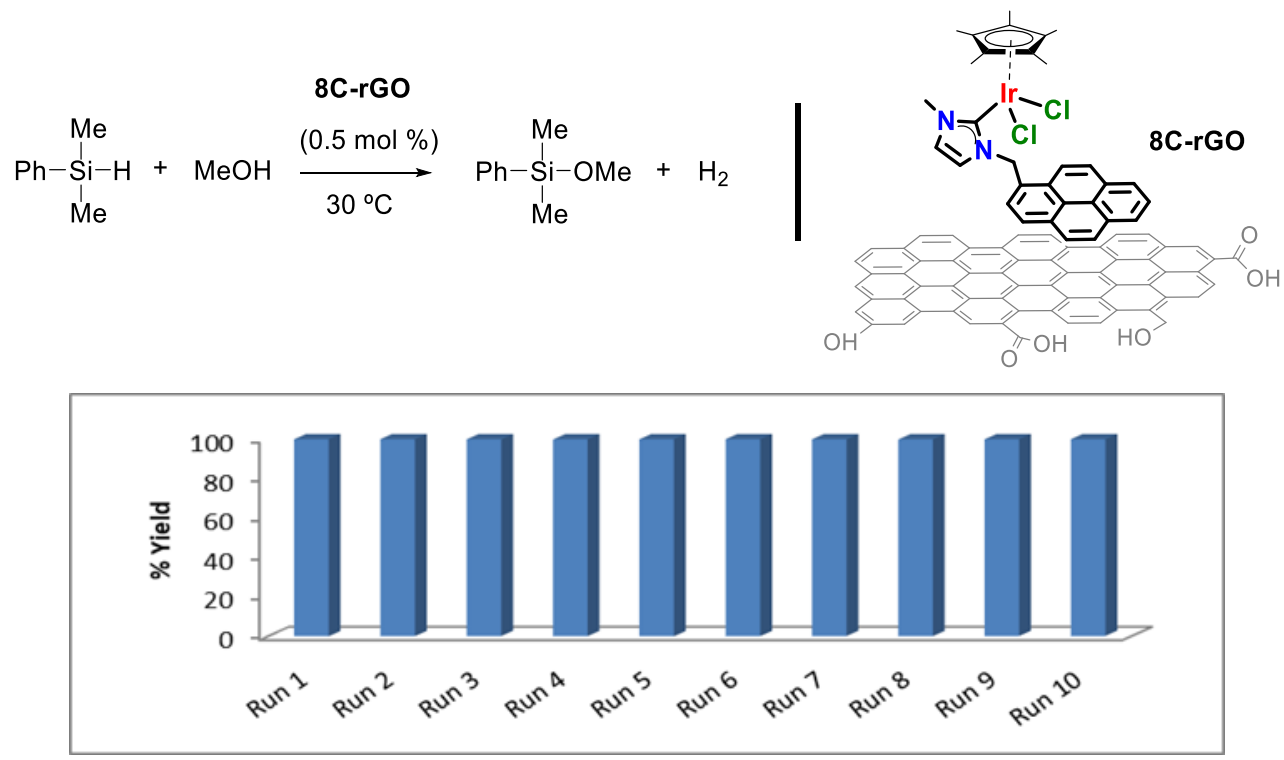

Figure 4.16 Recycling experiment using dimethylphenyl silane as model substrate. Reaction conditions: 8C-rGO $0.5 \mathrm{~mol} \%$ (based on $\mathrm{Ir}$ ). In each run $\mathrm{Ph}(\mathrm{Me}){ }_{2} \mathrm{SiH}(1.0 \mathrm{mmol}), 3 \mathrm{~mL}$ of $\mathrm{MeOH}$ at $30^{\circ} \mathrm{C}$ for $10 \mathrm{~min}$. Yields determined by GC using anisole as the standard

After the recycling experiment the material was analysed by HRTEM. The micrographs revealed that the morphology of the graphene maintains its monolayer structure, albeit with the presence of more wrinkles. Interestingly, the EDS spectrum showed a homogeneous distribution of iridium and no NPs were generated, which is an indication of the molecular nature of the catalytic active species.

\subsubsection{Mechanistic studies}

The mechanism for the catalytic dehydrogenative coupling between hydrosilanes and alcohols was studied for both, the ruthenium and the Iridium catalysts. ${ }^{[93,94]}$ The results show two very similar mechanistic cycles and reaction profiles, with the same elementary steps and very close energy values for each step. In consequence, only the Ru-catalysed version is described in this section. A plausible mechanistic cycle is shown in Scheme 4.2. 
The mechanistic proposal is based on the ionic mechanism proposed by Crabtree et al., which suggests the interaction of the $\sigma \mathrm{Si}-\mathrm{H}$ bond with the metal complex as the first step. ${ }^{[75]}$ The formation of a Ru $\sigma$-Silane complex requires the generation of a vacant site, which comes from the dissociation of one chloride ligand. Halide dissociation has been previously studied for $\mathrm{Ru}$ complexes and depends on the solvent, $\mathrm{pH}$ and the presence of nucleophiles. ${ }^{[95]}$ To confirm the ligand dissociation, complex 7G was analysed by ${ }^{1} \mathrm{H}$ NMR using $\mathrm{CDCl}_{3}$ as solvent. The spectrum shows the presence of one sharp set of signals. On the contrary, when $\mathrm{CDCl}_{3}$ was removed and 7G was dissolved in $\mathrm{CD}_{3} \mathrm{OD}$, the signals broadened and different species could be identified, but its exact nature could not be unambiguously assigned. Moving back to $\mathrm{CDCl}_{3}$ as solvent restores the initial situation, showing a reversible process. These results support that methanol promotes the formation of cationic species of general formula $[\mathrm{Ru}(p-\mathrm{cym})(\mathrm{NHC}) \mathrm{Cl}(\mathrm{MeOH})]^{+}$(i-MeOH in Scheme 4.2).

The formation of $\mathbf{i}-\mathbf{M e O H}$, allows the interaction of the metal centre with the hydrosilane and the formation of the $\sigma$-silane complex I by displacement of the labile methanol ligand. Alternatively, the silane could be oxidatively added to the Ru metal centre generating a Ru (IV) hydride complex (Figure 4.17). DFT calculations performed by Dr. Miguel Baya shows that the oxidative addition is a highly disfavoured process $(\Delta G=21.2 \mathrm{kcal} / \mathrm{mol}$ respect the formation of the $\sigma$ complex $)$ and rule out the formation of Ru (IV) species (Figure 4.17). Analysis of the interaction between the hydrosilane and the methanol adduct also reveal that $\eta^{1}$ is a more favoured coordination mode than $\eta^{2}$. The origin of this preferred $\eta^{1}-\mathrm{H}-\mathrm{SiR}_{3}$ coordination is attributed to steric factors, as has been observed for bulky iridium complexes. ${ }^{[96]}$

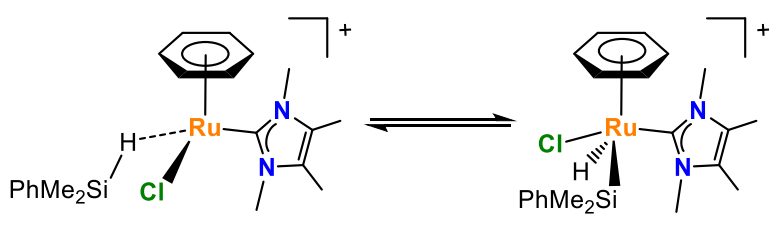

$\Delta \mathbf{G}^{\circ}=+21.2 \mathrm{kcal} / \mathrm{mol}$

Figure 4.17 Behaviour of 7G towards dimethylphenyl silane

The interaction between the $\mathrm{Ru}$ centre and the hydrosilane generates the intermediate I. The electrophilicity of the coordinated silane is boosted, making the silicon atom more susceptible from a nucleophilic attack by $\mathrm{MeOH}$. The computational model suggest a back attack of the methanol to the silicon, as 
previously observed in CpFe compounds. ${ }^{[97]}$ In this step (TS1), the Si-O and Ru-H bonds are forming while the $\mathrm{Si}-\mathrm{H}$ bond is breaking (Scheme 4.2). As a consequence, a metal hydride (III) is formed along with a $\left[\mathrm{R}_{3} \mathrm{Si}(\mathrm{H}) \mathrm{OMe}\right]^{+}$cation. DFT calculations suggest that the alcohol nucleophilic attack is the rate determining step of the reaction, with an energy barrier of $8 \mathrm{Kcal} / \mathrm{mol}$. The low energy barrier is in accordance with the soft conditions experimentally used. In spite of this apparently barrierless process, a ruthenium catalyst is needed to make the reaction run (Table 4.2 , entry 1 ). In the presence of the Ru catalyst, the activation energy is low and the reaction works at low temperatures (even $-25^{\circ} \mathrm{C}$ ) and in short times (50 s). The initial rates are very fast, indicating that a limited energy input is needed to start the reaction. Another experimental observation that supports that alcohol nucheophilic attack is involved in the rate determining step is the dependence of the reaction on the nucleophilicity of the alcohols (Table 4.3, entries 3-12). The reactions perform better when the nucleophilicity of alcohol is increased, thus suggesting that alcohol attack to the coordinated silane is a key step in the catalytic cycle.

The cation formed after the alcohol attack subsequently protonates the hydride, generating the dihydrogen species $\mathbf{V}$ and the silyl ether product (Scheme 4.2). The last step comprises the release of molecular hydrogen and the coordination of a new silane molecule, regenerating I and closing the loop. 

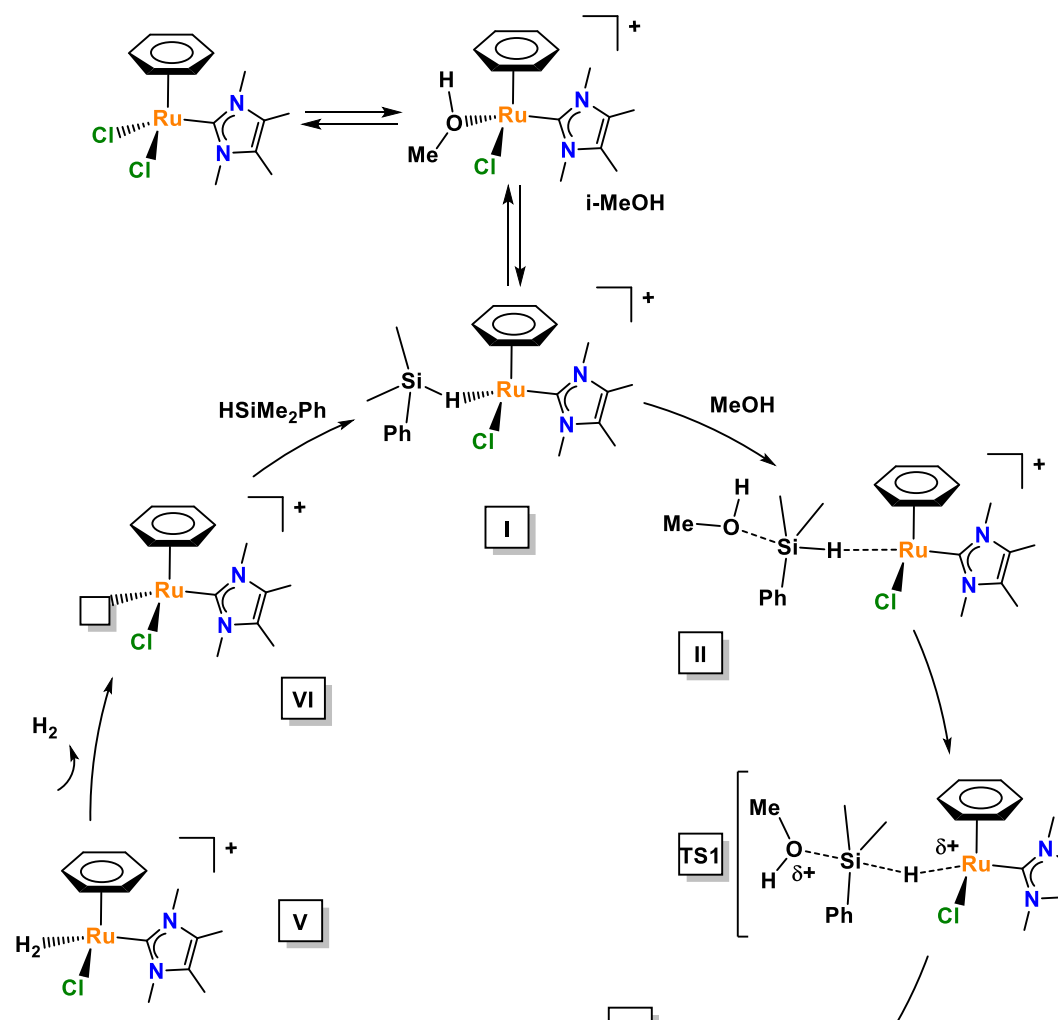

VI
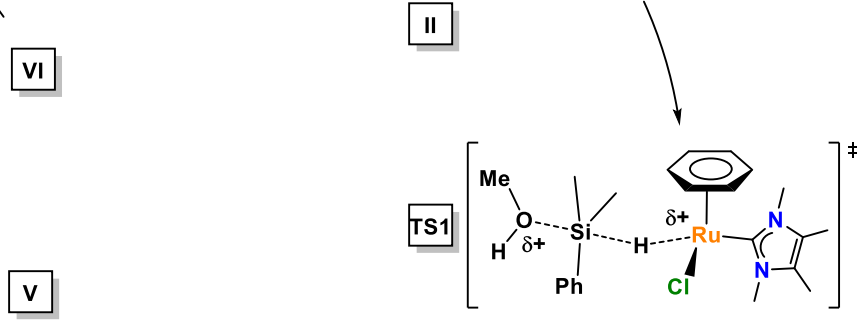<smiles>CO[Si](C)(C)c1ccccc1</smiles><smiles>CO[Si](C)(C)c1ccccc1</smiles>

III
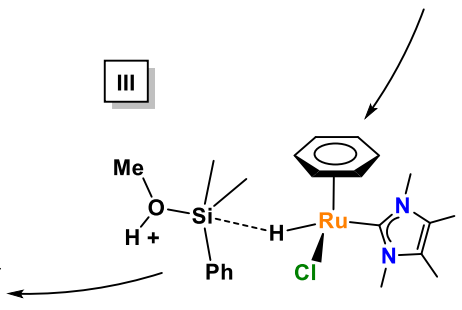

Scheme 4.2 Mechanistic proposal for the dehydrogenative coupling of dimethylphenylsilane and methanol catalysed by $\left[\mathrm{Ru}\left(\eta^{6}-\mathrm{C}_{6} \mathrm{H}_{6}\right) \mathrm{Cl}_{2}(\mathrm{NHC})\right]$

An important consideration to take into account is the inherent reactivity between silanes and Ru-NHC complexes (Figure 4.18). Prof. D. Stephan and co-corkers reported that treatment of a Ru-NHC complex with one equivalent of a tertiary silane in dichloromethane affords a silyl chloride and a Ru-H complex. ${ }^{[98]}$ In our case the silyl chloride was not observed at the end of the reaction because of the critical effect of the solvent. In alcoholic media the silyl chloride that may form would quickly react with $\mathrm{ROH}$ to obtain a silyl ether and hydrogen chloride $(\mathrm{HCl})$. The $\mathrm{HCl}$ immediately protonates the ruthenium hydride which then release hydrogen gas. 
As a result, silyl chlorides are not observed. The ruthenium-hydride species takes part in the cycle, but its half-life in acidic media is too short and therefore it is not observed experimentally.
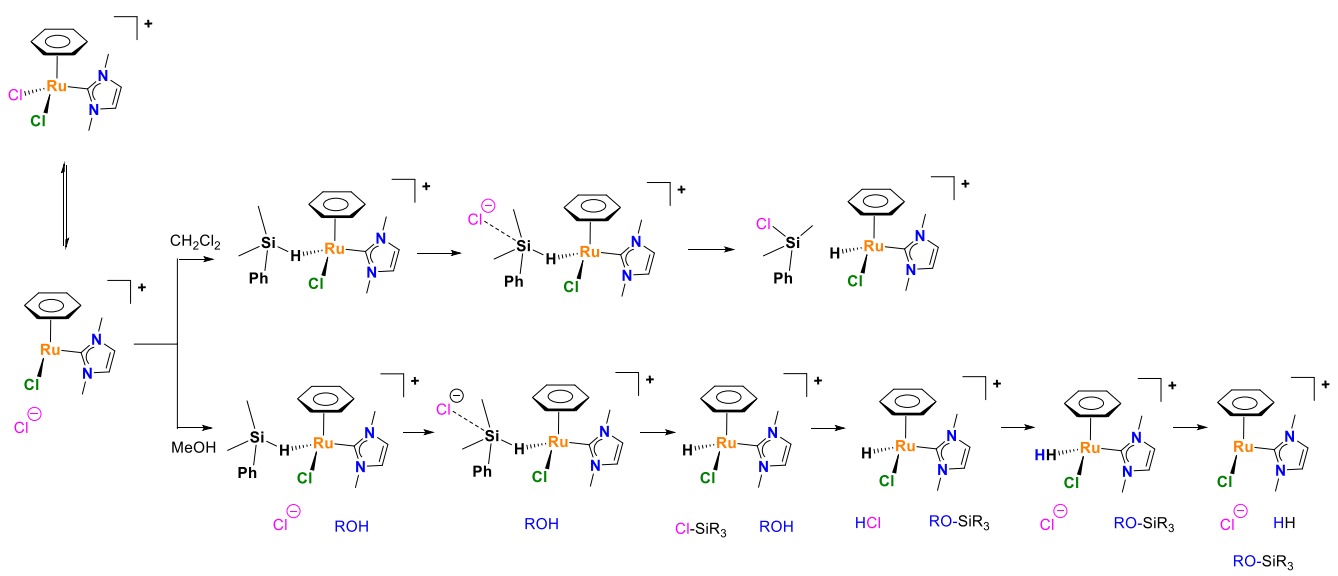

Figure 4.18 Different reactivity of Ru-NHC complexes with silanes in $\mathrm{CH}_{2} \mathrm{Cl}_{2}$ or $\mathrm{MeOH}$ 


\subsection{CONCLUSIONS}

The preparation of a new iridium complex with an NHC ligand that features a pyrene tag is described in this chapter. The reaction conditions during the synthesis determine if the isolated product is orthometalated $\left(\mathbf{8} C^{\prime}\right)$ or not $(\mathbf{8 C})$. Avoiding orthometalation is key, as the catalytic properties of $\mathbf{8 C}$ and $\mathbf{8 \mathbf { C } ^ { \prime }}$ are completely different. $\mathbf{8 C}$ can be grafted onto graphene under soft reaction conditions affording the new material $\mathbf{8 C}$-rGO, which has been fully characterised by HRTEM, XPS, UV/Vis and ICP-MS.

The catalytic properties of the homogeneous complexes $3 \mathrm{C}(\mathrm{Ru})$ and $8 \mathrm{C}(\mathrm{Ir})$ and the related hybrid materials $\mathbf{3 C}-\mathbf{r G O}$ and $\mathbf{8 C}-\mathbf{r G O}$ has been evaluated in the generation of hydrogen from the coupling between silanes and alcohols. The results show that both complexes are active in carrying out this transformation at low catalyst loading (0.1 - $0.5 \mathrm{~mol} \%)$. The reaction proceeds low temperature $\left(-25\right.$ to $30{ }^{\circ} \mathrm{C}$ ) and hydrogen is generated at fast rates and with complete selectivity towards the formation of silyl ether.

Mechanistic studies reveal a virtual barrier-less process promoted by $\mathrm{Ir}$ and $\mathrm{Ru}$ complexes, where the alcohol nucleophilic attack is the rate determining step. In the absence of catalyst the reaction do not proceed, but when $\mathbf{3 C}$ or $\mathbf{8 C}$ are in the media the reaction need a low energy input and takes place at fast rates.

The hybrids materials $\mathbf{3 C}-\mathbf{r G O}$ and $\mathbf{8 C}-\mathbf{r G O}$ are excellent catalysts for this reaction in terms of recyclability. Both materials can be reused up to ten times without any apparent decrease in activity. Analysis of the solid catalysts after the recycling experiment shows the absence of metal nanoparticles, indicating the molecular nature of the active species. In addition, the reduced graphene oxide is not a mere support. Anchoring homogeneous complexes onto rGO permits to carry out the reaction using lower catalyst loadings, showing that graphene is stabilising the active species. 


\subsection{REFERENCES}

[1] N. Armaroli, V. Balzani, Energy for a Sustainable World, Wiley-VCH Verlag GmbH \& Co. KGaA, Weinheim, Germany, 2010.

[2] V. Blagojevic', D. Minić, J. G. Novakovic', D. Minic', in Hydrogen Energy Challenges and Perspectives, InTech, London, 2012, pp. 3-28.

[3] G. W. Crabtree, M. S. Dresselhaus, M. V Buchanan, G. W. Crabtree, M. S. Dresselhaus, M. V Buchanan, Phys. Today 2004, 57, 1625-1637.

[4] G. Marbán, T. Valdés-solís, Int. J. Hydrogen Energy 2007, 32, 1625-1637.

[5] M. Markiewicz, Y. Q. Zhang, A. Bösmann, N. Brückner, J. Thöming, P. Wasserscheid, S. Stolte, Energy Environ. Sci. 2015, 8, 1035-1045.

[6] K. Sordakis, C. Tang, L. K. Vogt, H. Junge, P. J. Dyson, M. Beller, G. Laurenczy, Chem. Rev. 2018, 118, 372-433.

[7] A. P. Simpson, A. E. Lutz, Int. J. Hydrogen Energy 2007, 32, 4811-4820.

[8] J. Dufour, D. P. Serrano, J. L. Gálvez, J. Moreno, C. García, Int. J. Hydrogen Energy 2009, 34, 1370-1376.

[9] M. Balat, Int. J. Hydrogen Energy 2008, 33, 4013-4029.

[10] E. Gianotti, M. Taillades-jacquin, J. Roziere, D. J. Jones, ACS Catal. 2018, 8, 4660-4680.

[11] P. Preuster, C. Papp, P. Wasserscheid, Acc. Chem. Res. 2017, 50, 74-85.

[12] D. Teichmann, W. Arlt, P. Wasserscheid, R. Freymann, Energy Environ. Sci. 2011, 4, 2767-2773.

[13] M. Eypasch, M. Schimpe, A. Kanwar, T. Hartmann, S. Herzog, T. Frank, T. Hamacher, Appl. Energy 2017, 185, 320-330.

[14] M. Taube, D. W. T. Rippin, D. L. Cresswell, W. Kneght, Int. J. Hydrogen Energy 1983, 8, 213-225.

[15] T. Schildhauer, E. Newson, S. Müller, J. Catal. 2001, 358, 355-358.

[16] P. M. Modisha, C. N. M. Ouma, R. Garidzirai, P. Wasserscheid, D. Bessarabov, Energy Fuels 2019, 33, 2778-2796.

[17] G. Cacciola, N. Giordano, G. Restuccia, Int. J. Hydrogen Energy 1984, 9, 411419. 
[18] D. Sebastián, C. Alegre, L. Calvillo, M. Pérez, R. Moliner, M. J. Lázaro, Int. J. Hydrogen Energy 2014, 39, 4109-4115.

[19] Y. Suttisawat, H. Sakai, M. Abe, P. Rangsunvigit, S. Horikoshi, Int. J. Hydrogen Energy 2011, 37, 3242-3250.

[20] G. Lee, Y. Jeong, B. Kim, J. Sae Han, H. Jeong, H. Bin Na, J. Chul Jung, Catal. commun. 2015, 67, 40-44.

[21] "Hydrogenious Technologies GmbH, Erlangen, Germany.," can be found under https://www.hydrogenious.net, access date 07/02/2020.

[22] G. P. Pez, A. R. Scott, C. A. C., H. Cheng, L. D. Bagzis, J. B. Appleby, Hydrogen Storage by Reversible Hydrogenation of Pi-Conjugated Substrates, 2003, U.S. Patent Appl. US7101530B2.

[23] A. Moores, M. Poyatos, Y. Luo, R. H. Crabtree, New J. Chem. 2006, 30, 16751678.

[24] E. Clot, O. Eisenstein, R. H. Crabtree, Chem. Commun. 2007, 22, 2231-2233.

[25] K. Stark, P. Keil, S. Schug, K. Mu, P. Wasserscheid, W. Arlt, J. Chem. Eng. Data 2016, 61, 1441-1448.

[26] M. Grellier, S. Sabo-Etienne, Dalton Trans. 2014, 43, 6283-6286.

[27] T. Li, I. Bergner, F. N. Haque, M. Z. Iuliis, D. Song, R. H. Morris, R. V August, Organometallics 2007, 26, 5940-5949.

[28] R. Reguillo, M. Grellier, N. Vautravers, L. Vendier, S. Sabo-Etienne, J. Am. Chem. Soc. 2010, 132, 7854-7855.

[29] K. Tokmic, B. J. Jackson, A. Salazar, T. J. Woods, A. R. Fout, J. Am. Chem. Soc. 2017, 139, 13554-13561.

[30] K. N. T. Tseng, A. M. Rizzi, N. K. Szymczak, J. Am. Chem. Soc. 2013, 135, 16352-16355.

[31] I. Dutta, S. Yadav, A. Sarbajna, S. De, M. Hölscher, W. Leitner, J. K. Bera, J. Am. Chem. Soc. 2018, 140, 8662-8666.

[32] T. Achard, J. Egly, M. Sigrist, A. Maisse-François, S. Bellemin-Laponnaz, Chem. - A Eur. J. 2019, 25, 13271-13274.

[33] K. Räuchle, L. Plass, H. J. Wernicke, M. Bertau, Energy Technol. 2016, 4, 193200. 
[34] T. Schaub, Phys. Sci. Rev. 2018, 3, 35-56.

[35] M. Nielsen, E. Alberico, W. Baumann, H.-J. Drexler, H. Junge, S. Gladialu, M. Beller, Nature 2013, 495, 85-89.

[36] R. S. Coffey, Chem. Commun. 1967, 923-924.

[37] B. Loges, A. Boddien, H. Junge, M. Beller, Angew. Chemie - Int. Ed. 2008, 47, 3962-3965.

[38] C. Fellay, P. J. Dyson, G. Laurenczy, Angew. Chemie - Int. Ed. 2008, 47, 39663968.

[39] R. E. Rodríguez-Lugo, M. Trincado, M. Vogt, F. Tewes, G. Santiso-Quinones, H. Grützmacher, Nat. Chem. 2013, 5, 342-347.

[40] "team FAST - Building the world's first bus on formic acid," can be found under http://www.teamfast.nl/, access date 07/02/2020.

[41] W. Leitner, E. Dinjus, F. Gaßner, J. Organomet. Chem. 1994, 475, 257-266.

[42] J. Tsai, K. M. Nicholas, J. Am. Chem. Soc. 1992, 114, 5117-5124.

[43] S. Moret, P. J. Dyson, G. Laurenczy, Nat. Commun. 2014, 5, 4017.

[44] S. Lu, Z. Wang, J. Li, J. Xiao, C. Li, Green Chem. 2016, 18, 4553-4558.

[45] A. Weilhard, M. I. Qadir, V. Sans, J. Dupont, ACS Catal. 2018, 8, 1628-1634.

[46] K. Müller, K. Stark, B. Müller, W. Arlt, Energy Fuels 2012, 26, 3691-3696.

[47] G. Z. Wang, J. E. Bäckvall, J. Chem. Soc. Chem. Commun. 1992, 337-339.

[48] N. Blaquiere, S. Diallo-garcia, S. I. Gorelsky, D. A. Black, K. Fagnou, J. Am. Chem. Soc. 2008, 8, 14034-14035.

[49] W. Zhan, Q. Zhu, Q. Xu, ACS Catal. 2016, 6, 6892-6905.

[50] D. Sutton, Andrew, A. K. Burrell, D. A. Dixon, E. B. Garner III, J. C. Gordon, T. Nakagawa, K. C. Ott, J. P. Robinson, M. Vasiliu, Science 2011, 331, 1426-1430.

[51] G. Wolf, J. Baumann, F. Baitalow, F. P. Hoffmann, Thermochim. Acta 2000, 343, 19-25.

[52] K. Kuciński, G. Hreczycho, ChemCatChem 2017, 9, 1868-1885. 
[53] F. Hoffmann, M. Cornelius, J. Morell, M. Fröba, Angew. Chemie - Int. Ed. 2006, 45, 3216-3251.

[54] G. Hreczycho, E. Frydrych-Tomczak, K. Sterzynska, J. Budna, A. Malinska, H. Maciejewski, M. Zabel, Appl. Organomet. Chem. 2015, 29, 216-220.

[55] H. Freiser, M. V. Eagle, J. Speier, J. Am. Chem. Soc. 1953, 75, 2824-2827.

[56] J. M. Brunel, Int. J. Hydrogen Energy 2010, 35, 3401-3405.

[57] W. Han, T. Kim, S. Kim, Y. Kim, Y. Kim, S. Nam, S.-O. Kang, Int. J. Hydrogen Energy 2011, 36, 12305-12312.

[58] A. A. Toutov, K. N. Betz, M. C. Haibach, A. M. Romine, R. H. Grubbs, Org. Lett. 2016, 18, 5776-5779.

[59] A. Grajewska, M. Oestreich, Synlett 2010, 2, 2482-2484.

[60] A. Weickgenannt, M. Oestreich, Chem. - An Asian J. 2009, 4, 406-410.

[61] J. M. Blackwell, K. L. Foster, V. H. Beck, W. E. Piers, J. Org. Chem. 1999, 64, 4887-4892.

[62] J. Cella, S. Rubinsztajn, Macromolecules 2008, 41, 6965-6971.

[63] C. N. Scott, C. S. Wilcox, J. Org. Chem. 2010, 75, 253-256.

[64] S. Vijjamarri, V. K. Chidara, J. Rousova, G. Du, Catal. Sci. Technol. 2016, 6, 3886-3892.

[65] J. M. S. Cardoso, R. Lopes, B. Royo, J. Organomet. Chem. 2015, 775, 173-177.

[66] S. Rommel, L. Hettmanczyk, J. E. M. N. Klein, B. Plietker, Chem. - An Asian J. 2014, 9, 2140-2147.

[67] K. Fukumoto, M. Kasa, H. Nakazawa, Inorganica Chim. Acta 2015, 431, 219221.

[68] H. Ito, A. Watanabe, M. Sawamura, Org. Lett. 2005, 7, 1869-1871.

[69] J. Ohshita, R. Taketsugu, Y. Nakahara, A. Kunai, 2004, 689, 3258-3264.

[70] D. Mukherjee, R. R. Thompson, A. Ellern, A. D. Sadow, ACS Catal. 2011, 1, 698-702.

[71] W. Sattler, G. Parkin, J. Am. Chem. Soc. 2012, 134, 17462-17465. 
[72] M. Mirza-Aghayan, R. Boukherroub, M. Bolourtchian, J. Organomet. Chem. 2005, 690, 2372-2375.

[73] R. L. Miller, S. V Maifeld, D. Lee, Org. Lett. 2004, 6, 2773-2776.

[74] Y. Ojima, K. Yamaguchi, N. Mizuno, Adv. Synth. Catal. 2009, 351, 1405-1411.

[75] X. L. Luo, R. H. Crabtree, J. Am. Chem. Soc. 1989, 111, 2527-2535.

[76] M. Aliaga-Lavrijsen, M. Iglesias, A. Cebollada, K. Garcés, N. García, P. J. Sanz Miguel, F. J. Fernández-Alvarez, J. J. Pérez-Torrente, L. A. Oro, Organometallics 2015, 34, 2378-2385.

[77] A. Biffis, M. Basato, M. Brichese, L. Ronconi, C. Tubaro, A. Zanella, C. Graiff, A. Tiripicchio, Adv. Synth. Catal. 2007, 349, 2485-2492.

[78] H. Ito, T. Saito, T. Miyahara, C. Zhong, M. Sawamura, Organometallics 2009, $28,4829-4840$.

[79] G. L. Larson, Y. Pan, B. C. Arkles, Low Molecular Weight Carbosilanes Precursors Thereof, and Methods of Preparation, 2013, U.S. Patent Appl US2013/274497.

[80] K. Aoyagi, K. Matsumoto, S. Shimada, K. Sato, Y. Nakajima, Organometallics 2019, 38, 210-212.

[81] K. Aoyagi, Y. Ohmori, K. Matsumoto, S. Shimada, K. Sato, Y. Nakajima, Chem. Commun. 2019, 55, 5859-5862.

[82] A. Boudin, G. Cerveau, C. Chuit, R. J. P. Corriu, C. Reye, Bull Chem. Soc. Jpn 1988, 61, 101-106.

[83] D. Tsushima, M. Igarashi, K. Sato, S. Shimada, Chem. Lett. 2017, 46, 15321534.

[84] T. Beppu, K. Sakamoto, Y. Nakajima, K. Matsumoto, K. Sato, S. Shimada, J. Organomet. Chem. 2018, 869, 75-80.

[85] A. Glu, J. I. Schweizer, U. S. Karaca, W. Christian, M. Diefenbach, M. C. Holthausen, S. Schneider, Inorg. Chem. 2018, 57, 13822-13828.

[86] R. Corberán, M. Sanaú, E. Peris, J. Am. Chem. Soc. 2006, 128, 3974-3979.

[87] S. Sabater, J. A. Mata, E. Peris, ACS Catal. 2014, 4, 2038-2047. 
[88] J. F. Blez, A. Primo, A. M. Asiri, M. Álvaro, H. Garcí, Angew. Chemie - Int. Ed. 2014, 53, 12581-12586.

[89] A. Dhakshinamoorthy, P. Concepcion, H. Garcia, Chem. Commun. 2016, 52, 2725-2728.

[90] A. Dhakshinamoorthy, I. Esteve Adell, A. Primo, H. Garcia, ACS Sustain. Chem. Eng. 2017, 5, 2400-2406.

[91] T. Mitsudome, Y. Yamamoto, A. Noujima, T. Mizugaki, K. Jitsukawa, K. Kaneda, Chem. - A Eur. J. 2013, 19, 14398-14402.

[92] C. Wang, X. Lin, Y. Ge, Z. H. Shah, R. Lu, S. Zhang, RSC Adv. 2016, 6, 102102102108.

[93] D. Ventura-Espinosa, A. Carretero-Cerdán, M. Baya, H. García, J. A. Mata, Chem. - A Eur. J. 2017, 23, 10815-10821.

[94] D. Ventura-Espinosa, S. Sabater, A. Carretero-Cerdán, M. Baya, J. A. Mata, ACS Catal. 2018, 8, 2558-2566.

[95] P. Csabai, F. Joo, Organometallics 2004, 23, 5640-5643.

[96] J. Yang, P. S. White, C. K. Schauer, M. Brookhart, Angew. Chemie - Int. Ed. 2008, 47, 4141-4143.

[97] M. Bühl, F. T. Mauschick, Organometallics 2003, 22, 1422-1431.

[98] B. Bagh, D. W. Stephan, Dalton Trans. 2014, 43, 15638-15645. 



\section{CHAPTER 5}

\section{Gold Catalysis at the Surface of Graphene}

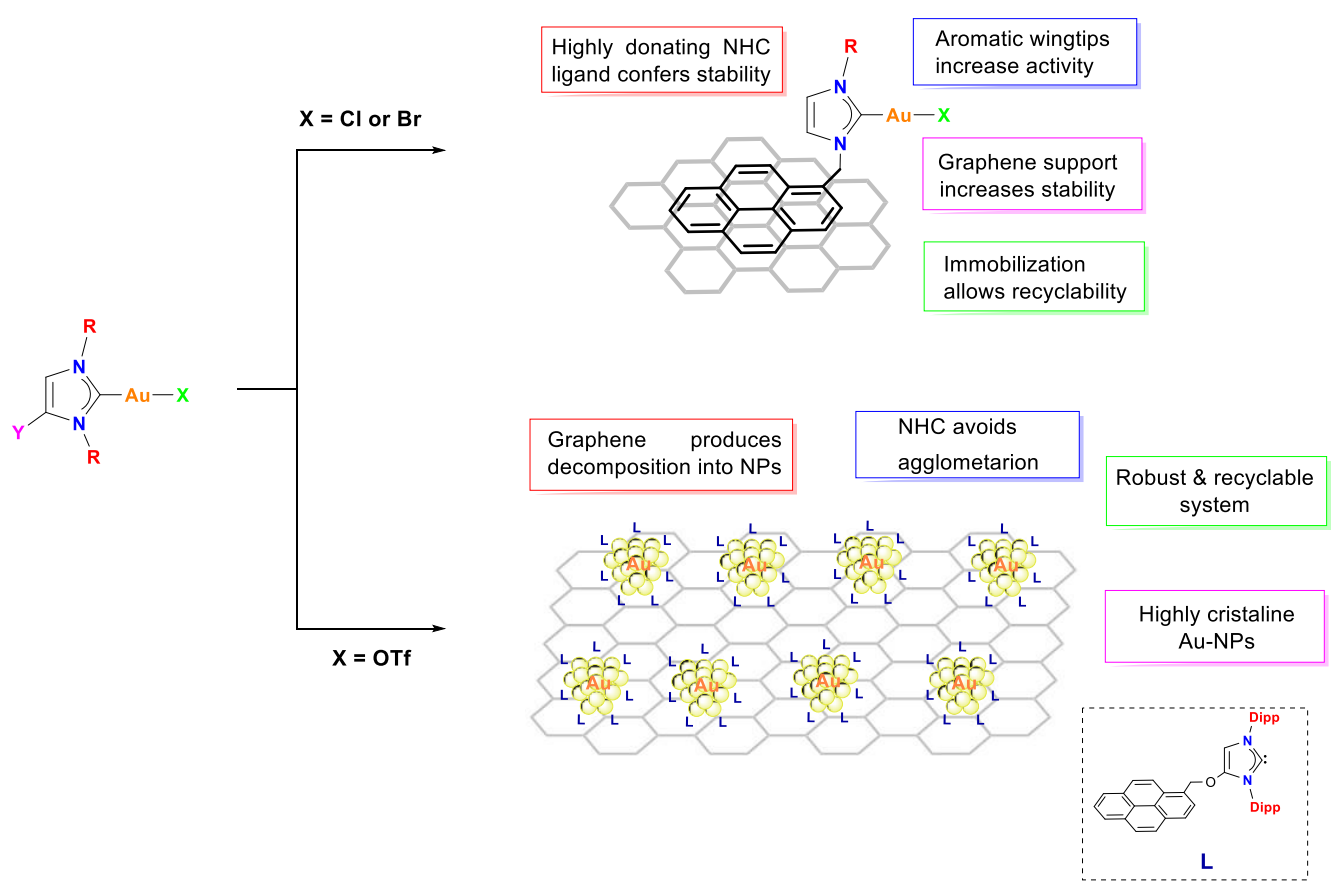





\subsection{INTRODUCTION}

This chapter is divided in two different parts. The first one deals with the catalytic applications of gold complexes immobilised onto $\mathrm{rGO}$. The second one describes a new methodology for the synthesis of gold nanoparticles functionalised with NHC ligands and grafted on graphene, and the study of the catalytic properties of the new material.

\subsubsection{Immobilisation of gold molecular complexes}

Although it was initially considered an inert metal, gold catalysis has emerged as a powerful tool to carry out numerous organic transformations. Gold shows catalytic activity in the form of metal complexes, nanoparticles and/or clusters. Each form has its advantages in terms of activity/selectivity and all of them have been widely studied. ${ }^{[1-3]}$ For the research in catalysis, the use of molecular organometallic complexes is especially interesting as it allows the study of the reaction mechanism, which provides information to design better catalysts. ${ }^{[4,5]}$ There are three common oxidation states for gold, $0,+1$ and +3 . Among them, gold (I) compounds are of particular interest because they are resistant to air and moisture and display high activity in different transformations such as nucleophilic reactions, ${ }^{[6,7]}$ cyclizations $^{[8,9]}$ and rearrangements, ${ }^{[10,11]}$ among others. ${ }^{[12]}$

Gold catalysts have been reported to assist in some selective reductions and oxidations. ${ }^{[13-16]}$ However, most of the catalytic reactions mediated by gold are based on the propensity of cationic gold (I) compounds to act as a Lewis acid towards the activation of $\pi$ bonds. ${ }^{[17]}$ This affinity allows the formation of new C-heteroatom bonds. In addition, the reactivity is not limited to C-C multiple bonds and other functional groups such carbonyls or imines can be reacted, giving rise to a plethora of organic transformations with a high atom economy.

From an economic point of view, gold is more abundant than other metals used in large-scale processes such as palladium, rhodium or platinum. In addition, the extraction of gold from mines every year is well-established and determines a high stability in its price. Nonetheless, gold is a precious metal with a high prize. ${ }^{[18]}$ That is why the successful implementation of gold in catalysis needs to fulfil two essential requirements:

i) The catalyst loadings have to be as low as possible

ii) The catalyst should be recyclable 
The use of low catalyst loadings is ligated to the development of highly active catalysts (i.e. high turnover number, TON). It is well-accepted that the vast majority of gold-catalysed reactions involve three stages. ${ }^{[5]}$

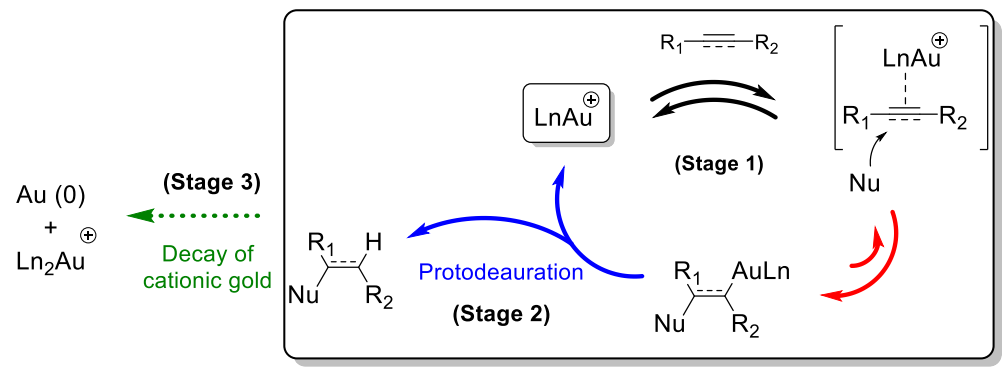

Figure 5.1 Typical steps in gold catalysed reactions. Adapted with permission from ref [5]. Copyright 2012 American Chemical Society

The first stage comprises the $\pi$-activation of an alkyne by cationic gold and subsequent nucleophilic attack on the [AuLn] ${ }^{+}$-activated alkyne, giving an alkenyl gold intermediate. In the second stage, the resulting vinyl complex reacts with an electrophile, most commonly a proton, to render the final product via protodeauration. In this step the gold catalytically active species is regenerated. The third stage is the deactivation of the catalyst and happens in a higher or lower extent depending on the reaction. In gold catalysis, deactivation is often ligated to the formation of gold mirror or inactive gold nanoparticles. Although there are several reactions that can be carried out with simple gold salts, ligand design plays a key role in the acceleration of reaction rates and stabilisation of active species, minimising catalyst deactivation.

Regarding the recyclability, different strategies have been proposed to recover gold complexes at the end of the catalytic reaction. The approach most commonly used is the formation of a covalent bond between a support and the molecular entity. Silica and mesoporous ordered silica materials are among the most frequently used supports to heterogenise gold molecular complexes. In these systems, silanol moieties act as grafting centres by means of a condensation reaction. Two approaches can be followed to anchor the molecular catalyst on silica. The first one involves the synthesis of a preformed gold complex that must contain a trialcoxysilyl group that condensates with the $\mathrm{Si}-\mathrm{OH}$ groups present at the surface of the silica (Figure $5.2, A) .{ }^{[19]}$ The second one implies the immobilisation of the organic ligand (which contains a trialcoxysilyl group) on the silica, prior to the complex formation (Figure 5.2, B). ${ }^{[20]}$ 

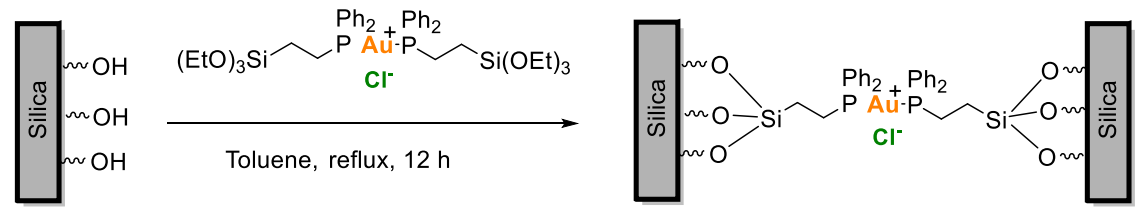

B $\frac{\frac{\delta}{\overline{\bar{c}}}}{\sim \mathrm{OH}}$
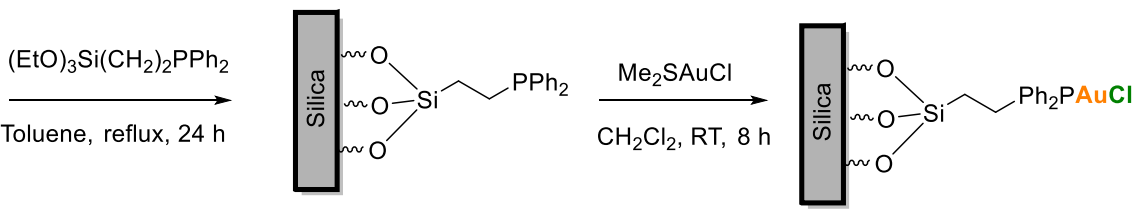

Figure 5.2 Direct synthesis (A) and stepwise synthesis (B) of gold complexes immobilised onto silica through a covalent bond

Using the first approach shown in Figure 5.2, Corma and coworkers reported in 2006 a silica-immobilised gold (I) catalyst that is active in the hydrogenation of alkenes. A higher reaction rate was observed for the grafted catalysts when compared with the homogeneous analogues, which the authors attributed to the site isolation effect (Figure 5.3). ${ }^{[21]}$ The good results obtained prompted them to the development of a new gold catalyst with an asymmetric ligand that is able to reduce alkenes to alkanes with excellent activities and good enantiomeric excess. The supported catalyst could be recycled up to 5 times without any decrease in activity, which gives an idea of the high stability of the material. ${ }^{[22]}$

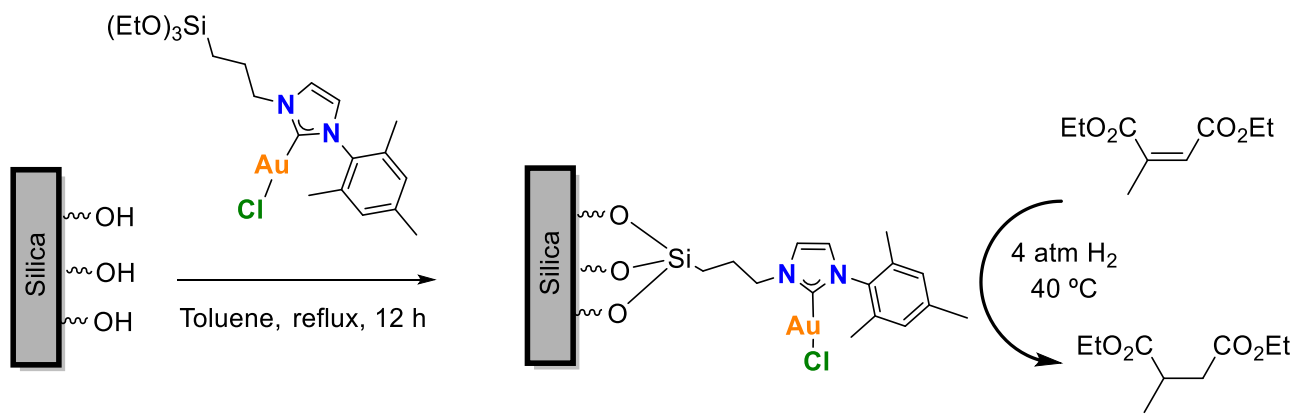

Figure 5.3 Heterogenised gold catalyst reported by Corma et al.

Years later, the group of Asensio studied the effect on catalysis of grafting an NHC-gold (I) complex through one or two centres (Figure 5.4). ${ }^{[23]}$ 


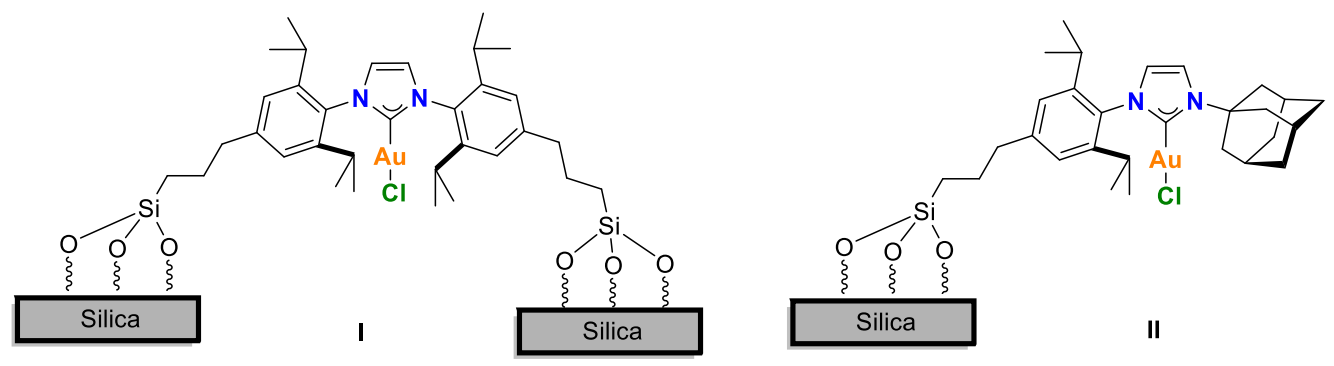

Figure 5.4 Gold (I) grafted catalyst on silica through two (I) or one (II) centres

The results concluded that immobilising the catalyst in one point was enough to develop a highly recyclable catalyst. No big differences could be observed when the catalyst anchored through two points (I in Figure 5.4) was used. Both catalysts were active and recyclable in typical homogeneous $A u(I)$ catalysed reactions such as hydration of alkynes, intramolecular hydroamination, intramolecular hydroarilation or cycloisomerization. Analysis of the reaction media after the recycling experiments showed that catalyst deactivation could not be attributed to metal leaching. Further studies demonstrated that the decay in catalytic activity was caused by a modification of the silica support during the magnetic stirred reactions. Moving to an orbital shaker allowed maintaining the morphology of the silica-based support. In fact, catalyst II could be used in a flow system (where no stirring is needed) affording excellent productivities and thus showing the high catalyst stability.

The immobilisation of gold is not restricted to silica and other materials such as magnetic nanoparticles, ${ }^{[24]}$ carbon nanotubes ${ }^{[25]}$ or modified polymers ${ }^{[26-29]}$ have been used. In particular, the use of polystyrene modified solids has found wide applications. However, the commercially available resins are commonly modified to generate suitable ligands on the surface, which adds extra steps to the synthetic protocol and modifies the properties of the support.

As an alternative to the formation of a covalent bond between the catalyst and the solid, non-covalent interactions can be the driving force to maintain the catalyst anchored to the solid support. This approach does not require prior functionalisation of the support and the weak interactions keep the structure of the support unaltered. On the contrary, if these interactions are too weak metal leaching could become an issue that leads to catalyst deactivation. Thus, catalyst design is crucial to maintain a good balance between activity and stability. 
Few reports have appeared in the literature for the immobilisation of gold (I) complexes using non-covalent interactions. In 2009 the immobilisation of a sulfonate tethered NHC-Au complex on a dendrimer through ionic interactions was reported. The dendrimer III could be isolated in good yield, but the catalytic properties of the system remained unstudied. ${ }^{[30]}$

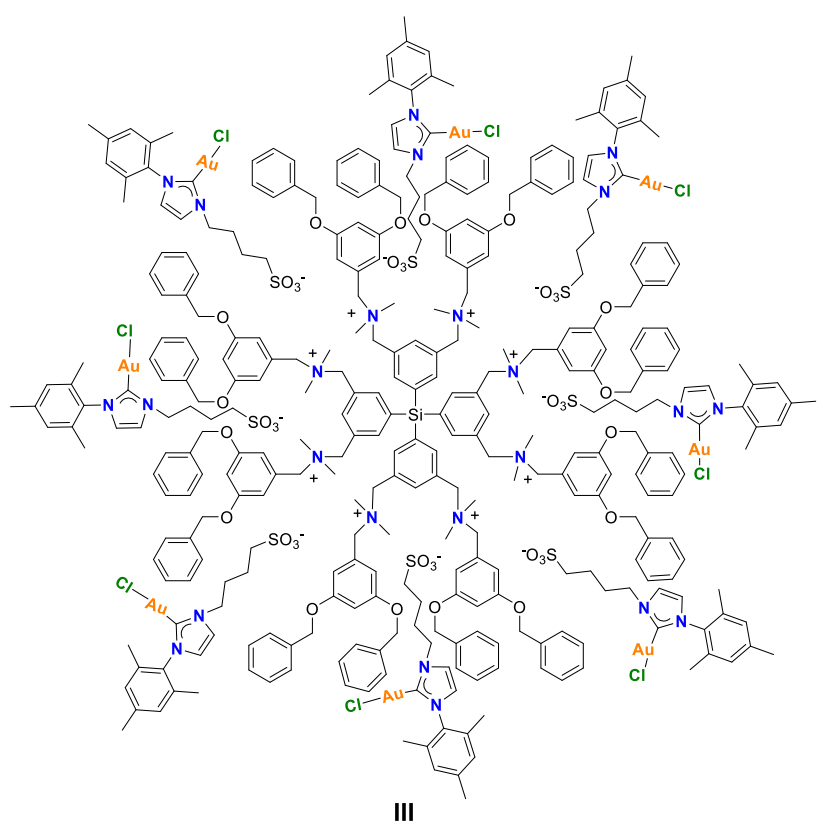

Figure 5.5 NHC-Gold(I) complex immobilised on a policationic dendrimer through ionic interactions

Recently, the use of carbonaceous materials as solid supports for catalysts has attracted a lot of attention. ${ }^{[31]}$ Carbon nanotubes and graphene-derived materials are excellent candidates owing to their thermal and chemical stability, mechanical resistance and high surface area. Due to the extended $\pi$-cloud of these materials, $\pi-\pi$ interactions are the non-covalent forces that keep the catalyst on the support.

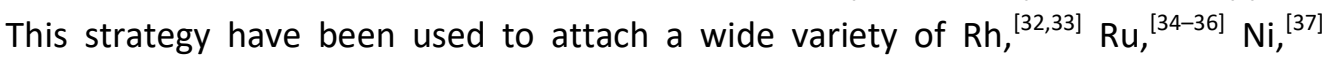
$\mathrm{Pd}^{[36,38,39]} \mathrm{Cu}^{[40]}$ or $\mathrm{Fe}^{[41]}$ compounds in carbon nanotubes or chemically derived graphenes. Despite the high development of the field, examples of the immobilisation of gold compounds through this methodology are scarce. In fact, the only example of a gold complex immobilised onto multi-walled carbon nanotubes through $\pi-\pi$ stacking interactions was reported in 2013 by S. Hermans and co-workers (Figure 5.6). ${ }^{[42]}$ 


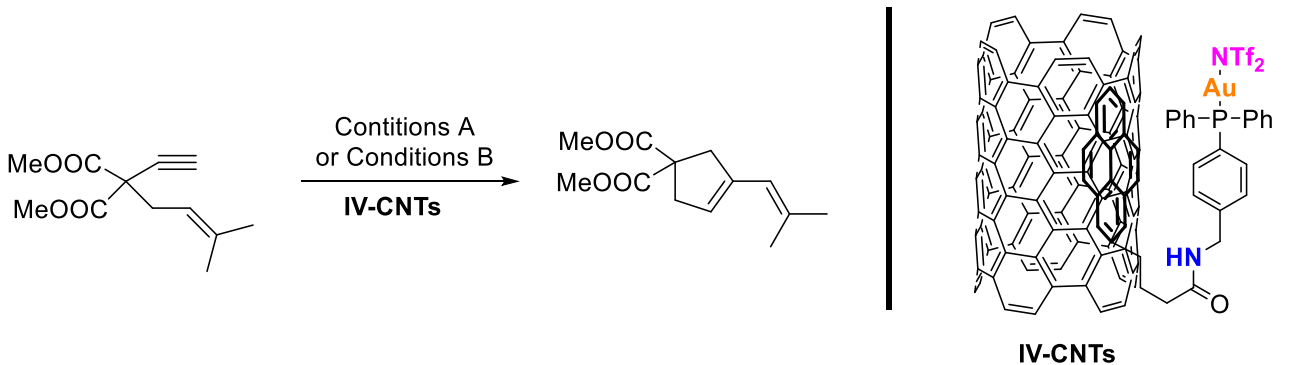

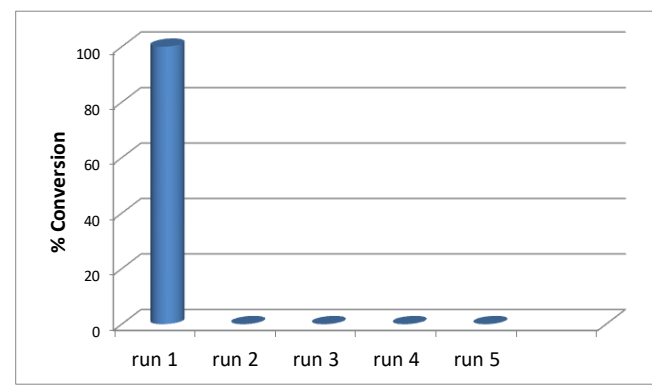

Conditions $\mathrm{A}: \mathrm{CH}_{2} \mathrm{Cl}_{2}$, RT, $30 \mathrm{~min}$

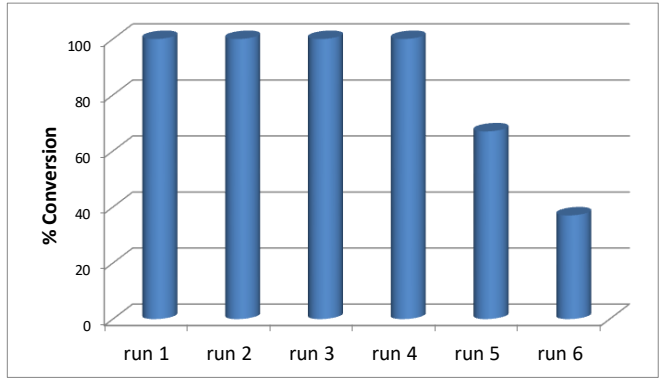

Conditions B: 1. $\mathrm{CH}_{2} \mathrm{Cl}_{2}, \mathrm{RT}, 30 \mathrm{~min}$

2. $-40^{\circ} \mathrm{C}, 20 \mathrm{~min}$

Figure 5.6 Gold complex anchored onto carbon nanotubes by $\pi-\pi$ stacking interactions and recycling properties

The authors reported a molecular gold complex that features a pyrene tag in its structure (IV). The presence of this polyaromatic group allowed the immobilisation of IV on CNTs using an easy and mild synthetic route. The soft conditions used preserved the molecular nature of IV without modification of the support. The hybrid material IV-CNTs was active in the cycloisomerisation of enynes and propargylic carbonates. The related homogeneous complex IV gave a similar activity, indicating that the support was not taking place in the catalytic process. The recyclability of catalyst IV-CNTs was studied. The results show that the temperature had a critical effect on the recyclability. The experiments were conducted in dichloromethane at room temperature. The catalyst was separated at the end of the reaction by centrifugation and used in the subsequent run. However, after the first cycle the activity of IV-CNTs completely decays and no conversion is observed in the following run. In a second set of experiments, the reaction mixture was cooled to $-40{ }^{\circ} \mathrm{C}$ prior centrifugation. Using this strategy the catalyst could be recycled up to four times without decrease in activity. After run 4 , the conversion decreased in runs 5 and 6 to 67 and $37 \%$ respectively. These results indicate that the catalysis was homogeneous in nature, with the boomerang effect taking place. During the 
reaction the complex is desorbed from the surface of CNTs and do the transformation. Then, the work-up for the separation of the catalyst from the reaction media is carried out at low temperatures, favouring the re-immobilisation of IV onto CNTs. Analysis by ICP-MS point to metal leaching as main cause of deactivation. Complex IV is gradually desorbed from CNTs and, after four runs, the metal content is too low to afford complete conversion.

\subsubsection{Generation and immobilisation of metal nanoparticles. Applications in catalysis}

Apart from gold molecular complexes, gold nanoparticles (Au-NPs) have emerged as

a potential tool in catalysis. The synthesis of Au-NPs is nowadays straightforward and the most common methods require the use of a reducing agent and a gold salt. Moreover, the tendency of gold (I) and gold (III) in many homogeneous systems is to precipitate with time and generate gold nanoparticles or clusters as highly active species. Additionally, if the Au NPs are captured in the presence of a support, a potential recyclable catalyst is obtained.

Supported gold nanoparticles are usually prepared by deposition-precipitation of gold salts or by adsorption of preformed gold nanoparticles. ${ }^{[1]}$ The depositionprecipitation method is the most widely used to obtain ligandless gold nanoparticles on metal oxides (Figure 5.7). It consists in the generation of $\mathrm{Au}(\mathrm{OH})_{3}$ from ions (usually $\mathrm{AuCl}_{4}{ }^{-}$) by a raise in the $\mathrm{pH}$. As the $\mathrm{pH}$ increases the chloride anions are replaced by hydroxyl groups, generating $\mathrm{Au}(\mathrm{OH})_{3}$ which is rapidly adsorbed on the surface of metal oxides. The next step includes nucleation and growth of the nanoparticle. Here, the aurophilicity of gold (i.e. the tendency to form Au-Au bonds) plays a key role. Once the gold atoms are organized in small nuclei its growth will determine the final size of the nanoparticle. The last stage of this protocol implies the reduction of the gold ions to form the metal clusters. This reduction is usually carried out by thermal treatment of the solid under a hydrogen flow. 
One of the most important roles of the support is to avoid agglomeration, which would cause excessive growth and generate inactive gold nanoparticles. ${ }^{[1]}$ The presence of defects and the loading of gold are the factors that also determine the final size of gold nanoparticles.

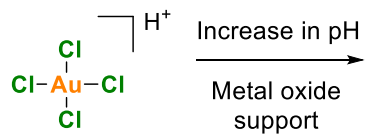

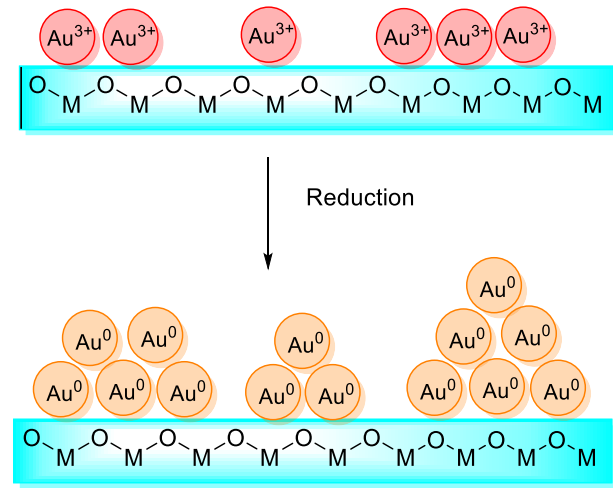

Figure 5.7 Schematic representation of the deposition-precipitation method

The deposition-precipitation method does not work when carbonaceous materials are used as supports. To circumvent this problem, an alternative route has been designed to immobilize Au-NPs on carbon-derived materials. The methodology consists in the pre-formation of a colloidal dispersion of gold nanoparticles that are then adsorbed on the support. The preparation of colloidal gold nanoparticles is a well-established procedure. Starting from a diluted aqueous solution of tetrachloroauric acid a fairly homogeneous size distribution of Au-NPs can be obtained by reduction with citric acid. The freshly prepared gold nanoparticles undergo rapid agglomeration if they are not stabilised by ligands (vide infra) or supported on a solid matrix (Figure 5.8).

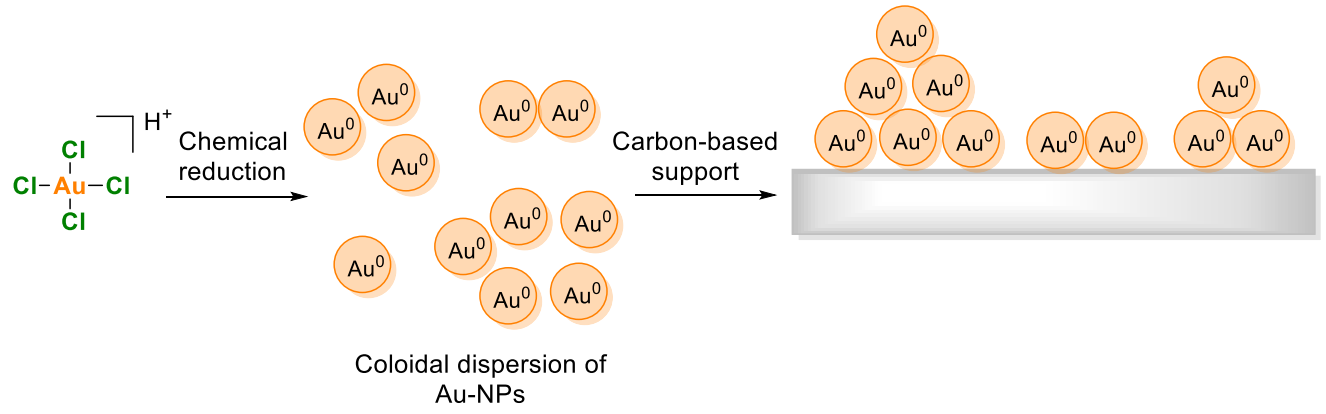

Figure 5.8 Capture of pre-formed gold nanoparticles by a carbonaceous solid matrix 
Colloidal gold nanoparticles can be stabilised with ligands to avoid the agglomeration. Thiols are among the first organic molecules used for the functionalisation of gold surfaces. ${ }^{[4]}$ The strong Au-S bond together with the high packing density of long-chain alkyl thiols on the surface of gold affords Au-NPs with a narrow size distribution. However, this high packing may prevent the access of the reactants to the surface of gold, rendering an inactive catalyst. ${ }^{[44]}$ Therefore a careful ligand design is needed to find a good balance between stabilisation and activity of Au-NPs (Figure 5.9). ${ }^{[45,46]}$

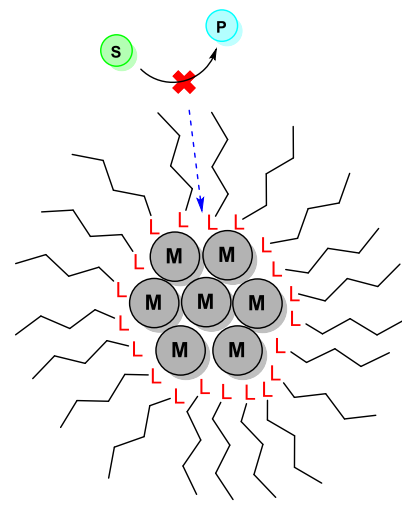

- Ligand allows high packing

- Difficult access of substrates to the active sites

- Low activity

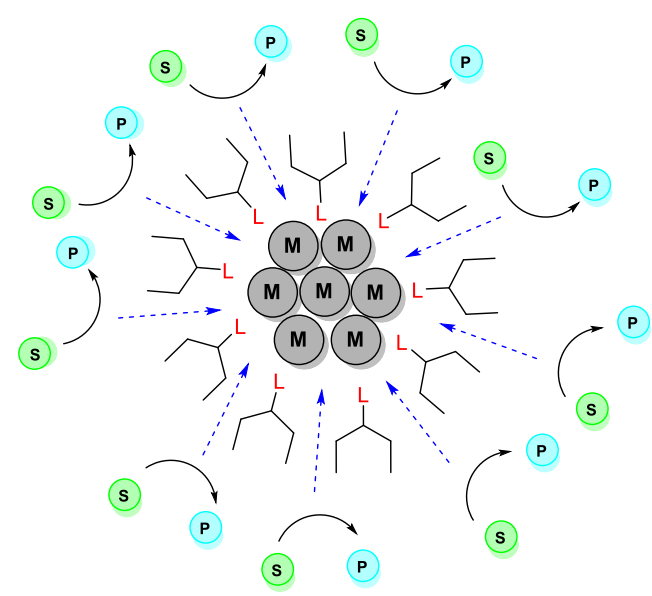

- Sterically demanding ligand - Ligand stabilised nanoparticles with available channels to the active sites - High activity

Figure 5.9 Different scenarios in ligand-stabilised nanoparticles

Apart from thiols, the functionalisation of gold nanoparticles have been extended to other organic molecules such as carboxylic acids, ${ }^{[47]}$ amines, ${ }^{[48]}$ alkynes, ${ }^{[49]}$ phosphines, $^{[50]}$ secondary phosphine oxides $^{[51]}$ or carbenes. ${ }^{[52,53]}$ Among them, $\mathrm{N}$-heterocyclic carbenes $(\mathrm{NHC})$ have attracted a lot of interest for the functionalisation of gold surfaces. As a result of the extensive study in organometallic chemistry and catalysis, a wide range of NHCs structures are reported. The high synthetic versatility of NHCs allows the fine tuning of the electronic and steric properties of the ligand. Besides, ligand precursors (typically imidazolium salts) are stable and easily accessible. NHCs are known for his strong $\sigma$-donating character, which provides strong metal-NHC bonds and ensures that the ligand will not be released from the surface of the nanoparticle. ${ }^{[53]}$ That is why gold 
nanoparticles capped with NHCs show improved stability compared to other ligands such as phosphines or thiolates.

NHC-stabilised gold nanoparticles were reported by T. D. Tilley for first time in 2009. ${ }^{[54]}$ Starting from a well-defined $\mathrm{Au}(\mathrm{NHC}) \mathrm{Cl}$ compound the reduction using $\mathrm{KBEt}_{3} \mathrm{H}$ or 9-BBN (9-borabicyclo [3.3.1] nonane) afforded monodisperse NHC-functionalised gold nanoparticles with an average diameter of 5-7 nm (Figure 5.10). Regarding the morphology, the nanoparticles were mostly spherical, although some triangularly-shaped NPs were also present. These nanoparticles were soluble in most non-chlorinated organic solvents and stable for long periods.
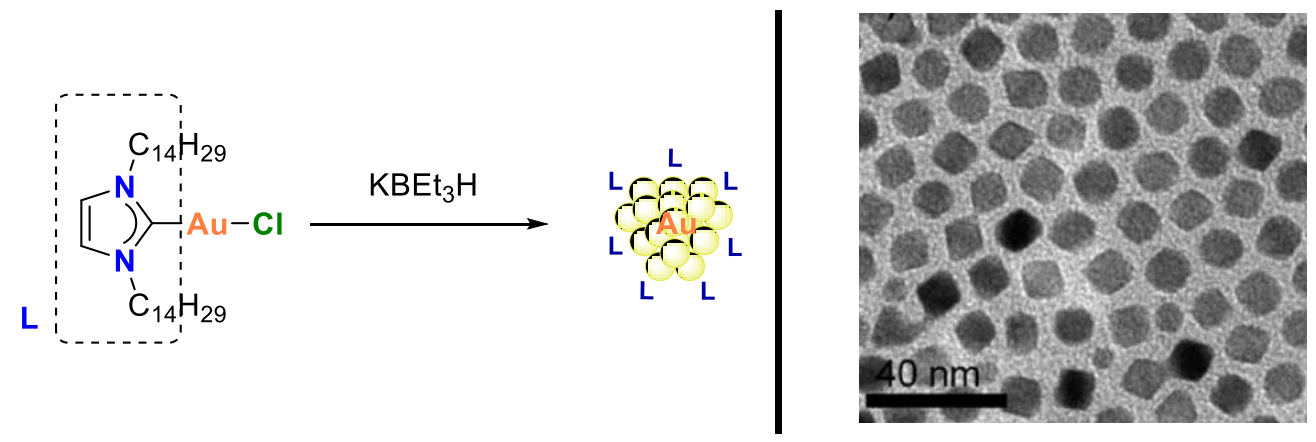

Figure 5.10 NHC-stabilised gold nanoparticles reported by Tilley. Reproduced from ref.[54] with permission from The Royal Society of Chemistry

Despite the high development in the field of Au-NPs capped with NHC ligands, ${ }^{[53]}$ applications in catalysis remain scarce. ${ }^{[55-58]}$ Prof. D. Toste and co-workers prepared Au-NPs functionalised with different NHCs ligands by reduction of the corresponding $\mathrm{Au}(\mathrm{NHC}) \mathrm{Cl}$ complexes using $t \mathrm{BuNH}_{2} \mathrm{BH}_{3}$ or $\mathrm{NaBH}_{4}$ as reductants. The nanoparticles formed were embedded in a dendrimer to further prevent aggregation, and immobilised on silica to facilitate catalyst recycling (Figure 5.11 ). ${ }^{[58]}$ The positive effect of the ligand caused a dramatic increase in the activity of the catalyst for the intramolecular lactonization of allenes. While the NHC-stabilised gold nanoparticles were active at $20{ }^{\circ} \mathrm{C}(100 \%$ yield, $22 \mathrm{~h})$, ligandless gold nanoparticles were completely inactive below $80^{\circ} \mathrm{C}$, and an increase in the temperature up to $100{ }^{\circ} \mathrm{C}$ was needed to afford complete conversion in 22 hours. In addition, the active gold nanoparticles could be recycled five times without any decrease in activity. This report by Toste constitutes one of the few examples that merge the use of ligand-stabilised gold nanoparticles and supports. 


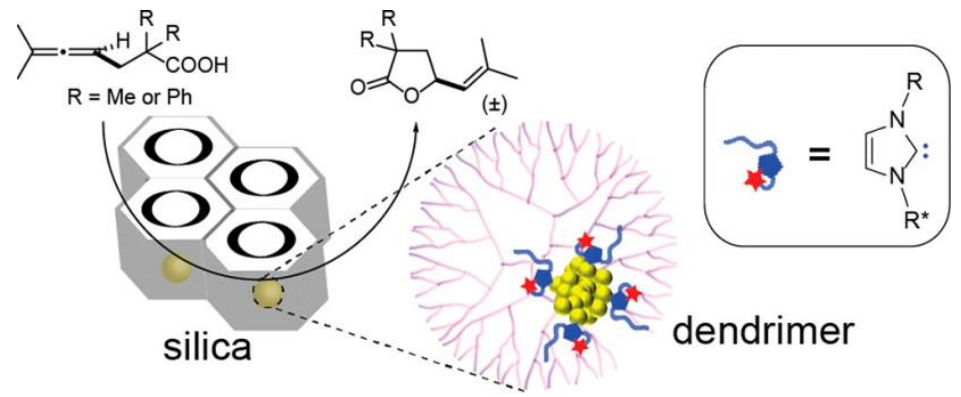

Figure 5.11 NHC-stabilised Au-NPs encapsulated in a dendrimer and loaded into Silica reported by Toste et al. Reprinted with permission from ref [58]. Copyright 2018 American Chemical Society 


\subsection{RESULTS AND DISCUSSION}

\subsubsection{Molecular complexes anchored onto reduced graphene oxide}

\subsubsection{Synthesis and characterisation of ligand precursor $H$ and gold complex $10 \mathrm{H}$}

To study the catalytic properties of gold complexes grafted onto rGO, two metal compounds bearing an NHC ligand with a pyrene tag were prepared. The ligand precursors used are depicted in Figure 5.12.

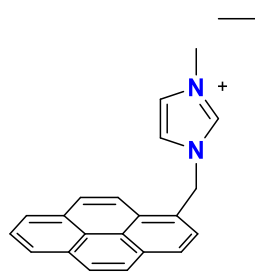

C

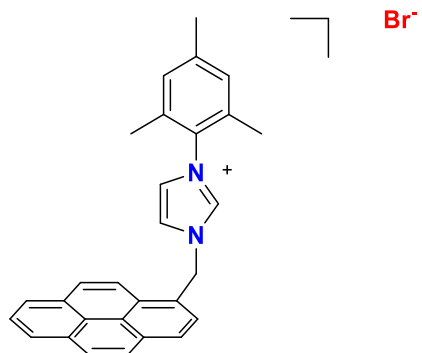

H

Figure 5.12 Ligand precursors discussed in this section

Along with the previously discussed imidazolium salt $\mathbf{C}$, imidazolium salt $\mathbf{H}$ was synthesized in one step. Alkylation of 1-mesityl imidazole with 1-(bromomethyl)pyrene in refluxing THF for $16 \mathrm{~h}$ afforded $\mathbf{H}$ as an analytically pure white solid in 90 \% yield. Compound $\mathbf{H}$ was characterised by NMR spectroscopy, ESI-MS and elemental analysis.

Different metalation strategies were followed to obtain NHC-coordinated gold complexes (Figure 5.13). Complex 9C was synthesised by transmetallation of the preformed silver-NHC complex, which was obtained by treatment of $\mathbf{C}$ with $\mathrm{Ag}_{2} \mathrm{O}$ in refluxing $\mathrm{MeCN}$ under the exclusion of light. After 5 hours, the metal precursor [AuCl(SMe $\left.\mathrm{SM}_{2}\right]$ was added along with an excess of $\mathrm{KCl}$ and stirred overnight at room temperature. Complex 9C was isolated in $81 \%$ yield as a white solid after crystallization from a dichloromethane/hexane mixture. 


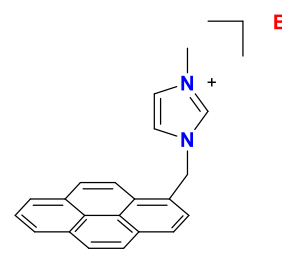

C

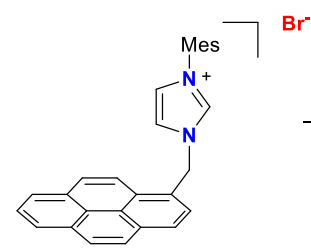

H

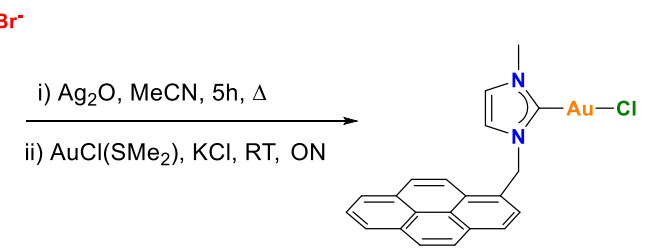

$9 \mathrm{C}$

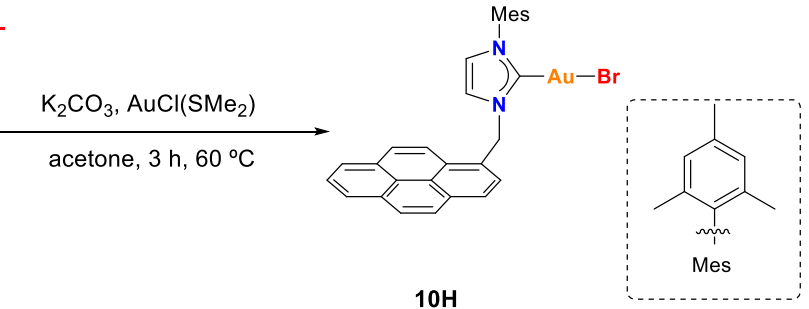

Figure 5.13 Synthesis of gold complexes $9 \mathrm{C}$ and $10 \mathrm{H}$

Complex $\mathbf{1 0 H}$ was obtained by deprotonation of imidazolium salt $\mathbf{H}$ using potassium carbonate. Following the procedure reported by Nolan, imidazolium salt $\mathbf{H}$, $\mathrm{AuCl}\left(\mathrm{SMe}_{2}\right)$ and potassium carbonate were suspended in $2 \mathrm{~mL}$ of acetone in a vial and the mixture was stirred at $60^{\circ} \mathrm{C}$ for 3 hours. ${ }^{[59]}$ Complex $10 \mathrm{H}$ was obtained in a $66 \%$ yield after purification by column chromatography and recrystallization from dichloromethane/pentane. Both complexes were completely characterised by NMR, ESI-MS, elemental analysis and X-Ray diffraction (see chapter 6).

\subsubsection{Immobilisation of $9 \mathrm{C}$ and $10 \mathrm{H}$ onto $\mathrm{rGO}$}

Complexes $\mathbf{9 C}$ and $\mathbf{1 0 H}$ were anchored onto rGO using a methodology previously reported by us (Figure 5.14). ${ }^{[36,60]}$

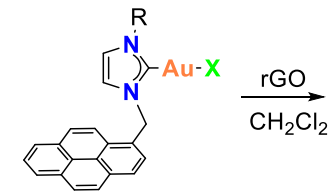

9C, $\mathrm{R}=\mathrm{Me}, \mathrm{X}=\mathrm{Cl}$ 10H, $\mathrm{R}=$ Mes, $\mathrm{X}=\mathrm{Br}$

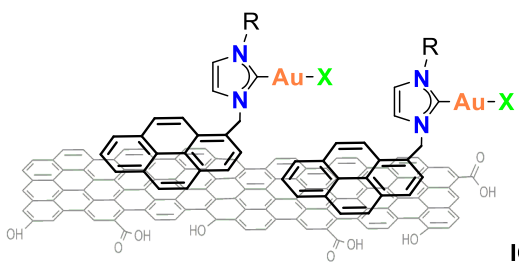

9C-rGo, $\mathrm{R}=\mathrm{Me}, \mathrm{X}=\mathrm{Cl} \quad 3.9 \% \mathrm{wt}$ 10H-rGO, R = Mes, $X=B r \quad 0.9 \% w t$

Figure 5.14 Immobilisation of $9 \mathrm{C}$ and $10 \mathrm{H}$ on $\mathrm{rGO}$ 
The two hybrid materials were characterised by TEM and XPS, and the exact gold content in each solid was determined by ICP-MS after digestion of the samples in nitric acid (Figure 5.14).

HRTEM images of $\mathbf{9 C}$-rGO and $\mathbf{1 0 H}-\mathbf{r G O}$ show the characteristic morphology of reduced graphene oxide which has not been altered after the immobilisation process. No metal nanoparticles can be observed, and the EDS spectrum show the presence of gold on the surface of graphene, which indicate the molecular nature of the species grafted on rGO.

Further evidence of the presence of the molecular compound $10 \mathrm{H}$ on $\mathrm{rGO}$ was obtained by XPS analysis (Figure 5.15). Comparison of the spectra of the molecular complex $10 \mathrm{H}$ and the hybrid material $10 \mathrm{H}-$-rGO show the characteristic core-level peaks of Au4f, Br3d and N1s at the same binding energy, showing that $10 \mathrm{H}$ is on the surface of rGO. XPS analysis shows that the soft conditions used during the synthesis of $\mathbf{1 0 H}-\mathrm{rGO}$ prevent changes in the properties of both, the gold complex and the support.
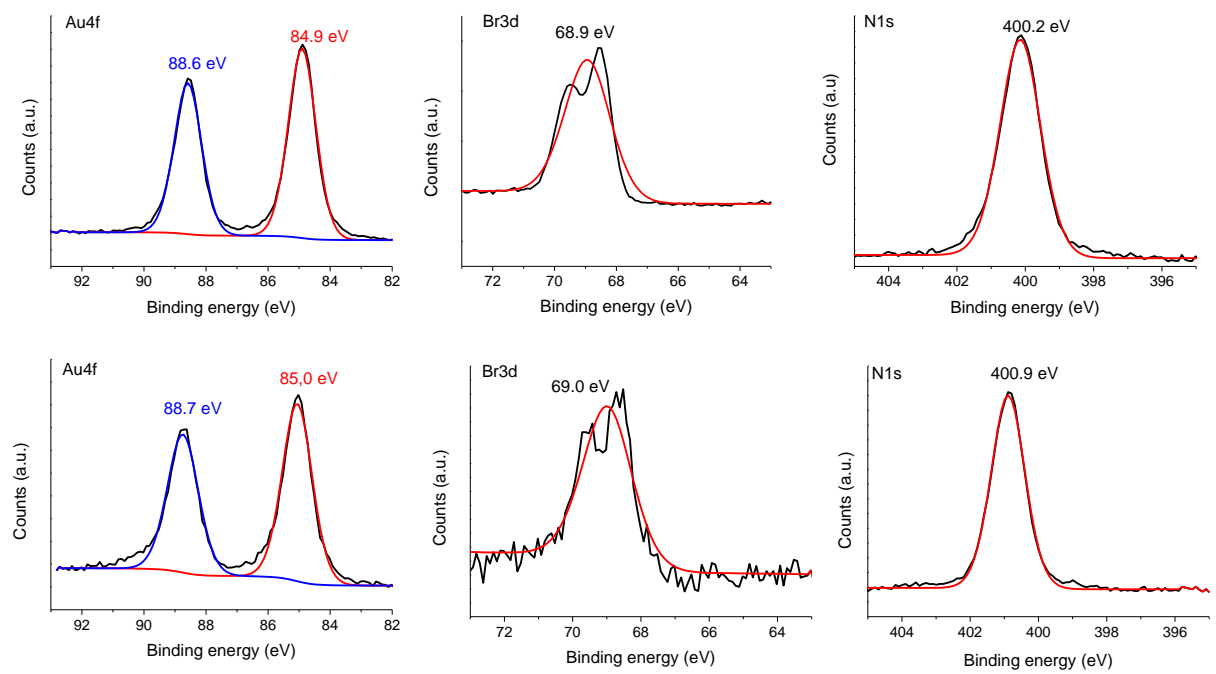

Figure 5.15 Comparative XPS analysis of $\mathbf{1 0 H}$ (top) and 10H-rGO (bottom)

\subsubsection{Catalytic properties of gold molecular complexes and the related hybrid materials}

The catalytic activity of complexes $\mathbf{9 C}$ and $\mathbf{1 0 H}$ and the related hybrid materials 9C-rGO and $\mathbf{1 0 H}-$ rGO was tested in the intramolecular hydroamination of alkynes. In order to generate the cationic gold (I) active species, a halide abstractor such as 
silver tetrafluoroborate $\left(\mathrm{AgBF}_{4}\right)$ was added. ${ }^{[61]}$ For the optimisation of the conditions, the reactions were initially performed at $50{ }^{\circ} \mathrm{C}$, under air and using technical solvents.

Table 5.1 Optimisation of reaction conditions

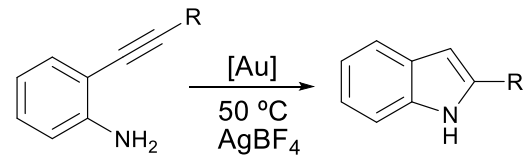

\begin{tabular}{|c|c|c|c|c|c|c|}
\hline Entry & Catalyst & {$[\mathrm{Au}](\mathrm{mol} \%)$} & $\mathbf{R}$ & Solvent & Time (h) & $\begin{array}{c}\text { Conversion } \\
(\%)^{\mathrm{a}}\end{array}$ \\
\hline 1 & - & - & $\mathrm{Ph}$ & Toluene & 6 & 0 \\
\hline $2^{b}$ & - & - & $\mathrm{Ph}$ & $"$ & 6 & 0 \\
\hline 3 & rGO & - & $\mathrm{Ph}$ & $"$ & 6 & 0 \\
\hline 4 & 9C & 1 & $\mathrm{Ph}$ & $"$ & 6 & 20 \\
\hline 5 & $10 \mathrm{H}$ & 1 & $\mathrm{Ph}$ & $"$ & 6 & 91 \\
\hline 6 & $10 \mathrm{H}$ & 1 & $\mathrm{Ph}$ & $"$ & 24 & 100 \\
\hline 7 & $9 \mathrm{C}$ & 1 & $\mathrm{nBu}$ & $"$ & 8 & 32 \\
\hline 8 & $9 \mathrm{C}$ & 1 & $\mathrm{nBu}$ & $"$ & 24 & 40 \\
\hline 9 & $10 \mathrm{H}$ & 1 & $\mathrm{nBu}$ & $"$ & 8 & 50 \\
\hline 10 & $10 \mathrm{H}$ & 1 & $\mathrm{nBu}$ & $"$ & 24 & $71(65)$ \\
\hline $11^{c}$ & $10 \mathrm{H}$ & 1 & $\mathrm{Ph}$ & $"$ & 6 & 71 \\
\hline 12 & $10 \mathrm{H}$ & 2 & $\mathrm{Ph}$ & $"$ & 1 & $100(90)$ \\
\hline $13^{d}$ & $10 \mathrm{H}$ & 2 & $\mathrm{Ph}$ & $"$ & 1 & 100 \\
\hline 14 & $10 \mathrm{H}$ & 2 & $\mathrm{Ph}$ & THF & 6 & 15 \\
\hline 15 & $10 \mathrm{H}$ & 2 & $\mathrm{Ph}$ & $\mathrm{MeCN}$ & 1 & 100 \\
\hline 16 & 9C-rGO & 1 & $\mathrm{Ph}$ & Toluene & 6 & 82 \\
\hline 17 & $10 \mathrm{H}-\mathrm{rGO}$ & 0.08 & $\mathrm{Ph}$ & Toluene & 1.5 & 100 \\
\hline 18 & $10 \mathrm{H}-\mathrm{rGO}$ & 0.08 & $\mathrm{Ph}$ & $\mathrm{MeCN}$ & 24 & 93 \\
\hline 19 & $10 \mathrm{H}-\mathrm{rGO}$ & 0.56 & $\mathrm{Ph}$ & Toluene & 0.2 & 100 \\
\hline
\end{tabular}

Reaction conditions: Reactions carried out at $50{ }^{\circ} \mathrm{C}$ and at a concentration of $0.0625 \mathrm{M}$ using 0.2 or $0.5 \mathrm{mmol}$ of substrate and 3 eq. of $\mathrm{AgBF}_{4}$ respect to gold catalyst. [a] Conversion determined by $\mathrm{GC}$ analysis using anisole as the internal standard. Isolated yields in parenthesis. [b] Reaction carried out in the presence of $6 \mathrm{~mol} \%$ of $\mathrm{AgBF}_{4}$. [c] Reactions carried out at a substrate concentration of $0.03125 \mathrm{M}$. [d] Reaction carried out under $\mathrm{N}_{2}$ atmosphere.

Table 5.1 (entry 1) shows that the reaction does not work in the absence of a catalyst. Blank experiments performed using silver tetrafluoroborate and rGO demonstrate that neither the halide scavenger nor the graphenic material are active in this transformation (entries 2 and 3). Gold complexes $\mathbf{9 C}$ and $\mathbf{1 0 H}$ are active catalysts for this reaction, albeit with different activity. For instance, complex $9 \mathrm{C}$ 


\section{Chapter 5}

showed a low $20 \%$ conversion after $6 \mathrm{~h}$ at a catalyst loading of $1 \mathrm{~mol} \%$ (entry 4). However, under the same reaction conditions complex $\mathbf{1 0 H}$ afforded a $91 \%$ conversion (entry 5). This tendency was double-checked with a different substrate by analysing the catalytic results of the reactions at different times using catalysts $9 \mathrm{C}$ and $\mathbf{1 0 H}$ (entries 7-10). Further experiments revealed that with catalyst $10 \mathrm{H}$ the reaction was complete in $1 \mathrm{~h}$ when the catalyst loading was increased up to $2 \mathrm{~mol} \%$ (entry 12). No differences in reactivity were observed when the reaction was performed under a nitrogen atmosphere (entry 13). The solvent effect was also studied (entries 12, 14, 15). The reaction worked well in toluene and in acetonitrile, but a marginal $15 \%$ yield was obtained in THF.

Interesting results were observed when moving to the hybrid materials 9 C-rGo and 10H-rGO. While the molecular complex 9C afforded a $20 \%$ conversion after 6 hours, the heterogeneous version 9C-rGO accounted for a $81 \%$ conversion under the same reaction conditions (compare entries 4 and 16). This effect was even more evident in the case of the catalyst featuring a mesityl group. In this situation, the molecular complex $\mathbf{1 0 H}$ afforded a $91 \%$ conversion after 6 hours at a loading of $1 \mathrm{~mol} \%$. On the contrary, when using $\mathbf{1 0 H}-\mathbf{r G O}$ the catalyst loading could be decreased up to $0.08 \mathrm{~mol} \%$ to afford a $100 \%$ conversion in $90 \mathrm{~min}$. The low catalyst loading used with $10 \mathrm{H}-\mathrm{rGO}$ is noteworthy, especially considering that gold-mediated intramolecular hydroaminations usually require $1-2$ mol \% of catalyst loading. ${ }^{[62,63]}$

To obtain a comparative analysis of the influence of the ligand and the support on the catalysis, the performance of the two metal complexes and the two hybrid materials was monitored by GC analysis (Figure 5.16). 


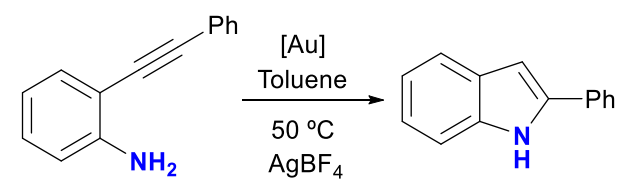

a)

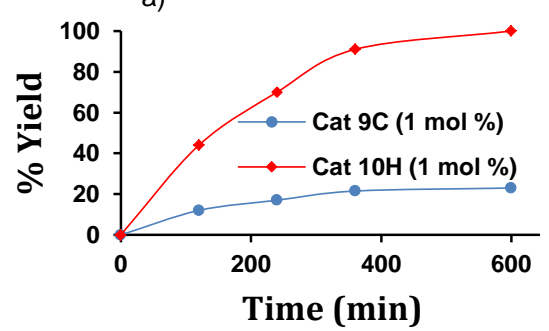

c)

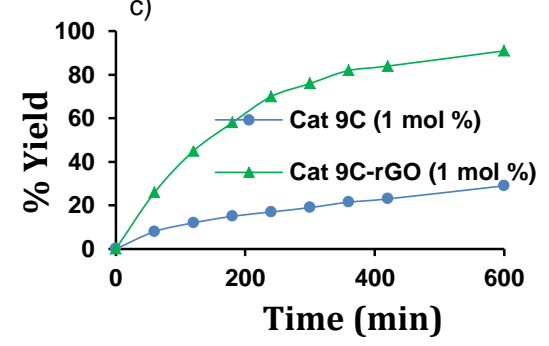

b)

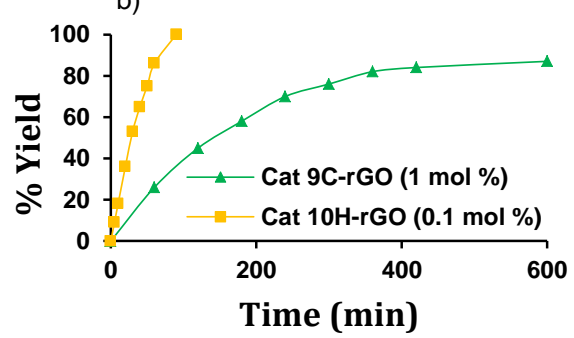

d)

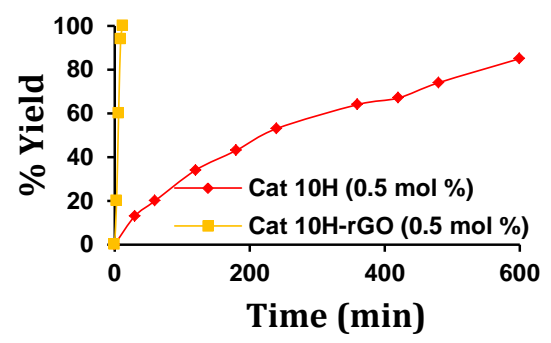

Figure 5.16 Reaction monitoring of the intramolecular hydroamination reaction using different catalysts

First, the effect of the ligand was checked (Figure 5.16, a and b). The catalytic activity of complexes $\mathbf{9 C}$ and $\mathbf{1 0 H}$ is remarkably different. Both complexes have the same general structure. The only difference is the substituent at one of the nitrogen atoms of the NHC. The molecular catalyst with a methyl group (9C) is not very active in this reaction and affords only a $20 \%$ conversion after 10 hours. Substitution of the methyl group for a mesityl group completely changes the activity and a $100 \%$ conversion is obtained under the same reaction conditions. This situation is also observed when using the hybrid materials. The supported catalyst $\mathbf{9 C}-\mathbf{r G O}$ reaches $85 \%$ conversion after 400 minutes. Extended reaction times did not improve the result, indicating catalyst deactivation. On the contrary, with catalyst $\mathbf{1 0 H}-\mathbf{r G O}$ the reaction is complete after 90 min using tenfold less catalyst loading $(0.1 \mathrm{~mol} \%)$. These results show that ligand design plays a key role in determining the stability of the catalyst. The introduction of aromatic substituents at the nitrogen position of the NHC increases stability and activity.

Even more interesting is the effect of the support (Figure 5.16, c and d). With catalyst $9 \mathrm{C}$ the reaction rates are slow and the reaction is gradually stopped when a 
$20 \%$ conversion is reached, indicating limited stability of the molecular complex. However, a different behaviour is observed when the complex is grafted on rGO. In this case, the activity of the molecular compound is enhanced and an $85 \%$ conversion is obtained under the same reaction conditions. Immobilisation of complex 9C on rGO has a beneficial effect on catalysis. This effect is even more drastic in the case of $\mathbf{1 0 H}$ and $\mathbf{1 0 H}-\mathbf{r G O}$. The molecular complex $\mathbf{1 0 H}$ affords an $80 \%$ conversion after 10 hours at a catalyst loading of 0.5 mol \%, but when $10 \mathrm{H}-\mathrm{rGO}$ is used the rate of the reaction is extremely enhanced and complete conversion is obtained in just 12 minutes. The reaction profile for $\mathbf{1 0 H}$ and $\mathbf{1 0 H}-\mathrm{rGO}$ shows a big difference in reaction rates. The fact of immobilizing a molecular catalyst on a solid material usually has a detrimental effect on kinetics due to diffusion problems of the reactants that find more difficulties in finding the active centres. This is not the case for $\mathbf{1 0 H - r G O}$ because graphene is a flat 2D material that do not hind the metal centres. In addition the reaction rates are not affected but increased, most probably due to limit degradation of the active catalyst. Although we do not have a suitable explanation for the influence of graphene in catalysis, this effect is general and has been observed in other catalytic reactions using graphene doped with other metals. $^{[64-66]}$

To gain more insight into the nature of active species, the hot filtration test was performed. Using the optimized conditions (Table 5.1, entry 17) the reaction was set up. After 45 minutes (conversion $53 \%$ ) the hybrid material 10H-rGO was separated by filtration at the reaction temperature $\left(50{ }^{\circ} \mathrm{C}\right)$. The filtrate was left to react at $50{ }^{\circ} \mathrm{C}$ for 5 hours. After this time, GC analysis reveals that no more product had been formed. In a parallel experiment, the separated catalyst was treated with more substrate and the reaction heated for 2 hours, showing complete conversion of the starting material. The results show that $10 \mathrm{H}$ remains anchored on $\mathrm{rGO}$ under the working conditions and discards any homogeneous species taking part during the catalysis.

Next, the stability of catalyst $\mathbf{1 0 H}-$ rGO towards recyclability was studied. The experiments were conducted under the conditions shown in Table 5.1 (entry 19). After each run, the catalyst was separated by decantation, washed with toluene and used in the following run. Silver tetrafluoroborate was only added in the first run, indicating that the active species is formed at the beginning of the reaction and then is stabilised on the rGO support. The reaction monitoring profile (Figure 5.17) shows that $\mathbf{1 0 H}-\mathbf{r G O}$ can be reused for three times without any decrease in activity. After the third run some deactivation is taking place, as the activity slightly decrease in run 
four. This tendency becomes more evident in runs five and six, where extended reaction times are required to accomplish full conversion. Nonetheless, even after five runs the activity of $\mathbf{1 0 H}-\mathrm{rGO}$ is superior to that of $\mathbf{1 0 H}$ (see Figure $5.16, \mathrm{~d}$ ).
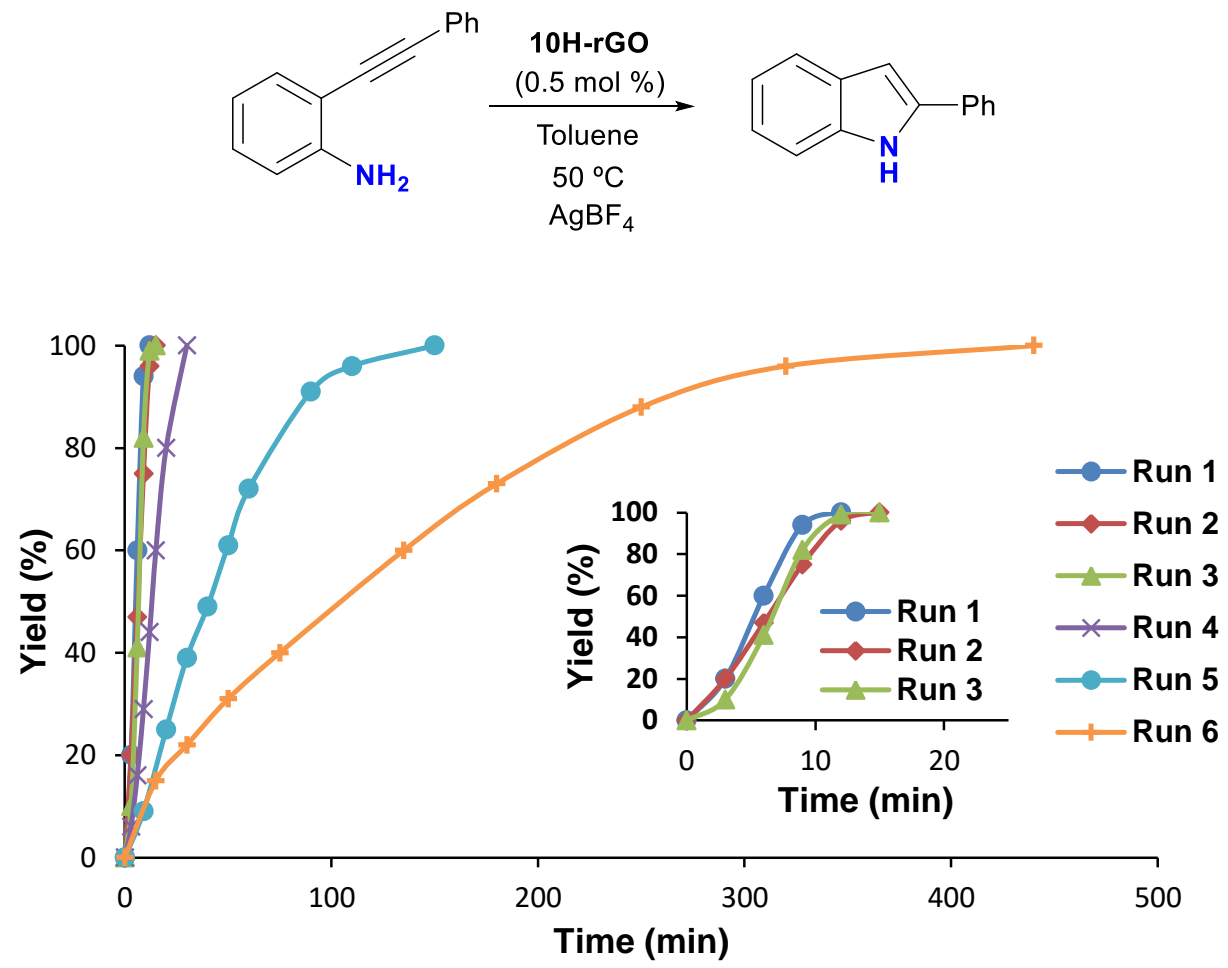

Figure 5.17 Recycling experiments in the intramolecular hydroamination reaction using $10 \mathrm{H}-\mathrm{rGO}$ as catalyst

In order to study the factors that govern the deactivation of the catalyst, the gold content in the reaction media was analysed after each run. The results show that the gold content in runs 1-3 is negligible, which correlates with the good performance of the catalyst during the first three runs, with scarce loose of activity. On the contrary, the gold content in the solid after run 6 is $c a .50 \%$ of the initial gold loading. These results indicate that leaching is one of the reasons that cause catalyst deactivation, although other mechanisms such as complex degradation may be operating.

The morphology of the hybrid material after six catalytic runs was analysed by HRTEM. Comparison of the images before and after the recycling experiment show that after the catalysis the graphene preserves its 2D nature (Figure 5.18). The morphology of the graphene sheets remains unaltered. The only difference is the appearance of more wrinkles, which is attributed to the mechanical stirring during 
the catalytic reactions. No gold nanoparticles were observed, and EDS analysis reveals the presence of gold on the surface of $\mathrm{rGO}$, indicating that the active species should be molecular.

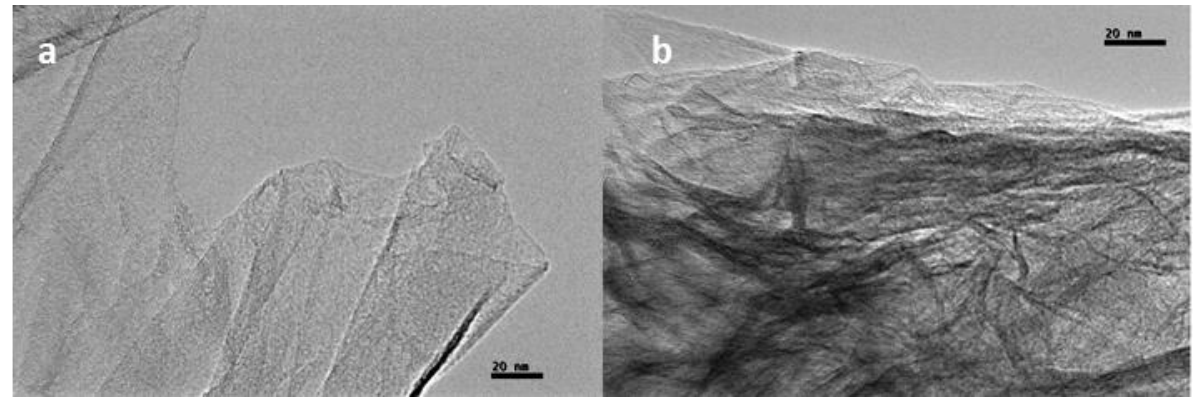

Figure 5.18 HRTEM images of $\mathbf{1 0 H}-$ rGO before (a) and after (b) six catalytic runs.

\subsubsection{NHC functionalised gold nanoparticles anchored on graphene}

\subsubsection{Synthesis and characterisation of ligand precursor I and metal complex 11 I}

In view of the important effect of the aromatic groups at the N-positions, a new imidazolium salt (I) was designed and synthesized. The new compound contains bulky 2,6-diisopropylphenyl groups (Dipp) at the $\mathrm{N}$-substituents and the pyrene group that acts as anchoring point onto $\mathrm{rGO}$ is connected through an ether functionality at the backbone of the NHC ring (Figure 5.19).

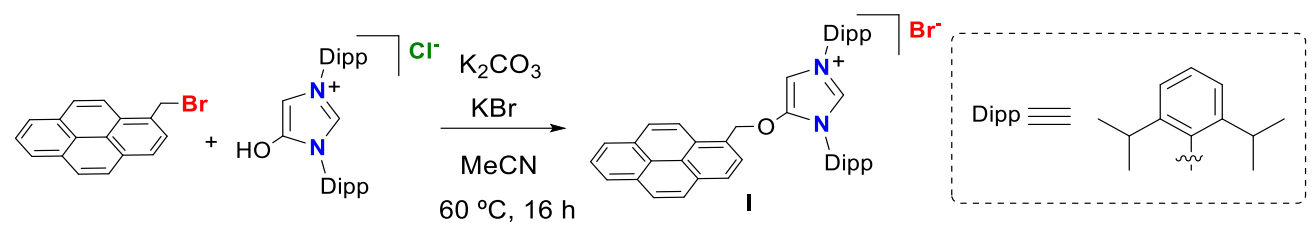

Figure 5.19 Synthesis of imidazolium salt I

The reaction in acetonitrile of 4-hydroxy-1,3-(2,6-diisopropylphenyl)imidazolium chloride ${ }^{[67]}$ with 1-(bromomethyl)pyrene in the presence of potassium carbonate as base affords compound I as a yellowish powder in $42 \%$ yield after crystallization in diethyl ether at $-18^{\circ} \mathrm{C}$. Compound I was characterised by NMR spectroscopy, ESI-MS and elemental analysis.

The ${ }^{1} \mathrm{H}$ NMR spectrum of I (Figure 5.20) shows a doublet at $9.54 \mathrm{ppm}$ due to the $\mathrm{NCHN}$ proton. This signal is coupled with the $\mathrm{CH}$ proton present at the backbone of 
the imidazole, which is shifted at 7.98 ppm. Between 8.26 and 8.06 the signals of the pyrene appear as a multiplet and the signals due to the protons of the aromatic groups at the $\mathrm{N}$-substituents are present between 7.50 and $7.30 \mathrm{ppm}$. In the aliphatic area the benzylic protons next to the pyrene group are shifted as a singlet at $6.09 \mathrm{ppm}$. The four $\mathrm{CH}$ protons of the four iso-propyl groups appear as two multiplets at 2.52 and $2.35 \mathrm{ppm}$, showing the unsymmetrical nature of the molecule. Finally, between 1.33 and 0.94 ppm there are four doublets due to the methyl protons of the iso-propyl groups.

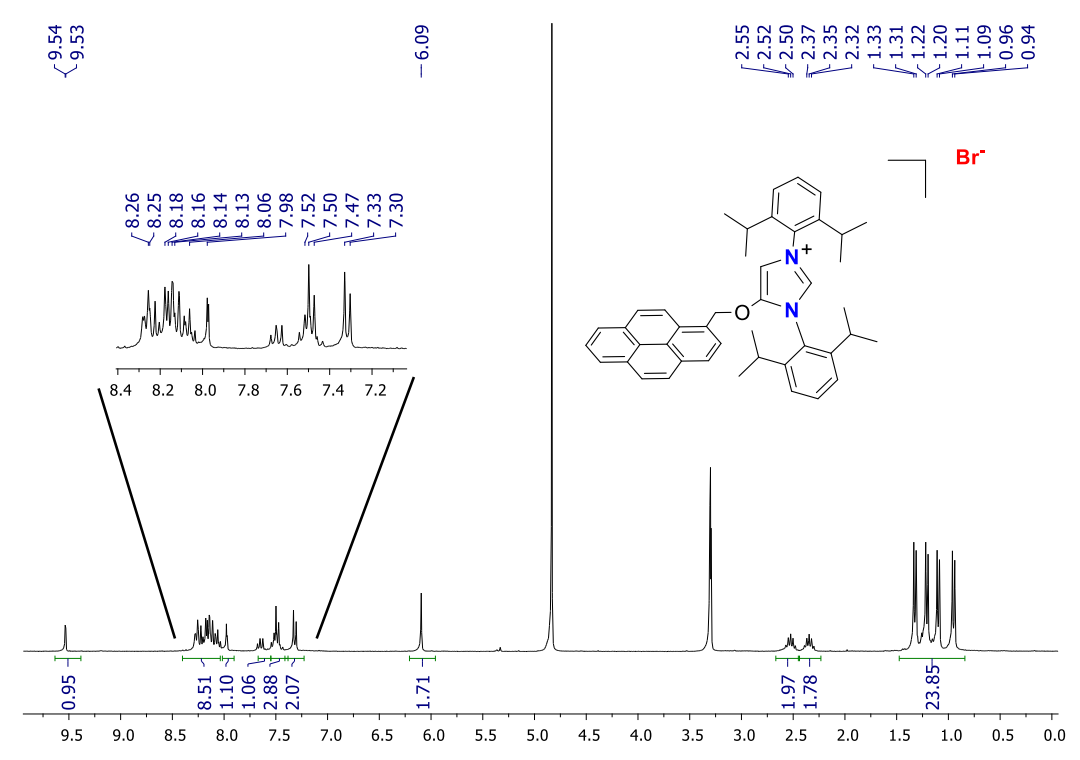

Figure $5.20^{1} \mathrm{H}$ NMR spectrum of compound I in MeOD

Figure 5.21 shows the ${ }^{13} \mathrm{C}\left\{{ }^{1} \mathrm{H}\right\}$ NMR spectrum of compound I. An HSQC spectrum was obtained in order to fully assign the signals in the ${ }^{13} \mathrm{C} N M R$ spectrum (see chapter 6). In brief, the signal due to the NCHN carbon is shifted at $133.3 \mathrm{ppm}$. The aromatic zone between 133.4 and $123.6 \mathrm{ppm}$ is complex and is not easy to distinguish which signals correspond with the pyrene tag or the aromatic groups at the $\mathrm{N}$-positions of the imidazole ring. The $\mathrm{CH}$ of the imidazole ring is shifted at 105.4 ppm and the benzylic $\mathrm{CH}_{2}$ appears at $74.9 \mathrm{ppm}$. In the aliphatic area the two different $\mathrm{CH}$ protons are present at 30.5 and $30.3 \mathrm{ppm}$ and the four signals due to the methyl groups are shown between 24.9 and 23.2 ppm. 


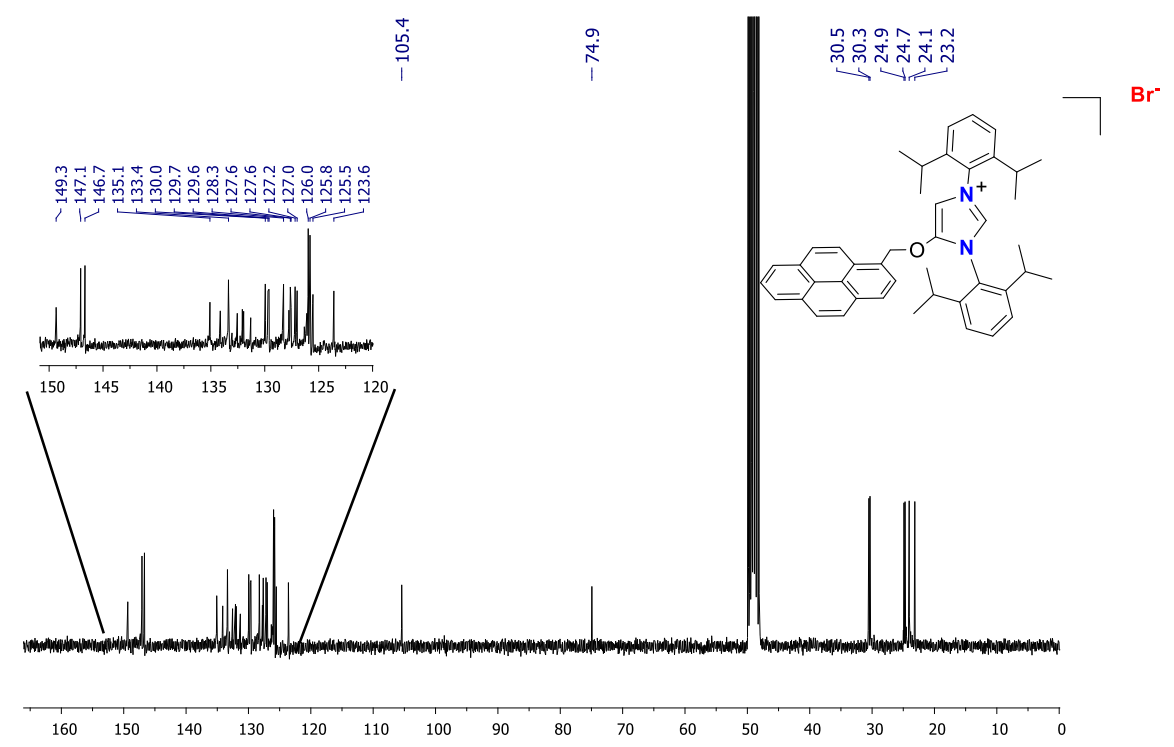

Figure $5.21{ }^{13} \mathrm{C}\left\{{ }^{1} \mathrm{H}\right\}$ NMR spectrum of compound $\mathrm{I}$ in MeOD

The mass spectrum of I shows a peak at a $\mathrm{m} / \mathrm{z}$ of 619.4 which is attributed to the $[\mathrm{M}-\mathrm{Br}]^{+}$fragment. This signal confirms the proposed molecular composition based on the mass/charge relation and the isotopic pattern.

The fully characterised imidazolium salt I was used as ligand precursor for the synthesis of the corresponding gold metal complex (Figure 5.22). Complex 11I was obtained by deprotonation of imidazolium salt I using potassium carbonate. Following the procedure reported by Nolan, ${ }^{[59]}$ imidazolium salt I, $\mathrm{AuCl}\left(\mathrm{SMe}_{2}\right)$ and potassium carbonate were suspended in $2 \mathrm{~mL}$ of acetone in a vial and the mixture was stirred at $60{ }^{\circ} \mathrm{C}$ for 18 hours. Complex 11 was obtained in a $66 \%$ yield after purification by column chromatography and recrystallization from dichloromethane/pentane.

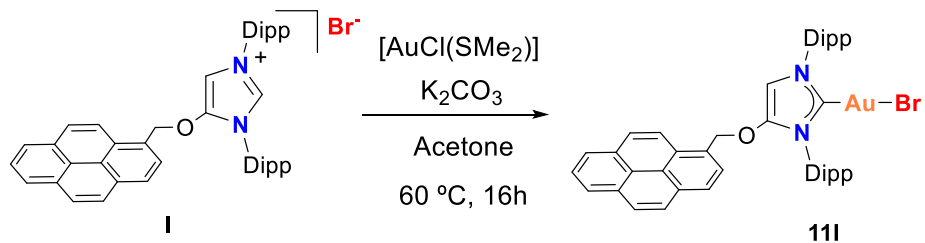

Figure $\mathbf{5 . 2 2}$ Synthesis of gold metal complex 11I 
The new gold compound was completely characterised by NMR, ESI-MS, elemental analysis and X-Ray diffraction. The ${ }^{1} \mathrm{H}$ NMR spectrum of 11l (Figure 5.23) shows the absence of the acidic signal of the NCHN proton, indicating that the metalation has taken place. Between 8.25 and $8.02 \mathrm{ppm}$ there are two doublets and one multiplet that correspond with the signals due to the pyrene. The signals due to the aromatic groups at the N-substituents appear between 7.46 and $7.21 \mathrm{ppm}$. At $6.42 \mathrm{ppm}$ there is a singlet attributed to the $\mathrm{CH}$ proton at the backbone of the imidazole ring; and the singlet due to the benzylic protons next to the pyrene appears at $5.74 \mathrm{ppm}$. The four $\mathrm{CH}$ protons of the four iso-propyl groups appear as one multiplet at $2.61 \mathrm{ppm}$. Finally, between 1.53 and $1.11 \mathrm{ppm}$ there are four doublets due to the methyl protons of the iso-propyl groups.

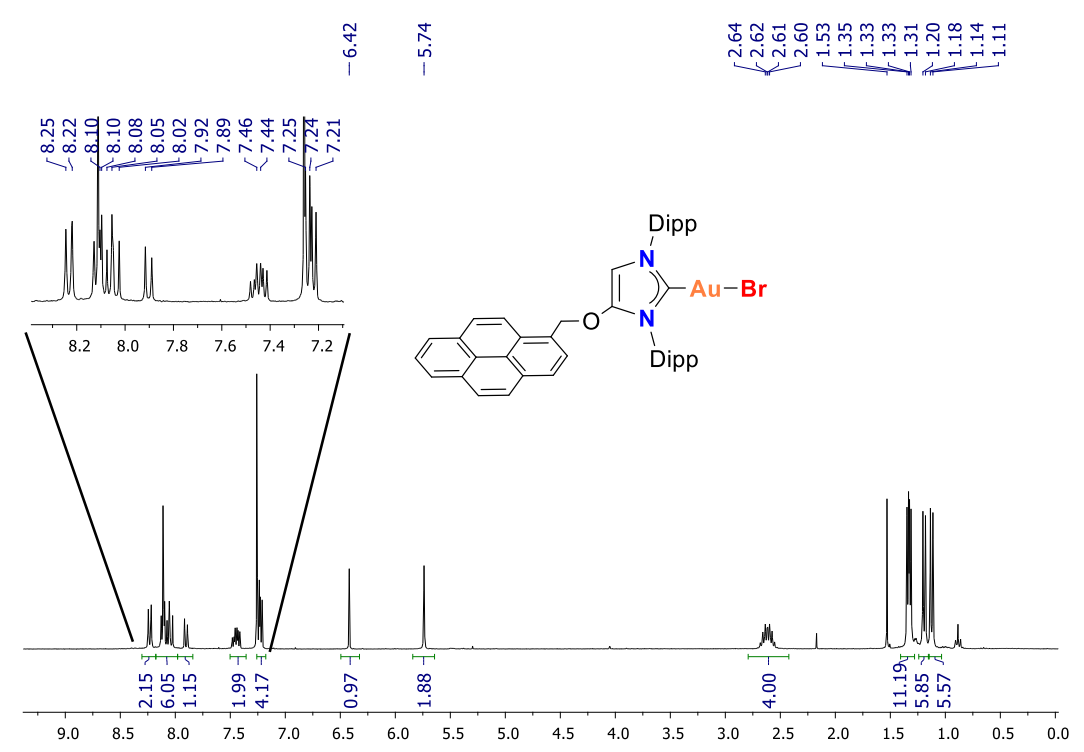

Figure $5.23{ }^{1} \mathrm{H}$ NMR spectrum of compound $11 \mathrm{l}$ in $\mathrm{CDCl}_{3}$

Figure 5.24 shows the ${ }^{13} \mathrm{C}\left\{{ }^{1} \mathrm{H}\right\}$ NMR spectrum of compound 11l. An HSQC spectrum was recorded in order to fully assign the signals in the ${ }^{13} \mathrm{C}$ NMR spectrum (see chapter 6). The most relevant signal is the one shifted at $173.8 \mathrm{ppm}$ that corresponds to the carbonic carbon and confirms the metalation. The resonance due to the carbon at the $C 5$ position of the imidazole ring appears at $101.1 \mathrm{ppm}$. The signal associated with the benzylic carbon next to the pyrene tag is shown at $73.3 \mathrm{ppm}$. Finally, the $\mathrm{CH}$ of the iso-propyl groups appear as two signals at 29.3 and $28.8 \mathrm{ppm}$, and the signals due to the methyl groups are shifted between 24.9 and $23.2 \mathrm{ppm}$. 


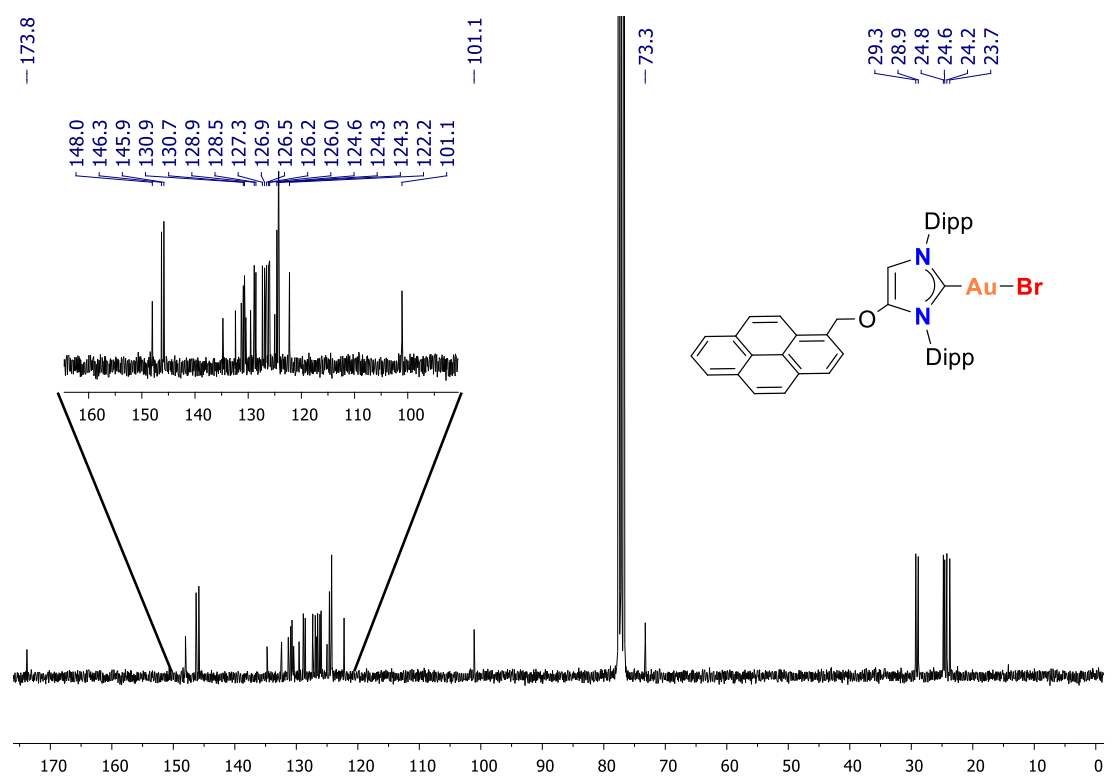

Figure $5.24{ }^{13} \mathrm{C}\left\{{ }^{1} \mathrm{H}\right\}$ NMR spectrum of 111 in $\mathrm{CDCl}_{3}$

Due to the strong nature of the $\mathrm{Au}-\mathrm{Br}$ bond that prevents bromine dissociation, the HRMS spectrum of $\mathbf{1 1 1}$ was recorded in the presence of potassium bromide to facilitate the formation of ionic species. Under these conditions the peak assigned to $[\mathrm{M}+\mathrm{K}]^{+}$was observed. Simulation of this peak confirms the formation of $\mathbf{1 1}$ on the basis of the mass/charge relation and isotopic pattern.

Crystals of 11I suitable for X-Ray diffraction analysis were grown by slow diffusion of hexane in a concentrated solution of the complex in dichloromethane (Figure 5.25). The Au- $\mathrm{C}_{\text {carbene }}$ distance is $1.982(5) \AA$. The coordination around the gold centre is linear with an angle of $177.7^{\circ}$. The bond distances and angles lie in the expected range when compared with other NHC-Au-Br complexes. ${ }^{[59]}$ 


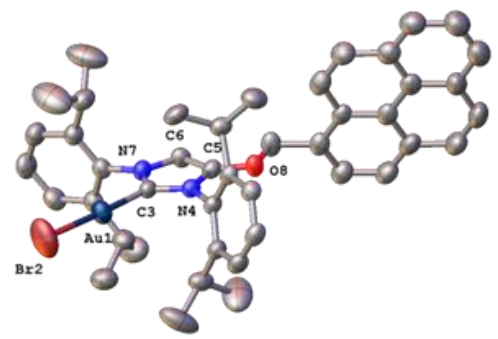

Figure 5.25 Molecular diagram of complex 11I. Ellipsoids are shown at $50 \%$ probability level. Hydrogens are omitted for clarity

\subsubsection{Direct generation and immobilisation of NHC-capped Au-NPs onto rGO}

Having the complex $\mathbf{1 1 l}$ in hand, the next step was the exchange of the bromine ligand for the more labile trifluoromethane sulfonate (OTf), which is very convenient in the design of homogeneous gold catalysts. ${ }^{[68]}$ Treatment of 11l with one equivalent of AgOTf in dichloromethane affords $\mathbf{1 2 I}$ after filtration over celite to remove insoluble $\mathrm{AgBr}$. The remaining solution was treated with a suspension of $\mathrm{rGO}$ in dichloromethane for two days at room temperature and under magnetic stirring. The resulting solid was filtered and washed thoroughly with dichloromethane, affording the final product as a black solid (Figure 5.26).

We have observed that the presence of two bulky aromatic groups is essential to obtain $\mathbf{1 2 I}$ as an stable complex. When $\mathbf{1 0 H}$ was treated with AgOTf, the formation of the related NHC-Au-OTf was observed, however rapid degradation of the complex took place as evidenced by NMR spectroscopy. These results show the importance of ligand design in developing gold catalysts.

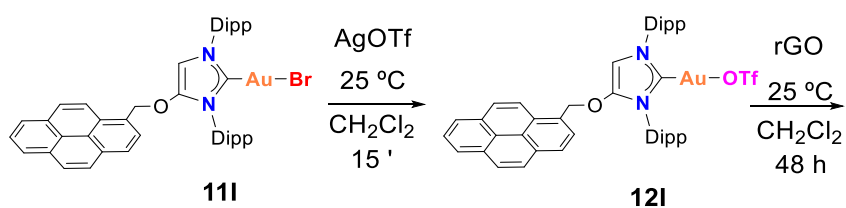

121

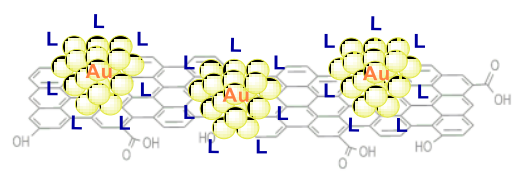

12I-rGO-NPs

Figure 5.26 Synthesis of 12I-rGO-NPs

Analysis of the new material shows that the immobilisation of $\mathbf{1 2 I}$ on graphene gives completely different results to our previous results using $\mathbf{1 0 H}$ (section 5.2.1.2). The NHC-Au-OTf complex is stable in solution and in the solid state. However, in the presence of rGO complex $\mathbf{1 2 I}$ decomposes and generates gold nanoparticles 
(Au-NPs) that are directly immobilised on the surface of rGO. These Au-NPs are generated under mild reaction conditions and in the absence of any reducing agent. Thus, it is expected that the properties of the rGO are maintained.

Analysis by TEM reveals the presence of small nanoparticles with a quasi-spherical shape. Interestingly, these nanoparticles are highly crystalline (Figure 5.27, a and b). The reticular distance between planes is $2.3 \AA$, which corresponds with the $\left[\begin{array}{lll}1 & 1 & 1\end{array}\right]$ plane of the fcc packing of gold, as previously reported. ${ }^{[69]}$ A particle-size histogram was obtained revealing an average diameter of $3.2 \pm 1.5 \mathrm{~nm}(n=450)$ (Figure 5.27, c). When the time of the interaction between rGO and $\mathbf{1 2 I}$ was increased from 48 to $72 \mathrm{~h}$ no increase in particle size was observed. These results indicate that graphene induces the formation of small spherical nanoparticles and acts as stabiliser, preventing particle growth. Our group has previously demonstrated that graphene stabilises Pd-NPs, controlling its size and morphology. ${ }^{[39]}$
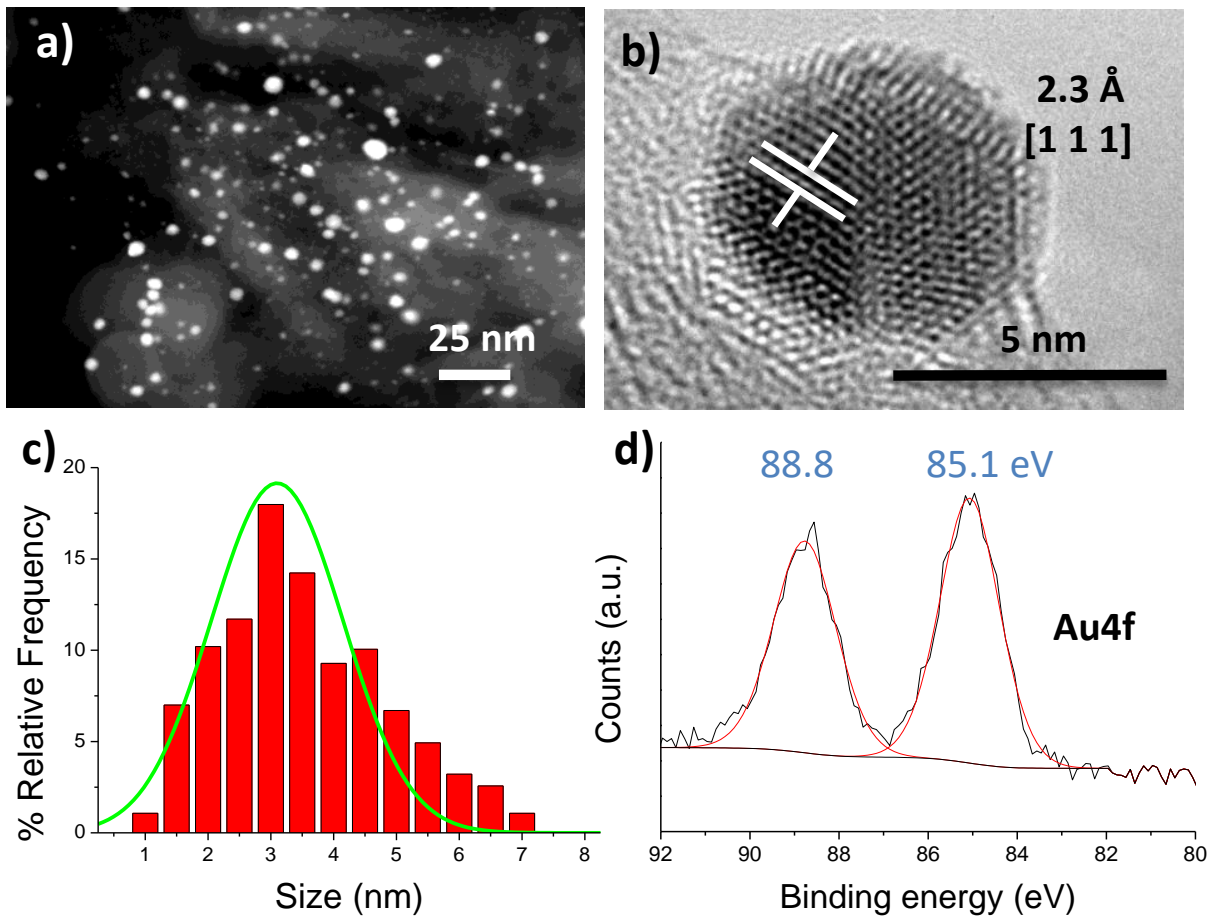

Figure 5.27 Characterisation of gold nanoparticles on rGO (12I-rGO-NPs). a) HAADF micrograph showing spherical AuNPs onto graphene. b) HRTEM micrographs showing the interplanar spacing. c) Size distributtion histogram of the AuNPs $(n=450)$ d) XPS analysis of the core-level peaks (eV) for the Au4f region of 12I-rGO-NPs 
Assignment of the core-level peaks of $\mathrm{N}(400.7 \mathrm{eV})$ and $\mathrm{F}(688.0 \mathrm{eV})$ in the XPS spectra confirm the presence of NHC and OTf ligands on the surface of the nanoparticle. The Au4f region shows a doublet peak (due to the two transitions $4 f_{7 / 2}$ and $4 \mathrm{f}_{5 / 2}$ ) at a binding energy of 88.8 and $85.1 \mathrm{eV}$ (Figure 5.27, d). These values correlate well with the presence of $A u(I)$ at the surface of the Au-NPs, but no Au (0) could be detected. Nevertheless, these results and are in agreement with a recent report by Toste and Somorjai. ${ }^{[58]}$ They observed that Au-NPs functionalised with chiral NHC ligands show only the presence of $A u(I)$ at the surface of the NP by XPS. Further characterisation by X-Ray Absorption Near Edge Structure (XANES) spectroscopy confirm the presence of $95 \%$ of $\mathrm{Au}(0)$ at the core. 12I-rGO-NPs was further analysed by UV/Vis, showing the characteristic plasmon resonance of gold nanoparticles at $510 \mathrm{~nm}$ (Chapter 6, Figure 6.27).

As a control experiment, complex 11l was immobilised on rGO using the same reaction conditions. Analysis by HRTEM shows the absence of metal nanoparticles, and EDS analysis confirms the presence of gold on the surface of rGO (see chapter $6)$. These results indicate that molecular $\mathbf{1 1 I}$ is grafted at the surface of rGO and that the presence of the more labile OTf counteranion is necessary to produce the complex decomposition into metal nanoparticles.

For comparative purposes, ligandless gold nanoparticles were synthesized from the commercially available $\mathrm{HAuCl}_{4}{ }^{[70]}$ Reduced graphene oxide was exfoliated by sonicating a suspension of $\mathrm{rGO}$ in dichloromethane for 30 minutes. Then, $\mathrm{HAuCl}_{4}$ was added and the mixture was magnetically stirred for 48 hours, affording $\mathrm{HAuCl}_{4}$-rGO-NPs as a black solid. Characterisation by HRTEM confirms the formation of gold nanoparticles on the surface of graphene. The histogram (Figure 5.28, b) shows a broad particle size distribution with an average size of $12.4 \pm 4.2 \mathrm{~nm}$ ( $n=74$ ), which results in an increased particle size of $9.2 \mathrm{~nm}$ with respect 12I-rGO-NPs. These observations indicate that NHC ligand has an important effect in determining the final size of the nanoparticle. In the absence of ligands bigger gold nanoparticles are formed with a heterogeneous distribution of particle size, which may affect the catalytic properties. 


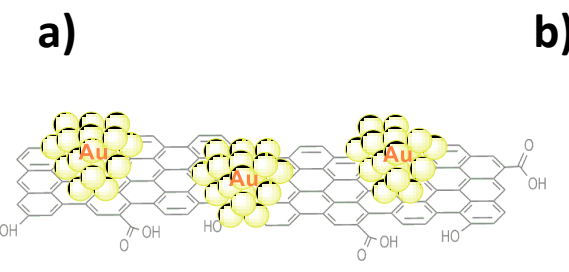

$\mathrm{HAuCl}_{4}$-rGO-NPs

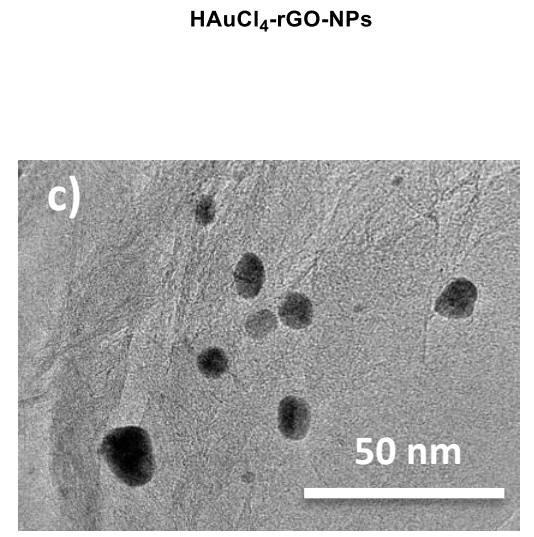

b)
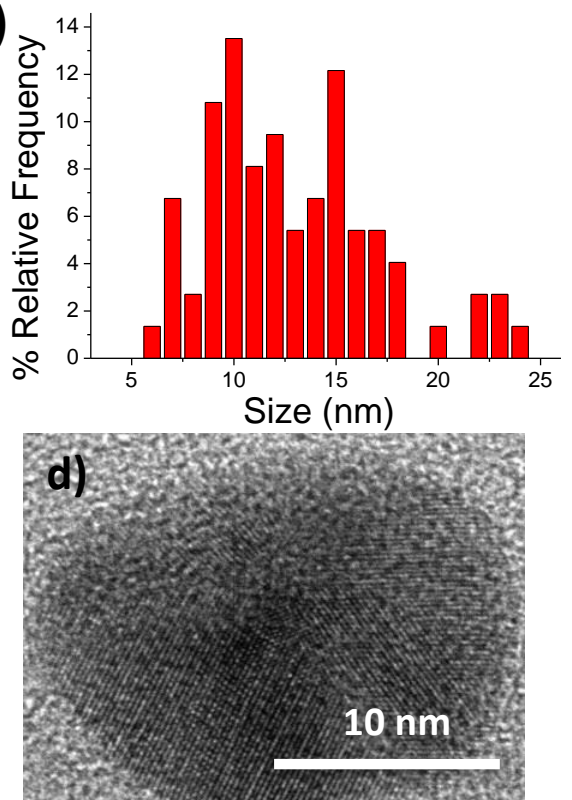

Figure 5.28 Characterisation of Au-NPs prepared from $\mathrm{HAuCl}_{4}$. a) Schematic representation of ligandless Au-NPs. b) Size distribution histogram of $\mathrm{HAuCl}_{4}-\mathbf{r G O}-\mathbf{N P s}$. c) and d) HRTEM micrographs

\subsubsection{Catalytic properties of 12I, 12I-rGO-NPs and HAUCl4-rGO-NPs}

The catalytic properties of the new synthesized compounds were tested in the hydration of alkynes to obtain ketones. The optimization of the reaction conditions was carried out using 4-octyne as model substrate (Table 5.2).

The results showed that a catalyst is needed to carry out the hydration of alkynes (entry 1). Blank experiments with $\mathrm{rGO}$ and silver triflate reveal that these compounds are not active (entries 2 and 3). $\mathrm{HAuCl}_{4}$ has a low activity and, as expected, compound $\mathbf{1 1}$ is completely inactive in the hydration of alkynes (entries 4-5). Addition of AgOTf causes halide exchange and generates the active species $\mathbf{1 2 1}$. At a catalyst loading of $0.25 \mathrm{~mol} \%$ complex $\mathbf{1 2 I}$ affords quantitative conversion in 3 hours (entry 6). Higher activity is observed with 12I-rGO-NPs. Using a catalyst loading of $0.05 \mathrm{~mol} \%$ the reaction is complete in just 1 hour (entry 7). Interestingly, the gold nanoparticles obtained from tetrachloroauric acid are completely inactive under the same reaction conditions (entry 10) probably because of the bigger average size of the nanoparticles. In global, the results contained in Table 5.2 show the positive effect of graphene and ligands in the generation of highly active catalysts based on Au-NPs. 
Table 5.2 Optimisation of the reaction conditions

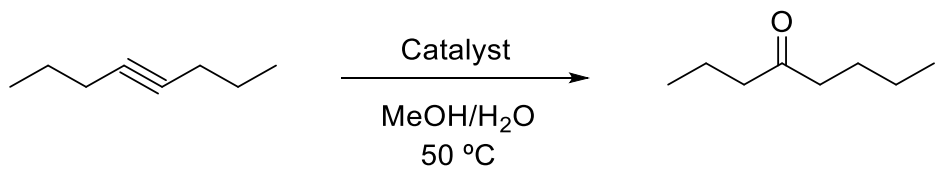

\begin{tabular}{|c|c|c|c|c|}
\hline Entry & Catalyst & Loading (mol \%) & Time (h) & Conversion (\%) \\
\hline 1 & - & - & 24 & 0 \\
\hline 2 & rGO & $60 \mathrm{mg}$ & 24 & 0 \\
\hline 3 & AgOTf & 5 & 24 & 0 \\
\hline 4 & $\mathrm{HAuCl}_{4}$ & 0.25 & 24 & 18 \\
\hline 5 & 111 & 0.25 & 24 & 0 \\
\hline 6 & 121 & 0.25 & 3 & 100 \\
\hline 7 & 12I-rGO-NPs & 0.05 & 1 & $100(97)$ \\
\hline 8 & 12I-rGO-NPs & 0.0025 & 72 & 100 \\
\hline 9 & 12I-rGO-NPs & 0.0005 & 375 & 56 \\
\hline 10 & $\mathrm{HAuCL}_{4}$-rGO-NPS & 0.025 & 24 & 0 \\
\hline
\end{tabular}

12I was generated in situ by treatment of 11 l with 1.1 eq. of AgOTf. [a] Conversions determined by GC-FID. Isolated yields in parenthesis.

With the optimised conditions in hand, the catalyst scope was investigated. Both 12I and 12I-rGO-NPs are competent catalysts in the hydration of alkylic and aromatic alkynes, terminal and internal. The results indicate that catalyst 12I-rGO-NPs is very active at low catalyst loadings, affording complete conversions and high turnover frequencies (TOFs) in short reaction times. On the contrary, the molecular complex 12I usually requires a tenfold catalyst loading to reach similar conversion, but TOFs are systematically lower (Table 5.3). 
Table 5.3 Reaction scope for the hydration of alkynes
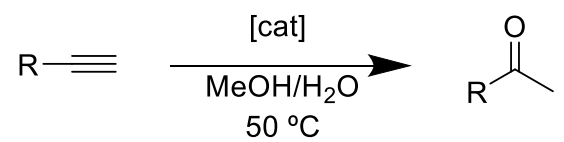

Code: Catalyst (loading)

Yield \% (time) TOF $\left(h^{-1}\right)$<smiles>CCCCC(C)=O</smiles>

12I rGO-NPs $(0.02 \mathrm{~mol} \%)$

$97 \%(180 \mathrm{~min})$

TOF $=2343 \mathrm{~h}^{-1}$

$121(0.25 \mathrm{~mol} \%)$

$95 \%$ (150 min)

TOF $=344 \mathrm{~h}^{-1}$<smiles>CCCCC(=O)CCC</smiles>

12I rGO-NPs $(0.05 \mathrm{~mol} \%)$

$100 \%(60 \mathrm{~min})$

TOF $=2000 \mathrm{~h}^{-1}$

$$
\begin{aligned}
& 12 \mathrm{l}(0.25 \mathrm{~mol} \%) \\
& 100 \%(180 \mathrm{~min}) \\
& \text { TOF }=320 \mathrm{~h}^{-1}
\end{aligned}
$$<smiles>CC(=O)c1ccccc1</smiles>

12I- rGO-NPs $(0.05 \mathrm{~mol} \%)$ $100 \%$ (120 min)

TOF $=1314 \mathrm{~h}^{-1}$

12I $(0.5 \mathrm{~mol} \%)$

$97 \%$ (240 min)

TOF $=90 \mathrm{~h}^{-1}$<smiles>CC(=O)CCCC#N</smiles>

12I rGO-NPs (0.02 mol \%) $100 \%$ (500 min)

TOF $=581 \mathrm{~h}^{-1}$

$12 \mathrm{l}(0.25 \mathrm{~mol} \%)$

$100 \%$ (400 min)

TOF $=116 \mathrm{~h}^{-1}$<smiles>CC(=O)c1ccc(C)cc1</smiles>

121 rGO-NPs (0.02 mol \%) $100 \%$ (400 min)

TOF $=622 \mathrm{~h}^{-1}$

$121(0.25 \mathrm{~mol} \%)$

$98 \%$ (200 min)

TOF $=180 \mathrm{~h}^{-1}$<smiles>CCC(=O)c1ccccc1</smiles>

12I rGO-NPs (0.05 mol \%) $96 \%$ (150 min)

TOF $=900 \mathrm{~h}^{-1}$

$121(0.25 \mathrm{~mol} \%)$

$98 \%$ (300 min)

TOF $=154 \mathrm{~h}^{-1}$

Reaction conditions: alkyne $(0.2$ or $0.5 \mathrm{mmol}), \mathrm{MeOH}\left(0.142 \mathrm{M}\right.$ in alkyne) and 2 eq. of water at $50^{\circ} \mathrm{C}$. Yields calculated by ${ }^{1} \mathrm{H}$ NMR using 1,3,5-trimethoxybenzene as an internal standard. TOF values calculated at $1 \mathrm{~h}$ reaction.

As an example of the superior activity of 12I-rGO-NPs vs 121, the reaction monitoring profile for the hydration of 4-octyne was performed using both catalysts (Figure 5.29). While 12I-rGO-NPs affords quantitative yield in $60 \mathrm{~min}$, complex 121 needs $180 \mathrm{~min}$ to reach a $100 \%$ yield, but requires to increase the catalyst loading up to $0.25 \mathrm{~mol} \%$. This effect is general, the reaction monitoring for all the substrates 
contained in Table 5.3 was carried out showing always faster rates when using 12I-rGO-NPs.
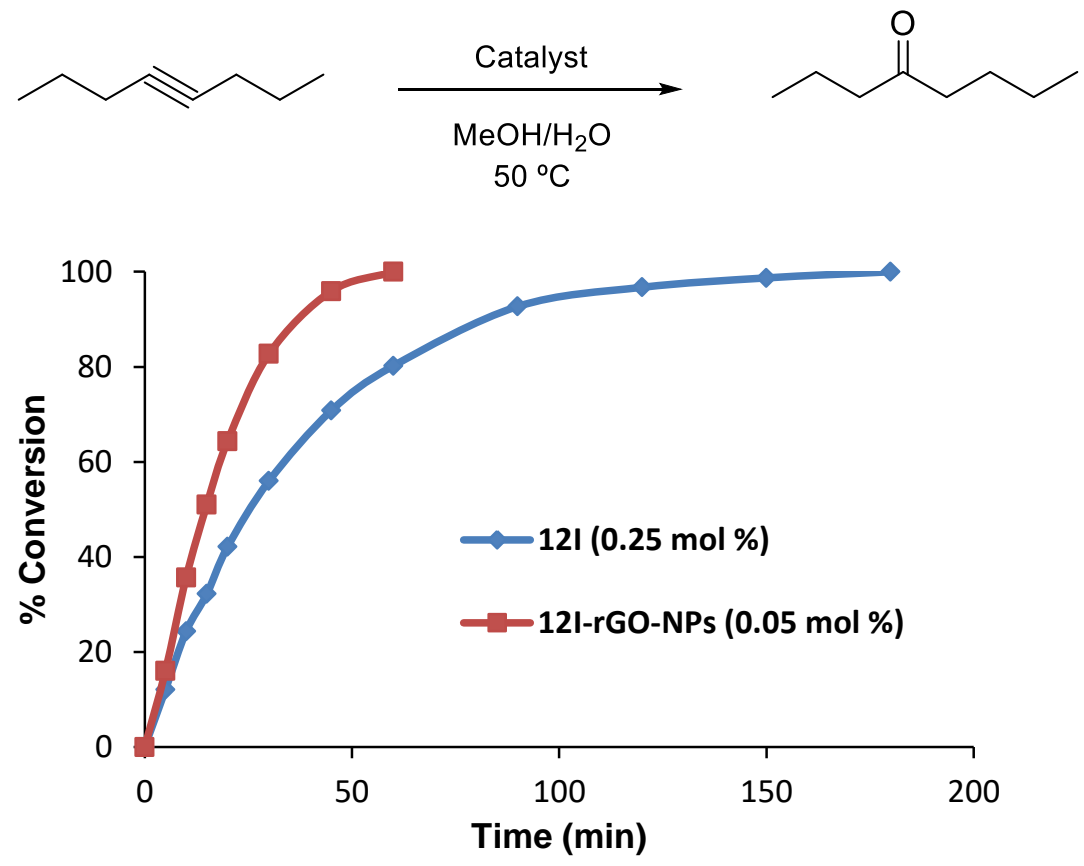

Figure 5.29 Reaction monitoring profile of $\mathbf{1 2 I}$ vs $\mathbf{1 2 1 - r G O - N P s ~ i n ~ t h e ~ h y d r a t i o n ~ o f ~ 4 - o c t y n e ~}$

To determine the nature of the active species when 121 was used as catalyst, poisoning experiments were carried out. The hydration of phenyl acetylene was performed in the presence of $\mathrm{Hg}$, a poison for metal nanoparticles, or poly(4-vinylpyridine) (PVP), a polymer that strongly bond to molecular complexes and turn them inactive. Experimentally, the hydration of phenyl acetylene under standard reaction conditions was set up in three parallel experiments. After 90 min, $\mathrm{Hg}$ (300 eq.) was added to one reaction, PVP to the second one, and the remaining was used as control (Figure 5.30). The results show that mercury does not affect the kinetics of the reaction but the PVP completely inhibits the reaction. Thus, the poisoning experiments indicate that no metal nanoparticles are in the media when $12 \mathrm{I}$ is the catalyst of choice. These results are in accordance with the experimental observation of the absence of an induction time when complex $\mathbf{1 2 l}$ is used. The completely inhibition of the reaction upon addition of PVP indicates that a molecular entity is the active catalytic species when $\mathbf{1 2 I}$ is used as the (pre)catalyst. 
Cat. 12I
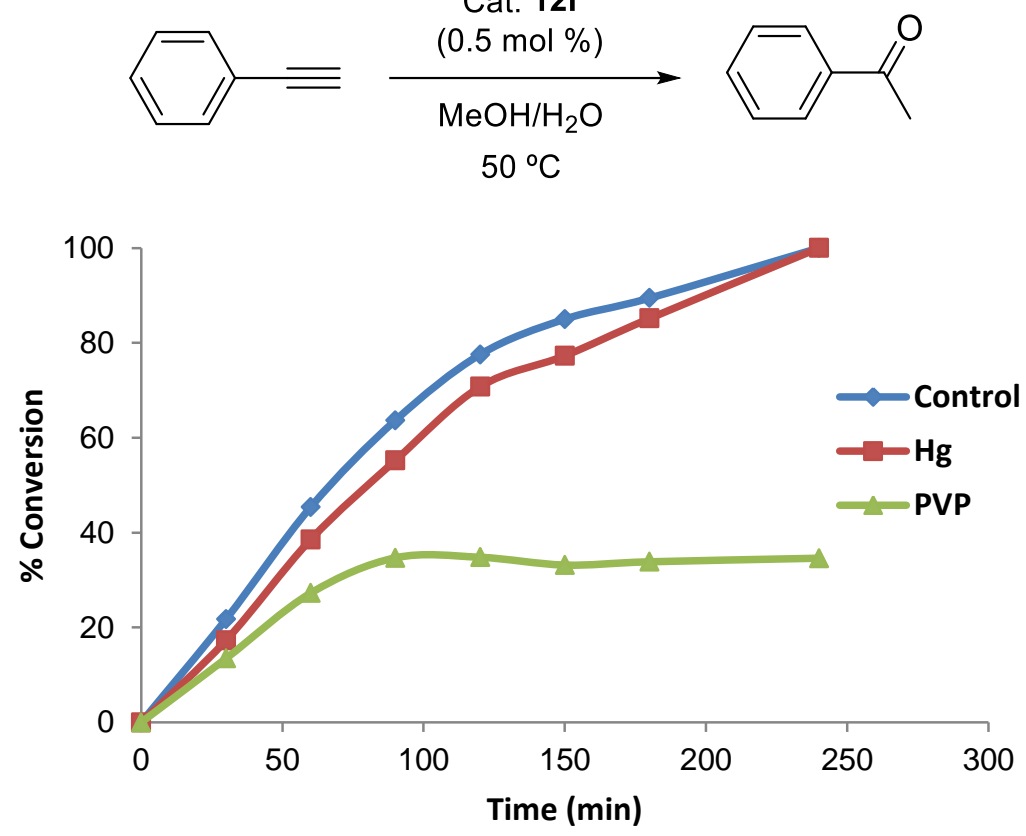

Figure 5.30 Poisoning experiments using $\mathbf{1 2 I}$ as catalyst

In the case of catalyst 12I-rGO-NPs, the hot filtration test was performed to analyse the presence of any leached species during the catalysis (Figure 5.31). The hydration of 4-octyne was set up under the standard conditions (Table 5.2, entry 7) and, after 10 min (GC conversion $38 \%$ ) half of the reaction mixture was separated by filtration at $50{ }^{\circ} \mathrm{C}$. The filtrate and the reaction mixture (which contained the solid catalyst) were maintained for 300 min under the reaction conditions. Analysis by GC showed that in the filtrate the conversion was almost unaltered after 50 min (GC conversion $41 \%$ ). However, after extended time (300 $\mathrm{min}$ ) a few more hydration occurred (GC conversion $53 \%$ ). In parallel, the remaining solid catalyst afforded full conversion in less than 50 min (Figure 5.31). These results confirm the heterogeneous nature of the catalyst, with few leaching taking place during the reaction. 


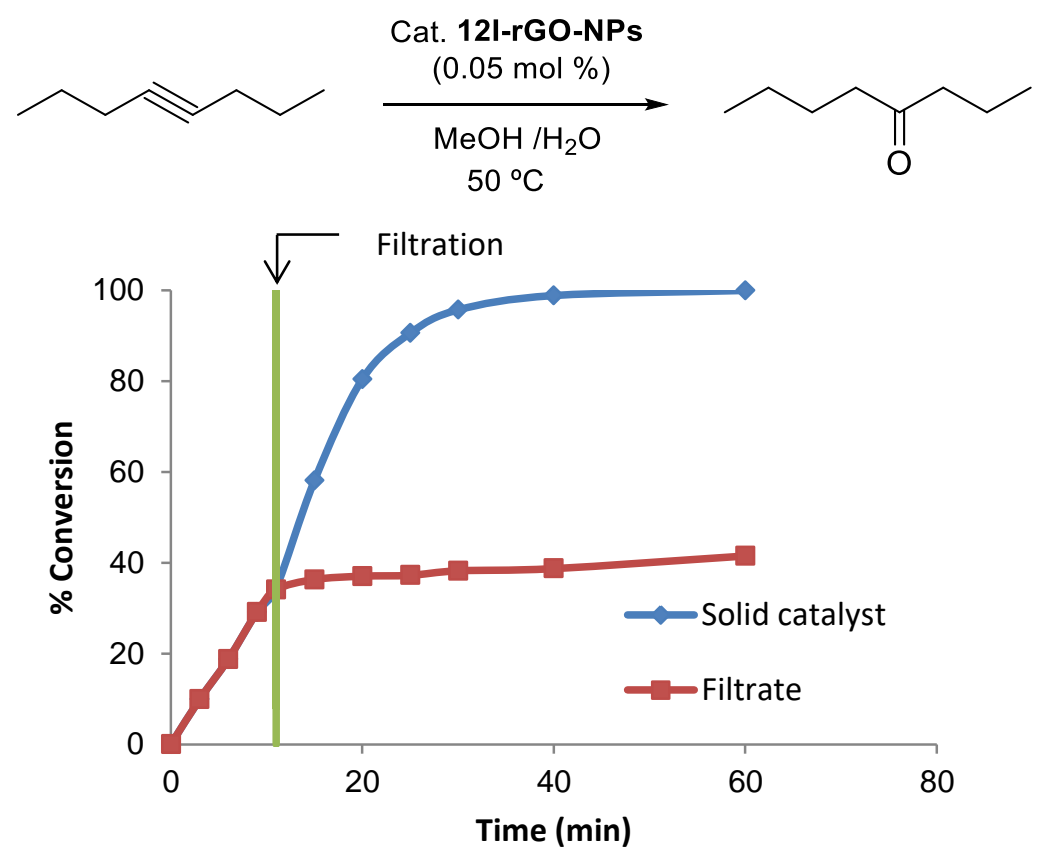

Figure 5.31 Reaction profiles for the hot filtration test experiment

Then, the recycling properties of 121-rGO-NPs were studied using 4-octyne as the substrate (Figure 5.32). The reaction was set up in $\mathrm{MeOH}$ using a catalyst loading of $0.05 \mathrm{~mol} \%$ of 12I-rGO-NPs and 2 equivalents of water, and the mixture was heated at $50{ }^{\circ} \mathrm{C}$ until full conversion was observed. After each run the catalyst was separated by centrifugation, washed with methanol, dried with pentane and reused. Under these conditions, the catalyst 12I-rGO-NPs was reused three times without any decrease in activity. In runs four to eight some deactivation took place, but complete conversion was obtained in short reaction times ( $100 \mathrm{~min}$ for runs 4 and 5 , 150 min for runs 6 and 7 and 200 min for run 8). After the eighth run the reaction rate further decreased, however quantitative yields were obtained in run ten after 400 min. 

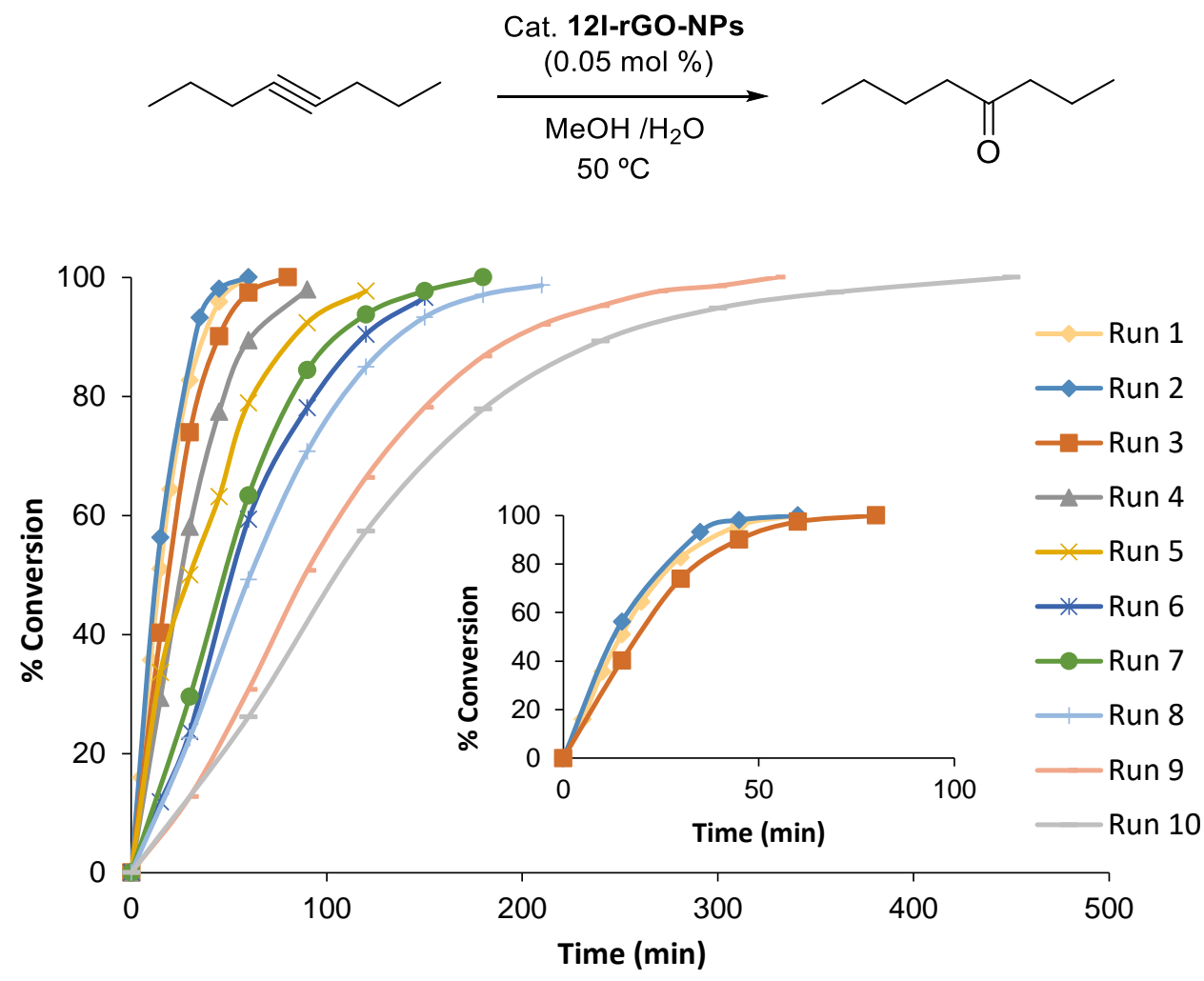

Figure 5.32 Reaction profile of 12I-rGO-NPs in the hydration of 4-octyne for 10 consecutive runs

To elucidate the deactivation mechanism, the solid catalyst was studied by HRTEM and XPS at the end of the recycling experiments. HRTEM images of 121-rGO-NPs before and after ten catalytic runs show that the morphology of the graphene is maintained after the recycling experiments. The support has not suffered any alteration and gold nanoparticles are homogeneously distributed along the graphene sheets (Figure 5.33, a). In addition, the nanoparticles preserve the high crystallinity even after 10 reaction cycles (Figure 5.33, b). A size-distribution histogram after the tenth run shows a mean size of $2.5 \pm 1.1 \mathrm{~nm}$, indicating that no agglomeration occurs during the catalysis (Figure $5.33, \mathrm{c}$ ). This observation discards particle growth as the deactivation mechanism. In addition, the XPS spectrum of the Au4f region (Figure 5.33, d) show the same doublet peak at very similar binding energies ( 88.6 and $84.9 \mathrm{eV}$ after the catalysis vs 88.8 and $85.1 \mathrm{eV}$ before the recycling experiment). 

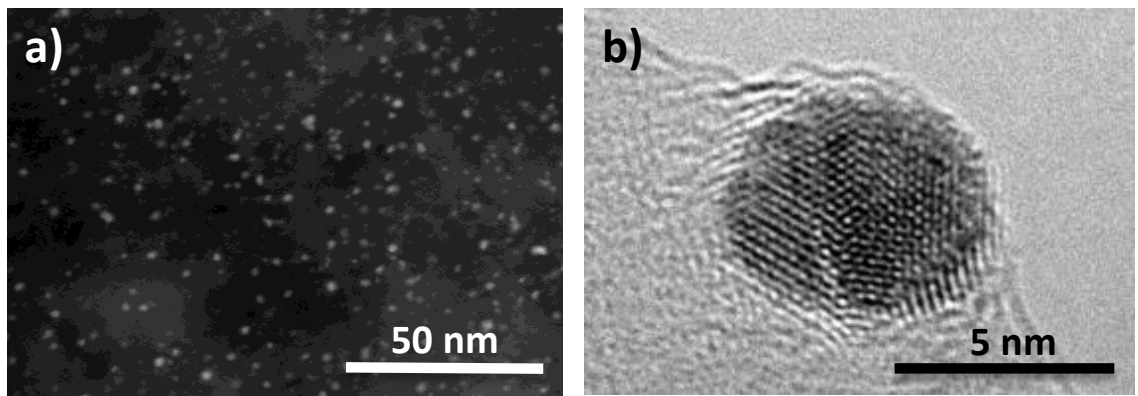

\section{c)}
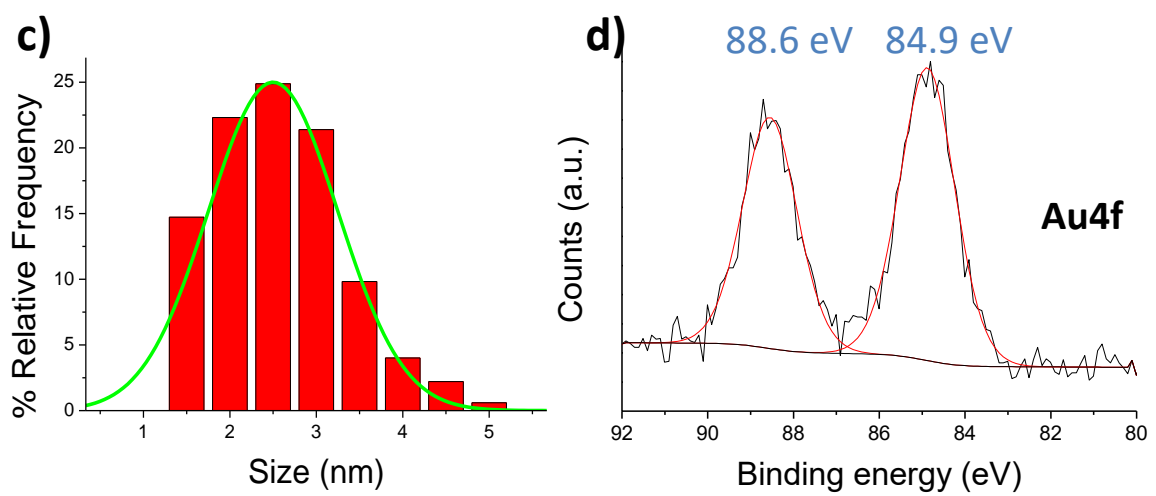

Figure 5.33 a) Dark field TEM micrograph showing small Au-NPs on the graphene after the $10^{\text {th }}$ run. b) HRTEM micrograph showing the good crystallinity of the nanoparticles after the $10^{\text {th }}$ run. c) Size distribution histogram after 10 catalytic cycles. $(n=292)$. d) XPS analysis of the core-level peaks (eV) for the Au4f region after the recycling experiment

To study if metal leaching was implied in the deactivation mechanism, the gold content in the filtrate after each run was analysed by ICP-MS. The results reveal that the accumulated metal content in the solution for runs 1 to 3 is $15 \%$, which correlates with the low change in activity. In runs four and five the accumulated metal leaching increases up to $30 \%$. At the end of the recycling experiment the metal content in solution was around $50 \%$ of the initial metal content in the solid, which explain the decay in activity of 12I-rGO-NPs. At the end of the ten runs the solid catalyst was also analysed by ICP-MS. The results show that the metal loading has decreased $50 \%$, showing a perfect match for the results of metal content in the solution and in the solid after the catalysis (Table 5.4).

Table 5.4 Results of ICP-MS studies after the recycling experiment

\begin{tabular}{|c|c|c|}
\cline { 2 - 3 } \multicolumn{1}{c|}{} & mg of gold & $\%$ initial gold \\
\hline 12I-rGO-NPs before catalysis & 0,02025 & $100 \%$ \\
\hline 12I-rGO-NPs after catalysis & 0,01125 & $55.5 \%$ \\
\hline lixiviate after ten runs & 0,01048 & $51.7 \%$ \\
\hline
\end{tabular}


In global, the study of the catalyst after the recycling experiment shows that:

- $\quad$ Agglomeration of nanoparticles does not take place during catalysis

- rGO favours the stabilisation of nanoparticles

- Metal leaching is the cause of catalyst deactivation

\subsection{CONCLUSIONS}

The preparation of NHC-Au-X (X= $\mathrm{Br}$ or $\mathrm{Cl}$ ) complexes anchored on reduced graphene oxide by $\pi-\pi$ interactions has been described. The characterisation of the new hybrid materials show that the catalyst is composed by a molecular gold complex grafted onto reduced graphene oxide. No nanoparticles are formed and XPS analysis reveal the presence of the molecular compound onto rGO.

The catalytic properties of gold complexes and the related hybrid materials have been studied in the intramolecular hydroamination reaction. The results show the important influence of ligand design. Introducing an aromatic group (instead of one methyl) at one of the $\mathrm{N}$-substituents of the imidazolylidene ring has a dramatic effect in the catalytic activity.

An increase in the catalytic activity has been also observed when the molecular complexes were grafted on rGO. The hybrid materials show faster reaction rates than the corresponding molecular complexes. Usually anchoring molecular catalysts on solid supports have a negative effect due to diffusion problems. In this case the 2D nature of graphene provides easy access to the active centres and may stabilise the active species, inhibiting the decomposition pathways. In addition, the use of graphene allows the easy recyclability of the catalyst for six consecutive runs with complete conversions in short reaction times.

The second part of this chapter shows a new methodology for the generation of highly active NHC-capped Au NPs. Anion exchange from a halide to a triflate cause a dramatic change when the complex is treated with rGO. Stirring a NHC-Au-OTf complex in a suspension of $\mathrm{rGO}$ produces the generation of NHC-functionalised gold nanoparticles anchored on graphene in one-step and in the absence of any reducing agent. Morphological analysis show the formation of small Au-NPs and XPS demonstrates the presence of ligands at the surface of the nanoparticles.

The new hybrid catalyst has been tested in the hydration of alkynes in the absence of additives. The results show that the new platform is a more active catalyst than the parent molecular complex. Internal and terminal alkynes can be activated at very 
low catalyst loadings (up to $0.0025 \mathrm{~mol} \%$ ) affording complete conversions and high TOFs.

The catalytic system is active in consecutive reaction cycles. In particular the catalyst can be recycled 3 times without any decrease in activity. Then the reaction rate gradually decreases, but quantitative yields are obtained for seven more runs. Analysis by HRTEM and XPS show that the morphology, structure and size of metal nanoparticles remain unchanged after the catalysis. Studies by ICP-MS indicate that the deactivation mechanism is mainly governed by metal leaching. These experiments show that graphene plays an essential role in the formation, and stabilisation of metal nanoparticles. The presence of NHC ligands in combination with graphene constitutes a new approach to develop novel hybrid materials for catalytic applications. 


\subsection{REFERENCES}

[1] A. Corma, H. Garcia, Chem. Soc. Rev. 2008, 37, 2096-2126.

[2] J. Oliver-Meseguer, J. R. Cabrero-Antonino, I. Domínguez, A. Leyva-Pérez, A. Corma, Science 2012, 338, 1452-1455.

[3] D. J. Gorin, B. D. Sherry, F. D. Toste, Chem. Rev. 2008, 108, 3351-3378.

[4] A. S. K. Hashmi, Angew. Chemie - Int. Ed. 2010, 49, 5232-5241.

[5] W. Wang, G. B. Hammond, B. Xu, J. Am. Chem. Soc. 2012, 134, 5697-5705.

[6] N. Marion, R. S. Ramón, S. P. Nolan, J. Am. Chem. Soc. 2009, 131, 448-449.

[7] Y. Wang, Z. Wang, Y. Li, G. Wu, Z. Cao, L. Zhang, Nat. Commun. 2014, 5, 34703477.

[8] V. Belting, N. Krause, Org. Lett. 2006, 8, 4489-4492.

[9] X. Han, R. A. Widenhoefer, Angew. Chemie - Int. Ed. 2006, 118, 1779-1781.

[10] A. S. K. Hashmi, T. M. Frost, J. W. Bats, J. Am. Chem. Soc. 2000, 122, 1155311554.

[11] W. Frey, A. S. K. Hashmi, M. C. Blanco, E. Kurpejovic, J. W. Bats, Adv. Synth. Catal. 2006, 348, 709-713.

[12] A. S. K. Hashmi, Chem. Rev. 2007, 107, 3180-3211.

[13] A. Corma, P. Serna, Science 2006, 313, 332-334.

[14] S. Carrettin, P. McMorn, P. Johnston, K. Griffin, G. J. Hutchings, Chem. Commun. 2002, 7, 696-697.

[15] M. D. Hughes, Y. Xu, P. Jenkins, P. Mcmorn, P. Landon, D. I. Enache, A. F. Carley, G. A. Attard, G. J. Hutchings, F. King, et al., Nature 2005, 437, 11321135.

[16] A. Corma, P. Concepcion, M. Boronat, M. J. Sabater, J. Navas, M. J. Yacaman, E. Larios, A. Posadas, M. A. López-Quintela, D. Buceta, et al., Nat. Chem. 2013, $5,775-781$.

[17] A. Corma, A. Leyva-Pérez, M. J. Sabater, Chem. Rev. 2011, 111, 1657-1712.

[18] A. Arcadi, Chem. Rev. 2008, 108, 3266-3325. 
[19] F. Zhu, F. Zhang, X. Yang, J. Huang, H. Li, J. Mol. Catal. A Chem. 2011, 336, 17.

[20] D. Liu, Q. Nie, R. Zhang, M. Cai, Tetrahedron Lett. 2019, 60, 29-34.

[21] A. Corma, E. Gutiérrez-Puebla, M. Iglesias, A. Monge, S. Pérez-ferreras, F. Sánchez, Adv. Synth. Catal. 2006, 348, 1899-1907.

[22] C. Pozo, A. Corma, M. Iglesias, F. Sánchez, Organometallics 2010, 29, 44914498.

[23] J. T. Sarmiento, S. Sua, A. Olmos, T. Varea, G. Asensio, ACS Catal. 2017, 7, 7146-7155.

[24] K. Fujita, Synlett 2016, 27, 1941-1944.

[25] C. Vriamont, M. Devillers, O. Riant, S. Hermans, Chem. Commun. 2013, 49, 10504-10506.

[26] R. Mihai, C. Rodríguez-Escrig, X. C. Cambeiro, E. C. Escudero-Adán, M. A. Pericàs, A. M. Echavarren, Chem. Commun. 2011, 47, 4893-4895.

[27] S. Tšupova, A. Cadu, S. A. C. Carabineiro, M. Rudolph, A. S. K. Hashmi, J. Catal. 2017, 350, 97-102.

[28] M. Egi, K. Azechi, S. Akai, Adv. Synth. Catal. 2011, 353, 287-290.

[29] W. Cao, B. Yu, Adv. Synth. Catal. 2011, 353, 1903-1907.

[30] M. A. N. Virboul, M. Lutz, M. A. Siegler, A. L. Spek, G. Van Koten, R. J. M. Klein Gebbink, Chem. - A Eur. J. 2009, 15, 9981-9986.

[31] M. R. Axet, O. Dechy-Cabaret, J. Durand, M. Gouygou, P. Serp, Coord. Chem. Rev. 2016, 308, 236-345.

[32] L. Xing, J. Xie, Y. Chen, L. Wang, Q. Zhou, Adv. Synth. Catal. 2008, 350, 10131016.

[33] J. D. Blakemore, A. Gupta, J. J. Warren, B. S. Brunschwig, H. B. Gray, J. Am. Chem. Soc. 2013, 135, 18288-18291.

[34] G. Liu, B. Wu, J. Zhang, X. Wang, M. Shao, J. Wang, Inorg. Chem. 2009, 48, 2383-2390.

[35] F. Li, B. Zhang, X. Li, Y. Jiang, L. Chen, Y. Li, L. Sun, Angew. Chemie - Int. Ed. 2011, 50, 12276-12279. 
[36] S. Sabater, J. A. Mata, E. Peris, ACS Catal. 2014, 4, 2038-2047.

[37] P. D. Tran, A. Le Goff, J. Heidkamp, B. Jousselme, N. Guillet, S. Palacin, H. Dau, M. Fontecave, V. Artero, Angew. Chemie - Int. Ed. 2011, 50, 1371-1374.

[38] Y. Suzuki, P. Laurino, D. T. Mcquade, P. H. Seeberger, Helv. Chim. Acta 2012, 95, 2578-2588.

[39] A. Mollar-Cuni, D. Ventura-Espinosa, S. Martín, Á. Mayoral, P. Borja, J. A. Mata, ACS Omega 2018, 5, 15217-15228.

[40] D. Didier, E. Schulz, Tetrahedron: Asymmetry 2013, 24, 769-775.

[41] L. Zhang, W. Zhang, P. Serp, W. H. Sun, J. Durand, ChemCatChem 2014, 6, 1310-1316.

[42] C. Vriamont, M. Devillers, O. Riant, S. Hermans, Chem. - A Eur. J. 2013, 19, 12009-12017.

[43] R. G. Nuzzo, D. L. Allara, J. Am. Chem. Soc. 1983, 13, 4481-4483.

[44] B. Zhang, J. Fang, J. Li, J. Lau, D. Mattia, Z. Zhong, J. Xie, N. Yan, Chem. - An Asian J. 2016, 11, 532-539.

[45] L. M. Martínez-Prieto, A. Ferry, L. Rakers, C. Richter, P. Lecante, K. Philippot, B. Chaudret, F. Glorius, Chem. Commun. 2016, 52, 4768-4771.

[46] L. M. Martínez-Prieto, L. Rakers, Á. M. López-Vinasco, I. Cano, Y. Coppel, K. Philippot, F. Glorius, B. Chaudret, P. W. N. M. Van Leeuwen, Chem. - A Eur. J. 2017, 23, 12779-12786.

[47] K. R. Brown, A. P. Fox, M. J. Natan, J. Am. Chem. Soc. 1996, 118, 1154-1157.

[48] L. O. Brown, J. E. Hutchison, J. Am. Chem. Soc. 1999, 121, 882-883.

[49] P. Maity, H. Tsunoyama, M. Yamauchi, S. Xie, T. Tsukuda, J. Am. Chem. Soc. 2011, 133, 20123-20125.

[50] W. W. Weare, S. M. Reed, M. G. Warner, J. E. Hutchison, J. Am. Chem. Soc. 2000, 122, 12890-12891.

[51] I. Cano, A. M. Chapman, A. Urakawa, P. W. N. M. Van Leeuwen, J. Am. Chem. Soc. 2014, 136, 2520-2528.

[52] L. Jin, D. S. Weinberger, M. Melaimi, C. E. Moore, A. L. Rheingold, G. Bertrand, Angew. Chemie - Int. Ed. 2014, 126, 9205-9209. 
[53] S. Roland, X. Ling, M. Pileni, Langmuir 2016, 32, 7683-7696.

[54] J. Vignolle, T. D. Tilley, Chem. Commun. 2009, 46, 7230-7232.

[55] J. Crespo, Y. Guari, A. Ibarra, J. Larionova, T. Lasanta, D. Laurencin, J. M. López-de-luzuriaga, M. Monge, M. E. Olmos, S. Richeter, Dalton Trans. 2014, 43, 15713-15718.

[56] Z. Cao, D. Kim, D. Hong, Y. Yu, J. Xu, S. Lin, X. Wen, E. M. Nichols, K. Jeong, J. A. Reimer, et al., J. Am. Chem. Soc. 2016, 138, 8120-8125.

[57] S. G. Song, C. Satheeshkumar, J. Park, J. Ahn, T. Premkumar, Y. Lee, C. Song, Macromolecules 2014, 47, 6566-6571.

[58] R. Ye, A. V Zhukhovitskiy, R. V Kazantsev, S. C. Fakra, B. B. Wickemeyer, F. D. Toste, G. A. Somorjai, J. Am. Chem. Soc. 2018, 140, 4144-4149.

[59] A. Collado, A. Gómez-Suárez, A. R. Martin, A. M. Z. Slawin, S. P. Nolan, Chem. Commun. 2013, 49, 5541-5543.

[60] D. Ventura-Espinosa, S. Sabater, A. Carretero-Cerdán, M. Baya, J. A. Mata, ACS Catal. 2018, 8, 2558-2566.

[61] M. Michalska, K. Grela, Synlett 2016, 27, 599-603.

[62] A. Zhdanko, M. E. Maier, Angew. Chemie - Int. Ed. 2014, 53, 7760-7764.

[63] C. A. Gaggioli, G. Ciancaleoni, L. Biasiolo, G. Bistoni, D. Zuccaccia, L. Belpassi, P. Belanzoni, F. Tarantelli, Chem. Commun. 2015, 51, 5990-5993.

[64] D. Ventura-Espinosa, C. Vicent, M. Baya, J. A. Mata, Catal. Sci. Technol. 2016, 6, 8024-8035.

[65] D. Ventura-Espinosa, A. Marzá-Beltrán, J. A. Mata, Chem. - A Eur. J. 2016, 22, 17758-17766.

[66] P. Ballestin, D. Ventura-Espinosa, S. Martín, A. Caballero, J. A. Mata, P. J. Pérez, Chem. - A Eur. J. 2019, 25, 9534-9539.

[67] L. Benhamou, V. César, H. Gornitzka, N. Lugan, G. Lavigne, Chem. Commun. 2009, 31, 4720-4722.

[68] M. Gatto, P. Belanzoni, L. Belpassi, L. Biasiolo, A. Del Zotto, F. Tarantelli, D. Zuccaccia, ACS Catal. 2016, 6, 7363-7376. 
[69] R. Torres-Mendieta, D. Ventura-Espinosa, S. Sabater, J. Lancis, G. MínguezVega, J. A. Mata, Sci. Rep. 2016, 6, 30478.

[70] H. Yin, H. Tang, D. Wang, Y. Gao, Z. Tang, ACS Nano 2012, 6, 8288-8297. 
CHAPTER 6

Experimental Section
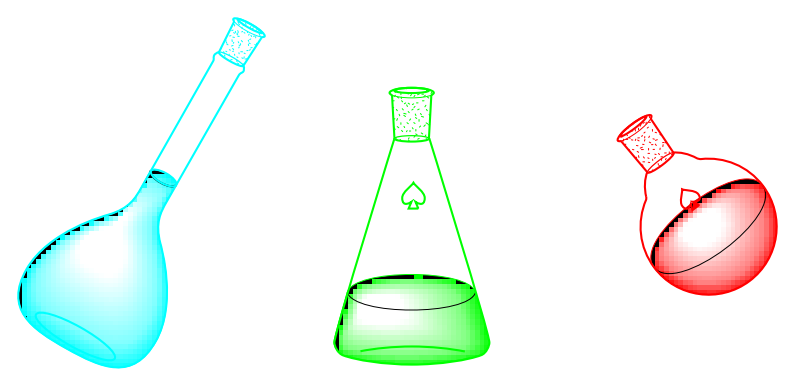



\subsection{ANALYTICAL TECHNIQUES Nuclear Magnetic Resonance (NMR)}

${ }^{1} \mathrm{H},{ }^{13} \mathrm{C}\left\{{ }^{1} \mathrm{H}\right\}$ and ${ }^{1} \mathrm{H}-{ }^{13} \mathrm{C}$ HSQC NMR spectra were recorded on the following spectrometers at $298 \mathrm{~K}$ :

Varian Innova $300 \mathrm{MHz}\left({ }^{1} \mathrm{H} 300 \mathrm{MHz},{ }^{13} \mathrm{C} 75 \mathrm{MHz}\right)$

Varian Innova $500 \mathrm{MHz}\left({ }^{1} \mathrm{H} 500 \mathrm{MHz},{ }^{13} \mathrm{C} 125 \mathrm{MHz}\right)$

Bruker Avance $300 \mathrm{MHz}\left({ }^{1} \mathrm{H} 300 \mathrm{MHz},{ }^{13} \mathrm{C} 75 \mathrm{MHz}\right)$

Bruker Avance $400 \mathrm{MHz}\left({ }^{1} \mathrm{H} 400 \mathrm{MHz},{ }^{13} \mathrm{C} 100 \mathrm{MHz}\right)$

Chemical shifts are given in ppm $(\delta)$, referred to the residual peak of the deuterated solvent $\left(\mathrm{CDCl}_{3}, \mathrm{CD}_{3} \mathrm{OD}\right.$ and DMSO- $\left.d_{6}\right)$ and reported downfield of $\mathrm{SiMe}_{4}$.

\section{Electrospray Mass Spectra (ESI-MS) and High Resolution Mass Spectroscopy (HRMS)}

Electrospray Mass Spectra (ESI-MS) were recorded on a Micromass Quatro LC instrument; $\mathrm{CH}_{3} \mathrm{OH}$ or $\mathrm{CH}_{3} \mathrm{CN}$ were used as mobile phase and nitrogen was employed as drying and nebulizing gas. High Resolution Mass Spectra (HRMS) were recorded on a QTOF I (quadrupole-hexapole-TOF) mass spectrometer with an orthogonal Z-spray-electrospray interface (Micromass, Manchester, UK). The drying gas as well as nebulizing gas was nitrogen at a flow of $400 \mathrm{~L} / \mathrm{h}$ and $80 \mathrm{~L} / \mathrm{h}$ respectively. The temperature of the source block was set to $120{ }^{\circ} \mathrm{C}$ and the desolvation temperature to $150^{\circ} \mathrm{C}$. A capillary voltage of $3.5 \mathrm{kV}$ was used in the positive scan mode and the cone voltage was set to $30 \mathrm{~V}$.

\section{Elemental Analysis (EA)}

Elemental analyses were carried out on a EuroEA3000 Eurovector Analyser.

\section{Gas Chromatography: GC and GC/MS}

GC analyses were obtained on a Shimadzu GC-2010 apparatus equipped with a FID and a Technokroma (TRB-5MS, $30 \mathrm{~m} \times 0.25 \mathrm{~mm} \times 0.25 \mu \mathrm{m}$ ) column and on a Shimadzu GCMS-QP2010 apparatus equipped with a Technokroma (TRB-5MS, $30 \mathrm{~m} \times$ $0.25 \mathrm{~mm} \times 0.25 \mu \mathrm{m}$ ) column. 


\section{UV/Vis Spectroscopy}

UV/Vis spectra were recorded between 250 and $600 \mathrm{~nm}$ by a Cary 300 Bio UV/Vis Varian spectrophotometer or using a Varian Cary 50 spectrophotometer. The samples were suspended in DMF and sonicated for $5 \mathrm{~min}$ before the measurements. Molecular complexes were dissolved in DMF $\left(10^{-6} \mathrm{M}\right)$.

\section{Scanning Electron Microscopy (SEM)}

Scanning Electron Micrographs of the samples were taken with a field emission gun scanning electron microscope (FEG-SEM) model JEOL 7001F, equipped with a spectrometer of energy dispersion of X-ray (EDS) from Oxford instruments. The samples for microstructural and microanalysis determinations were covered with a Pt film.

\section{High Resolution Transmission Electron Microscopy (HRTEM)}

High-Resolution images of Transmission Electron Microscopy (HRTEM) and High-Angle Annular Dark-Field (HAADF-STEM) images of the samples were obtained using a Jem-2100 LaB6 (JEOL) transmission electron microscope coupled with an INCA Energy TEM 200 (Oxford) Energy Dispersive X-ray spectrometer (EDS) operating at $200 \mathrm{kV}$. Samples were prepared by drying a droplet of a methanolic dispersion on a carbon-coated copper grid.

\section{X-Ray photoelectron spectroscopy (XPS)}

X-ray photoelectron spectra (XPS) were acquired on a Kratos AXIS ultra DLD spectrometer with a monochromatic Al K $\alpha$ X-ray source $(1486.6 \mathrm{eV})$ using a pass energy of $20 \mathrm{eV}$. To provide a precise energy calibration, the XPS binding energies were referenced to the $\mathrm{C} 1 \mathrm{~s}$ peak at $284.6 \mathrm{eV}$.

\subsection{SYNTHESIS AND CHARACTERISATION}

All manipulations were carried out by using standard Schlenk techniques under nitrogen atmosphere unless otherwise stated. Anhydrous solvents were dried using a solvent purification system (SPS M BRAUN) or purchased from Aldrich and degassed prior to use by purging with dry nitrogen and kept over molecular sieves. All other reagents were used as received from commercial suppliers. [AuCl( $\left.\left(\mathrm{SMe}_{2}\right)\right]^{[1]}$ 1-mesityl imidazole, ${ }^{[2]}$ 1-(bromomethyl)pyrene, ${ }^{[3]}$ 4-hydroxy-1,3-(2,6diisopropylphenyl)imidazolium chloride, ${ }^{[4]} 2$-(phenylethynyl) aniline, ${ }^{[5]}$ 
2-(n-butyl) aniline, ${ }^{[5]}$ ligand precursors: $\mathbf{A},{ }^{[6]} \mathbf{B},{ }^{[7]} \mathbf{C},{ }^{[8]} \mathbf{D},{ }^{[9]} \mathbf{E},{ }^{[10]} \mathbf{F}_{,}^{[11]} \mathbf{G}^{[12]}$ and metal complexes $\mathbf{3 C},{ }^{[8]} \mathbf{4 D},{ }^{[13]} \mathbf{7} \mathbf{G}^{[14]}$ and $\mathbf{9} \mathbf{C}^{[15]}$ were prepared as described in the literature.

\subsubsection{Synthesis and characterisation of ligand precursors Synthesis of $\mathrm{H}$}

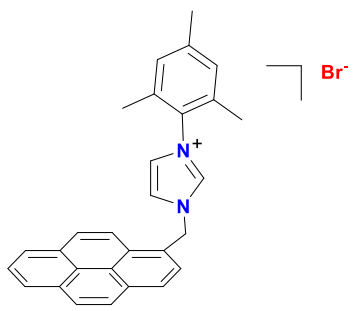

To a solution of 1-(bromomethyl)pyrene $(750 \mathrm{mg}$, $2.75 \mathrm{mmol})$ in dry THF ( $8 \mathrm{~mL}$ ) was added 1-mesityl imidazole (0.465 $\mathrm{mg}, 2.5 \mathrm{mmol})$. The mixture was refluxed under nitrogen for $16 \mathrm{~h}$. The resulting suspension was filtered off and washed with diethyl ether $(2 \times 10 \mathrm{~mL})$. The imidazolium salt $\mathbf{H}$ was obtained as an analytically pure white solid. Yield: $1.1 \mathrm{~g}(90 \%) .{ }^{1} \mathrm{H}$ NMR (400 MHz, DMSO): $\delta 9.60$ (s, 1H, $\mathrm{NCHN}), 8.47-8.14\left(\mathrm{~m}, 9 \mathrm{H}, \mathrm{CH}_{\text {pyr }}\right), 8.06\left(\mathrm{~s}, 1 \mathrm{H}, \mathrm{CH}_{\text {imid }}\right), 7.95\left(\mathrm{~s}, 1 \mathrm{H}, \mathrm{CH}_{\text {imid }}\right), 7.11(\mathrm{~s}, 2 \mathrm{H}$, $\left.\mathrm{CH}_{\text {mes }}\right), 6.34\left(\mathrm{~s}, 2 \mathrm{H}, \mathrm{N}-\mathrm{CH}_{2}\right), 2.31\left(\mathrm{~s}, 3 \mathrm{H}, \mathrm{CH}_{3, \text { mes }}\right), 1.97\left(\mathrm{~s}, 6 \mathrm{H}, \mathrm{CH}_{3, \text { mes }}\right) .{ }^{13} \mathrm{C}\left\{{ }^{1} \mathrm{H}\right\} \mathrm{NMR}$ (101 MHz, DMSO) $140.7\left(C_{\text {pyr }}\right), 138.2(\mathrm{NCHN}), 134.7-125.7\left(\mathrm{CH}_{\mathrm{pyr}}, C_{\mathrm{pyr}}, \mathrm{CH}_{\mathrm{mes}}, C_{\mathrm{mes}}\right)$, $124.8\left(\mathrm{CH}_{\text {imid }}\right), 124.7-124.2\left(C_{\text {pyr }}, C_{\text {mes }}\right), 123.8\left(\mathrm{CH}_{\text {imid }}\right), 122.7\left(\mathrm{CH}_{\text {pyr }}\right), 51.2\left(\mathrm{CH}_{2}\right), 21.0$ $\left(\mathrm{CH}_{3, \text { mes }}\right), 17.3\left(\mathrm{CH}_{3}\right.$,mes $)$. Anal. Calcd. for $\mathrm{C}_{29} \mathrm{H}_{25} \mathrm{~N}_{2} \mathrm{Br}(481.42 \mathrm{~g} / \mathrm{mol}): \mathrm{C}, 72.34 ; \mathrm{H}, 5.23$; $\mathrm{N}, 5.81$. Found: C, 71.89; H, 5.51; N, 6.09. Electrospray MS (Cone $20 \mathrm{~V})(\mathrm{m} / \mathrm{z}$, fragment): $401.3[\mathrm{M}]^{+}$

\section{Synthesis of I}

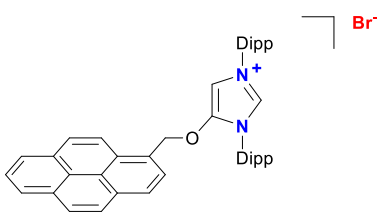

4-hydroxy-1,3-(2,6-diisopropylphenyl)imidazolium chloride (600 mg, $1.36 \mathrm{mmol}$ ) was suspended in $30 \mathrm{~mL}$ of dry acetonitrile. Potassium carbonate $(234 \mathrm{mg}, 1.7 \mathrm{mmol}$ ) and potassium bromide (490 $\mathrm{mg}, 4.08 \mathrm{mmol}$ ) were added and the mixture stirred at $0{ }^{\circ} \mathrm{C}$ for $1 \mathrm{~h}$. Then, 1-(bromomethyl)pyrene (482 $\mathrm{mg}, 1,63 \mathrm{mmol}$ ) was added and the mixture stirred for $2 \mathrm{~h}$ at room temperature and heated at $60^{\circ} \mathrm{C}$ for $15 \mathrm{~h}$. The solvent was removed and the residue was suspended in dichloromethane and was filtered to remove insoluble inorganic salts. Recrystallization from dichloromethane/ether at $-18{ }^{\circ} \mathrm{C}$ afforded I as a pale yellow powder. Yield: $330 \mathrm{mg}(42 \%) .{ }^{1} \mathrm{H} \mathrm{NMR}\left(300 \mathrm{MHz}, \mathrm{CD}_{3} \mathrm{OD}\right) \delta 9.53\left(\mathrm{~d},{ }^{4} J_{\mathrm{H}-}\right.$ $\mathrm{H}=1.8 \mathrm{~Hz}, 1 \mathrm{H}, \mathrm{NCHN}), 8.46-8.02\left(\mathrm{~m}, 9 \mathrm{H}, \mathrm{CH}_{\text {pyr }}\right), 7.97\left(\mathrm{~d},{ }^{4} J_{\mathrm{H}-\mathrm{H}}=1.8 \mathrm{~Hz}, 1 \mathrm{H}, \mathrm{CH}_{\text {imid }}\right)$, $7.64\left(\mathrm{~m}, 1 \mathrm{H}, \mathrm{CH}_{\text {arom }}\right), 7.49\left(\mathrm{~m}, 3 \mathrm{H}, \mathrm{CH}_{\text {arom }}\right), 7.32\left(\mathrm{~m}, 2 \mathrm{H}, \mathrm{CH}_{\text {arom }}\right), 6.09\left(\mathrm{~s}, 2 \mathrm{H}, \mathrm{CH}_{2}\right), 2.72$ $-2.44\left(\mathrm{~m}, 2 \mathrm{H}, \mathrm{CH}_{\mathrm{iPr}}\right), 2.43-2.18\left(\mathrm{~m}, 2 \mathrm{H}, \mathrm{CH}_{\mathrm{iPr}}\right), 1.32\left(\mathrm{~d},{ }^{3} J_{\mathrm{H}-\mathrm{H}}=6.8 \mathrm{~Hz}, 6 \mathrm{H}, \mathrm{CH}_{3, \mathrm{Pr}}\right), 1.21$ $\left(\mathrm{d},{ }^{3} J_{\mathrm{H}-\mathrm{H}}=6.9 \mathrm{~Hz}, 6 \mathrm{H}, \mathrm{CH}_{3, \mathrm{iPr}}\right), 1.10\left(\mathrm{~d},{ }^{3} J_{\mathrm{H}-\mathrm{H}}=6.9 \mathrm{~Hz}, 6 \mathrm{H}, \mathrm{CH}_{3, \mathrm{iPr}}\right), 0.95\left(\mathrm{~d},{ }^{3} J_{\mathrm{H}-\mathrm{H}}=6.8 \mathrm{~Hz}\right.$, $\left.6 \mathrm{H}, \mathrm{CH}_{3, \mathrm{ipr}}\right) .{ }^{13} \mathrm{C}\left\{{ }^{1} \mathrm{H}\right\}$ NMR $\left(75 \mathrm{MHz}, \mathrm{CD}_{3} \mathrm{OD}\right) \delta[149.4,147.1,146.7,135.1,134.1]\left(C_{\text {pyr }}\right.$, 
$C_{\text {arom) }}, 133.4(\mathrm{NCHN}),[132.6,132.1,132.0,131.3,129.9,129.7,129.6,128.3,127.8$, $127.6,127.6,127.2,127.0,126.1,126.0,125.8,125.5,123.6]\left(C_{\text {pyr }}, C_{\text {arom }}, C_{\text {imid }}\right), 105.4$ $\left(\mathrm{CH}_{\text {imid }}\right), 74.9\left(\mathrm{CH}_{2}\right),[30.5,30.3]\left(\mathrm{CH}_{\mathrm{iPr}}\right),[24.9,24.7,24.0,23.2]\left(\mathrm{CH}_{3, \mathrm{iPr}}\right)$. Anal. Calcd. for $\mathrm{C}_{44} \mathrm{H}_{47} \mathrm{~N}_{2} \mathrm{OBr}(699.76 \mathrm{~g} / \mathrm{mol}): \mathrm{C}, 75.52 ; \mathrm{H}, 6.77 ; \mathrm{N}, 4.00$ Found: $\mathrm{C}, 75.38 ; \mathrm{H}, 6.74 ; \mathrm{N}$, 3.85. Electrospray MS (Cone $20 \mathrm{~V})$ (m/z, fragment): $619.4[\mathrm{M}]^{+}$.

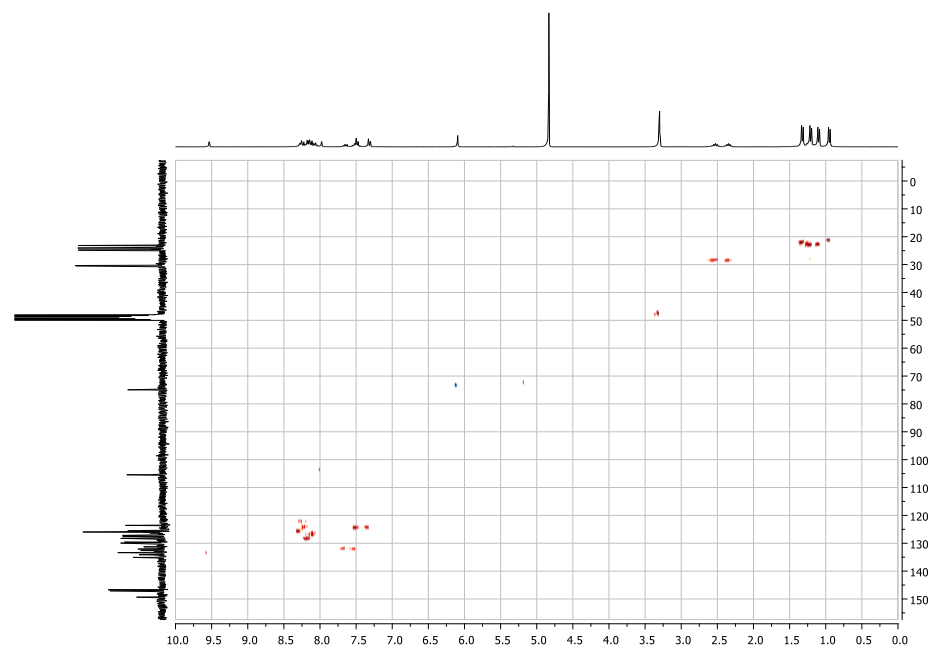

${ }^{1} \mathrm{H}-{ }^{13} \mathrm{C} \mathrm{HSQC}$ NMR spectrum $\left({ }^{1} \mathrm{H}, 300 \mathrm{MHz}\right.$ and $\left.{ }^{13} \mathrm{C}, 75 \mathrm{MHz}\right)$ of I in MeOD

\subsubsection{Synthesis and characterisation of metal complexes}

\section{Synthesis of $1 \mathrm{~A}$}

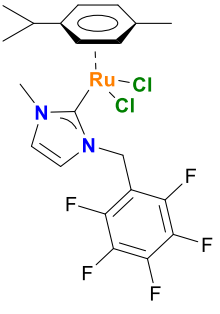

Silver oxide (102 $\mathrm{mg}, 0.44 \mathrm{mmol}$ ) was added to a solution of 1 - pentafluorobenzyl - 3 - methylimidazolium bromide $(248 \mathrm{mg}$, $0.72 \mathrm{mmol}$ ) in $\mathrm{CH}_{2} \mathrm{Cl}_{2}$ in a round bottom flask covered with aluminium foil. The suspension was stirred at room temperature for $1 \mathrm{~h}$. Then $\left[\mathrm{Ru}(p-c y m) \mathrm{Cl}_{2}\right]_{2}(200 \mathrm{mg}, 0.32 \mathrm{mmol})$ was added to the suspension and stirred overnight at reflux temperature. After solvent removal, the crude product was purified by column chromatography. The pure compound was eluted with dichloromethane/acetone (9:1). After solvent removal, compound $\mathbf{1 A}$ was obtained as an orange solid. Yield: $120 \mathrm{mg}(33 \%) .{ }^{1} \mathrm{H}$ NMR (500 MHz, $\mathrm{CDCl}_{3}$ ): $\delta 6.99$ (s, $\left.1 \mathrm{H}, \mathrm{CH}_{\text {imid }}\right), 6.61\left(\mathrm{~s}, 1 \mathrm{H}, \mathrm{CH}_{\text {imid }}\right)$, $5.65\left(\mathrm{~s}, 2 \mathrm{H}, \mathrm{NCH}_{2}\right), 5.51\left(\mathrm{~s}, 2 \mathrm{H}, \mathrm{CH}_{p \text {-cym }}\right), 5.21\left(\mathrm{~s}, 2 \mathrm{H}, \mathrm{CH}_{p \text {-cym }}\right), 4.00$ (s, 3H, $\left.\mathrm{NCH}_{3}\right), 2.96$ $\left(\mathrm{m}, 1 \mathrm{H}, \mathrm{CH}_{\text {iPr }}\right), 2.07\left(\mathrm{~s}, 3 \mathrm{H}, \mathrm{CH}_{3, p-c y m}\right) 1.27\left(\mathrm{~d},{ }^{3} J_{\mathrm{H}-\mathrm{H}}=6.9 \mathrm{~Hz}, 6 \mathrm{H}, \mathrm{CH}_{3, i \mathrm{Pr}}\right) .{ }^{13} \mathrm{C}\left\{{ }^{1} \mathrm{H}\right\} \mathrm{NMR}$ $\left(125 \mathrm{MHz}, \mathrm{CDCl}_{3}\right): \delta 175.9\left(C_{\text {carbene }}-\mathrm{Ru}\right),[146.8,144.8,142.7,140.9,139.0,136.9]$ $(C-F),[124.5,120.7]\left(C_{\text {imid }}\right),[109.0,99.1]\left(C_{p-c y m}\right),[85.7,81.9]\left(C_{p-c y m}\right), 44.3\left(\mathrm{~N}-\mathrm{CH}_{2}\right)$, 
$40.0\left(\mathrm{NCH}_{3}\right), \quad 31.0\left(\mathrm{CH}_{i \mathrm{Pr}}\right), 22.3\left(\mathrm{CH}_{3, p-c y m}\right), 18.8\left(\mathrm{CH}_{3, i \mathrm{Pr}}\right)$. Anal. Calcd. for $\mathrm{C}_{21} \mathrm{H}_{21} \mathrm{~N}_{2} \mathrm{~F}_{5} \mathrm{RuCl}_{2}(568.37 \mathrm{~g} / \mathrm{mol}): \mathrm{C}, 44.37 ; \mathrm{H}, 3.72 ; \mathrm{N}, 4.92$. Found: $\mathrm{C}, 44.49 ; \mathrm{H}, 3.56$; $\mathrm{N}, 4.89$. HRMS ESI-TOF-MS (positive mode): [M-Cl] ${ }^{+}$monoisotopic peak 533.0357; calc 533.0359, er: 0.4 ppm.

\section{Synthesis of 2B}

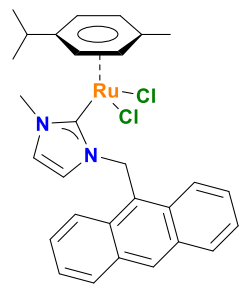

Silver oxide (102 $\mathrm{mg}, 0.44 \mathrm{mmol}$ ) was added to a solution of 1 - (9 - methylantracene) - 3- methylimidazolium chloride (223 mg, $0.72 \mathrm{mmol}$ ) in $\mathrm{CH}_{2} \mathrm{Cl}_{2}$ in a round bottom flask covered with aluminium foil. The suspension was stirred at room temperature for $1 \mathrm{~h}$. Then $\left[\mathrm{Ru}(p-\mathrm{cym}) \mathrm{Cl}_{2}\right]_{2}(200 \mathrm{mg}, 0.32 \mathrm{mmol})$ was added to the suspension and stirred overnight at reflux temperature. After solvent removal, the crude product was purified by column chromatography. The pure compound was eluted with dichloromethane/acetone (95:5). Recrystallization from a dichloromethane/hexane mixture afforded compound $2 \mathrm{~B}$ as an orange solid. Yield: $170 \mathrm{mg}$ (46 \%). ${ }^{1} \mathrm{H}$ NMR (500 MHz, $\mathrm{CDCl}_{3}$ ): $\delta 8.52$ (s, $1 \mathrm{H}, \mathrm{CH}_{\text {ant }}$ ), 8.26 (d, $\left.{ }^{3} J_{\mathrm{H}-\mathrm{H}}=8.7 \mathrm{~Hz}, 2 \mathrm{H}, \mathrm{CH}_{\text {ant }}\right), 8.02\left(\mathrm{~d},{ }^{3} \mathrm{~J}_{\mathrm{H}-\mathrm{H}}=8.2 \mathrm{~Hz}, 2 \mathrm{H}, \mathrm{CH}_{\text {ant }}\right), 7,56-7,40\left(\mathrm{~m}, 4 \mathrm{H}, \mathrm{CH}_{\mathrm{ant}}\right)$, $6.64\left(\mathrm{~d}, 3^{3} \mathrm{H}_{\mathrm{H}-\mathrm{H}}=2.0 \mathrm{~Hz}, 1 \mathrm{H}, \mathrm{CH}_{\text {imid }}\right), 6.00\left(\mathrm{~d},{ }^{3} J_{\mathrm{H}-\mathrm{H}}=1.9 \mathrm{~Hz}, 1 \mathrm{H}, \mathrm{CH}_{\text {imid }}\right), 5.58(\mathrm{~s}, 2 \mathrm{H}$, $\left.\mathrm{CH}_{\text {p-cym }}\right), 5.36\left(\mathrm{~s}, 2 \mathrm{H}, \mathrm{CH}_{\text {p-cym }}\right), 4.02\left(\mathrm{~s}, 3 \mathrm{H}, \mathrm{NCH}_{3}\right), 3.02\left(\mathrm{~m}, 1 \mathrm{H}, \mathrm{CH}_{\text {ipr, }}\right), 2.24(\mathrm{~s}, 3 \mathrm{H}$, $\left.\mathrm{CH}_{3, p-\mathrm{cym}}\right), 1.31\left(\mathrm{~d},{ }^{3} \mathrm{~J}_{\mathrm{H}-\mathrm{H}}=6.9 \mathrm{~Hz}, 6 \mathrm{H}, \mathrm{CH}_{3, \text { ipr }}\right) .{ }^{13} \mathrm{C}\left\{{ }^{1} \mathrm{H}\right\} \mathrm{NMR}\left(125 \mathrm{MHz}, \mathrm{CDCl}_{3}\right): \delta 172.6$ $\left(C_{\text {carbene }}-\mathrm{Ru}\right),[131.7,131.4,129.3,129.1,127.3,125.4,125.3,124.2,122.7,121.7]$ $\left(C_{\text {ant }}, \mathrm{CH}_{\text {imid }}\right),[108.3,99.5]\left(C_{p-c y m}\right),[84.8,82.9]\left(\mathrm{CH}_{p-\text { cym }}\right), 47.7\left(\mathrm{~N}^{-\mathrm{CH}_{2}}\right), 39.9\left(\mathrm{NCH}_{3}\right)$, $31.1\left(\mathrm{CH}_{\text {ipr }}\right), 23.0\left(\mathrm{CH}_{3}, \quad\right.$ p-cym $), 19.0\left(\mathrm{CH}_{3, i \mathrm{ir}}\right)$. Anal. Calcd. for $\mathrm{C}_{29} \mathrm{H}_{30} \mathrm{~N}_{2} \mathrm{RuCl}_{2}$ $(578.54 \mathrm{~g} / \mathrm{mol}): \mathrm{C}, 60.20 ; \mathrm{H}, 5.22 ; \mathrm{N}, 4.84$. Found: $\mathrm{C}, 60.49 ; \mathrm{H}, 5.02 ; \mathrm{N}, 4.89$ ESI-TOF-MS (positive mode): [M-Cl] ${ }^{+}$monoisotopic peak 543.1140; calc 543.1146, Er: $1.6 \mathrm{ppm}$.

\section{Synthesis of $5 E$}

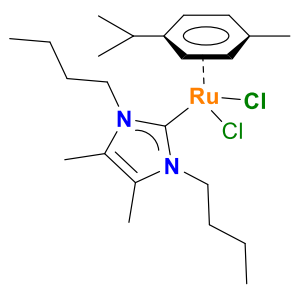

Silver oxide (126 mg, $0.54 \mathrm{mmol}$ ) was added to a solution of 4,5-dimethyl-1,3-bis(butyl)imidazolium chloride (178 mg, $0.72 \mathrm{mmol}$ ) in $\mathrm{CH}_{2} \mathrm{Cl}_{2}$. The solution was stirred at room temperature overnight and filtered through celite. Then, $\left[\mathrm{Ru}(p-\mathrm{cym}) \mathrm{Cl}_{2}\right]_{2}(200 \mathrm{mg}, 0.32 \mathrm{mmol})$ was added to the solution and stirred at room temperature overnight. After solvent removal, the crude product was purified by column chromatography. The pure compound 5E was eluted with dichloromethane/acetone (9:1) and precipitated in a 
mixture of dichloromethane/hexane to give an orange solid. Yield: $44 \mathrm{mg}$ (13.1\%). ${ }^{1} \mathrm{H}$ NMR $\left(500 \mathrm{MHz}, \mathrm{CDCl}_{3}\right) \delta 5.37\left(\mathrm{~d},{ }^{3} \mathrm{~J}_{\mathrm{H}-\mathrm{H}}=5.9 \mathrm{~Hz}, 2 \mathrm{H}, \mathrm{CH}_{p-\mathrm{cym}}\right), 5.00\left(\mathrm{~d},{ }^{3} \mathrm{~J}_{\mathrm{H}-\mathrm{H}}=5.9 \mathrm{~Hz}\right.$, $\left.2 \mathrm{H}, \mathrm{CH}_{\text {p-cym}}\right), 4.40\left(\mathrm{~m}, 2 \mathrm{H}, \mathrm{CH}_{2, n-\mathrm{Bu}}\right), 3.98\left(\mathrm{~m}, 2 \mathrm{H}, \mathrm{CH}_{2, n-\mathrm{Bu}}\right), 2.93\left(\mathrm{~m}, 1 \mathrm{H}, \mathrm{CH}_{\mathrm{ipr}}\right), 2.17(\mathrm{~s}$, $\left.6 \mathrm{H}, \mathrm{CH}_{3, \text { imid }}\right), 1.96\left(\mathrm{~s}, 3 \mathrm{H}, \mathrm{CH}_{3, p-c y m}\right), 1.96\left(\mathrm{~m}, 2 \mathrm{H}, \mathrm{CH}_{2, n-\mathrm{Bu}}\right), 1.48\left(\mathrm{~m}, 4 \mathrm{H}, \mathrm{CH}_{2, n-\mathrm{Bu}}\right), 1.31$ $\left(\mathrm{m}, 2 \mathrm{H}, \mathrm{CH}_{2, n-\mathrm{Bu}}\right), 1.26\left(\mathrm{~d},{ }^{3} J_{\mathrm{H}-\mathrm{H}}=7.0 \mathrm{~Hz}, 6 \mathrm{H}, \mathrm{CH}_{3, \mathrm{Pr}}\right), 0.93\left(\mathrm{t},{ }^{3} J_{\mathrm{H}-\mathrm{H}}=7.1 \mathrm{~Hz}, 6 \mathrm{H}, \mathrm{CH}_{3, n-\mathrm{Bu}}\right)$. ${ }^{13} \mathrm{C}\left\{{ }^{1} \mathrm{H}\right\} \operatorname{NMR}\left(75 \mathrm{MHz}, \mathrm{CDCl}_{3}\right): \delta 169.9\left(C_{\text {carbene }}\right), 126.9\left(C_{\text {imid }}\right),[107.9,98.7]\left(\mathrm{C}_{\text {p-cym }}\right)$, [85.9, 85.9, 82.3, 82.3] $\left(\mathrm{CH}_{\text {p-cym }}\right), 49.8\left(\mathrm{CH}_{2, n-\mathrm{Bu}}\right), 33.9\left(\mathrm{CH}_{2, n-\mathrm{Bu}}\right), 30.7\left(\mathrm{CH}_{\text {iPr, }}\right), 22.7$ $\left(\mathrm{CH}_{3, \text { iPr }}\right), 20.3\left(\mathrm{CH}_{2, n-\mathrm{Bu}}\right), 18.6\left(\mathrm{CH}_{3, p-c y m}\right), 14.0\left(\mathrm{CH}_{3, n-\mathrm{Bu}}\right), 9.8\left(\mathrm{CH}_{3, \text { imid }}\right)$. Anal. Calcd. for $\mathrm{C}_{23} \mathrm{H}_{38} \mathrm{~N}_{2} \mathrm{RuCl}_{2}$ (514.54 g/mol): C, 53.68; H, 7.44; N, 5.44. Found: C, 54.0; H, 7.07; N, 5.24. Electrospray MS (cone $20 \mathrm{~V} ; \mathrm{m} / \mathrm{z}$, fragment): 479.3 [M-Cl] $]^{+}$HRMS ESI-TOF-MS (positive mode): [M-Cl] $]^{+}$monoisotopic peak 479.1770; calc. 479.1770, عr 0 ppm.

\section{Synthesis of $6 \mathrm{~F}$}

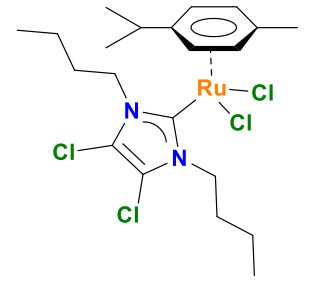

The reaction was carried out following the same procedure described for $\mathbf{5 E}$, with 4,5-dichloro-1,3-bis(butyl)imidazolium iodide (138 $\mathrm{mg}, 0.37 \mathrm{mmol}$ ), and $\mathrm{Ag}_{2} \mathrm{O}$ (69mg, $0.28 \mathrm{mmol}$ ) in $\mathrm{CH}_{2} \mathrm{Cl}_{2}$, and subsequently reacted with $\left[\mathrm{Ru}(p-c y m) \mathrm{Cl}_{2}\right]_{2}(100 \mathrm{mg}$, $0.17 \mathrm{mmol}$ ) to afford $6 \mathrm{~F}$ as an orange solid. Yield: $67 \mathrm{mg}$ (37.7 \%). ${ }^{1} \mathrm{H}$ NMR $\left(300 \mathrm{MHz}, \mathrm{CDCl}_{3}\right): \delta 5.45\left(\mathrm{~d},{ }^{3} \mathrm{~J}_{\mathrm{H}-\mathrm{H}}=6.0 \mathrm{~Hz}, 2 \mathrm{H}\right.$, $\left.\mathrm{CH}_{p \text {-cym }}\right), 5.08\left(\mathrm{~d},{ }^{3} \mathrm{~J}_{\mathrm{H}-\mathrm{H}}=6.0 \mathrm{~Hz}, 2 \mathrm{H}, \mathrm{CH}_{p-\text { cym }}\right), 4.53(\mathrm{~m}, 2 \mathrm{H}$, $\left.\mathrm{CH}_{2, n-\mathrm{Bu}}\right), 4.19\left(\mathrm{~m}, 2 \mathrm{H}, \mathrm{CH}_{2, n-\mathrm{Bu}}\right), 2.95\left(\mathrm{~m}, 1 \mathrm{H}, \mathrm{CH}_{\text {ipr, } p \text {-cym }}\right), 2.02\left(\mathrm{~s}, 3 \mathrm{H}, \mathrm{CH}_{3, p-\mathrm{cym}}\right), 2.02(\mathrm{~m}$, $\left.2 \mathrm{H}, \mathrm{CH}_{2, n-\mathrm{Bu}}\right) 1,74\left(\mathrm{~m}, 2 \mathrm{H}, \mathrm{CH}_{2, n-\mathrm{Bu}}\right), 1.41\left(\mathrm{~m}, 4 \mathrm{H}, \mathrm{CH}_{2, n-\mathrm{Bu}}\right), 1.29\left(\mathrm{~d},{ }^{3} \mathrm{~J}_{\mathrm{H}-\mathrm{H}}=6.9 \mathrm{~Hz}, 6 \mathrm{H}\right.$, $\left.\mathrm{CH}_{3, \mathrm{Pr}}\right), 0.96\left(\mathrm{t},{ }^{3} \mathrm{~J}_{\mathrm{H}-\mathrm{H}}=7.3 \mathrm{~Hz}, 6 \mathrm{H}, \mathrm{CH}_{3, n-\mathrm{Bu}}\right) .{ }^{13} \mathrm{C}\left\{{ }^{1} \mathrm{H}\right\} \mathrm{NMR}\left(75 \mathrm{MHz}, \mathrm{CDCl}_{3}\right): \delta 175.2$ $\left(\mathrm{C}_{\text {carbene }}\right), 117.8\left(\mathrm{C}_{\text {imid }}\right),[108.4,98.9]\left(\mathrm{C}_{p \text {-cym }}\right),[86.4,82.8]\left(\mathrm{C}_{p-\text { cym }}\right), 51.4\left(\mathrm{CH}_{2, n-\mathrm{Bu}}\right)$, $33.2\left(\mathrm{CH}_{2, n-\mathrm{Bu}}\right), 30.6\left(\mathrm{CH}_{\text {pPr }}\right), 22.5\left(\mathrm{CH}_{3, i \mathrm{Pr}}\right), 20.0\left(\mathrm{CH}_{2, n-\mathrm{Bu}}\right), 18.7\left(\mathrm{CH}_{3, p-\mathrm{cym}}\right), 13.7\left(\mathrm{CH}_{3, n-\mathrm{Bu}}\right)$. Anal. Calcd. for $\mathrm{C}_{21} \mathrm{H}_{32} \mathrm{~N}_{2} \mathrm{RuCl}_{4}(555.38 \mathrm{~g} / \mathrm{mol}): \mathrm{C}, 45.41 ; \mathrm{H}, 5.80 ; \mathrm{N}, 5.04$. Found: $\mathrm{C}, 45.91 ; \mathrm{H}, 5.80 ; \mathrm{N}, 4.90$. Electrospray MS (cone $20 \mathrm{~V} ; \mathrm{m} / \mathrm{z}$, fragment): 519.1 [M-Cl] $^{+}$. HRMS ESI-TOF-MS (positive mode): $[\mathrm{M}-\mathrm{Cl}]^{+}$monoisotopic peak 519.0671; calc. 519.0672, er 0.2 ppm. 


\section{Synthesis of 7G'}

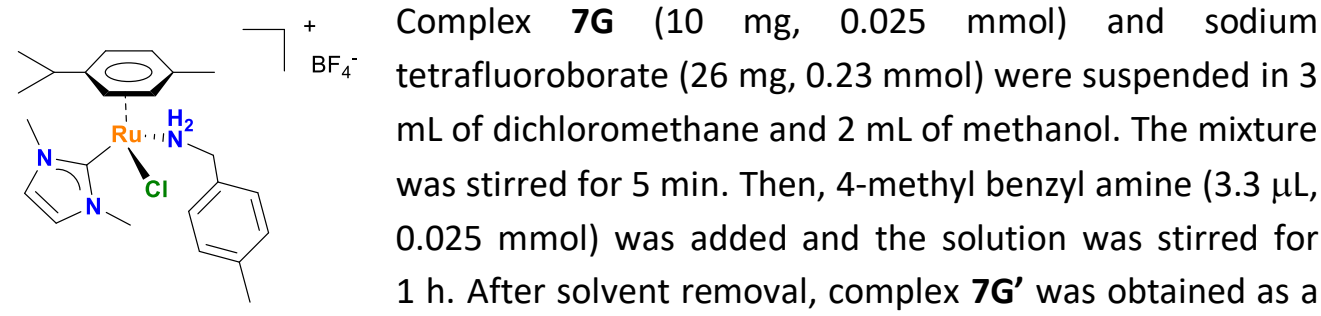
pale yellow solid. ${ }^{1} \mathrm{H} \mathrm{NMR}\left(500 \mathrm{MHz}, \mathrm{CDCl}_{3}\right) \delta 7.35\left(\mathrm{~d},{ }^{3} \mathrm{~J}_{\mathrm{H}-\mathrm{H}}=7.9 \mathrm{~Hz}, 2 \mathrm{H}, \mathrm{CH}_{\text {amine }}\right), 7.17$ - $6.99\left(\mathrm{~m}, 4 \mathrm{H}, \mathrm{CH}_{\text {amine; }} \mathrm{CH}_{\text {imid }}\right), 5.81\left(\mathrm{~d},{ }^{3} \mathrm{~J}_{\mathrm{H}-\mathrm{H}}=6.0 \mathrm{~Hz}, 1 \mathrm{H}, \mathrm{CH}_{\text {p-cym }}\right), 5.76(\mathrm{~d}$, $\left.{ }^{3} J_{\mathrm{H}-\mathrm{H}}=6.0 \mathrm{~Hz}, 1 \mathrm{H}, \mathrm{CH}_{p-\text { cym }}\right), 5.68\left(\mathrm{~d},{ }^{3} \mathrm{~J}_{\mathrm{H}-\mathrm{H}}=6.0 \mathrm{~Hz}, 1 \mathrm{H}, \mathrm{CH}_{p-\mathrm{cym}}\right), 5.45\left(\mathrm{~d},{ }^{3} \mathrm{~J}_{\mathrm{H}-\mathrm{H}}=6.0 \mathrm{~Hz}\right.$, $\left.1 \mathrm{H}, \mathrm{CH}_{p \text {-cym }}\right), 4.09\left(\mathrm{~m}, 2 \mathrm{H}, \mathrm{CH}_{2}\right), 3.99\left(\mathrm{~s}, 3 \mathrm{H}, \mathrm{NCH}_{3}\right), 3.75\left(\mathrm{~s}, 3 \mathrm{H}, \mathrm{NCH}_{3}\right), 2.84(\mathrm{~m}, 1 \mathrm{H}$,

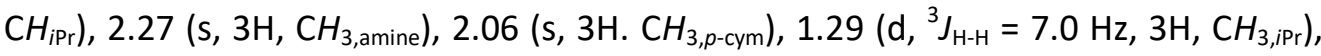
$1.09\left(\mathrm{~d},{ }^{3} \mathrm{~J}_{\mathrm{H}-\mathrm{H}}=6.8 \mathrm{~Hz}, 3 \mathrm{H}, \mathrm{CH}_{3, \mathrm{PP}}\right) .{ }^{13} \mathrm{C} \mathrm{NMR}\left(126 \mathrm{MHz} \mathrm{CDCl}_{3}\right) \delta 172.6\left(C_{\text {carbene }}\right)$, [138.0, 136.6, 129.8, 129.1] ( $\left.C_{\text {amine }}\right),[125.1,124.3]\left(C_{\text {imid }}\right),[112.2,100.0]\left(C_{p-c y m}\right),[85.4,84.2$, 83.4, 82.8] $\left(\mathrm{CH}_{p-c y m}\right), 53.6\left(\mathrm{CH}_{2}\right),[39.7,38.4]\left(\mathrm{N}-\mathrm{CH}_{3}\right), 31.1\left(\mathrm{CH}_{\text {PPr }}\right),[24.0,21.3]$ $\left(\mathrm{CH}_{3, p-c y m}\right)$, [20.9, 18.4] $\left(\mathrm{CH}_{3, \mathrm{Pr}}\right)$. HRMS ESI-TOF-MS (positive mode): [M - $\left.\mathrm{BF}_{4}\right]^{+}$ monoisotopic peak 488.1415; calc. 488.1410, $\varepsilon_{\mathrm{r}}$ : 1.0 ppm.

\section{Synthesis of $8 \mathrm{C}$}

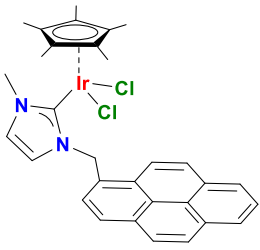

In a Schlenk, a mixture of imidazolium salt C (95 $\mathrm{mg}, 0.25 \mathrm{mmol})$ and potassium tert-butoxide ( $33 \mathrm{mg}, 0.280 \mathrm{mmol}$ ) was cooled to $0{ }^{\circ} \mathrm{C}$, in an ice bath. Freshly distilled tetrahydrofuran $(5 \mathrm{~mL})$ was added, and the mixture was stirred for $10 \mathrm{~min}$ and allowed to reach room temperature. Then, $\left[\mathrm{Cp}^{*} \mathrm{IrCl}_{2}\right]_{2}(100 \mathrm{mg}, 0.125 \mathrm{mmol})$ and $\mathrm{KCl}(243 \mathrm{mg}, 3.25 \mathrm{mmol}$ ) were added and the reaction mixture was stirred for $4 \mathrm{~h}$ at room temperature. The resulting suspension was filtered through celite and the solvent was removed under reduced pressure. The crude solid was purified by column chromatography. An elution with a mixture of dichloromethane and acetone (9:1) produced the separation of a yellow band containing the desired product. Precipitation from dichloromethane/hexane produced $8 \mathrm{C}$ as an analytically pure yellow solid. Yield: $111 \mathrm{mg}(64 \%) .{ }^{1} \mathrm{H}$ NMR $\left(300 \mathrm{MHz}, \mathrm{CDCl}_{3}\right): \delta 8.37\left(\mathrm{~d},{ }^{3} \mathrm{~J}_{\mathrm{H}-\mathrm{H}}=9.2 \mathrm{~Hz}, 1 \mathrm{H}, \mathrm{CH}_{\text {pyr }}\right), 8.25-8.02\left(\mathrm{~m}, 7 \mathrm{H}, \mathrm{CH}_{\text {pyr }}\right), 7.84$ $\left(\mathrm{d},{ }^{3} J_{\mathrm{H}-\mathrm{H}}=7.8 \mathrm{~Hz}, 1 \mathrm{H}, \mathrm{CH}_{\text {pyr }}\right), 6.90\left(\mathrm{~d},{ }^{3} \mathrm{~J}_{\mathrm{H}-\mathrm{H}}=2.1 \mathrm{~Hz}, 1 \mathrm{H}, \mathrm{CH}_{\text {imid }}\right), 6.66,6.28(\mathrm{AB}$, $\left.{ }^{2} J_{A B}=15.1 \mathrm{~Hz}, 2 \mathrm{H}, \mathrm{CH}_{2}\right), 6.65\left(\mathrm{~d},{ }^{3} \mathrm{~J}_{\mathrm{H}-\mathrm{H}}=2.1 \mathrm{~Hz}, 1 \mathrm{H}, \mathrm{CH}_{\text {imid }}\right), 4.07\left(\mathrm{~s}, 3 \mathrm{H}, \mathrm{NCH}_{3}\right), 1.62(\mathrm{~s}$, $\left.15 \mathrm{H}, \mathrm{C}\left(\mathrm{CH}_{3}\right)_{5}\right) .{ }^{13} \mathrm{C}\left\{{ }^{1} \mathrm{H}\right\} \operatorname{NMR}\left(75 \mathrm{MHz}, \mathrm{CDCl}_{3}\right): \delta 157.1\left(C_{\text {carbene }}\right),[131.2,131.1,130.7$, $129.8,129.0,128.6,127.7,127.2,126.2,125.9,125.6,125.5,124.7,123.1,122.5$, 


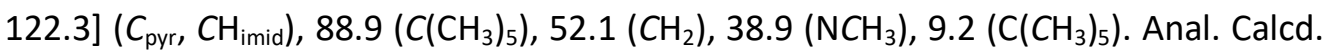
for $\mathrm{C}_{31} \mathrm{H}_{31} \mathrm{~N}_{2} \mathrm{IrCl}_{2}(694.71 \mathrm{~g} / \mathrm{mol}): \mathrm{C}, 53.59 ; \mathrm{H}, 4.49 ; \mathrm{N}, 4.03$. Found: $\mathrm{C}, 53.67 ; \mathrm{H}, 4.27$; $\mathrm{N}$, 4.37. Electrospray MS. (Cone $20 \mathrm{~V})\left(\mathrm{m} / \mathrm{z}\right.$, fragment): $659.3[\mathrm{M}-\mathrm{Cl}]^{+}$. HRMS ESI-TOF-MS (positive mode): $\left[\mathrm{M}-\mathrm{Cl}^{+}\right.$monoisotopic peak 659.1803; calc. $659.1798, \varepsilon_{\mathrm{r}}: 0.8 \mathrm{ppm}$.

\section{Synthesis of $8 C^{\prime}$}

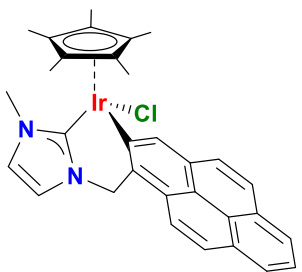

Under the exclusion of light, imidazolium salt C $(95 \mathrm{mg}$, $0.25 \mathrm{mmol}$ ) and $\mathrm{Ag}_{2} \mathrm{O}$ (58.5 $\mathrm{mg}, 0.25 \mathrm{mmol}$ ) were mixed in $10 \mathrm{~mL}$ of acetonitrile in a round-bottom flask, and the suspension was stirred for $5 \mathrm{~h}$ at room temperature. $\left[\mathrm{Cp}^{*} \mathrm{IrCl}_{2}\right]_{2}(100 \mathrm{mg}, 0.125 \mathrm{mmol})$ and $\mathrm{KCl}(243 \mathrm{mg}, 3.25 \mathrm{mmol})$ were added, and the reaction mixture was stirred at room temperature for $15 \mathrm{~h}$. Acetonitrile was removed under reduced pressure, and the mixture was suspended in dichloromethane. The insoluble salts were removed by filtration, and the crude product was purified by flash chromatography, producing 8C' as a crystalline orange powder. Yield: $80 \mathrm{mg}(49 \%) .{ }^{1} \mathrm{H} \mathrm{NMR}\left(400 \mathrm{MHz}^{\prime} \mathrm{CDCl}_{3}\right.$ ) $\delta 8.67\left(\mathrm{~s}, 1 \mathrm{H}, \mathrm{CH}_{\text {pyr }}\right), 8.20\left(\mathrm{~d},{ }^{3} \mathrm{~J}_{\mathrm{H}-\mathrm{H}}=9.4 \mathrm{~Hz}, 1 \mathrm{H}, \mathrm{CH}_{\text {pyr }}\right), 7.98\left(\mathrm{~m}, 6 \mathrm{H}, \mathrm{CH}_{\text {pyr }}\right), 7.07$ (d, $\left.{ }^{3} J_{\mathrm{H}-\mathrm{H}}=2.0 \mathrm{~Hz}, 1 \mathrm{H}, \mathrm{CH}_{\text {imid }}\right), 6.88\left(\mathrm{~d},{ }^{3} \mathrm{~J}_{\mathrm{H}-\mathrm{H}}=2.0 \mathrm{~Hz}, 1 \mathrm{H}, \mathrm{CH}_{\text {imid }}\right), 5.99,5.18(\mathrm{AB}$, $\left.{ }^{2} J_{A B}=15.1 \mathrm{~Hz}, 2 \mathrm{H}, \mathrm{CH}_{2}\right), 3,94\left(\mathrm{~s}, 3 \mathrm{H}, \mathrm{NCH}_{3}\right), 1.71\left(\mathrm{~s}, 15 \mathrm{H}, \mathrm{CH}_{3, \mathrm{Cp}}\right) .{ }^{13} \mathrm{C}\left\{{ }^{1} \mathrm{H}\right\} \mathrm{NMR}$ $\left(101 \mathrm{MHz}, \mathrm{CDCl}_{3}\right): \delta 156.3$ (C Carbene), [142.3, 141.2, 132.6, 130.9, 130.3, 129.8, 127.8, $126.1,125.5,125.2,125.1,124.3,124.0,123.5,121.9,121.6,121.2,120.6]\left(C_{\text {pyr }}\right.$,

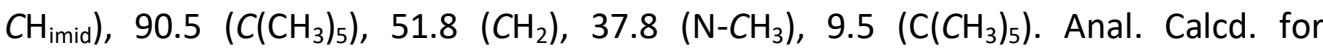
$\mathrm{C}_{31} \mathrm{H}_{30} \mathrm{~N}_{2} \mathrm{IrCl}$ (658.26 g/mol): C, 56.56; $\mathrm{H}, 5.80 ; \mathrm{N}, 4.25$. Found: $\mathrm{C}, 56.16 ; \mathrm{H}, 5.35$; $\mathrm{N}$, 4.19. HRMS ESI-TOF-MS (positive mode): [M - $\mathrm{Cl}]^{+}$monoisotopic peak 623.2044; calc. $623.2040, \varepsilon_{\mathrm{r}}: 0.6 \mathrm{ppm}$.

\section{Synthesis of $10 \mathrm{H}$}

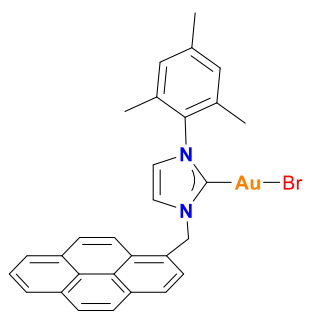

In a Pyrex tube ${ }^{\circledR}$, imidazolium salt $\mathbf{H}(100 \mathrm{mg}, 0.2 \mathrm{mmol})$, $\left[\mathrm{AuCl}\left(\mathrm{SMe}_{2}\right)\right](60.7 \mathrm{mg}, 0.206 \mathrm{mmol}), \mathrm{K}_{2} \mathrm{CO}_{3}(28.5 \mathrm{mg}$, $0.206 \mathrm{mmol}$ ) and $2.4 \mathrm{~mL}$ of acetone were heated at $60{ }^{\circ} \mathrm{C}$ for 3 h. Then, the solvent was removed under vacuum, dichloromethane was added $(10 \mathrm{~mL})$ and the mixture was filtered through silica. The pad of silica was washed with dichloromethane $(10 \mathrm{~mL})$. The solvent was reduced to approximately $2 \mathrm{~mL}$ and pentane was added, affording a white solid, which was 
filtered and dried in vacuum. Yield: $\left.90 \mathrm{mg}(66 \%) .{ }^{1} \mathrm{H} \mathrm{NMR} \mathrm{(300} \mathrm{MHz,} \mathrm{CDCl}\right) \delta 8.35$ $\left(\mathrm{d},{ }^{3} \mathrm{~J}=9.2 \mathrm{~Hz}, 1 \mathrm{H}, \mathrm{CH}_{\text {pyr }}\right), 8.30-7.98\left(\mathrm{~m}, 8 \mathrm{H}, \mathrm{CH}_{\text {pyr }}\right), 6.97\left(\mathrm{~s}, 2 \mathrm{H}, \mathrm{CH}_{\text {mes }}\right), 6.80$ (d, $\left.{ }^{3} J_{\mathrm{H}-\mathrm{H}}=2.0 \mathrm{~Hz}, 1 \mathrm{H}, \mathrm{CH}_{\text {imid }}\right), 6.73\left(\mathrm{~d},{ }^{3} \mathrm{~J}_{\mathrm{H}-\mathrm{H}}=2.0 \mathrm{~Hz}, 1 \mathrm{H}, \mathrm{CH}_{\text {imid }}\right), 6.21,\left(\mathrm{~s}, 2 \mathrm{H}, \mathrm{CH}_{2}\right) 2.34(\mathrm{~s}$, $\left.3 \mathrm{H}, \mathrm{CH}_{3, \text { mes }}\right), 2.06\left(\mathrm{~s}, 6 \mathrm{H}, \mathrm{CH}_{3, \text { mes }}\right) .{ }^{13} \mathrm{C}\left\{{ }^{1} \mathrm{H}\right\} \mathrm{NMR}\left(75 \mathrm{MHz}, \mathrm{CDCl}_{3}\right) \delta 176.1\left(C_{\text {carbene }}\right)$, $[139.9,134.9,134.8,132.4,131.3,130.7,129.6,129.5,129.2,128.5,128.0,127.4$, $127.3,126.6,126.2,126.1,125.4,125.1,124.6,122.5,122.3,120.2]\left(C_{\mathrm{Pyr}}, C_{\mathrm{imid}}\right.$, $\left.C_{\text {mes }}\right), 53.7\left(\mathrm{CH}_{2}\right), 21.3\left(\mathrm{CH}_{3}\right.$,mes $), 18.0\left(\mathrm{CH}_{3, \text { mes }}\right)$. Anal. Calcd for $\mathrm{C}_{29} \mathrm{H}_{24} \mathrm{~N}_{2} \mathrm{AuBr} \cdot 0.5 \mathrm{C}_{5} \mathrm{H}_{12}$ : C, 52.98; H, 4.20; N, 3.92. Found: C, 53.27; H, 3.76; N, 4.15. The calculated elemental analysis fits with the experimental with the addition of half a molecule of $n$-pentane that is also observed in the ${ }^{1} \mathrm{H}$ NMR of complex $10 \mathrm{H}$. For the MS analyses, complex $\mathbf{1 0 H}$ was dissolved in $\mathrm{MeOH}$ together with potassium iodide, which allows the ionization of the molecule. Electrospray MS (Cone $20 \mathrm{~V}$ ) (m/z, fragment): $763.03[\mathrm{M}-\mathrm{Br}+\mathrm{I}+\mathrm{K}]^{+}$. HRMS ESI-TOF-MS (positive mode): $[\mathrm{M}-\mathrm{Br}+\mathrm{I}+\mathrm{K}]^{+}$monoisotopic peak 763.0305; calc. 763.0287, عr: 2.4 ppm.

\section{Synthesis of 111}

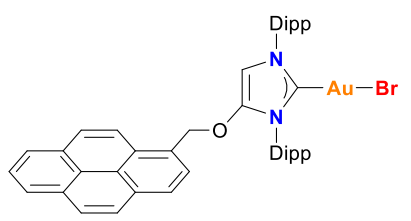

In a Pyrex ${ }^{\circledR}$ tube, imidazolium salt I (218 mg, $0.31 \mathrm{mmol}$ ), $\left[\mathrm{AuCl}\left(\mathrm{SMe}_{2}\right)\right](91.8 \mathrm{mg}, 0.31 \mathrm{mmol}), \mathrm{K}_{2} \mathrm{CO}_{3}(64.3 \mathrm{mg}$, $0.465 \mathrm{mmol}$ ) and $2 \mathrm{~mL}$ of acetone were heated at $60{ }^{\circ} \mathrm{C}$ for $18 \mathrm{~h}$. Then, the solvent was removed under vacuum, dichloromethane was added $(10 \mathrm{~mL})$ and the mixture was filtered through silica. The pad of silica was washed with dichloromethane $(10 \mathrm{~mL})$. The solvent was reduced to approximately $2 \mathrm{~mL}$ and pentane was added, affording an orange solid, which was filtered and dried under vacuum. Yield: $185 \mathrm{mg}$ (66 \%). ${ }^{1} \mathrm{H}$ NMR (300 MHz, $\left.\mathrm{CDCl}_{3}\right) \delta 8.23\left(\mathrm{~d},{ }^{3} \mathrm{~J}_{\mathrm{H}-\mathrm{H}}=7.9 \mathrm{~Hz}, 2 \mathrm{H}, \mathrm{CH}_{\text {pyr }}\right), 8.15-7.99(\mathrm{~m}$, $\left.6 \mathrm{H}, \mathrm{CH}_{\text {pyr }}\right), 7.90\left(\mathrm{~d},{ }^{3} \mathrm{~J}_{\mathrm{H}-\mathrm{H}}=7.9 \mathrm{~Hz}, 1 \mathrm{H}, \mathrm{CH}_{\text {pyr }}\right), 7.54-7.38\left(\mathrm{~m}, 2 \mathrm{H}, \mathrm{CH}_{\text {arom }}\right), 7.27-7.13$ $\left(\mathrm{m}, 4 \mathrm{H}, \mathrm{CH}_{\text {arom }}\right), 6.42\left(\mathrm{~s}, 1 \mathrm{H}, \mathrm{CH}_{\text {imid }}\right), 5.74\left(\mathrm{~s}, 2 \mathrm{H}, \mathrm{CH}_{2}\right), 2.62\left(\mathrm{~m}, 4 \mathrm{H}, \mathrm{CH}_{\text {iPr }}\right) 1.33$ (d+d, $12 \mathrm{H}, \mathrm{CH}_{3, \mathrm{Pr}}$ ), 1.19 (d, ${ }^{3} \mathrm{~J}_{\mathrm{H}-\mathrm{H}}=6.9 \mathrm{~Hz}, 6 \mathrm{H}, \mathrm{CH}_{3, \mathrm{Pr}}$ ), $1.12\left(\mathrm{~d},{ }^{3} \mathrm{~J}_{\mathrm{H}-\mathrm{H}}=6.9 \mathrm{~Hz}, 6 \mathrm{H}, \mathrm{CH}_{3, \mathrm{Pr}}\right.$ ). ${ }^{13} \mathrm{C}\left\{{ }^{1} \mathrm{H}\right\} \operatorname{NMR}\left(75 \mathrm{MHz}, \mathrm{CDCl}_{3}\right) \delta 173.7$ ( $\left.C_{\text {carbene }}\right), 147.9\left(C_{\text {imid }}\right),[146.2,145.7]\left(C_{\text {arom }}\right)$, $[134.6,132.3,131.2,130.8,130.6,130.3,129.4,128.7,128.4,127.2,126.8,126.6$, $126.4,126.0,125.8,124.9,124.5,124.2,124.1,122.1]\left(C_{\mathrm{pyr}}, C_{\text {arom }}\right), 100.9\left(C_{\mathrm{imid}}\right)$, $73.1\left(\mathrm{CH}_{2}\right),[29.1,28.7]\left(\mathrm{CH}_{i \mathrm{Pr}}\right),[24.6,24.4,24.1,23.6]\left(\mathrm{CH}_{3, \mathrm{Pr}}\right)$. Anal. Calcd. for for $\mathrm{C}_{44} \mathrm{H}_{46} \mathrm{~N}_{2} \mathrm{OAuBr}(895.72 \mathrm{~g} / \mathrm{mol}): \mathrm{C}, 59.00 ; \mathrm{H}, 5.18 ; \mathrm{N}, 3.13$ Found: C, 59.3; H, 5.42; $\mathrm{N}, 3.09$. For the $\mathrm{MS}$ analyses, complex $\mathbf{1 1 l}$ was dissolved in $\mathrm{MeOH}$ together with potassium bromide, which allows the ionization of the molecule. Electrospray MS (Cone $20 \mathrm{~V}$ ) (m/z, fragment): $935.0[\mathrm{M}+\mathrm{K}]^{+}$. 


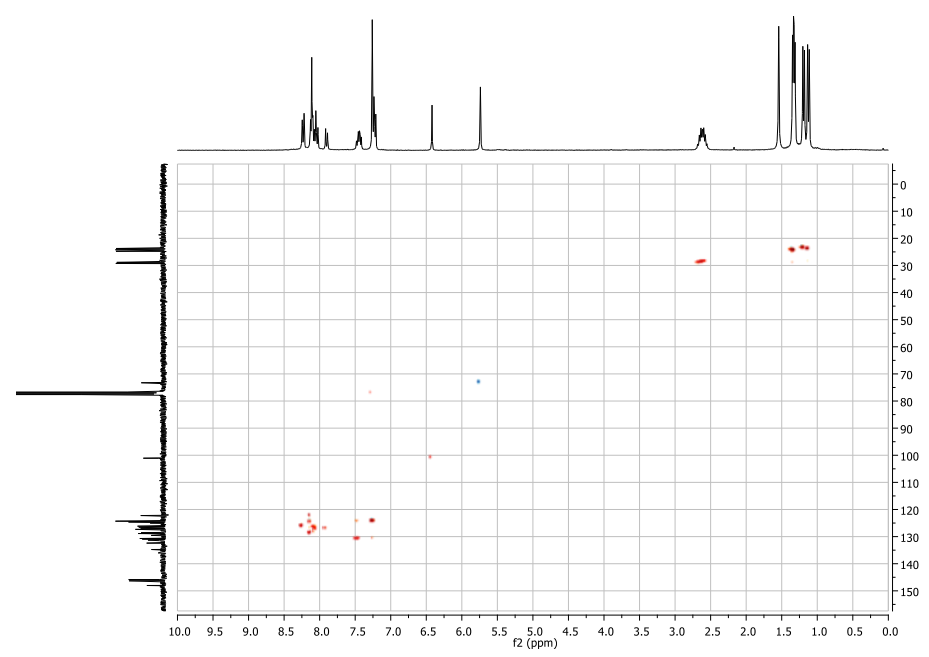

${ }^{1} \mathrm{H}-{ }^{13} \mathrm{C} \mathrm{HSQC} \mathrm{NMR} \mathrm{spectrum}\left({ }^{1} \mathrm{H}, 300 \mathrm{MHz}\right.$ and $\left.{ }^{13} \mathrm{C}, 75 \mathrm{MHz}\right)$ of $11 \mathrm{l}$ in $\mathrm{CDCl}_{3}$

\subsubsection{Synthesis and characterisation of hybrid M-rGO materials}

General procedure: In a round bottom flask were introduced the appropriate amounts of $\mathrm{rGO}$ and dichloromethane. The mixture was sonicated for $30 \mathrm{~min}$ and then the suitable amount of the metal complex was added and the suspension was further sonicated for $15 \mathrm{~min}$ and then stirred at room temperature for 24-72 hours. After this time the black solid was filtered and further washed with dichloromethane. The exact amount of metal loaded on the surface of rGO was determined by ICP-MS analysis and the material was characterised by TEM. XPS and UV/Vis analysis were also performed for selected examples.

\section{Synthesis of 1A-rGO}

Following the general procedure, 1A-rGO was obtained from $180 \mathrm{mg}$ rGO, $20 \mathrm{mg}$ of $1 \mathrm{~A}$ and stirring in $200 \mathrm{~mL}$ of dichloromethane for 24 hours. The ICP-MS analysis accounted for a $1.1 \mathrm{wt} \%$ of $\mathbf{1 A}$ on the surface of the material $\mathbf{1 A - r G O}$. 


\section{$\underline{\text { TEM Images }}$}

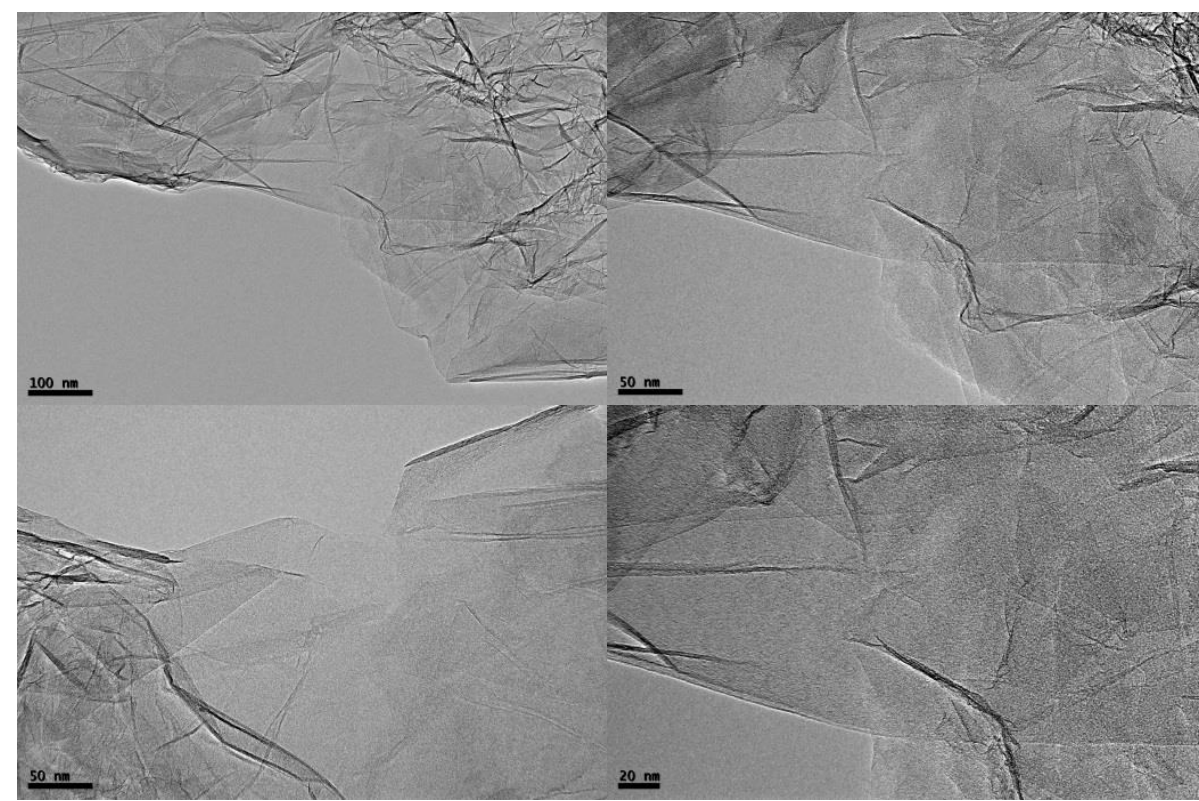

Figure 6.1 TEM images of $1 \mathrm{~A}-\mathrm{rGO}$ at different magnifications

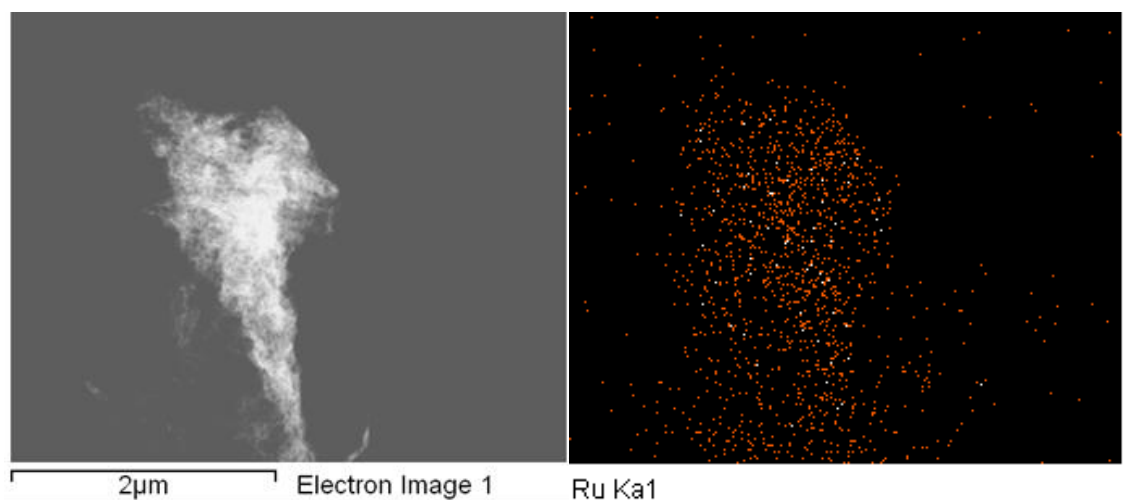

Figure 6.2 STEM (left) and EDS elemental mapping (right) images showing the homogeneous distribution of ruthenium on the hybrid material 1 A-rGO 


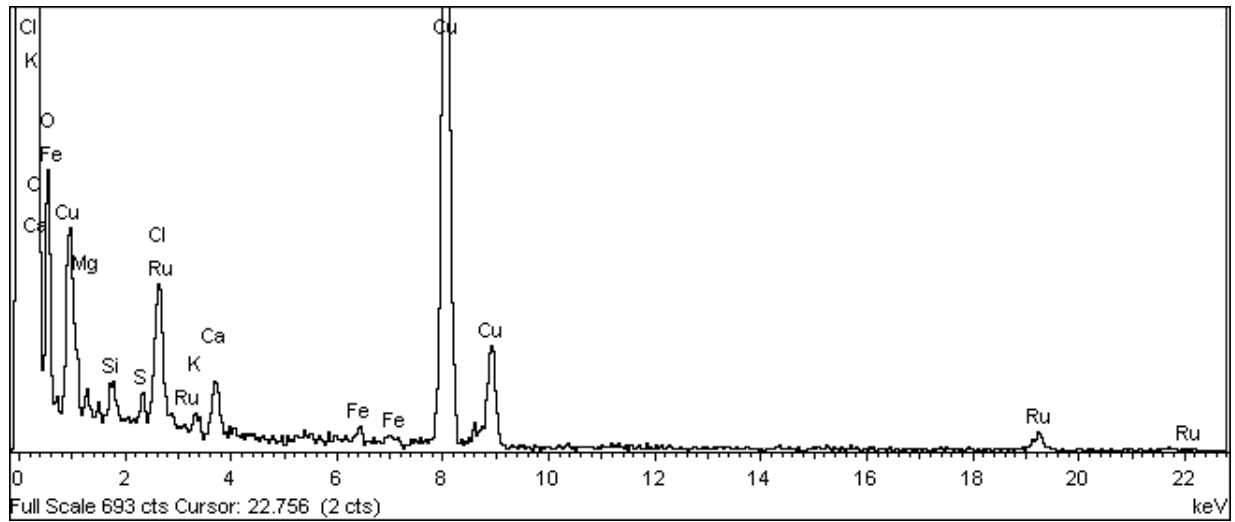

Figure 6.3 EDS spectrum of 1A-rGO

\section{Synthesis of 2B-rGO}

Following the general procedure, 2B-rGO was obtained from $180 \mathrm{mg}$ rGO, $20 \mathrm{mg}$ of 2B and stirring in $200 \mathrm{~mL}$ of dichloromethane for 24 hours. The ICP-MS analysis accounted for a $3.2 \mathrm{wt} \%$ of $\mathbf{2 B}$ on the surface of the material $\mathbf{2 B}-\mathbf{B G O}$.

\section{$\underline{\text { TEM Images }}$}

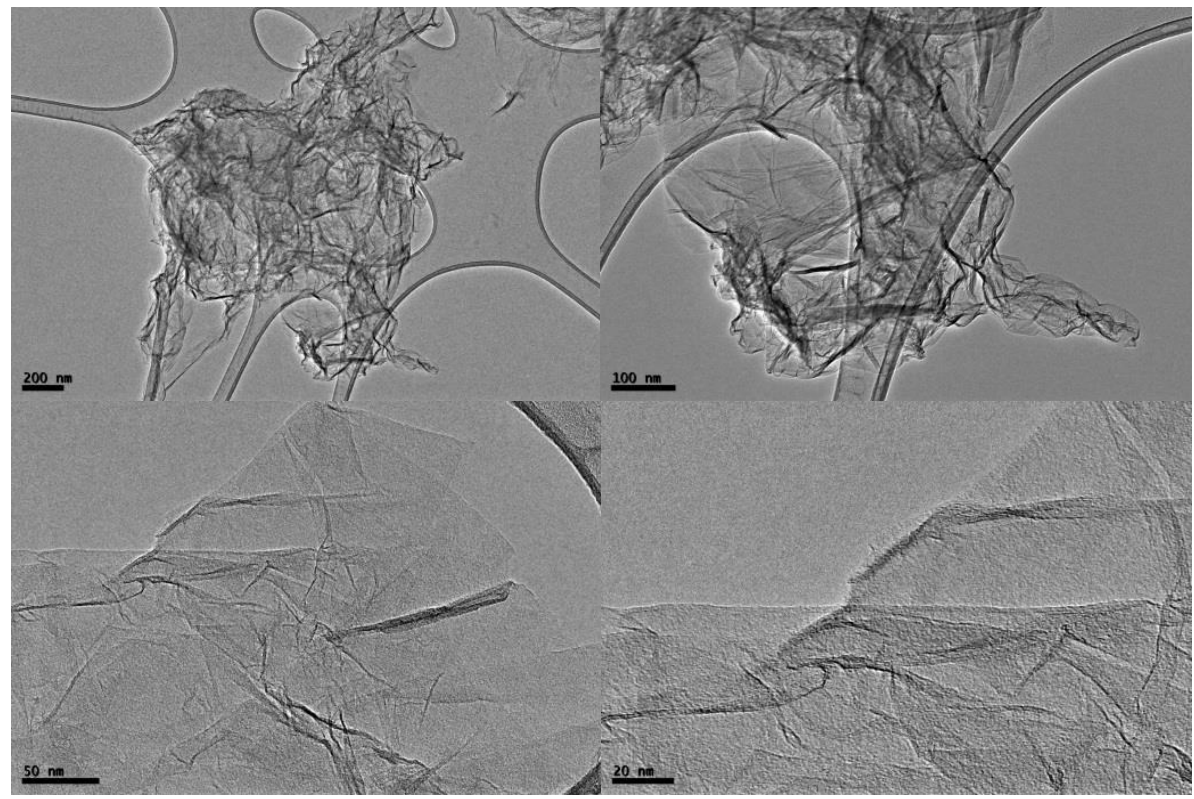

Figure 6.4 TEM images of 2B-rGO at different magnifications 


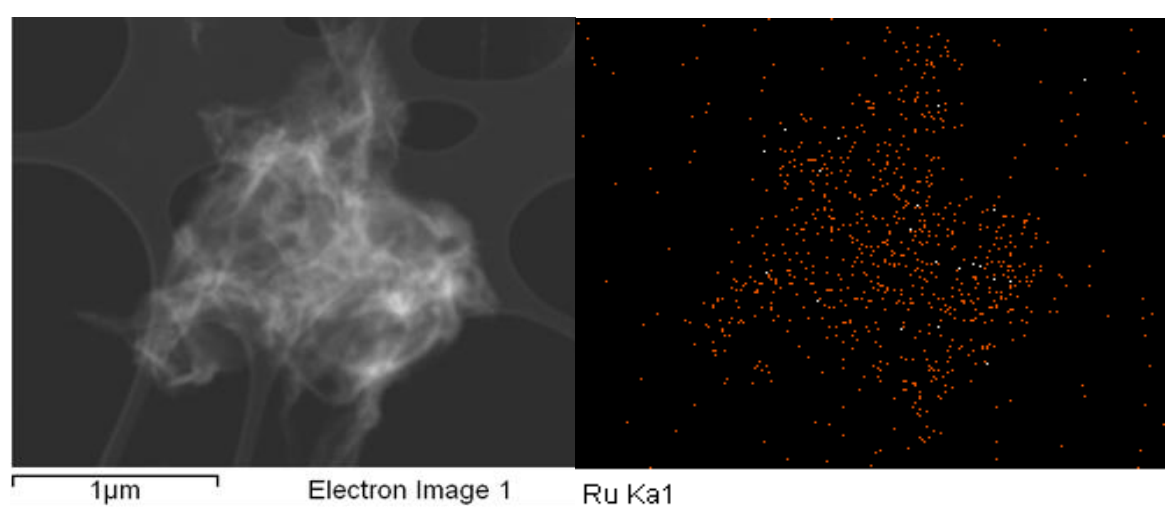

Figure 6.5 STEM (left) and EDS elemental mapping (right) images showing the homogeneous distribution of ruthenium on the hybrid material 2B-rGO

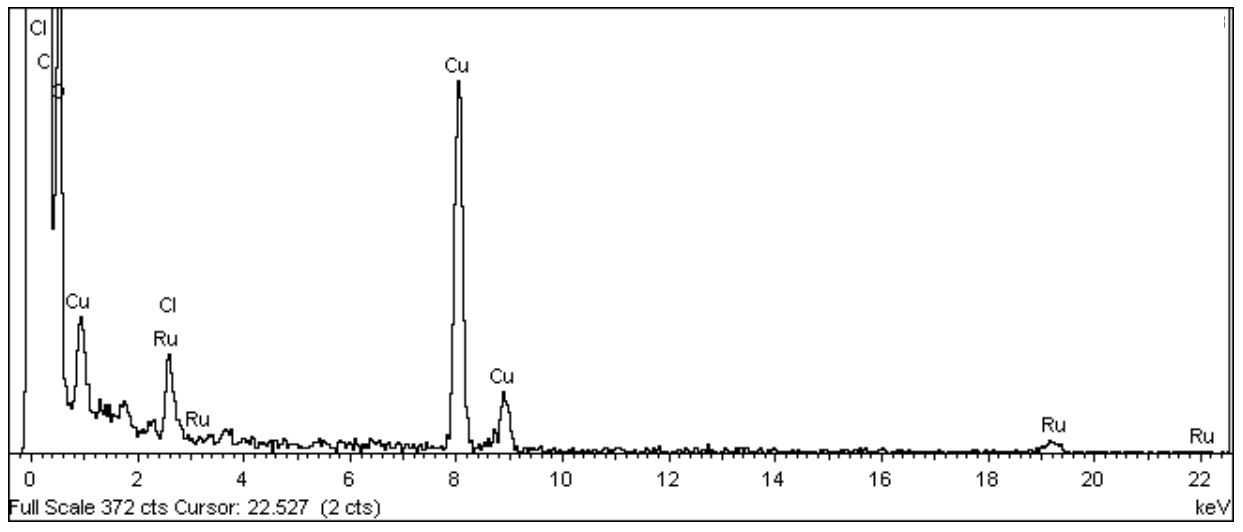

Figure 6.6 EDS spectrum of 2B-rGO

\section{$\underline{\text { UV/Vis spectroscopy }}$}

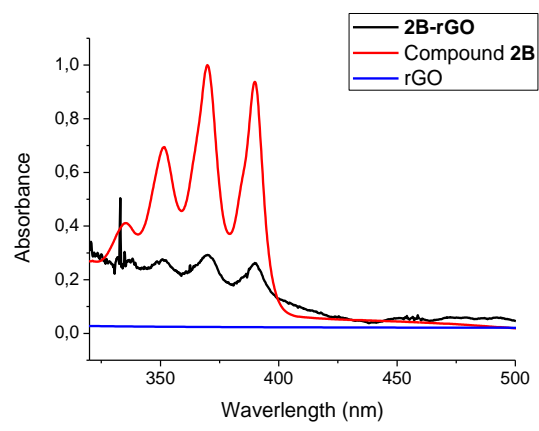

Figure 6.7 UV/Vis spectra for 2B, 2B-rGO and rGO. The samples were suspended in DMF and sonicated before recording the spectrum. The molecular complex was measured in a solution in $\mathrm{DMF}\left(10^{-6} \mathrm{M}\right)$ 


\section{Synthesis of 3C-rGO}

Following the general procedure, 3C-rGO was obtained from $540 \mathrm{mg} \mathrm{rGO}, 120 \mathrm{mg}$ of 3C and stirring in $360 \mathrm{~mL}$ of dichloromethane for 24 hours. The ICP-MS analysis accounted for a $9.1 \mathrm{wt} \%$ of $\mathbf{3 C}$ on the surface of the material $\mathbf{3 C}$-rGo.

\section{$\underline{\text { TEM images }}$}

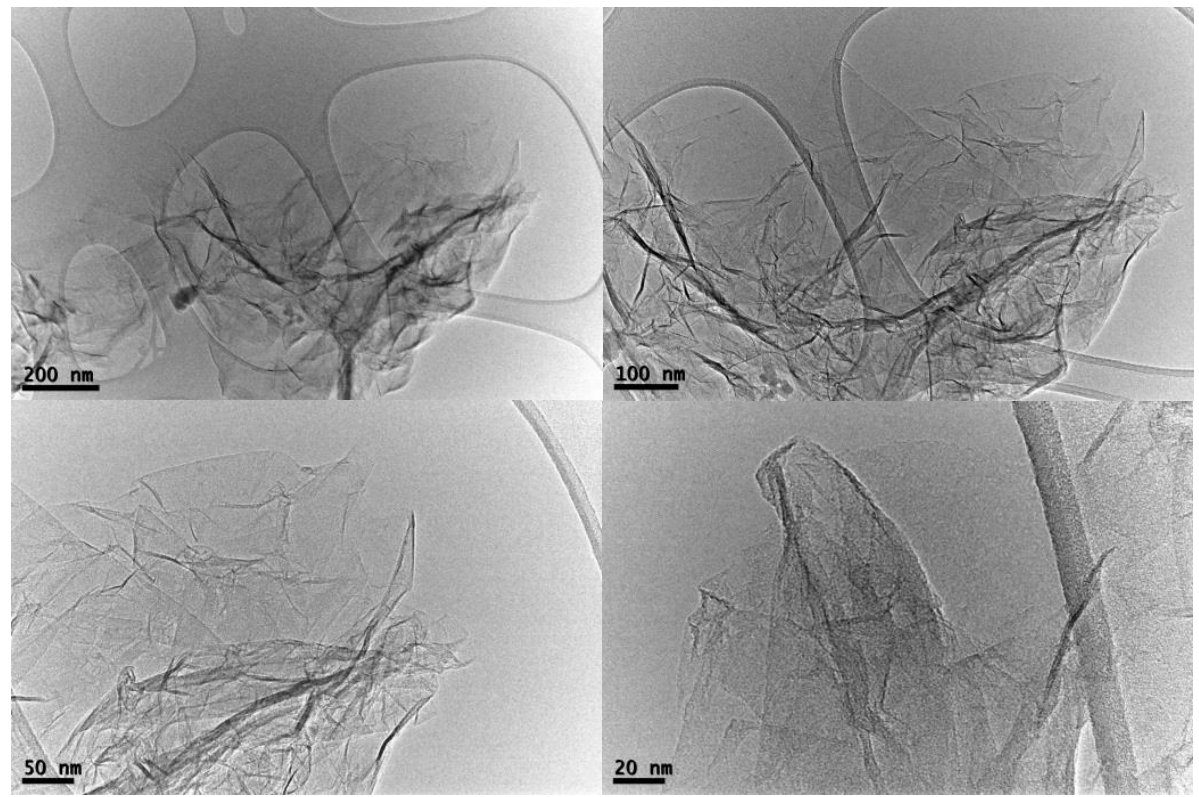

Figure 6.8 TEM images of 3C-rGO at different magnifications

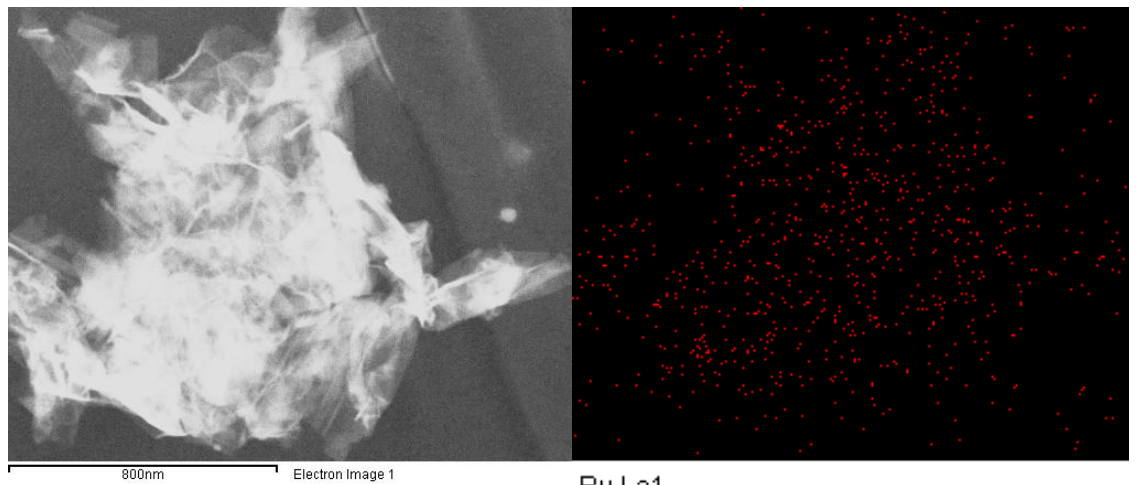

Ru La1

Figure 6.9 STEM (left) and EDS elemental mapping (right) images showing the homogeneous distribution of ruthenium on the hybrid material $3 \mathrm{C}-\mathrm{rGO}$ 


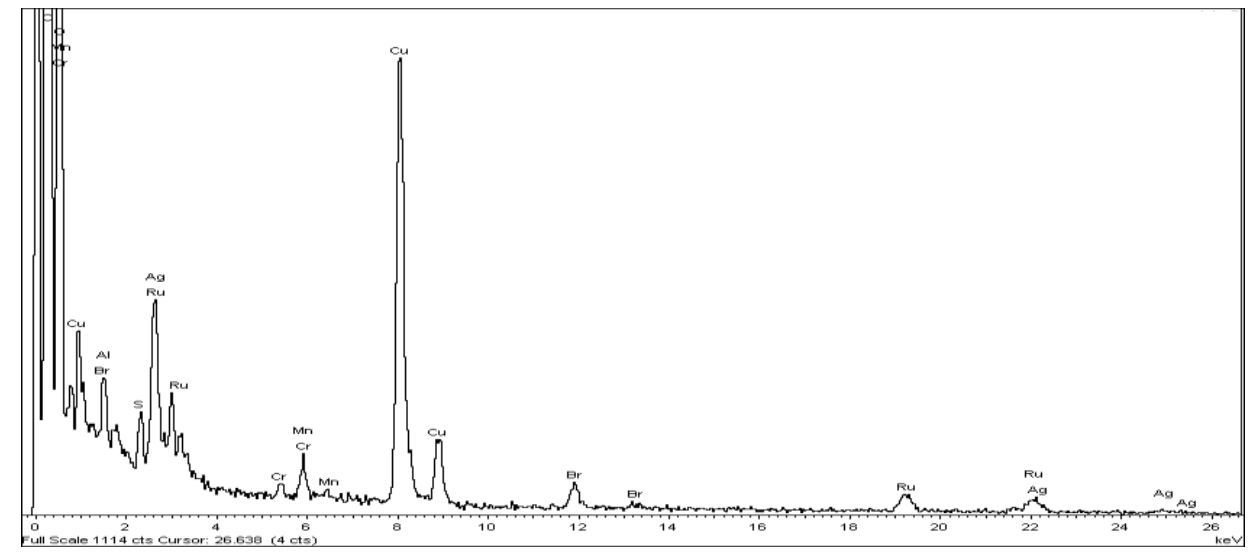

Figure 6.10 EDS spectrum of 3C-rGO

\section{UV/Vis spectroscopy}

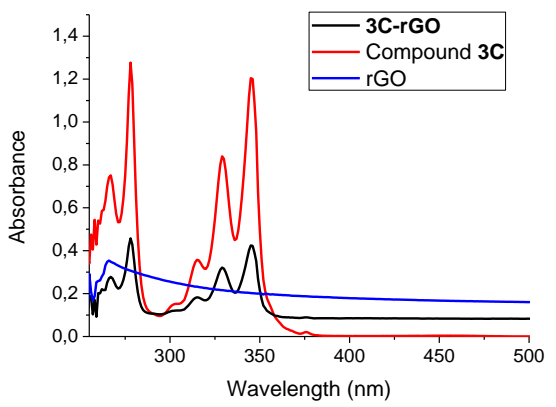

Figure 6.11 UV/Vis spectra for 3C, 3C-rGO and rGO. The samples were suspended in DMF and sonicated before recording the spectrum. The molecular complex was measured in a solution in $\mathrm{DMF}\left(10^{-6} \mathrm{M}\right)$ 


\section{$\underline{\text { XPS spectroscopy }}$}
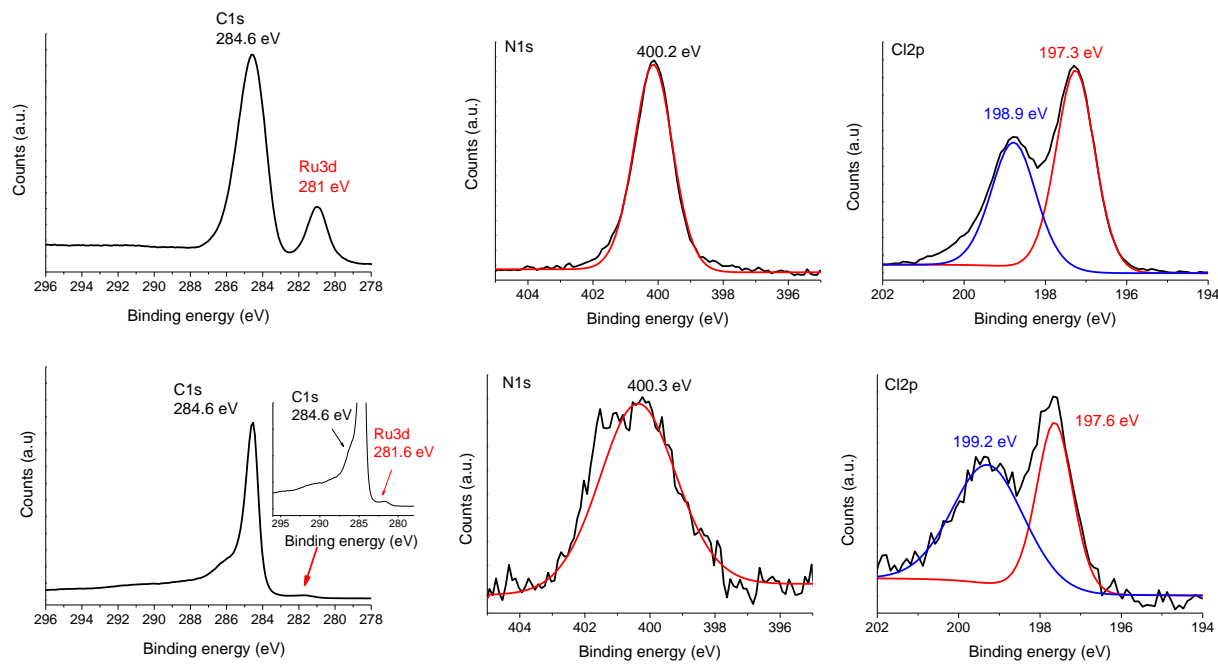

Figure 6.12 Comparative XPS analysis of molecular complex $3 \mathrm{C}$ (top) and hybrid material 3C-rGO (bottom) for the core-level peaks of Ru3d, Cl2p and N1s

\section{Synthesis of $8 \mathrm{C}-\mathrm{rGO}$}

Following the general procedure, 8C-rGO was obtained from $180 \mathrm{mg}$ rGO, $40 \mathrm{mg}$ of 3C and stirring in $120 \mathrm{~mL}$ of dichloromethane for 24 hours. The ICP-MS analysis accounted for a $6.5 \mathrm{wt} \%$ of $\mathbf{8 C}$ on the surface of the material $8 \mathrm{C}-\mathrm{rGO}$. 


\section{TEM images}

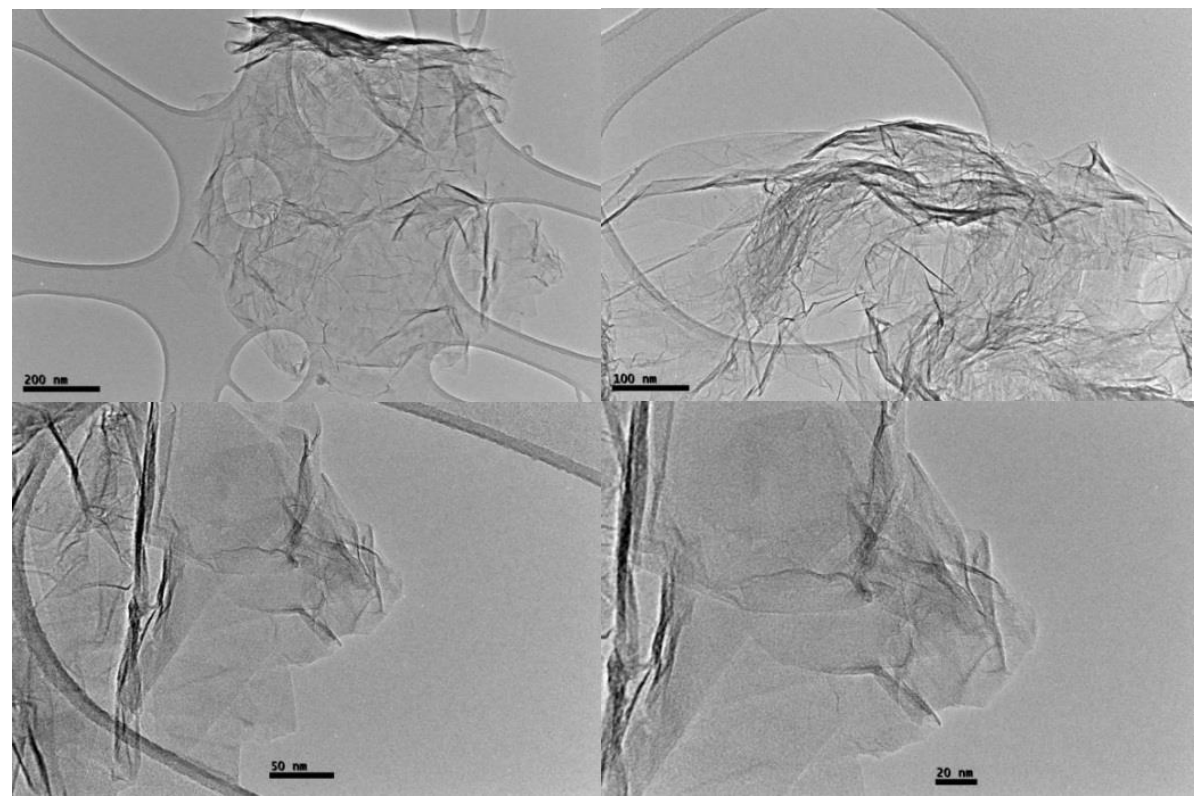

Figure 6.13 TEM images of 8C-rGO at different magnifications

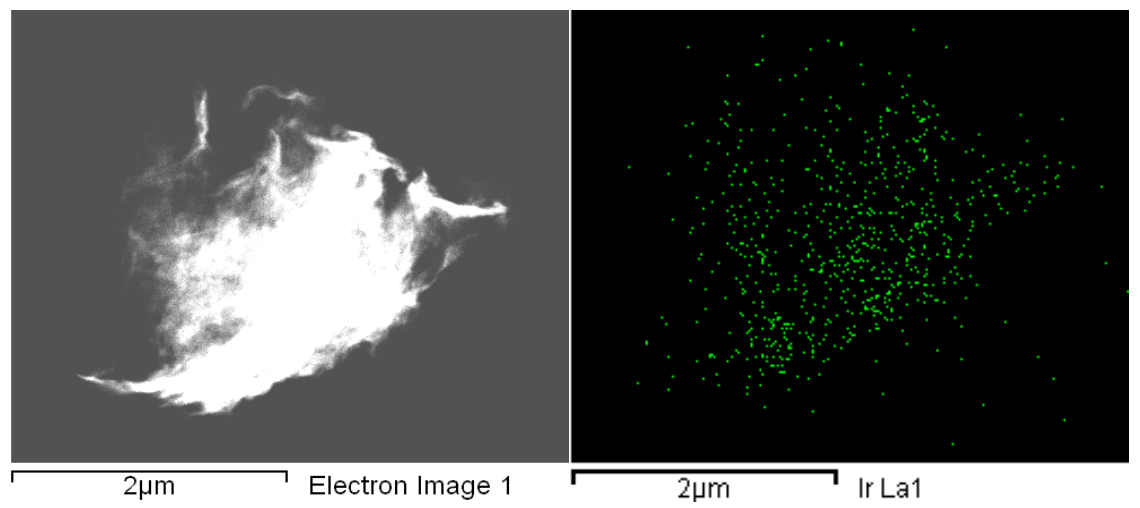

Figure 6.14 STEM (left) and EDS elemental mapping (right) images showing the homogeneous distribution of iridium on the hybrid material $8 \mathrm{C}$-rGO 


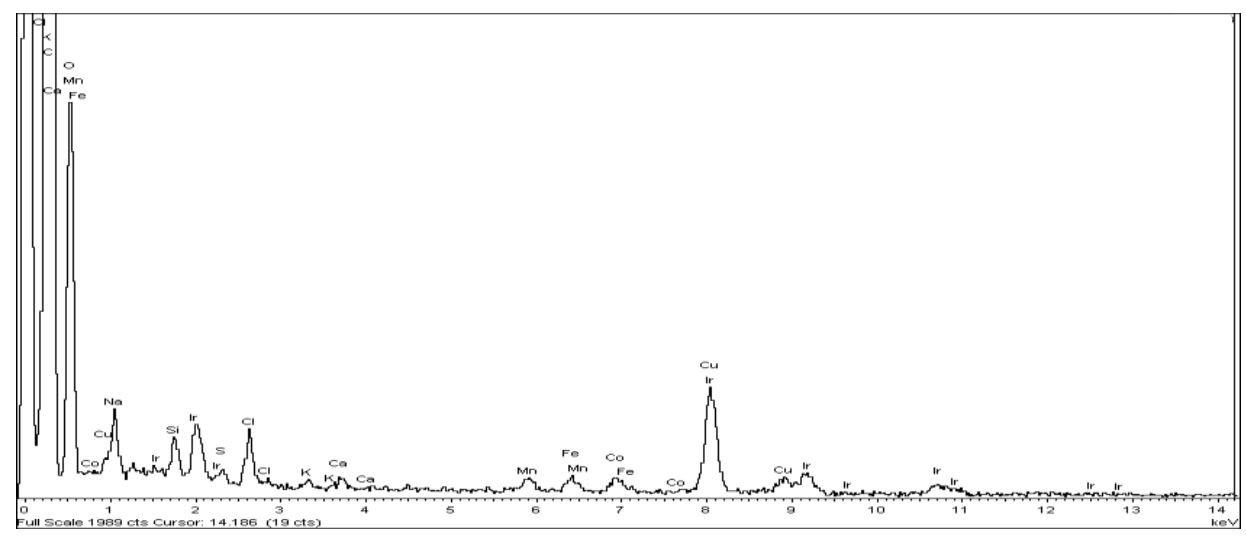

Figure 6.15 EDS spectrum of 8C-rGO

\section{$\underline{\text { UV/Vis spectroscopy }}$}

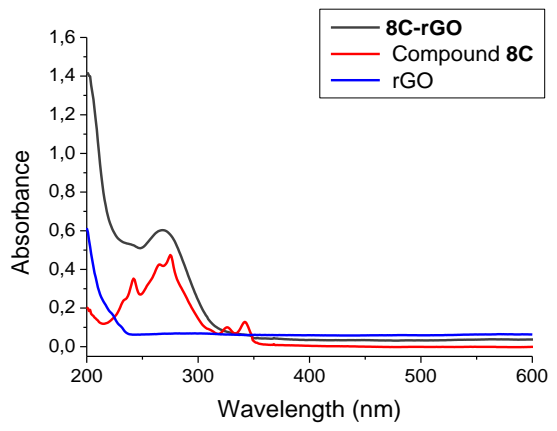

Figure 6.16 UV Vis spectra for 8C, 8C-rGO and rGO. The samples were suspended in DMF and sonicated before recording the spectrum. The molecular complex was measured in a solution in $\operatorname{DMF}\left(10^{-6} \mathrm{M}\right)$ 


\section{XPS spectroscopy}
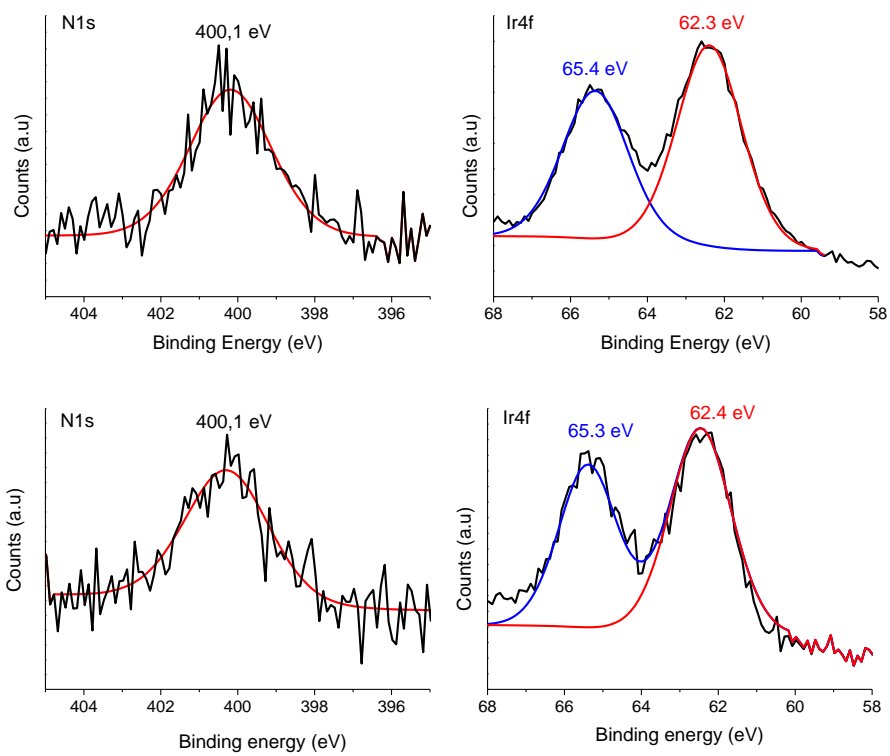

Figure 6.17 Comparative XPS analysis of molecular complex 8C (top) and hybrid material 8C-rGO (bottom) for the core-level peaks of Ir4f and N1s

\section{Synthesis of $10 \mathrm{H}-\mathrm{rGO}$}

Following the general procedure, $10 \mathrm{H}-\mathrm{rGO}$ was obtained from $180 \mathrm{mg} \mathrm{rGO}, 40 \mathrm{mg}$ of 3C and stirring in $120 \mathrm{~mL}$ of dichloromethane for 24 hours. The ICP-MS analysis accounted for a $0.9 \mathrm{wt} \%$ of $\mathbf{1 0 H}$ in the surface of the material $\mathbf{1 0 H}-\mathrm{rGO}$. 


\section{$\underline{\text { TEM Images }}$}

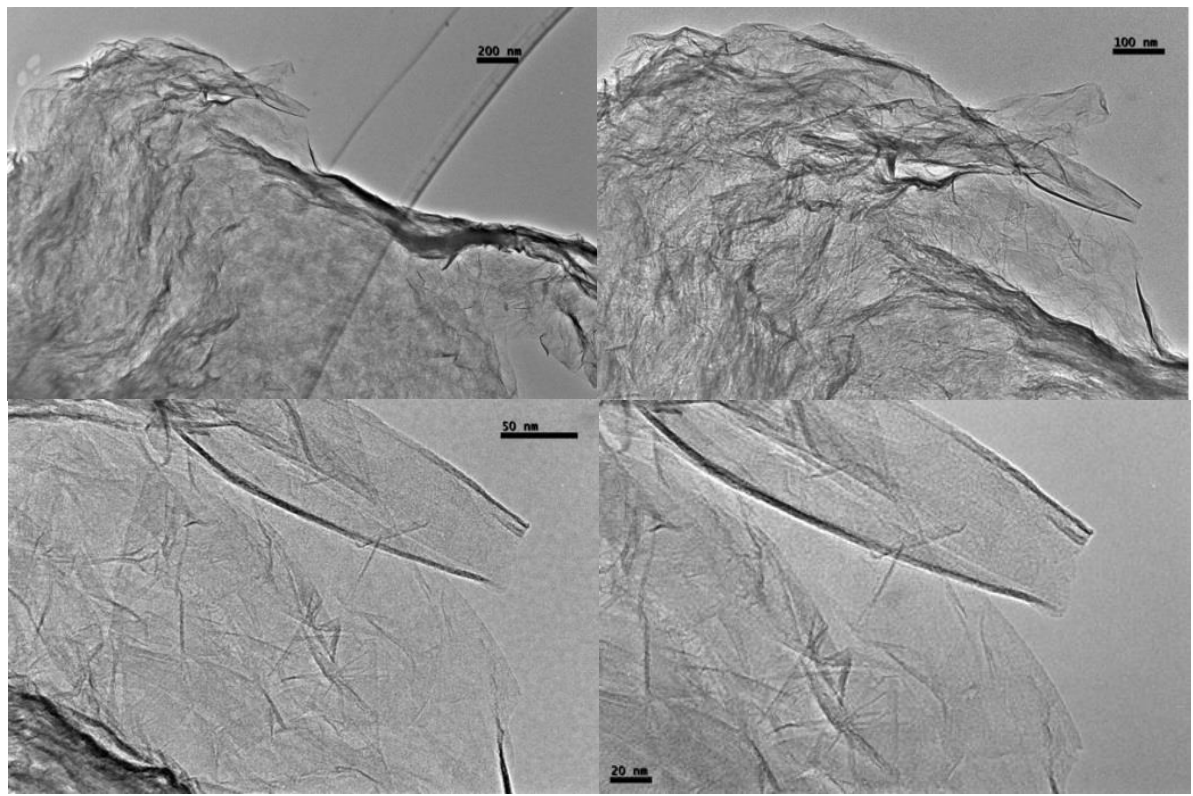

Figure 6.18 TEM images of $\mathbf{1 0 H}-\mathrm{rGO}$ at different magnifications

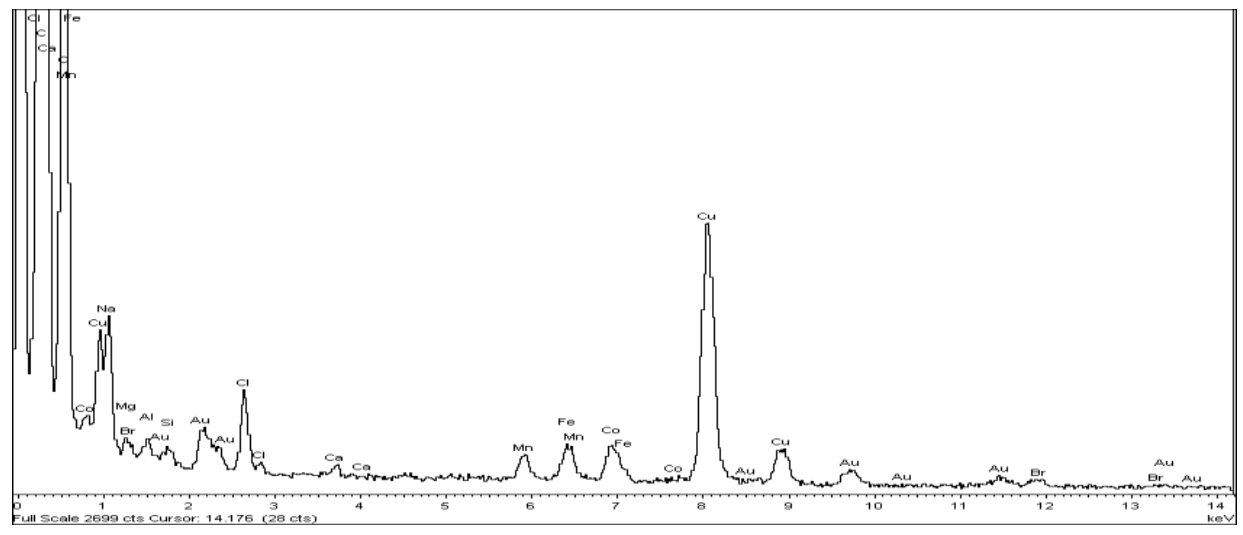

Figure 6.19 EDS spectrum of $10 \mathrm{H}-\mathrm{rGO}$ 


\section{XPS spectroscopy}
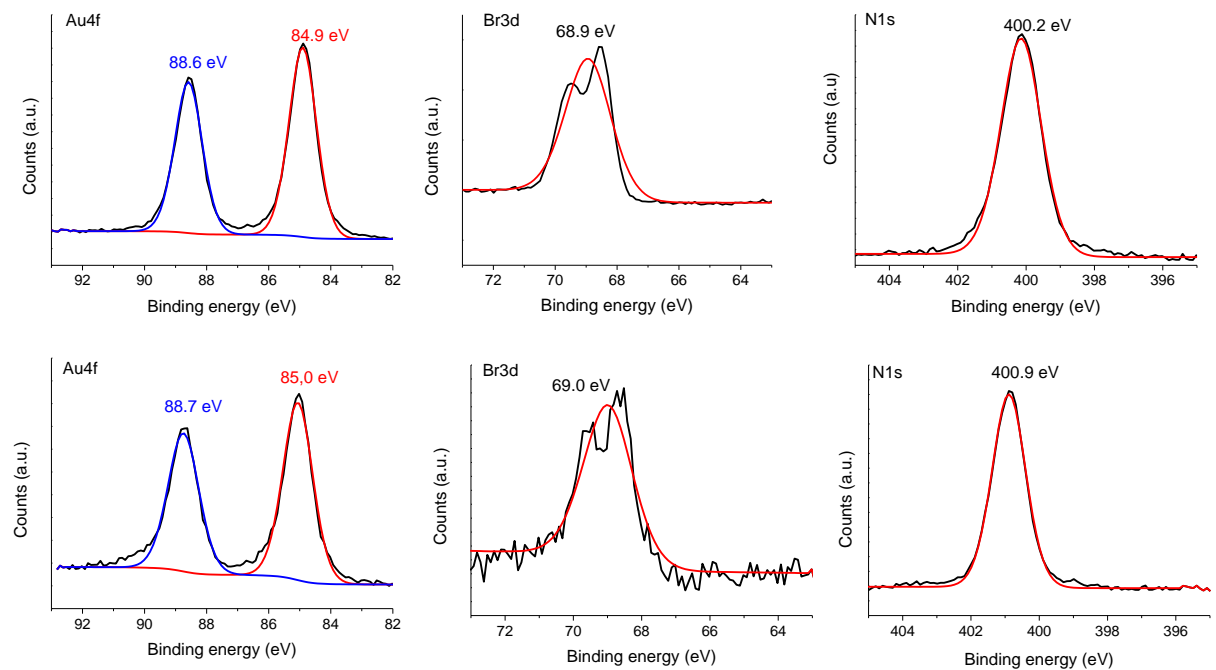

Figure 6.20 Comparative XPS analysis of molecular complex 10H (top) and hybrid material 10H-rGO (bottom) for the core-level peaks of Au4f, Br3d and N1s

\section{Synthesis of 11I-rGO}

Following the general procedure, 11l-rGO was obtained from $50 \mathrm{mg} \mathrm{rGO}, 11 \mathrm{mg}$ of 11 and stirring in $35 \mathrm{~mL}$ of dichloromethane for 24 hours. 


\section{$\underline{\text { TEM Images }}$}

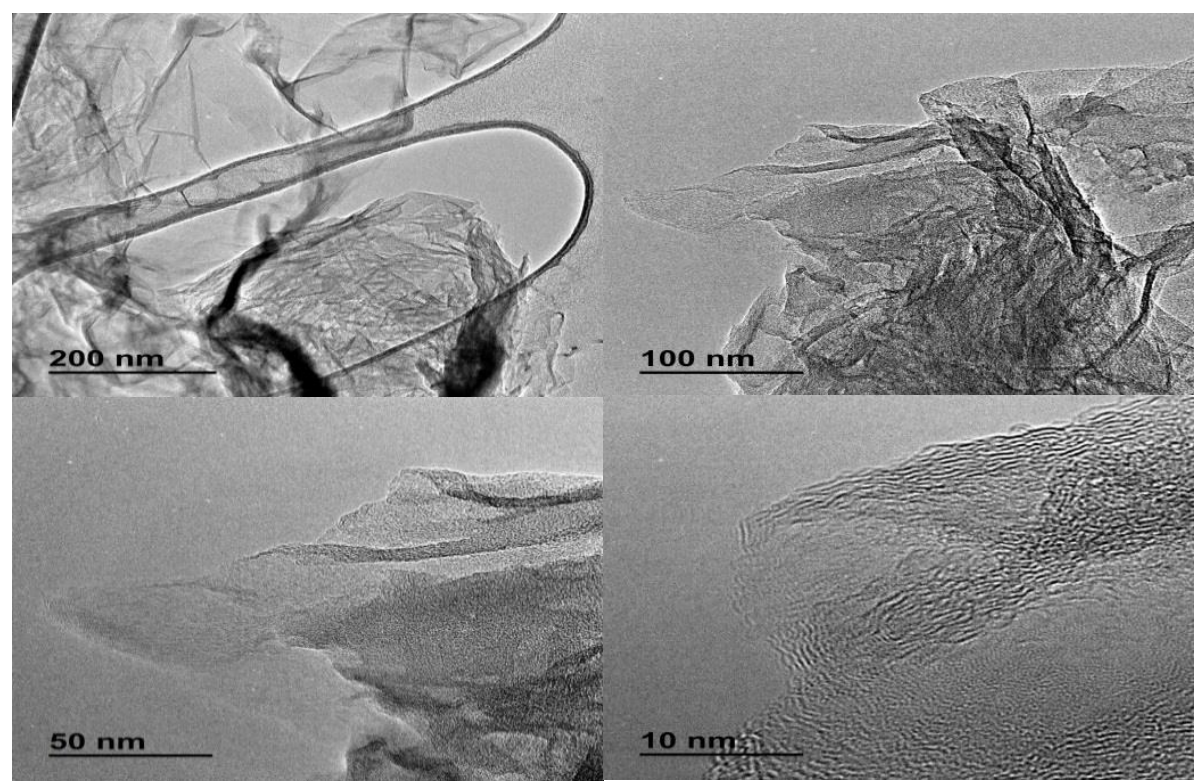

Figure 6.21 TEM images of 11I-rGO at different magnifications showing the absence of gold nanoparticles

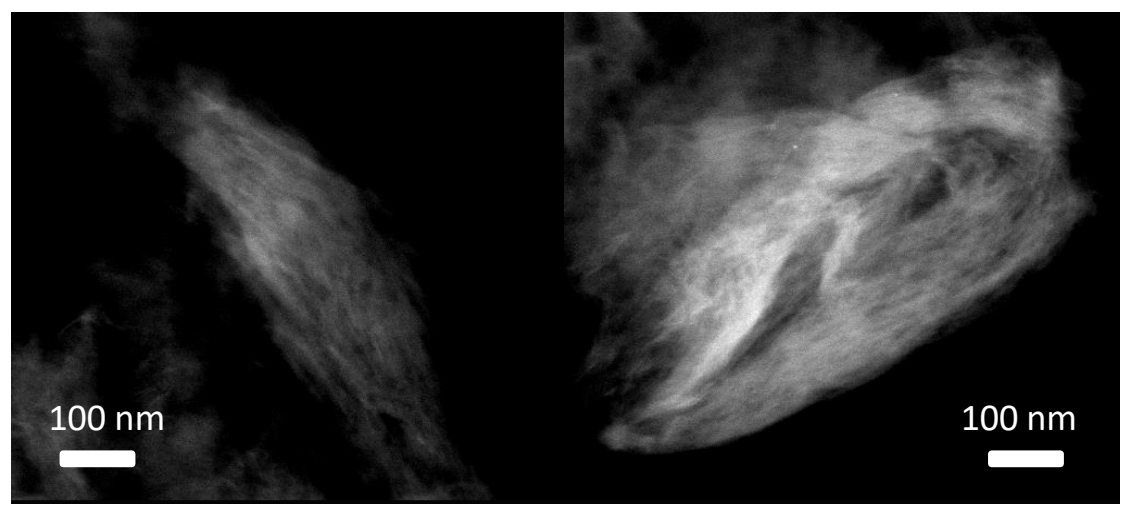

Figure 6.22 HAADF-STEM images of 11l-rGO showing the absence of gold nanoparticles 


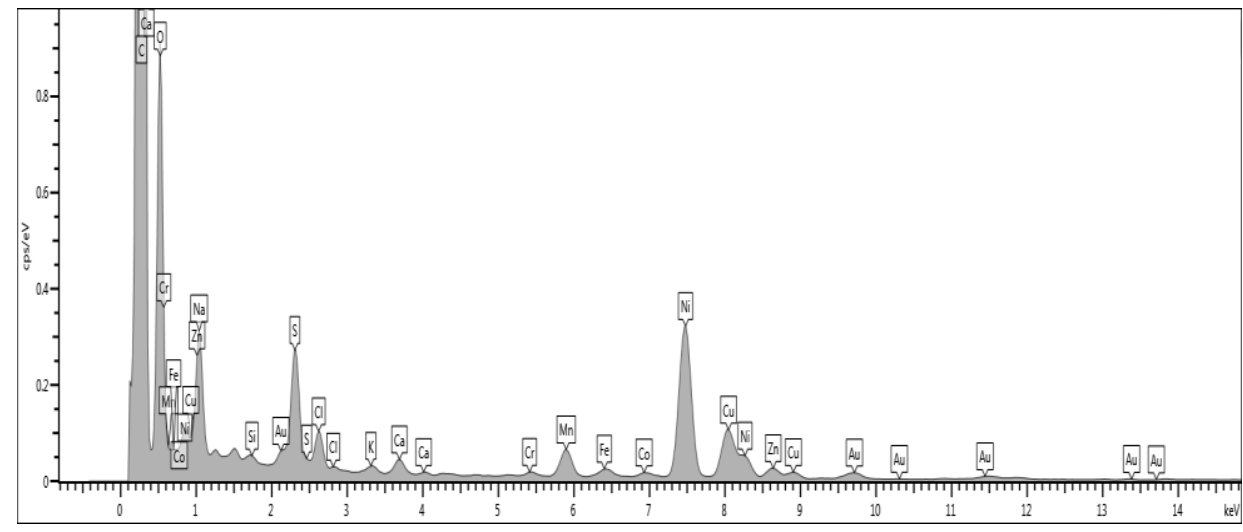

Figure 6.23 EDS spectrum of 11I-rGO showing the presence of gold on the surface of graphene

\section{Synthesis of 12I-rGO-NPs}

A suspension of $490 \mathrm{mg}$ of $\mathrm{rGO}$ in $350 \mathrm{~mL}$ of $\mathrm{CH}_{2} \mathrm{Cl}_{2}$ in a round bottom flask was sonicated for $30 \mathrm{~min}$. In a Schlenk flask and under the protection of light, complex $11 \mathrm{l}(150 \mathrm{mg}, 0.166 \mathrm{mmol})$ and Silver triflate $(56.4 \mathrm{mg}, 0.184 \mathrm{mmol})$ were dissolved in $8 \mathrm{~mL}$ of dry $\mathrm{CH}_{2} \mathrm{Cl}_{2}$ and the mixture was stirred at room temperature for $15 \mathrm{~min}$. Then the reaction was filtered through a long pad of celite. The solvent was reduced in the rotatory evaporator until ca. $5 \mathrm{~mL}$. Then, this mixture was added to the suspension of $\mathrm{rGO}$ in $\mathrm{CH}_{2} \mathrm{Cl}_{2}$ and was stirred at room temperature for 48 hours. The black solid was isolated by filtration and washed with $200 \mathrm{~mL}$ of $\mathrm{CH}_{2} \mathrm{Cl}_{2}$ affording the hybrid material 12I-rGO-NPs. The exact amount of gold content was determined by ICP-MS analysis. The results accounted for a $0.033 \mathrm{mg} \mathrm{Au} / 100 \mathrm{mg}$ rGO in the hybrid material 12I-rGO-NPs. The hybrid material was characterised by SEM, HRTEM, UV/Vis and XPS An aliquot of the in situ formed complex $\mathbf{1 2 I}$ was dried and analysed by ${ }^{1} \mathrm{H}$ NMR and ESI/MS. ${ }^{1} \mathrm{H}$ NMR (300 MHz, CDCl $\left.{ }_{3}\right) \delta 8.23\left(\mathrm{~d}, J=7.7 \mathrm{~Hz}, 2 \mathrm{H}, \mathrm{CH}_{\text {pyr }}\right)$, $8.16-8.00\left(\mathrm{~m}, 6 \mathrm{H}, \mathrm{CH}_{\text {pyr }}\right), 7.91\left(\mathrm{~d}, J=7.8 \mathrm{~Hz}, 1 \mathrm{H}, \mathrm{CH}_{\text {pyr }}\right), 7.47\left(\mathrm{~m}, 2 \mathrm{H}, \mathrm{CH}_{\text {arom }}\right), 7.25(\mathrm{~m}$, $\left.4 \mathrm{H}, \mathrm{CH}_{\text {arom }}\right), 6.51\left(\mathrm{~s}, 1 \mathrm{H}, \mathrm{CH}_{\text {imid }}\right), 5.76\left(\mathrm{~s}, 2 \mathrm{H}, \mathrm{CH}_{2}\right), 2.68-2.30\left(\mathrm{~m}, 4 \mathrm{H}, \mathrm{CH}_{i \mathrm{Pr}}\right)$, $1.40-1.03\left(\mathrm{~m}, 24 \mathrm{H}, \mathrm{CH}_{3, \mathrm{iPr}}\right)$. Electrospray MS (Cone $\left.20 \mathrm{~V}\right)(\mathrm{m} / \mathrm{z}$, fragment): 847.3 $[\mathrm{M}-\mathrm{OTf}+\mathrm{MeOH}]^{+}$. 


\section{$\underline{\text { SEM Images }}$}

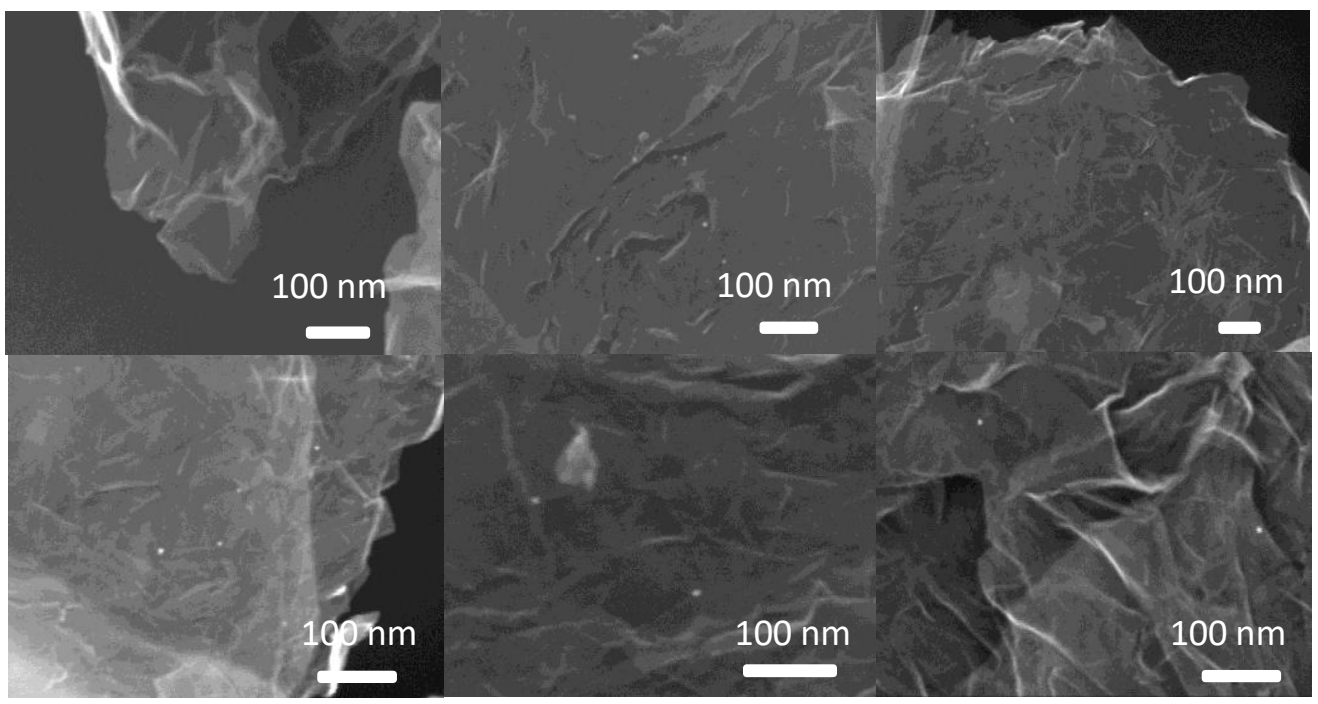

Figure 6.24 SEM images of 12I-rGO-NPs showing the presence of gold nanoparticles before catalysis

\section{$\underline{\text { TEM Images }}$}

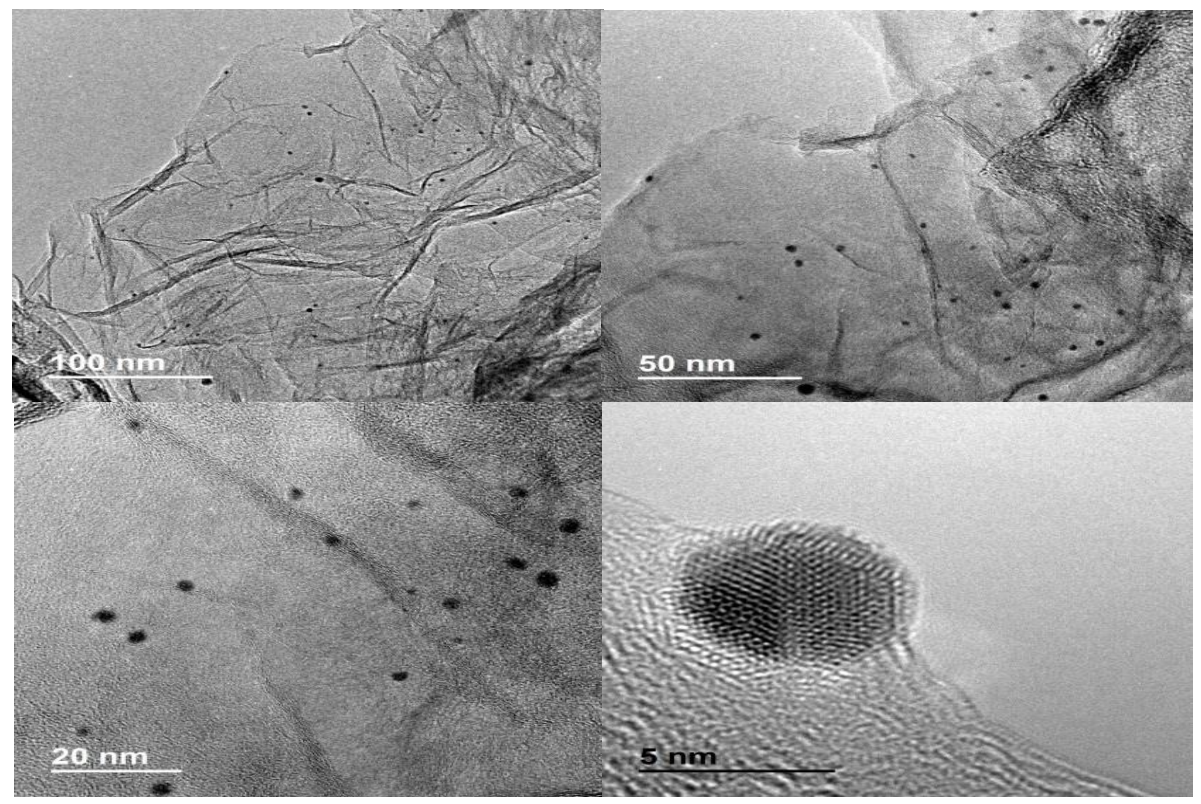

Figure 6.25 TEM images of 12I-rGO at different magnifications showing the presence of gold nanoparticles 


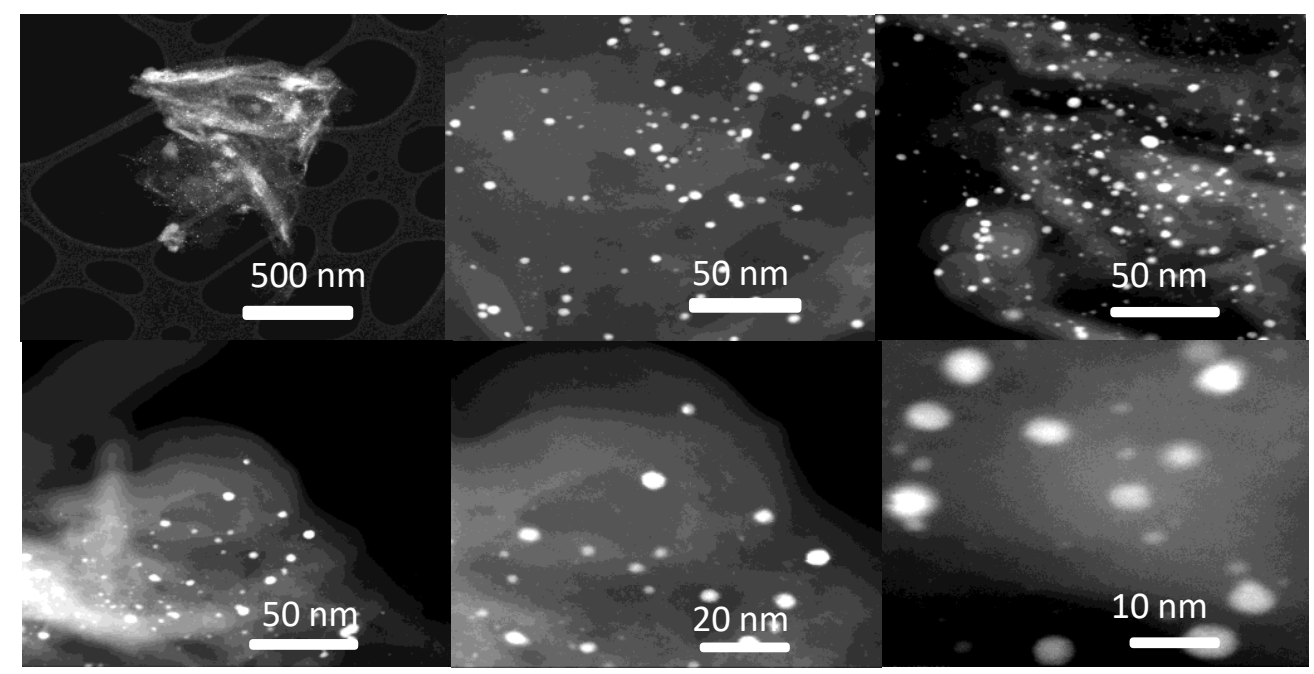

Figure 6.26 HAADF-STEM images of 12I-rGO-NPs showing the presence of gold nanoparticles

\section{$\underline{\text { UV/Vis spectroscopy }}$}

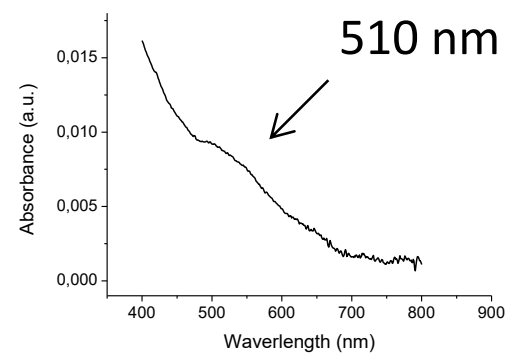

Figure 6.27 UV/Vis spectrum of 12I-rGO-NPs in water indicating the surface plasmon resonance of gold nanoparticles 


\section{XPS spectroscopy}
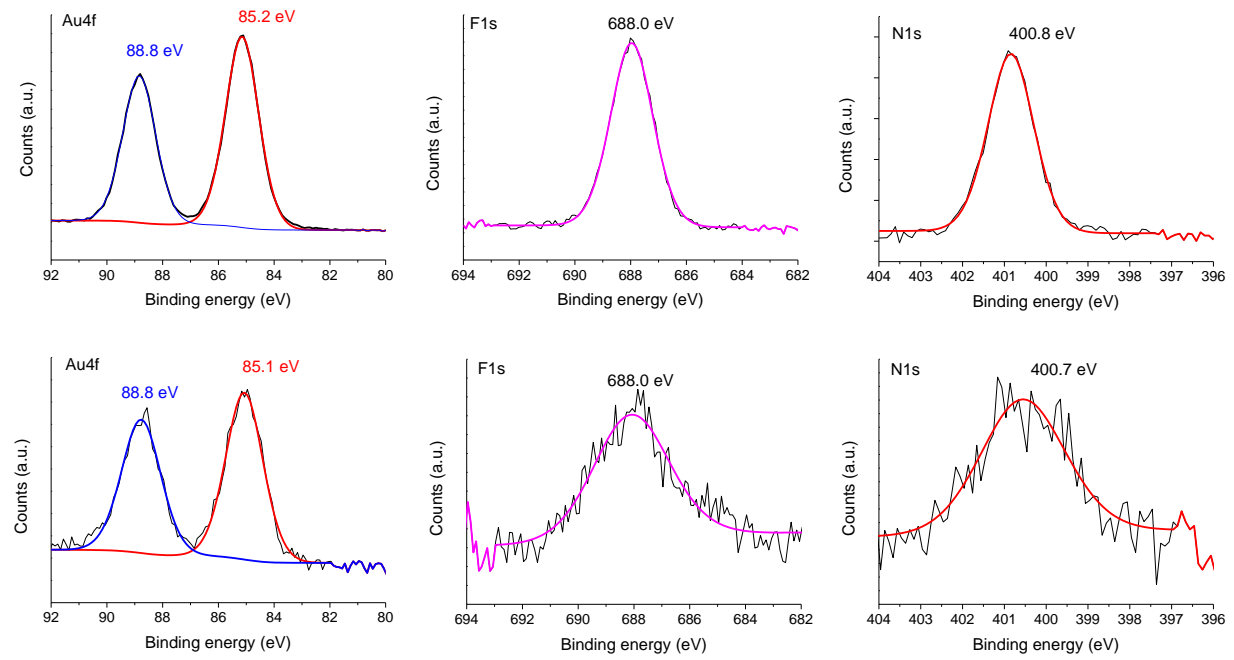

Figure 6.28 Comparative XPS analysis of molecular complex 12I (top) and hybrid material 12I-rGO-NPs (bottom) for the core-level peaks of Au4f, F1s and N1s

\section{Synthesis of $\mathrm{HAuCl}_{4}-\mathrm{rGO}-\mathrm{NPs}$}

A suspension of $\mathrm{rGO}(90 \mathrm{mg})$ in of $\mathrm{CH}_{2} \mathrm{Cl}_{2}(65 \mathrm{~mL})$ was sonicated for $30 \mathrm{~min}$. Then, $\mathrm{HAuCl}_{4} \cdot 3 \mathrm{H}_{2} \mathrm{O}(22,5 \mathrm{mg}, 0.06 \mathrm{mmol})$ was added to the suspension and was stirred at room temperature for 48 hours. The black solid was isolated by filtration and washed with $100 \mathrm{~mL}$ of $\mathrm{CH}_{2} \mathrm{Cl}_{2}$ affording the hybrid material $\mathrm{HAuCl}_{4}-\mathrm{rGO}-\mathbf{N P s}$. The exact amount of supported complex was determined by ICP-MS analysis. The results accounted for a $0.028 \mathrm{mg} \mathrm{Au} / 100 \mathrm{mg} \mathrm{rGO}$ of gold in the hybrid material $\mathrm{HAuCl}_{4}$-rGO-NPs. 


\section{$\underline{\text { TEM images }}$}

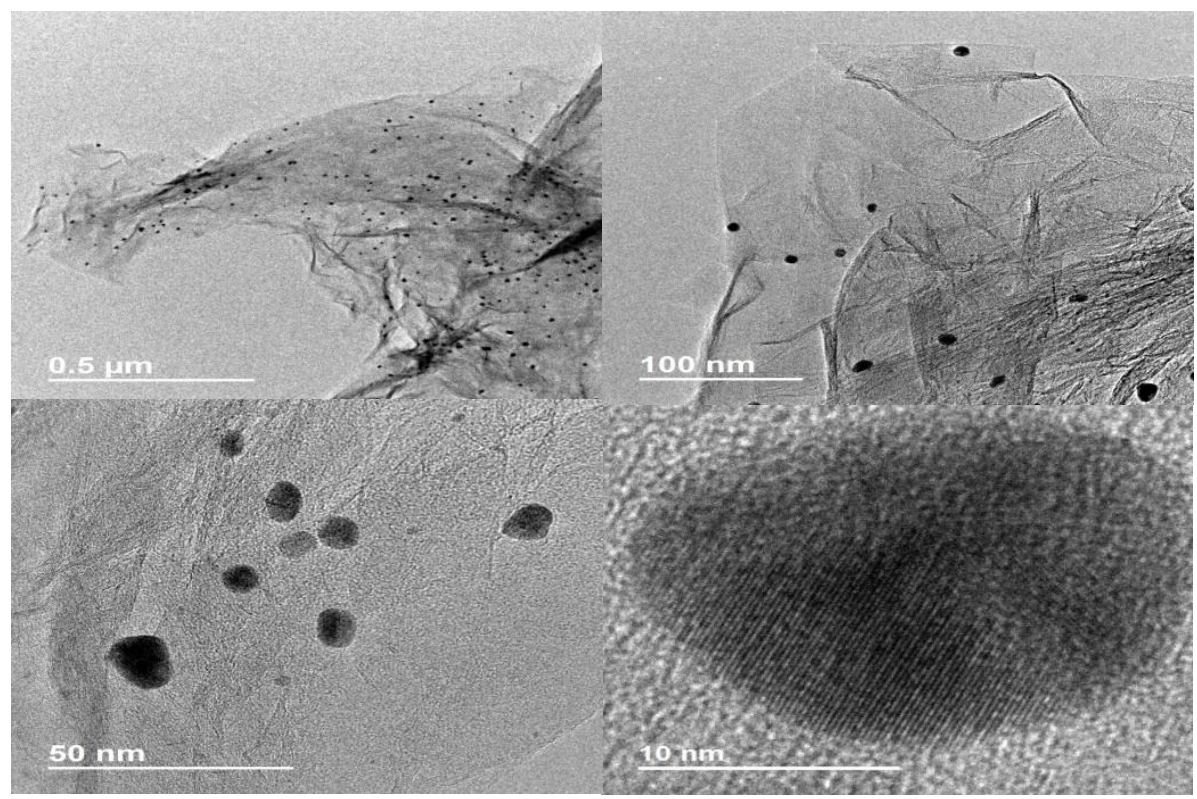

Figure 6.29 TEM images of $\mathrm{HAuCl}_{4}-\mathrm{rGO}-\mathrm{NPs}$ at different magnifications showing the presence of gold nanoparticles

\section{XPS spectroscopy}

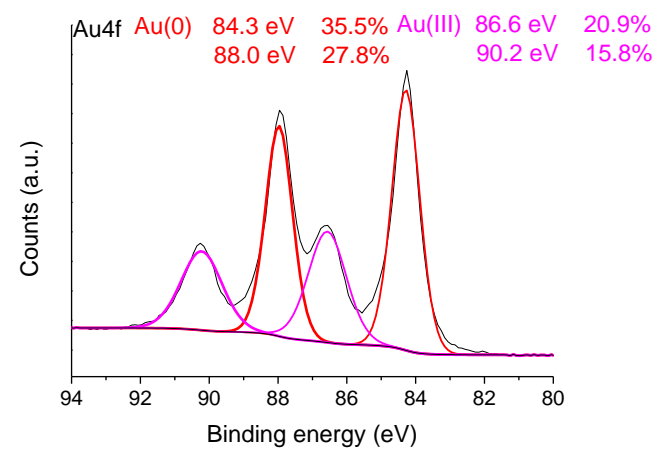

Figure 6.30 XPS spectra of $\mathrm{HAuCl}_{4}$-rGO-NPs for the Au $4 \mathrm{f}$ core-level peaks 


\subsection{CATALYTIC EXPERIMENTS}

\subsubsection{Dehydrogentative oxidation of alcohols}

Catalytic assays were performed in a round bottom flask using $0.5 \mathrm{mmol}$ of alcohol, 1 eq. of cesium carbonate, catalyst and $10 \mathrm{~mL}$ of Milli-Q water, and heating for 24 hours at $100{ }^{\circ} \mathrm{C}$. Conversions were determined by GC analysis using anisole as standard. After the reaction, the mixture was extracted with diethyl ether and the aqueous phase was acidified with $10 \mathrm{~mL}$ of $5 \mathrm{M} \mathrm{HCl}$ and extraxted with EtOAc. The organic phase was dried over $\mathrm{MgSO}_{4}$ and the solvent evaporated under reduced pressure affording the pure acid. Isolated yields were determined by ${ }^{1} \mathrm{H}$ NMR using trimethoxy benzene as the standard.

Recycling experiments: Recycling experiments were carried out under the same reaction conditions as described before. After completion of each run (24 h), the reaction mixture was allowed to reach room temperature and the catalyst was isolated by decantation. The remaining solid was washed thoroughly with water and reused in the following run. 
Time-conversion profile for the dehydrogenation of ( $p$-trifluoromethyl) benzyl alcohol

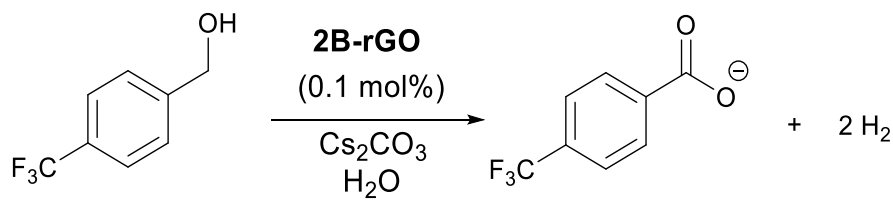

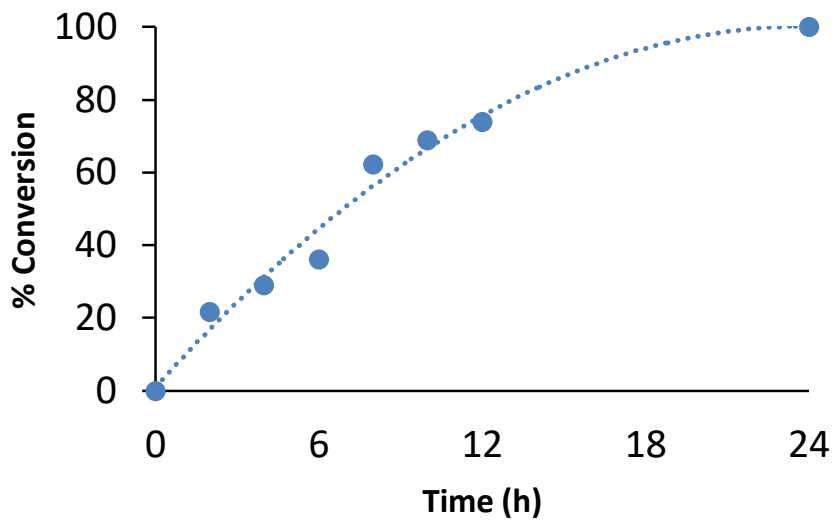

Figure 6.31 Time-conversion profile for the dehydrogenation of ( $p$-trifluoromethyl) benzyl alcohol. Reaction conditions: Substrate $(0.5 \mathrm{mmol}), \mathrm{Cs}_{2} \mathrm{CO}_{3}(0.5 \mathrm{mmol})$, catalyst (2B-rGO, $0.1 \mathrm{~mol} \%), 10 \mathrm{~mL}$ of water at $100{ }^{\circ} \mathrm{C}$. Conversions determined by $\mathrm{GC}$ using anisole as standard

\section{Procedure for hydrogen identification}

All glassware was carefully clean and rinsed with Milli-Q water prior to use. A $25 \mathrm{~mL}$ round bottom flask was charged with $0.44 \mathrm{mmol}$ of 4-methyl benzyl alcohol, $0.44 \mathrm{mmol}$ of cesium carbonate, $8.810^{-3} \mathrm{mmol}$ of catalyst (1A) and $10 \mathrm{~mL}$ of water and refluxed at $100^{\circ} \mathrm{C}$ in a closed system. At selected times (ca. $50 \%$ conversion), a $1.5 \mathrm{~mL}$ sample of the generated gas was collected using a gas syringe and the hydrogen content was qualitative analysed by gas chromatography (GS-MOL 15 meters column ID 0.55 mm TCD from J\&W Scientific).

\subsubsection{Dehydrogenation of amines}

Catalytic reactions were performed in a round bottom flask using 1 eq. of amine, $2 \mathrm{~mol} \%$ of catalyst, and $3 \mathrm{~mL}$ of toluene, and heating for a suitable period of time at $110^{\circ} \mathrm{C}$. The yields and conversions were determined by GC analysis using anisole as the internal standard. The isolated yields were evaluated by ${ }^{1} \mathrm{H} N M R$ integration using 1,3,5 trimethoxybenzene as the standard. 
Recycling experiments: Recycling experiments were carried out under the same reaction conditions as described before. After completion of each run $(2 \mathrm{~h}, \mathrm{ca} .50 \%$ conversion), the reaction mixture was allowed to reach room temperature and the catalyst was isolated by decantation. The remaining solid was washed thoroughly with toluene and reused in the following run.

\section{Time-conversion profile for the dehydrogenation of ( $p$-methoxy) benzyl amine}
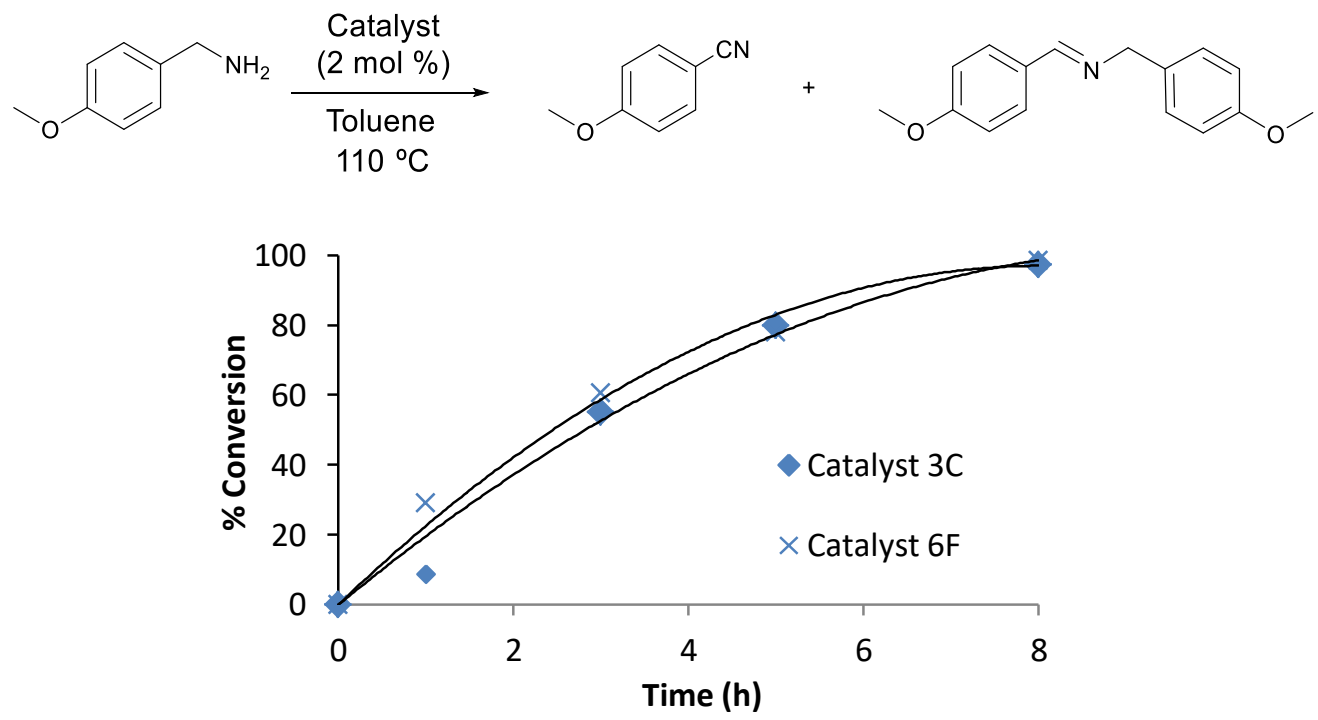

Figure 6.32 Time-conversion profile for the dehydrogenation of ( $p$-methoxy) benzyl amine. Reaction conditions: Substrate $(0.3 \mathrm{mmol})$, catalyst $(6 \mathrm{~F}, 2 \mathrm{~mol} \%), 2 \mathrm{~mL}$ of toluene at $110^{\circ} \mathrm{C}$. Conversions determined by GC using anisole as standard

\section{Procedure for hydrogen identification.}

All glassware was carefully cleaned with Milli-Q water and rinsed with acetone prior to use. A $25 \mathrm{~mL}$ round bottom flask was charged with $0.30 \mathrm{mmol}$ of amine, $2 \mathrm{~mol} \%$ of ruthenium catalyst (6F) and $2 \mathrm{~mL}$ of toluene and refluxed at $110{ }^{\circ} \mathrm{C}$ in a closed system. At selected times (ca. $50 \%$ conversion), a $1.5 \mathrm{~mL}$ sample of the generated gas was collected using a gas syringe and the hydrogen content was qualitative analysed by gas chromatography (GS-MOL 15 meters, column ID $0.55 \mathrm{~mm}$, TCD from J\&W Scientific).

\subsubsection{Dehydrogenative coupling between silanes and alcohols}

Catalytic experiments were performed in a $25 \mathrm{~mL}$ round bottom flask, using $0.5 \mathrm{mmol}$ of silane, $1 \mathrm{~mL}$ of alcohol, ruthenium $(3 \mathrm{C})$ or iridium $(8 \mathrm{C})$ catalyst 
$(0.05-1.00 \mathrm{~mol} \%)$ and heating at $30{ }^{\circ} \mathrm{C}$ under an initial aerobic atmosphere. Yields and conversions were determined by GC analysis using anisole as internal standard. Isolated yields were determined by solvent evaporation and analysis by ${ }^{1} \mathrm{H}$ NMR spectroscopy using 1,3,5-trimethoxybenzene as standard. For the determination of yields based on the evolution of gas the system was connected to an inverted burette filled with water that was used to collect the gas released and monitor the reaction or a pressure transducer was used (see Figure 6.33).

Inverted Burette

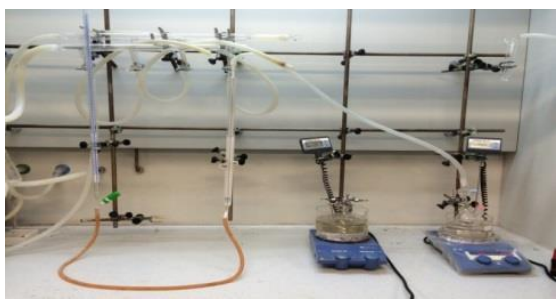

Pressure Transducer

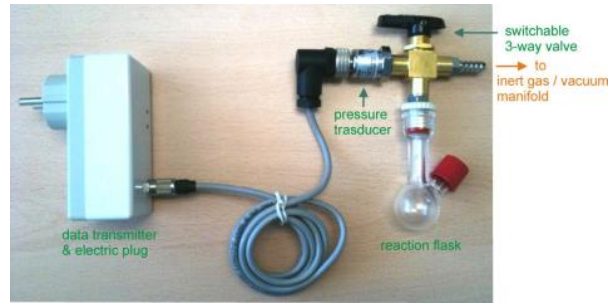

Figure 6.33 Systems used for the measurement of the evolved hydrogen

Recycling experiments: Recycling experiments were performed in a Pyrex ${ }^{\circledR}$ tube open to the atmosphere. The tube was charged with $0,5 \mathrm{mmol}$ of dimethylphenyl silane, $1 \mathrm{~mL}$ of methanol, and $0.5 \mathrm{~mol} \%$ of ruthenium or iridium catalyst (3C-rGO or $\mathbf{8 C}$-rGO respectively) stirring at $30^{\circ} \mathrm{C}$ for $10 \mathrm{~min}$. Conversions and yields were determined by GC. After each run the reaction mixture was centrifuged and the solid catalyst was washed 3 times with $2 \mathrm{~mL}$ dichloromethane and 1 time with $2 \mathrm{~mL}$ pentane and dried before using it in the following run.

Large Scale experiment: Large-scale catalytic experiment was performed under the same conditions described in the general procedure. A $100 \mathrm{~mL}$ round bottom flask was charged with $68 \mathrm{mmol}$ of dimethylphenyl silane $(10 \mathrm{~g}) 50 \mathrm{~mL}$ of alcohol and $40 \mathrm{mg}$ of Ru catalyst $3 \mathrm{C}(0.068 \mathrm{mmol}, 0.1 \mathrm{~mol} \%)$. After completion of reaction (30 $\mathrm{min})$, the solvent was removed by evaporation and the yield determined by gravimetric analysis. 
Reaction profile for the dehydrogenative coupling betweet methanol and dimethyl-phenyl-silane catalysed by $3 \mathrm{C}$ or $8 \mathrm{C}$
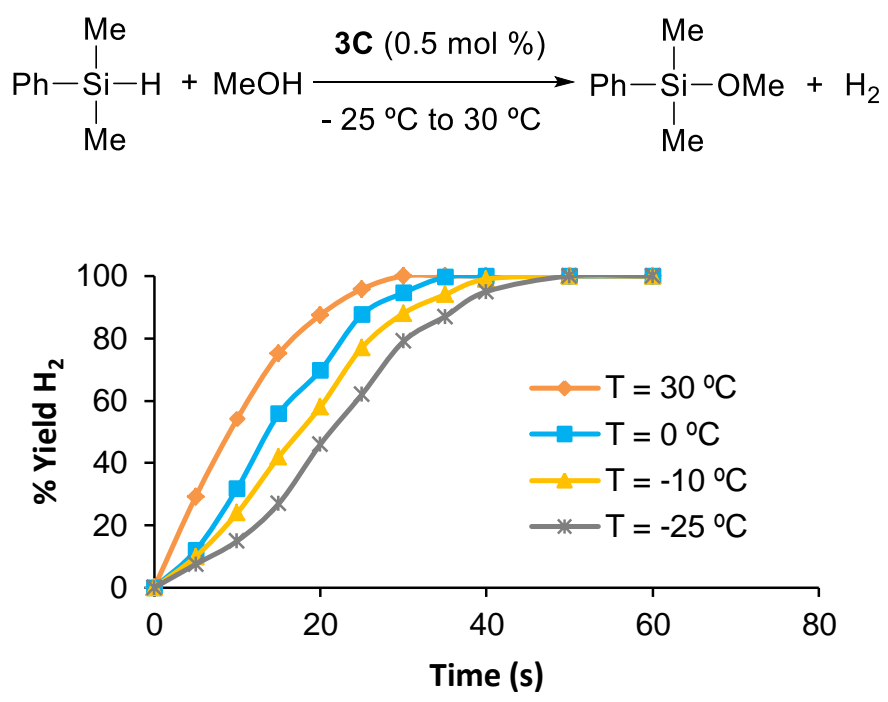

Figure 6.34 Reaction profile at different temperatures. Reaction conditions: dimethylphenyl silane $(1.0 \mathrm{mmol})$, catalyst (3C, $0.5 \mathrm{~mol} \%), 1 \mathrm{~mL}$ of $\mathrm{MeOH}$ at the selected temperature. Yield corresponds to the amount of hydrogen collected using the inverted burette system depicted in Figure 6.33

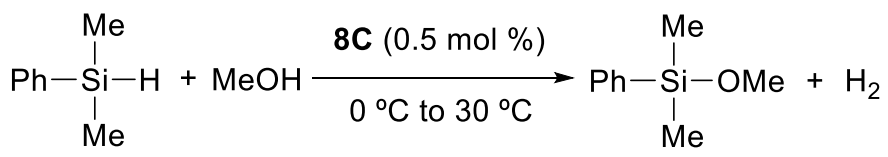

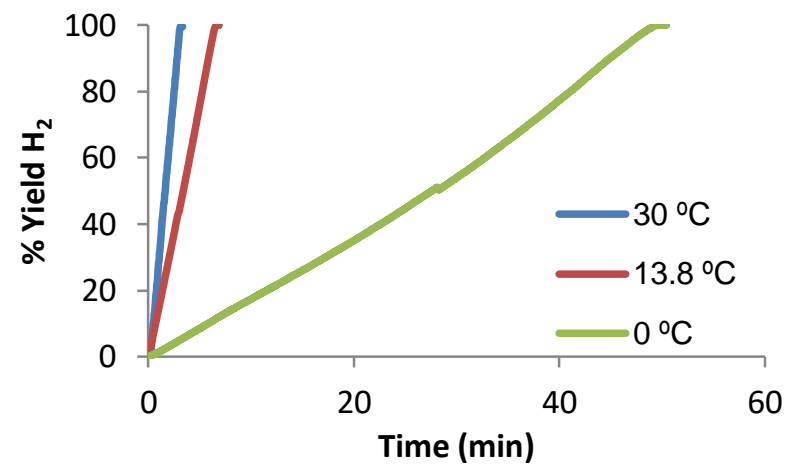

Figure 6.35 Reaction profile at different temperatures. Reaction conditions: dimethylphenylsilane $(1.0 \mathrm{mmol})$, catalyst $(8 \mathrm{C}, 0.5 \mathrm{~mol} \%), 1 \mathrm{~mL}$ of $\mathrm{MeOH}$ at the selected temperature. Yield corresponds to the amount of hydrogen collected using the pressure transducer system depicted in Figure 6.33 


\section{Procedure for hydrogen identification.}

All glassware was carefully cleaned and rinsed with Milli-Q water prior to use. A $25 \mathrm{~mL}$ two-necked round bottom flask was charged with $1 \mathrm{~mL}$ of methanol and $1 \mathrm{~mol} \%$ of catalyst $\mathbf{3 C}$ or $\mathbf{8 C}$ and stirred at room temperature for $5 \mathrm{~min}$. Dimethylphenyl silane $(1 \mathrm{mmol})$ was added with the aid of a syringe through a septum rubber. After 10 seconds, the gas evolved was captured with a syringe and injected in a quadrupole mass spectrometer equipment (Omnistar GSD 32003 from PFEIFFER VACUUM) confirming the presence of molecular hydrogen.

\section{Stability of catalysts 3C and 8C: Sequential methanolysis of dimethylphenylsilane}
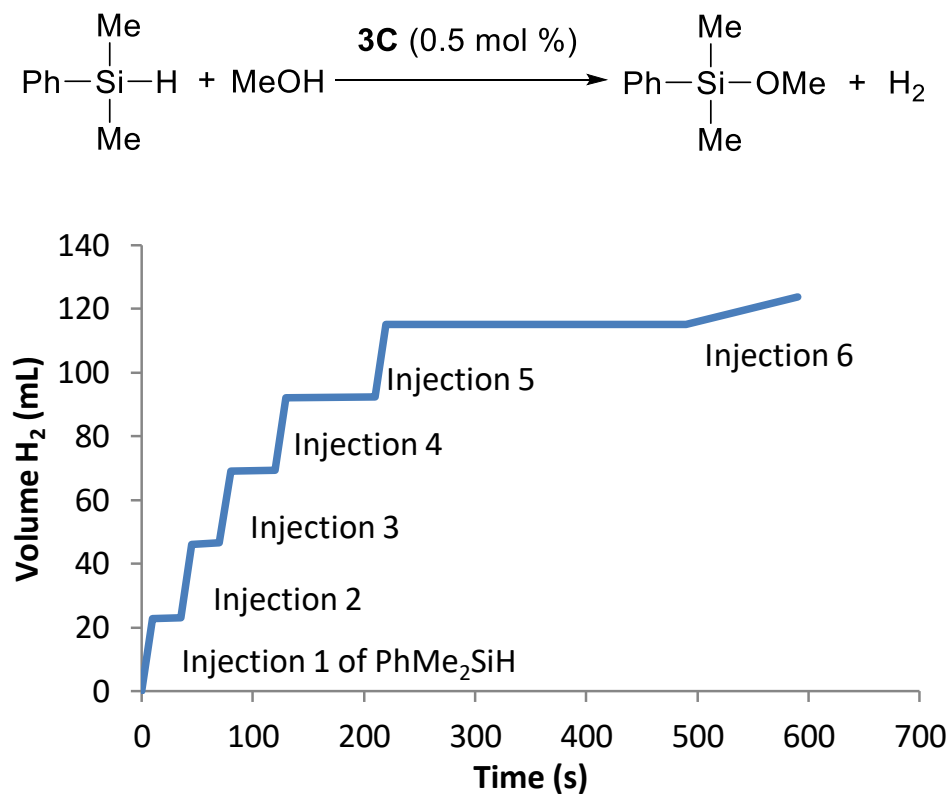

Figure 6.36 Time-profile for the reaction between dimethylphenyl silane and methanol catalysed by $\mathbf{3 C}$ obtained over six additions of silane. Measurements done using the inverted burette system depicted in Figure 6.33 An evolved volume of $24 \mathrm{~mL}$ corresponds to $100 \%$ yield. From injections 1 to 4 quantitative yields were obtained. In the 5 th injection the yield drops to $75 \%$ and in the last injection the yield decreases to $36 \%$ 


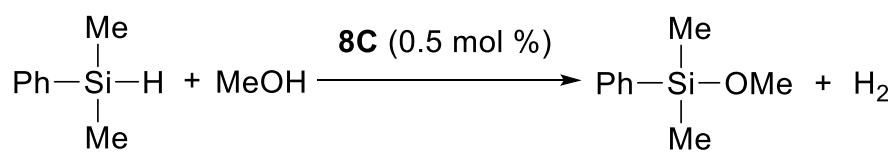

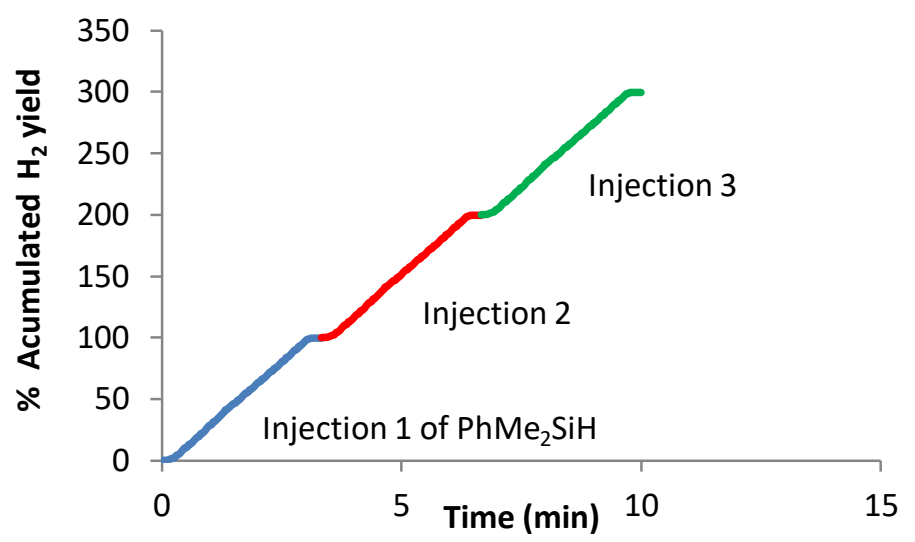

Figure 6.37 Time-profile for the reaction between dimethylphenyl silane and methanol catalysed by $\mathbf{8 C}$ obtained over three additions of silane. Measurements done using the pressure transducer system depicted in Figure 6.33

\subsubsection{Intramolecular hydroamination}

Catalytic experiments were performed under air in a pyrex tube ${ }^{\circledR}$ using $0.2 \mathrm{mmol}$ of substrate, $2.4 \mathrm{~mL}$ of solvent, catalyst (0.1 - $2.0 \mathrm{~mol} \%$ ) and $\mathrm{AgBF}_{4}$ (6 mol \%), and heating at $50{ }^{\circ} \mathrm{C}$. Yields and conversions were determined by GC analysis using anisole as internal standard. Isolated yields were determined by ${ }^{1} \mathrm{H}$ NMR spectroscopy using 1,3,5-trimethoxybenzene as standard.

Recycling experiments: Recycling experiments were carried out under the same reaction conditions as described before. After completion of each run the reaction mixture was allowed to reach room temperature and the catalyst was isolated by decantation. The remaining solid was washed thoroughly with toluene and reused in the following run. 
Time-conversion profile for the intramolecular hydroamination of 2(phenylethynyl) aniline
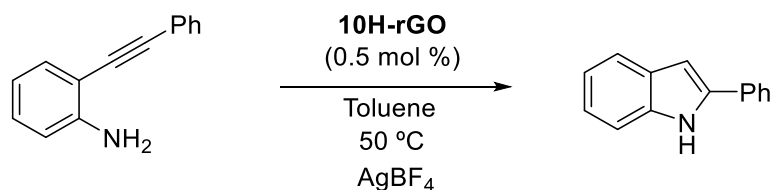

$\mathrm{AgBF}_{4}$

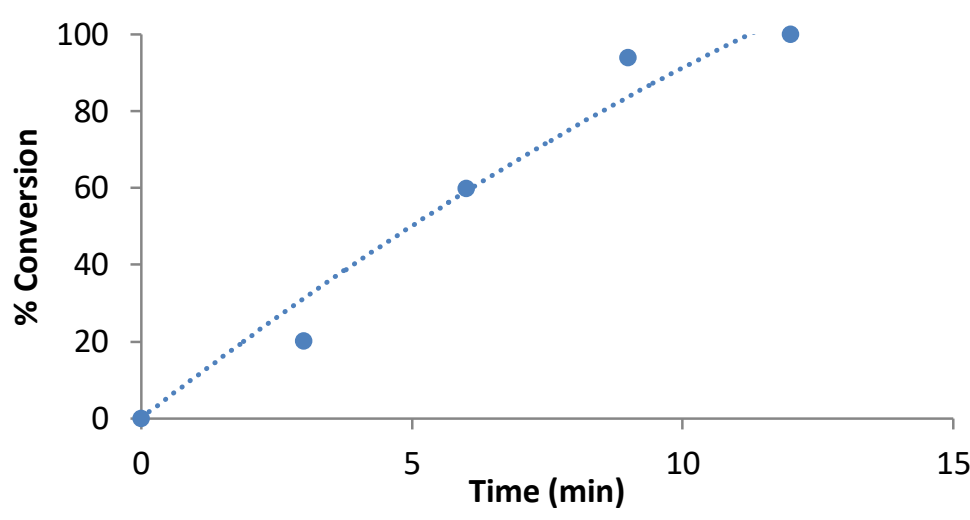

Figure 6.38 Time-conversion profile for the intramolecular hydroamination of 2(phenylethynyl) aniline. Reaction conditions: Substrate $(0.2 \mathrm{mmol})$, catalyst (10H-rGO, $0.5 \mathrm{~mol} \%), 2.4 \mathrm{~mL}$ of toluene at $50{ }^{\circ} \mathrm{C}$. Conversion determined by $\mathrm{GC}$ using anisole as standard

\subsubsection{Hydration of alkynes}

In a Pyrex ${ }^{\circledR}$ tube and under air, the corresponding alkyne (1 eq.) water (2 eq.), catalyst (12l or 12I-rGO-NPs), and methanol (concentration of alkyne $0.15 \mathrm{M}$ ) were mixed. The reaction was stirred at $50^{\circ} \mathrm{C}$ in an oil bath. Conversion of alkyne into the corresponding ketone was monitored by GC-FID using anisole as an internal standard. When the reaction was completed, the solvent was removed and the yield of isolated product analysed by NMR.

Recycling experiments: Recycling experiments were carried out under the same reaction conditions as described before. After completion of each run the reaction mixture was allowed to reach room temperature and the catalyst was isolated by decantation. The remaining solid was washed thoroughly with pentane and reused in the following run. 
Time-conversion profile for the catalytic hydration of 4-octyne
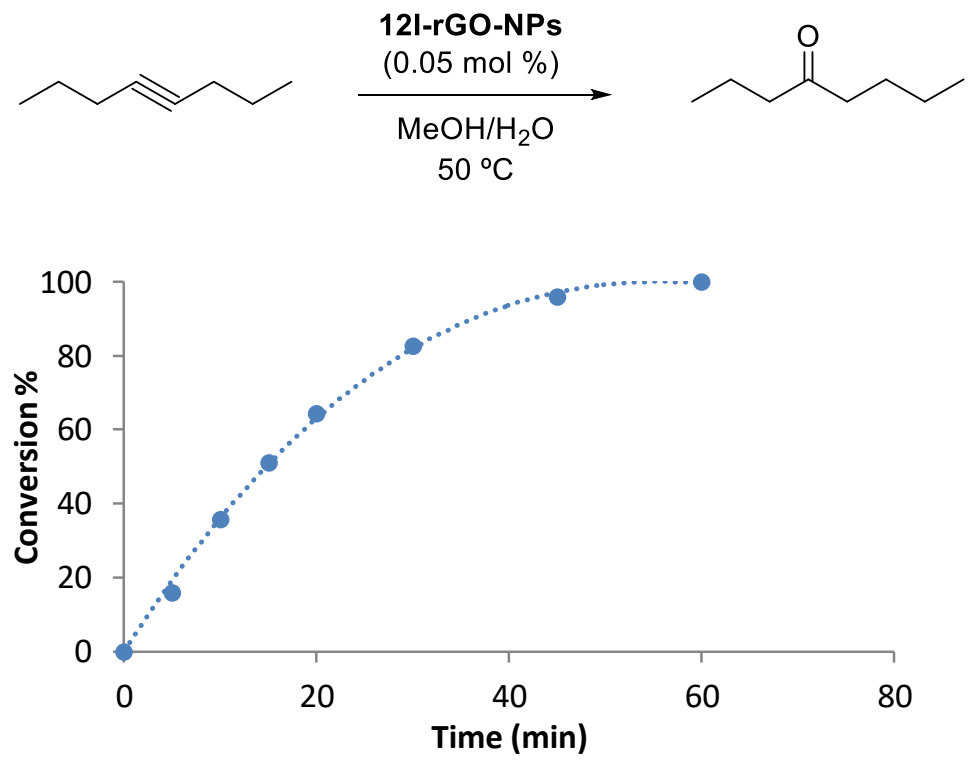

Figure 6.39 Time-conversion profile for the catalytic hydration of 4-octyne. Reaction conditions: Substrate $(0.2 \mathrm{mmol}$ ), catalyst (12I-rGO-NPs, $0.05 \mathrm{~mol} \%) 1.4 \mathrm{~mL}$ of $\mathrm{MeOH}, 7.2 \mu \mathrm{l}$ $\mathrm{H}_{2} \mathrm{O}$ (0.4 mmol, 2eq.) at $50{ }^{\circ} \mathrm{C}$. Conversion determined by GC using anisole as standard

\subsection{DFT STUDIES}

\section{General considerations}

Quantum mechanical calculations were performed with the Gaussian09 ${ }^{[16]}$ package at the DFT/M06 level of theory. ${ }^{[17]}$ SDD basis set and its corresponding effective core potentials (ECPs) were used to describe the ruthenium or iridium atom. ${ }^{[18]} \mathrm{An}$ additional set of $\mathrm{f}$-type functions was also added. ${ }^{[19]}$ Carbon, nitrogen, oxygen, silicon and hydrogen atoms were described with a 6-31G** basis set. $^{[20,21]}$

\section{Ruthenium catalysed dehydrogenative oxidation of alcohols in water media}

Frequency calculations have been performed in order to determine the nature of the stationary points found (no imaginary frequencies for minima, only one imaginary frequency for transition states). Free Gibbs energies in water solution ( $\varepsilon=78.3553$ ) were calculated by computing the energy in the solvent by means of single-point calculations on the gas-phase optimized geometries with the SMD continuum solvation model, ${ }^{[22]}$ and subsequently applying the following expression: 
$G_{\text {water }}=E_{\text {water }}+\left(G_{\text {gas phase }}-E_{\text {gas phase }}\right)$

Dehydrogenative coupling between silanes and alcohols

All calculations were performed in methanol solution $(\varepsilon=32.613$ ) by means of the SMD continuum solvation model. ${ }^{[22]}$ The structures were freely optimized with no symmetry restrictions. Frequency calculations were subsequently performed in order to determine the nature of the stationary points found. No transition structures for the $S_{N}{ }^{2}$ processes could be located, mainly because of the virtually barrierless nature of these processes. Instead they were carefully studied by using the following freeze-scan strategy: Si $\cdots O$ distances were frozen in subsequent $0.05 \AA$ steps, and the structures were optimized with this geometrical restriction. Furthermore, this strategy was repeated by using $0.01 \AA$ steps in the Si-O distance range from 2.00 to $1.85 \AA$, the critical range in which simultaneously the $\mathrm{Si}-\mathrm{O}$ bond is forming, the $\mathrm{Si}-\mathrm{H}$ bond is breaking and the $\mathrm{Ru}-\mathrm{H}$ (or $\mathrm{Ir}-\mathrm{H}$ ) bond is forming. Such analysis afforded energy curves for the reaction coordinate connecting minima II and III (Scheme 4.2) and an estimate of the transition state structure, with its corresponding electronic energy (ETS*). The activation free energy was estimated by applying the expression $\mathrm{G} \ddagger^{*}=\mathrm{G}(\mathrm{II})+\left(\mathrm{ETS}^{*}-\mathrm{E}(\mathrm{II})\right)$.

\subsection{X-RAY DIFFRACTION}

Single crystals suitable for X-ray diffraction were mainly obtained by slow diffusion of hexane or diethyl ether into a concentrated solution of the compound in different solvents or by slow evaporation of a concentrated solution of the compound in appropriated solvent. Diffraction data was collected on a Agilent SuperNova diffractometer equipped with an Atlas CCD detector using Mo-Ka radiation $\left(\lambda=0.71073 \AA\right.$ ) . Single crystals were mounted on a MicroMount ${ }^{\circledR}$ polymer tip (MiteGen) in a random orientation. Absorption corrections based on the multiscan method were applied. ${ }^{[23]}$ Structures were solved by direct methods in SHELXS-97 and refined by the full-matrix method based on $\mathrm{F}^{2}$ with the program SHELXL-97 using the OLEX software package. ${ }^{[24,25]}$

The following tables summarise key details of the crystal and structure refinement data. 
Table 6.1 Structural parameters, register conditions and refinement of $\mathbf{3 A}, \mathbf{3 C}$ and 7G'

\begin{tabular}{|c|c|c|c|}
\hline & $3 A$ & $3 C$ & 7G' \\
\hline Empirical formula & $\mathrm{C}_{22} \mathrm{H}_{22} \mathrm{Cl}_{5} \mathrm{~F}_{5} \mathrm{~N}_{2} \mathrm{Ru}$ & $\mathrm{C}_{36} \mathrm{H}_{39} \mathrm{Cl}_{8} \mathrm{~N}_{2} \mathrm{Ru}$ & $\mathrm{C}_{23} \mathrm{H}_{33} \mathrm{BClF}_{4} \mathrm{~N}_{3} \mathrm{Ru}$ \\
\hline Formula weight & 687.74 & 884.36 & 574.85 \\
\hline Temperature/K & $293(2)$ & $293(2)$ & $200.00(14)$ \\
\hline Crystal system & monoclinic & triclinic & orthorhombic \\
\hline Space group & $\mathrm{P} 21 / \mathrm{c}$ & P-1 & $\mathrm{Pna}_{1}$ \\
\hline $\mathrm{a} / \AA ̊$ & $15.9690(4)$ & $11.3681(4)$ & $13.5393(8)$ \\
\hline $\mathrm{b} / \AA$ & $10.6225(4)$ & $14.3155(7)$ & $16.4138(11)$ \\
\hline$c / \AA$ & $15.6395(5)$ & $14.8357(7)$ & $11.4752(8)$ \\
\hline$\alpha /^{\circ}$ & 90.00 & $62.367(5)$ & 90 \\
\hline$\beta /{ }^{\circ}$ & $91.922(3)$ & $84.064(4)$ & 90 \\
\hline$\gamma /{ }^{\circ}$ & 90.00 & $77.725(4)$ & 90 \\
\hline Volume $/ \AA^{3}$ & $2651.44(15)$ & $2090.06(17)$ & $2550.1(3)$ \\
\hline Z & 4 & 2 & 4 \\
\hline$\rho_{\text {calc }} \mathrm{g} / \mathrm{cm}^{3}$ & 1.723 & 1.405 & 1.497 \\
\hline$\mu / \mathrm{mm}^{-1}$ & 1.146 & 0.913 & 0.765 \\
\hline$F(000)$ & 1368.0 & 898.0 & 1176.0 \\
\hline Crystal size $/ \mathrm{mm}^{3}$ & $0.11 \times 0.1 \times 0.09$ & $0.13 \times 0.1 \times 0.09$ & $0.19 \times 0.1 \times 0.06$ \\
\hline $2 \Theta$ range for data collection ${ }^{\circ}$ & 5.72 to 58.92 & 5.82 to 59.06 & 5.804 to 51.98 \\
\hline Index ranges & $\begin{array}{c}-22 \leq h \leq 22 \\
-14 \leq k \leq 13 \\
-21 \leq I \leq 20\end{array}$ & $\begin{array}{c}-15 \leq h \leq 15 \\
-19 \leq k \leq 19 \\
-20 \leq l \leq 20\end{array}$ & $\begin{array}{c}-16 \leq h \leq 13 \\
-20 \leq k \leq 15 \\
-13 \leq \mid \leq 14\end{array}$ \\
\hline Reflections collected & 29917 & 45813 & 13725 \\
\hline Independent reflections & $\begin{array}{c}6622 \\
{\left[R_{\text {int }}=0.0518,\right.} \\
\left.R_{\text {sigma }}=0.0391\right]\end{array}$ & $\begin{array}{c}10614 \\
{\left[R_{\text {int }}=0.0570,\right.} \\
\left.R_{\text {sigma }}=0.0449\right]\end{array}$ & $\begin{array}{c}4498 \\
{\left[R_{\text {int }}=0.0679\right.} \\
\left.R_{\text {sigma }}=0.0628\right]\end{array}$ \\
\hline Data/restraints/parameters & $6622 / 0 / 347$ & $10614 / 81 / 491$ & $4498 / 31 / 349$ \\
\hline Goodness-of-fit on $F^{2}$ & 0.859 & 1.069 & 1.071 \\
\hline Final $R$ indexes $[\mid>=2 \sigma(I)]$ & $\begin{array}{c}R_{1}=0.0351, \\
w R_{2}=0.0833\end{array}$ & $\begin{array}{c}\mathrm{R}_{1}=0.0613 \\
\mathrm{WR}_{2}=0.1725\end{array}$ & $\begin{array}{c}R_{1}=0.0448 \\
W R_{2}=0.1016\end{array}$ \\
\hline Final $R$ indexes [all data] & $\begin{array}{c}R_{1}=0.0463 \\
w R_{2}=0.0997\end{array}$ & $\begin{array}{c}R_{1}=0.0766 \\
w R_{2}=0.1917\end{array}$ & $\begin{array}{c}R_{1}=0.0553 \\
W R_{2}=0.1140\end{array}$ \\
\hline Largest diff. peak/hole / e $\AA^{-3}$ & $0.56 /-1.02$ & $1.80 /-0.98$ & $1.80 /-0.98$ \\
\hline
\end{tabular}


Table 6.2 Structural parameters, register conditions and refinement of $\mathbf{8 C}$ and $\mathbf{8 C ^ { \prime }}$

\begin{tabular}{|c|c|c|}
\hline & $8 C$ & $8 C^{\prime}$ \\
\hline Empirical formula & $\mathrm{C}_{31} \mathrm{H}_{31} \mathrm{Cl}_{2} \mathrm{IrN} \mathrm{N}_{2}$ & $\mathrm{C}_{31} \mathrm{H}_{30} \mathrm{BrIrN}$ \\
\hline Formula weight & 694.68 & 702.68 \\
\hline Temperature/K & $200.0(3)$ & $200.05(10)$ \\
\hline Crystal system & monoclinic & triclinic \\
\hline Space group & $\mathrm{P} 2{ }_{1} / \mathrm{n}$ & $P-1$ \\
\hline $\mathrm{a} / \AA ̊$ & $15.1236(8)$ & $8.67061(15)$ \\
\hline $\mathrm{b} / \AA$ & $10.0969(5)$ & $9.3192(2)$ \\
\hline$c / \AA$ & $17.7541(9)$ & $16.6791(5)$ \\
\hline$\alpha /^{\circ}$ & 90 & $103.488(2)$ \\
\hline$\beta /^{\circ}$ & $107.015(5)$ & $100.8497(19)$ \\
\hline$\gamma /{ }^{\circ}$ & 90 & $96.9463(17)$ \\
\hline Volume $/ \AA^{3}$ & $2592.4(2)$ & $1267.80(6)$ \\
\hline Z & 4 & 2 \\
\hline$\rho_{\text {calc }} \mathrm{g} / \mathrm{cm}^{3}$ & 1.780 & 1.841 \\
\hline$\mu / \mathrm{mm}^{-1}$ & 5.380 & 6.865 \\
\hline$F(000)$ & 1368.0 & 684.0 \\
\hline Crystal size $/ \mathrm{mm}^{3}$ & $0.11 \times 0.1 \times 0.04$ & $0.13 \times 0.11 \times 0.11$ \\
\hline $2 \Theta$ range for data collection $/^{\circ}$ & 4.798 to 51.994 & 5.8 to 58.742 \\
\hline Index ranges & $\begin{array}{c}-18 \leq h \leq 18 \\
-12 \leq k \leq 12 \\
-21 \leq l \leq 21\end{array}$ & $\begin{array}{l}-11 \leq \mathrm{h} \leq 11 \\
-12 \leq \mathrm{k} \leq 12 \\
-22 \leq \mathrm{I} \leq 22\end{array}$ \\
\hline Reflections collected & 26704 & 28120 \\
\hline Independent reflections & $\begin{array}{c}5105 \\
{\left[R_{\text {int }}=0.0439,\right.} \\
\left.R_{\text {sigma }}=0.0318\right]\end{array}$ & $\begin{array}{c}6403 \\
{\left[R_{\text {int }}=0.0331\right.} \\
\left.R_{\text {sigma }}=0.0312\right]\end{array}$ \\
\hline Data/restraints/parameters & $5105 / 0 / 331$ & $6403 / 0 / 322$ \\
\hline Goodness-of-fit on $\mathrm{F}^{2}$ & 1.057 & 1.061 \\
\hline Final $R$ indexes $[I>=2 \sigma(I)]$ & $\begin{array}{c}R_{1}=0.0261 \\
w R_{2}=0.0621\end{array}$ & $\begin{array}{c}R_{1}=0.0212 \\
w R_{2}=0.0411\end{array}$ \\
\hline Final $R$ indexes [all data] & $\begin{array}{c}R_{1}=0.0329 \\
w R_{2}=0.0665\end{array}$ & $\begin{array}{c}R_{1}=0.0263 \\
w R_{2}=0.0434\end{array}$ \\
\hline Largest diff. peak/hole / e $\AA^{-3}$ & $1.19 /-0.66$ & $1.00 /-0.73$ \\
\hline
\end{tabular}

Note: Suitable single crystals of $\mathbf{8} \mathbf{C}^{\prime}$ were obtained after halide exchange using $\mathrm{NaBr}$. 
Table 6.3 Structural parameters, register conditions and refinement of $\mathbf{1 0 H}$ and $\mathbf{1 1 I}$

\begin{tabular}{|c|c|c|}
\hline & $10 \mathrm{H}$ & 111 \\
\hline Empirical formula & $\mathrm{C}_{21} \mathrm{H}_{16} \mathrm{AuClN}_{2}$ & $\mathrm{C}_{44} \mathrm{H}_{46} \mathrm{AuBrN}{ }_{2} \mathrm{O}$ \\
\hline Formula weight & 528.77 & 895.70 \\
\hline Temperature/K & $200.00(10)$ & $293(2)$ \\
\hline Crystal system & monoclinic & monoclinic \\
\hline Space group & $\mathrm{P} 2_{1} / \mathrm{c}$ & $\mathrm{P} 2_{1} / \mathrm{n}$ \\
\hline $\mathrm{a} / \AA ̊$ & $12.2750(3)$ & $12.6295(7)$ \\
\hline $\mathrm{b} / \AA$ & $11.5743(3)$ & $23.6385(17)$ \\
\hline$c / \AA$ & $12.3319(3)$ & $13.2570(8)$ \\
\hline$\alpha /^{\circ}$ & 90 & 90 \\
\hline$\beta /^{\circ}$ & $96.802(2)$ & $94.723(5)$ \\
\hline$\gamma /{ }^{\circ}$ & 90 & 90 \\
\hline Volume $/ \AA^{3}$ & $1739.71(7)$ & $3944.3(4)$ \\
\hline Z & 4 & 4 \\
\hline$\rho_{\text {calc }} \mathrm{g} / \mathrm{cm}^{3}$ & 2.019 & 1.508 \\
\hline$\mu / \mathrm{mm}^{-1}$ & 8.615 & 4.777 \\
\hline$F(000)$ & 1008.0 & 1784.0 \\
\hline Crystal size $/ \mathrm{mm}^{3}$ & $0.12 \times 0.1 \times 0.09$ & $0.13 \times 0.10 \times 0.08$ \\
\hline $2 \Theta$ range for data collection $/^{\circ}$ & 5.656 to 51.998 & 4.728 to 59.236 \\
\hline Index ranges & $\begin{array}{c}-15 \leq h \leq 15 \\
-14 \leq k \leq 14 \\
-15 \leq l \leq 15\end{array}$ & $\begin{array}{c}-15 \leq h \leq 17 \\
-21 \leq k \leq 31 \\
-14 \leq I \leq 18\end{array}$ \\
\hline Reflections collected & 33714 & 25843 \\
\hline Independent reflections & $\begin{array}{c}3420 \\
{\left[R_{\text {int }}=0.0338,\right.} \\
\left.R_{\text {sigma }}=0.0160\right]\end{array}$ & $\begin{array}{c}9599 \\
{\left[R_{\text {int }}=0.0425\right.} \\
\left.R_{\text {sigma }}=0.0593\right]\end{array}$ \\
\hline Data/restraints/parameters & $3420 / 0 / 275$ & $9599 / 0 / 450$ \\
\hline Goodness-of-fit on $\mathrm{F}^{2}$ & 1.066 & 1.027 \\
\hline Final $R$ indexes $[I>=2 \sigma(I)]$ & $\begin{array}{c}R_{1}=0.0210 \\
w R_{2}=0.0563\end{array}$ & $\begin{array}{c}R_{1}=0.0473 \\
W R_{2}=0.0819\end{array}$ \\
\hline Final $R$ indexes [all data] & $\begin{array}{l}R_{1}=0.0253 \\
w R_{2}=0.0590\end{array}$ & $\begin{array}{c}R_{1}=0.0824 \\
W R_{2}=0.0974\end{array}$ \\
\hline Largest diff. peak/hole / e $\AA^{-3}$ & $1.09 /-0.42$ & $1.53 /-1.43$ \\
\hline
\end{tabular}




\subsection{REFERENCES}

[1] R. Uson, A. Laguna, M. Laguna, D. A. Briggs, H. H. Murray, J. P. Fackler, Inorganic. Synth. 1989, 26, 85-91.

[2] Y. He, M. F. Lv, C. Cai, Dalton Trans. 2012, 41, 12428-12433.

[3] J. L. Bartels, P. Lu, A. Walker, K. Maurer, K. D. Moeller, Chem. Commun. 2009, $37,5573-5575$.

[4] L. Benhamou, V. César, H. Gornitzka, N. Lugan, G. Lavigne, Chem. Commun. 2009, 31, 4720-4722.

[5] J. P. Brand, C. Chevalley, J. Waser, Beilstein J. Org. Chem. 2011, 7, 565-569.

[6] S. McGrandle, G. C. Saunders, J. Fluor. Chem. 2005, 126, 449-453.

[7] Z. Fei, D. R. Zhu, X. Yang, L. Meng, Q. Lu, W. H. Ang, R. Scopelliti, C. G. Hartinger, P. J. Dyson, Chem. - A Eur. J. 2010, 16, 6473-6481.

[8] S. Sabater, J. A. Mata, E. Peris, ACS Catal. 2014, 4, 2038-2047.

[9] I. Dinarès, C. Garcia De Miguel, A. Ibáñez, N. Mesquida, E. Alcalde, Green Chem. 2009, 11, 1507-1510.

[10] A. J. Arduengo, III. Preparation of 1,3-Disubstituted Imidazolium Salts, 1993, U.S. Patent 5182405.

[11] R. Corberán, M. Sanaú, E. Peris, J. Am. Chem. Soc. 2006, 128, 3974-3979.

[12] S. Gardner, T. Kawamoto, D. P. Curran, J. Org. Chem. 2015, 80, 9794-9797.

[13] L. Mercs, A. Neels, M. Albrecht, J. Chem. Soc. Dalton Trans. 2008, 3, 55705576.

[14] Y. Zhang, C. Chen, S. C. Ghosh, Y. Li, S. H. Hong, Organometallics 2010, 29, 1374-1378.

[15] S. Sabater, Multifunctional N-Heterocyclic Carbene Metal Complexes for the Design of Improved Catalytic Processes, PhD. Thesis, Jaume I University, Spain, 2015.

[16] M. J. Frish, Gaussian 09, Revision D.02, Gaussian Inc., Walligford CT, 2009.

[17] Y. Zhao, D. G. Truhlar, Theor. Chem. Acc. 2008, 120, 215-241. 
[18] D. Andrae, U. Häussermann, M. Dolg, H. Stoll, H. Preuss, Theor. Chim. Acta 1990, 77, 123-141.

[19] A. W. Ehlers, M. Böhme, S. Dapprich, A. Gobbi, A. Höllwarth, V. Jonas, K. F. Köhler, R. Stegmann, A. Veldkamp, G. Frenking, Chem. Phys. Lett. 1993, 208, 111-114.

[20] P. C. Hariharan, J. A. Pople, Theor. Chim. Acta 1973, 28, 213-222.

[21] M. M. Francl, W. J. Pietro, W. J. Hehre, J. S. Binkley, M. S. Gordon, D. J. DeFrees, J. A. Pople, J. Chem. Phys. 1982, 77, 3654-3665.

[22] A. V. Marenich, C. J. Cramer, D. G. Truhlar, J. Phys. Chem. B 2009, 113, 63786396.

[23] R. C. Clark, J. S. Reid, Acta Crystallogr. Sect. A 1995, 51, 887-897.

[24] O. V. Dolomanov, L. J. Bourhis, R. J. Gildea, J. A. K. Howard, H. Puschmann, J. Appl. Crystallogr. 2009, 42, 339-341.

[25] G. M. Sheldrick, Acta Crystallogr. Sect. A Found. Crystallogr. 2008, 64, 112122. 


\section{CHAPTER 7}

Resumen

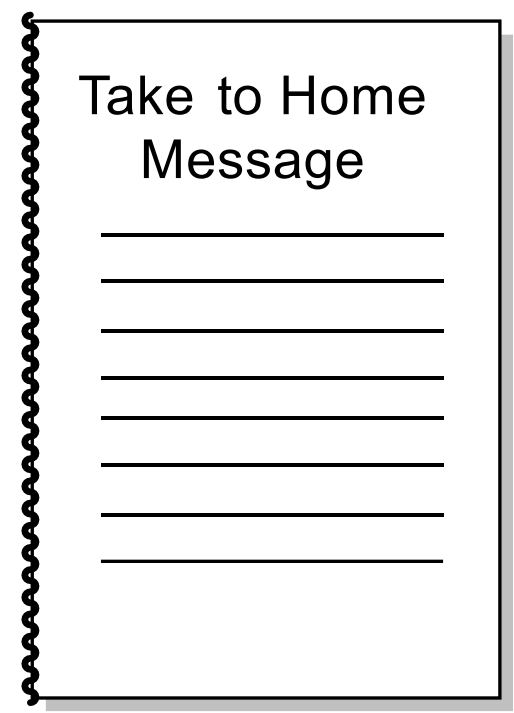



En aplicación de la normativa de estudios de doctorado sobre la elaboración de Tesis Doctorales según el programa RD 99/2011, por la que se establece que: la Tesis Doctoral escrita en una lengua diferente del valenciano o del castellano, en el momento de ser depositada, debe contener un apartado suficientemente amplio en una de estas dos lenguas, y debe formar parte de la encuadernación de la tesis; el siguiente capítulo contiene un resumen en castellano del trabajo recogido en la presente Tesis Doctoral.

\subsection{INTRODUCCIÓN}

Es innegable que la catálisis tiene un papel fundamental en nuestra vida diaria. Actualmente casi cualquier sustancia química en la que podamos pensar tiene al menos un paso catalítico en su producción. ${ }^{[1]}$

La catálisis se divide habitualmente en homogénea - si los sustratos y el catalizador se encuentran en la misma fase - o heterogénea - si sustratos y catalizador están en diferentes fases-. La catálisis homogénea se suele asociar con catalizadores más activos y selectivos. Los catalizadores homogéneos constan frecuentemente de un metal rodeado de ligandos. El diseño de ligandos adecuados permite modular las propiedades del catalizador dando lugar a sistemas muy activos. Además, los catalizadores homogéneos son moléculas bien definidas, lo que permite estudiar en detalle los mecanismos de reacción para, en el siguiente paso, diseñar mejores catalizadores.

A pesar de las ventajas de la catálisis homogénea, actualmente el $90 \%$ de los procesos industriales catalíticos hacen uso de catalizadores heterogéneos. ${ }^{[2]}$ En catálisis heterogénea los reactivos y los catalizadores están en fases diferentes, de manera que el catalizador puede ser separado de manera sencilla al final de la reacción. Habitualmente, los catalizadores heterogéneos son óxidos metálicos o nanopartículas metálicas soportadas en una matriz sólida. El uso de sistemas bifásicos puede dificultar el acceso de los reactivos a los centros catalíticos, lo que afecta a la velocidad de reacción. Para paliar este efecto normalmente se recurre a condiciones de reacción más severas (aumento en la temperatura y/o la presión), lo que implica procesos energéticamente más costosos. No obstante, a pesar de estos inconvenientes los catalizadores heterogéneos están ampliamente aceptados por la industria debido a su alta estabilidad y fácil recuperación.

Sin embargo, con el objetivo de desarrollar procesos más sostenibles, una parte importante del mundo académico ha tratado de buscar estrategias que 
eficientemente combinen la alta actividad y selectividad de los catalizadores homogéneos con la fácil separación y estabilidad de los catalizadores heterogéneos.

De entre las diferentes aproximaciones propuestas para el reciclado de catalizadores homogéneos, la que más ampliamente se ha estudiado es el anclaje de complejos moleculares sobre materiales sólidos. Esta inmovilización se puede llevar a cabo mediante interacciones covalentes o no covalentes. El uso de las interacciones covalentes presenta la ventaja de que la fuerza que mantiene al catalizador anclado al soporte es un fuerte enlace covalente con uno de los ligandos, lo que garantiza que el complejo no se va a separar del material. ${ }^{[3,4]}$ Sin embargo, para la formación de este tipo de anclaje es necesario modificar químicamente el material y/o los ligandos. El uso de esta aproximación conlleva rutas sintéticas más largas para la obtención de los catalizadores, y además implica la modificación también del entorno de coordinación del catalizador, lo que puede afectar a su actividad. Como alternativa, se pueden emplear interacciones no covalentes para anclar especies homogéneas sobre materiales sólidos. Estas interacciones no covalentes suelen ser más débiles, por lo que es necesario un diseño adecuado del catalizador para garantizar que éste no se va a separar del material. La principal ventaja de esta estrategia reside en la fácil preparación de los materiales, los cuales se obtienen muchas veces mediante simple agitación en un disolvente del complejo molecular con el material utilizado como soporte. ${ }^{[5]}$

Los materiales más empleados como soporte son las sílices mesoporosas o los poliestirenos modificados. ${ }^{[6]}$ Alternativamente, en los últimos años existe un creciente interés por el uso de grafeno como soporte. ${ }^{[7,8]}$ El grafeno es un material compuesto por una lámina de átomos de carbono en empaquetamiento hexagonal con una extensa nube de electrones $\pi .^{[9]} \mathrm{El}$ descubrimiento del grafeno supuso una revolución en casi todas las áreas del mundo científico debido a sus excelentes propiedades eléctricas y mecánicas. Sin embargo, la fabricación de grafeno perfectamente puro es extremadamente cara, lo que dificulta su uso en muchas áreas. Como alternativa, existen los grafenos químicamente modificados. Estos materiales consisten en láminas de grafeno que contienen numerosas funcionalidades oxigenadas. Los más ampliamente utilizados son el óxido de grafeno (GO) y el óxido de grafeno reducido (rGO) (Figura 7.1). 


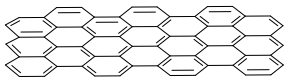

Grafeno

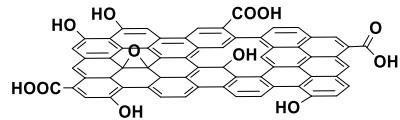

Óxido de grafeno (GO)

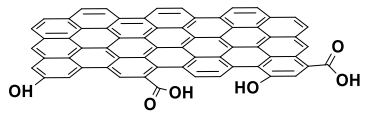

Óxido de grafeno reducido $(\mathrm{rGO})$

Figura 7.1 Representación esquemática de la estructura del grafeno y materiales derivados

La principal diferencia entre el GO y el rGO es el número de grupos funcionales oxigenados presentes en la superficie. La mayor presencia de estas funcionalidades en el GO hace de éste un material adecuado para la inmovilización de catalizadores mediante interacciones covalentes. ${ }^{[10-18]}$ Por el contrario, el rGO, con menos grupos funcionales y una mayor superficie específica, es un buen candidato para inmovilizar catalizadores mediante interacciones no-covalentes. Estas interacciones no-covalentes incluyen interacciones electroestáticas, fuerzas de adsorción, interacciones supramoleculares, e interacciones de apilamiento $\pi$. Debido a la extensa nube de electrones $\pi$ en el rGO y a la elevada área superficial de este material, las interacciones de apilamiento $\pi$ pueden ser empleadas para mantener a los catalizadores unidos al rGO. ${ }^{[19]}$

Las interacciones de apilamiento $\pi$ ocurren cuando un grupo poliaromático entra en contacto con la red de carbonos en hibridación $\mathrm{sp}^{2}$ presente en la superficie del rGO. ${ }^{[20]}$ Por tanto, para que la inmovilización se pueda llevar a cabo es necesario preparar un catalizador con un grupo poliaromático, en general pireno, el cual se puede introducir mediante dos o tres pasos de reacción utilizando procesos bien establecidos y partiendo de reactivos comerciales. ${ }^{[7]}$

La inmovilización de catalizadores homogéneos mediante interacciones de apilamiento $\pi$ se ha estudiado ampliamente utilizando nanotubos de carbono como soporte. ${ }^{[21-26]}$ Sin embargo, el empleo de rGO como soporte permanecía prácticamente inexplorado al inicio de la presente tesis doctoral. De hecho, el único trabajo que describe la inmovilización de catalizadores homogéneos sobre rGO mediante interacciones de apilamiento $\pi$ fue descrito por nuestro grupo en 2014 . $^{\text {[27] }}$ En este trabajo se lleva a cabo la inmovilización de complejos de rutenio y paladio sobre rGO (Figura 7.2). Ambos complejos tienen coordinado al centro metálico un ligando de tipo carbeno $\mathrm{N}$-heterocíclico (NHC) que presenta un grupo pireno. La presencia de este pireno permite el anclaje de los complejos moleculares sobre el rGO bajo condiciones suaves de reacción. 

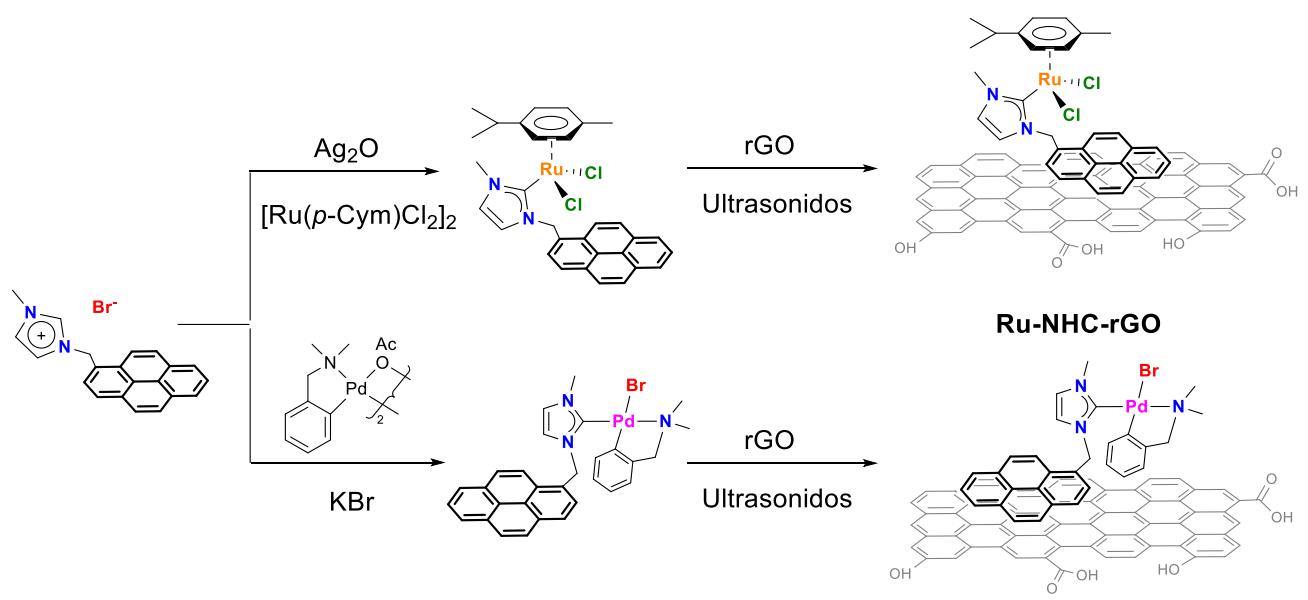

Pd-NHC-rGO

Figura 7.2 Síntesis de los nuevos materiales híbridos Ru-NHC-rGO y Pd-NHC-rGO

Las propiedades catalíticas de los nuevos materiales híbridos fueron estudiadas en las reacciones de oxidación de alcoholes (rutenio) y de reducción de alquenos y grupos nitro (paladio). En ambos casos se pudo reciclar eficientemente el catalizador 10 ciclos catalíticos sin aparente pérdida de actividad. 


\subsection{OBJETIVOS}

En los últimos años el grupo de investigación se ha centrado en el estudio de nuevas metodologías para el reciclado de catalizadores homogéneos. Teniendo en cuenta los precedentes del grupo, el objetivo general de esta tesis es: desarrollar nuevos materiales híbridos basados en catalizadores homogéneos anclados sobre rGO mediante interacciones no covalentes y estudiar sus propiedades catalíticas. Este objetivo general puede dividirse en:

- Diseño y síntesis de nuevas sales de imidazolio con grupos poliaromáticos que servirán como precursores de ligando para la síntesis de complejos metálicos.

- Coordinación de ligandos NHC a diferentes fragmentos metálicos.

- Inmovilización de los nuevos complejos sobre óxido de grafeno reducido.

- Estudio de las propiedades catalíticas de los nuevos materiales híbridos, prestando especial atención al estudio de la reciclabilidad.

- Caracterización de los materiales después de la catálisis para determinar el mecanismo de desactivación de los catalizadores. 


\subsection{RESULTADOS Y DISCUSIÓN}

\subsubsection{Deshidrogenación catalítica de alcoholes}

La Figura 7.3 muestra la síntesis de los complejos de rutenio $1 \mathbf{A}, \mathbf{2 B}$ y $\mathbf{3 C}$ y su inmovilización sobre $\mathrm{rGO}$. Los tres compuestos de Ru difieren en el grupo aromático del ligando NHC. Partiendo de las correspondientes sales de imidazolio, los complejos $1 \mathrm{~A}, \mathbf{2 B}$ y $\mathbf{3 C}$ se sintetizaron por transmetalación con óxido de plata. ${ }^{[28]} \mathrm{A}$ continuación, los complejos se inmovilizaron sobre rGO mediante interacciones de apilamiento $\pi \cdot{ }^{[27,29]}$ Para ello, el rGO se suspendió en diclorometano y se sonicó durante 30 minutos. Seguidamente, se añadió el complejo de rutenio (cálculos realizados para una cantidad máxima soportada del $10 \%$ en peso), se sonicó durante 10 minutos más y posteriormente se agitó a temperatura ambiente toda la noche. De este modo se obtuvieron los materiales $\mathbf{1 A - r G O , ~ 2 B - r G O ~ y ~} \mathbf{3 C}-\mathrm{rGO}$ como sólidos negros.

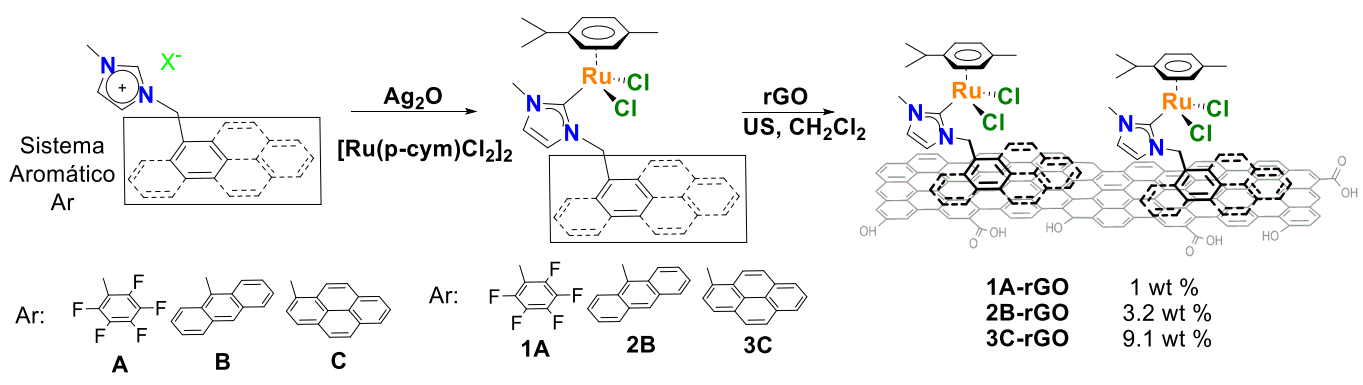

Figura 7.3 Síntesis de complejos de Ru e immobilización sobre rGO

Los materiales se caracterizaron por microscopía electrónica de transmisión de alta resolución (HRTEM) y espectroscopía ultravioleta/visible (UV-Vis), y la cantidad exacta de complejo de rutenio depositada sobre el rGO se determinó por espectrometría de masas con plasma acoplado inductivamente (ICP-MS). En el caso del complejo $1 \mathbf{A}$ con un grupo pentafluorobencilo la interacción de apilamiento $\pi$ es débil y sólo un $1 \%$ en peso del complejo se soporta. En el caso del complejo $2 \mathbf{B}$ (con un grupo antraceno) la cantidad soportada aumenta hasta el $3.2 \%$ en peso. El complejo que se soporta de manera más eficiente es el 3 C $(9.1 \%)$ debido a la fuerte interacción de apilamiento $\pi$ entre el grupo pireno y la extensa nube de electrones $\pi$ del rGO. Con la realización de este estudio se ha comprobado que bajo las mismas condiciones, el grado de deposición depende del número de anillos aromáticos presentes en el ligando NHC (Figura 7.3). Cuanto mayor es la interacción $\pi$ entre el 
grafeno y el anillo poliaromático mayor es la cantidad de compuesto molecular retenido.

La actividad catalítica de los complejos de rutenio y los materiales híbridos se evaluó en la reacción de deshidrogenación de alcoholes primarios en medio acuoso para generar carboxilatos. Esta reacción fue descrita por el Prof. D. Milstein por primera vez en 2013 (Figura 7.4). ${ }^{[30]}$ Tradicionalmente, los alcoholes se oxidan empleando sales metálicas como el dicromato de potasio o el permanganato de potasio, lo que da lugar a elevadas cantidades de residuos que hay que tratar. ${ }^{[31]}$ La ventaja fundamental del sistema descrito por Milstein es que evita la generación de residuos, ya que el único subproducto liberado es hidrógeno, el cual tiene un alto valor añadido para la industria e incluso tiene potenciales aplicaciones como combustible. $^{[32]}$

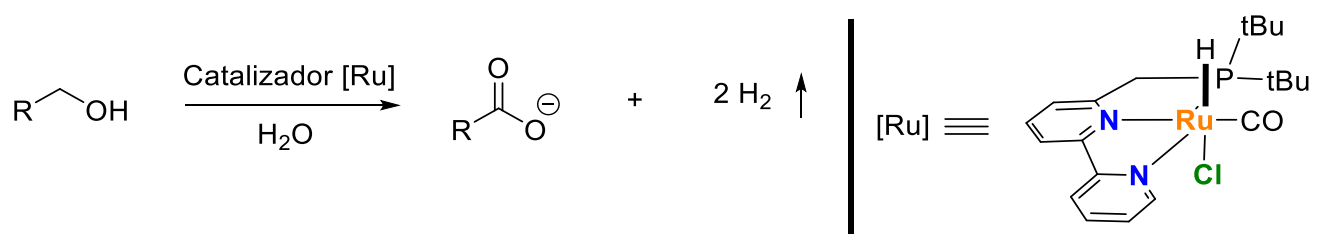

Figura 7.4 Deshidrogenación catalítica de alcoholes descrita por Milstein y colaboradores

En primer lugar, se estudió la actividad de los tres complejos moleculares de rutenio en la reacción de deshidrogenación de alcohol bencílico. Utilizando una carga de catalizador de entre 0.1 y 2 mol \%, los tres complejos presentan moderada actividad, generando el correspondiente carboxilato de manera selectiva. Sin embargo, cuando se utilizan los materiales híbridos 1A-rGO, 2B-rGO y 3C-rGO bajo las mismas condiciones de reacción se observa un incremento en la actividad respecto a los complejos moleculares. Este resultado indica que el hecho de anclar los catalizadores homogéneos de $\mathrm{Ru}$ sobre $\mathrm{rGO}$ da lugar a una mejora en las propiedades catalíticas del sistema.

Los tres materiales híbridos se emplearon en la deshidrogenación de diferentes alcoholes bencílicos, obteniéndose los correspondientes ácidos carboxílicos en excelentes rendimientos tras tratamiento ácido.

A continuación, se estudió la posibilidad de reutilizar los tres materiales híbridos en ciclos consecutivos. Los resultados de los estudios realizados en la recuperación y reciclado de los catalizadores varían en función del material híbrido empleado (Figura 7.5). El material 1A-rGO pudo reciclarse 5 veces sin pérdida de actividad, 
pero posteriormente en los ciclos 6 y 7 la actividad disminuye. El catalizador 2B-rGO pudo reciclarse hasta 8 veces sin aparente desactivación, pero en los ciclos 9 y 10 se observa un descenso en la actividad. El mejor material en términos de reciclabilidad es el 3C-rGO el cual pudo reciclarse hasta 10 veces sin desactivarse. Después de la catálisis, los tres catalizadores se analizaron por TEM y por ICP-MS. Para los tres casos el análisis de TEM muestra la ausencia de nanopartículas después de la catálisis, pero el espectro obtenido mediante espectrometría de dispersión de energía de rayos $X$ (EDS) confirma la presencia de rutenio en la superficie del rGO. Estos resultados indican que las especies activas son de naturaleza molecular. Por otro lado el análisis por ICP muestra que para los catalizadores $\mathbf{1 A - r G O}$ y $\mathbf{2 B}-\mathbf{r G O}$ el contenido de rutenio en la superficie del material disminuye tras el experimento de reciclado, lo que explica su desactivación. Por el contrario, el catalizador 3C-rGO muestra un lixiviado de rutenio inferior al $10 \%$ después de 10 ciclos catalíticos, por lo que puede ser reutilizado sin una desactivación apreciable.
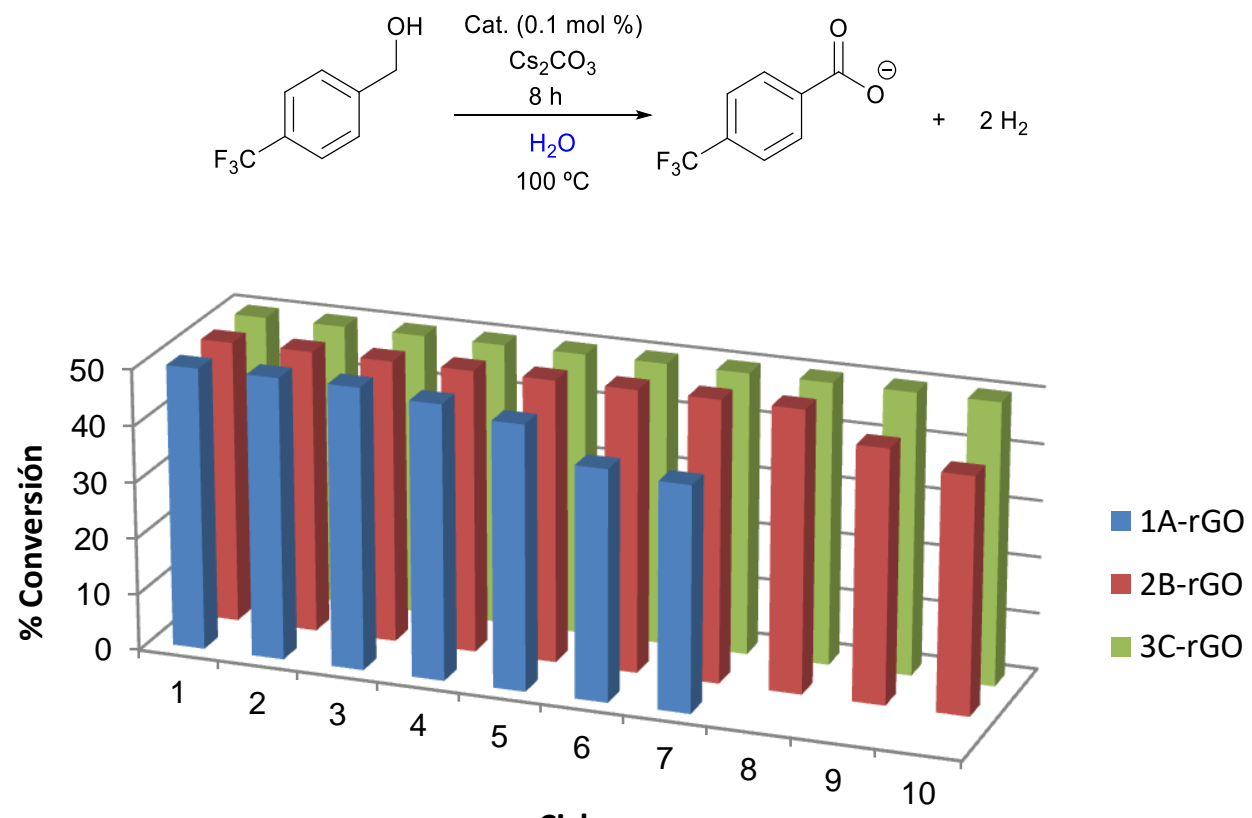

Ciclo

Figura 7.5 Experimentos de reciclado utilizando los materiales 1A-rGO-3C.rGO 


\subsubsection{Deshidrogenación catalítica de aminas para la síntesis de nitrilos}

Dados los buenos resultados obtenidos en la deshidrogenación de alcoholes, se decidió estudiar la deshidrogenación catalítica de aminas para obtener nitrilos. Los nitrilos son importantes grupos funcionales presentes en sustancias farmacéuticas y también son importantes intermedios en síntesis orgánica. ${ }^{[3,34]}$ La Figura 7.6 contiene los catalizadores que se emplearon para el estudio de esta reacción.

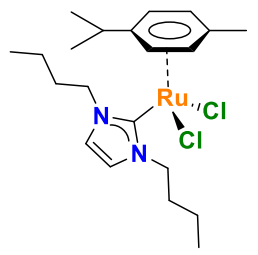

4D

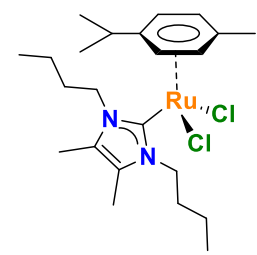

$5 \mathrm{E}$

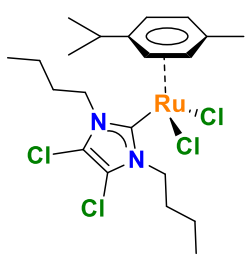

$6 \mathrm{~F}$

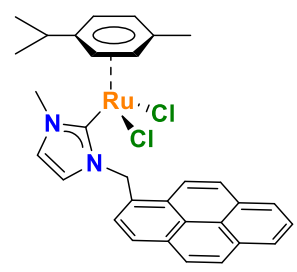

$3 \mathrm{C}$

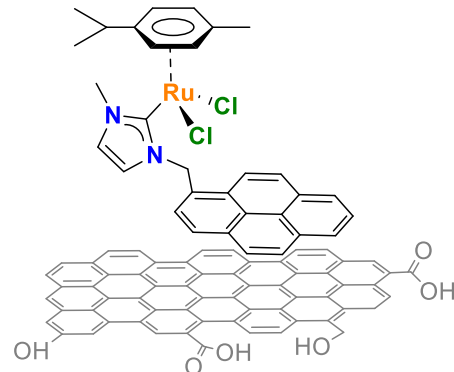

3C-rGo

Figura 7.6 Catalizadores empleados en la deshidrogenación de aminas

Los catalizadores 4D, $5 \mathrm{E}$ y $6 \mathrm{~F}$ tiene la fórmula general $\left[\mathrm{Ru}(p-\mathrm{cym})\left(\mathrm{NHC} \mathrm{Cl}_{2}\right]\right.$, y difieren en el ligando NHC, el cual presenta en todos los casos grupos $n$-butilo como sustituyente en los nitrógenos, pero tiene grupos con diferentes propiedades electrónicas $(\mathrm{H}, \mathrm{Me} \circ \mathrm{Cl}$ ) en las posiciones 4 y 5 del anillo de imidazolideno. Adicionalmente, se introdujo el catalizador $\mathbf{3 C}$ en el estudio para, en el siguiente paso, poder emplear el material híbrido 3C-rGO y tener así un catalizador que pudiese ser reciclado con facilidad.

Inicialmente, se realizaron los ensayos catalíticos para la deshidrogenación de benzilamina utilizando los catalizadores moleculares. Tras la optimización de las condiciones de reacción, se observó que se obtenían elevadas conversiones empleando una carga de catalizador del $2 \mathrm{~mol} \%$ y calentando en tolueno a reflujo durante 8 horas sin necesidad de añadir ningún aditivo (Figura 7.7). La 
deshidrogenación de benzilamina da lugar a dos productos, el correspondiente nitrilo y la imina que resultaría del auto-acoplamiento de dos moléculas de benzilamina Controlar la selectividad de la reacción hacia la formación del nitrilo es uno de los retos a superar para desarrollar una metodología eficaz para la síntesis de nitrilos a partir de aminas. ${ }^{[35-37]}$

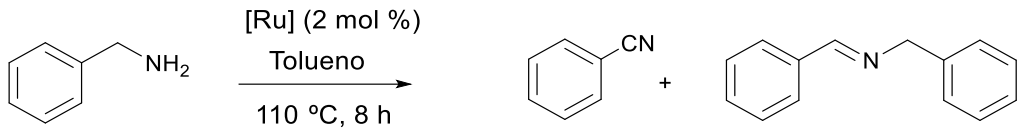

Figura 7.7 Deshidrogenación catalítica de benzilamina

Utilizando los catalizadores 3C, 4D, 5E y 6 F se obtuvieron excelentes conversiones en todos los casos, con selectividades muy parecidas independientemente del catalizador empleado. Los mejores resultados se obtuvieron con el catalizador $\mathbf{3 C}$ el cual a una conversión del $99 \%$ da una relación nitrilo:imina de 82:18 favorable al nitrilo. A continuación, se estudió el efecto que tiene usar benzilaminas sustituidas. Independientemente de si las aminas presentaban un grupo dador o aceptor se obtuvo en todos los casos excelentes conversiones. En cuanto a la quimioselectividad, ésta sigue siendo de moderada a baja. Sin embargo, cuando se emplean aminas alquílicas de cadena larga como sustrato se obtienen mejores resultados en términos de selectividad. Por ejemplo, utilizando hexilamina se consigue una selectividad del $78 \%$ favorable al nitrilo. Si la cadena alifática se incrementa hasta 8 carbonos (octilamina) la selectividad aumenta hasta el $89 \%$, y si se emplean aminas con cadenas más largas se consiguen selectividades del $100 \%$. Estos resultados muestran que excelentes selectividades se obtienen al usar aminas alquílicas de cadena larga y concuerdan con los resultados obtenidos por los profesores. H. García y S. Bellemin-Laponnaz. ${ }^{[38,39]}$

Teniendo en cuenta los buenos resultados obtenidos con el catalizador $\mathbf{3 C}$, se estudió la actividad del catalizador 3C-rGO en la deshidrogenación de bencilamina. Utilizando las mismas condiciones de reacción, se obtuvo conversión completa en 6 horas, lo que supone una reducción del tiempo de reacción en dos horas. Este resultado muestra el efecto positivo que tiene inmovilizar complejos moleculares sobre grafeno. El papel del rGO es probablemente el de estabilizar las especies catalíticamente activas, evitando así ciertos modos de degradación del catalizador. Sin embargo, se requieren más estudios para poder confirmar esta hipótesis. 
Los estudios de reciclabilidad del catalizador 3C-rGO muestran que el material es reciclable durante 8 ciclos sin aparente desactivación. Después del octavo ciclo, la actividad disminuye pero el catalizador es activo durante dos ciclos más. El análisis por TEM de 3C-rGO después de los 10 ciclos muestra que no se han formado nanopartículas. Además, el análisis por EDS confirma la presencia de rutenio en la superficie del grafeno, lo que indica que la especie activa es de naturaleza molecular.

\subsubsection{Generación de hidrógeno a partir de la reacción de acoplamiento de alcoholes e hidrosilanos}

El hidrógeno es una sustancia que ha suscitado mucho interés por sus potenciales aplicaciones como combustible debido a su elevada densidad energética. ${ }^{[40-42]} \mathrm{El}$ hidrógeno puede emplearse como fuente de energía bien utilizándolo en una pila de combustible o bien por combustión directa, generando en ambos casos agua como único subproducto.

El principal problema que lleva asociado el uso de hidrógeno como combustible es su almacenamiento, transporte y manipulación. ${ }^{[43]}$ Bajo condiciones atmosféricas el hidrógeno es un gas altamente inflamable, lo que dificulta su aplicación, sobre todo en el sector de la automoción, uno de los mayores consumidores de energía a nivel global.

Es por ello que se han propuesto diferentes alternativas para una mejor manipulación y almacenamiento del hidrógeno. Entre ellas, en los últimos años destaca el uso de líquidos orgánicos portadores de hidrógeno (LOHC en sus siglas en inglés). ${ }^{[42,44,45]}$ Los LOHC son sustancias que son capaces de liberar parte del hidrógeno contenido en su estructura mediante una reacción catalítica para después poder ser rehidrogenadas y regenerar la sustancia de partida.

En la actualidad se han propuesto diferentes sistemas que pueden actuar como LOHC, sin embargo todos ellos presentan diferentes limitaciones como el uso de condiciones severas de reacción o la imposibilidad de regenerar la sustancia de partida. ${ }^{[46,47]}$ Es por ello que en esta sección se propone el uso de un nuevo LOHC basado en la reacción de acoplamiento de silanos y alcoholes. ${ }^{[48]}$ Esta reacción ha sido estudiada empleando los catalizadores de rutenio 3 C y 3 C-rGo descritos anteriormente. Adicionalmente, también se estudió la reacción utilizando nuevos complejos de iridio anclados sobre grafeno. 


\subsubsection{Acoplamiento de silanos y alcoholes catalizado por especies de rutenio}

La actividad catalítica del complejo $\mathbf{3 C}$ se estudió en primer lugar utilizando como reacción modelo el acoplamiento entre el dimetilfenil silano y diferentes alcoholes primarios (metanol, etanol, propanol y butanol). A efectos prácticos, la reacción se llevó a cabo empleando un exceso del alcohol, el cual se utiliza como disolvente y reactivo (Figura 7.8).

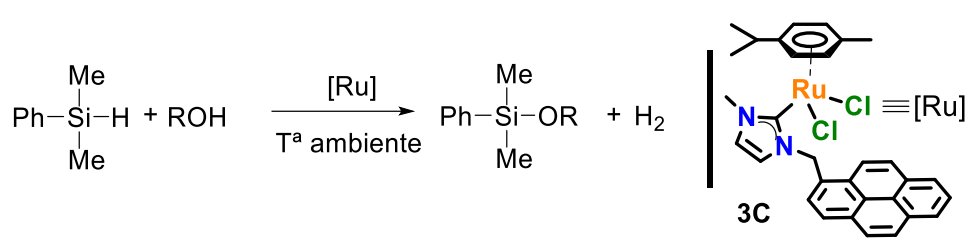

Figura 7.8 Reacción de acoplamiento de hidrosilanos y alcoholes catalizada por complejos de rutenio

Los resultados catalíticos muestran que a temperatura ambiente y utilizando una carga de catalizador del $1 \mathrm{~mol} \%$ la reacción es completa en tiempos cortos de reacción para los 4 alcoholes empleados, liberando hidrógeno de forma cuantitativa y a elevada velocidad. Interesantemente, el silil-eter que se obtiene como producto se puede aislar mediante simple evaporación del disolvente. Además, el hidroxosilano que se podría formar como consecuencia del ataque nucleofílico del agua al hidrosilano no fue observado. ${ }^{[49]}$ Este resultado muestra la elevada selectividad del catalizador $\mathbf{3 C}$, teniendo en cuenta que los alcoholes empleados no fueron destilados y que por tanto tienen un contenido de agua no despreciable.

Dados los buenos resultados, se decidió reducir la cantidad de catalizador a $0.5 \mathrm{~mol} \%$, obteniéndose siempre rendimientos cuantitativos independientemente del alcohol empleado. Sin embargo, cuando la carga de catalizador se redujo al $0.1 \mathrm{~mol} \%$ la reacción seguía siendo completa usando metanol y etanol, pero con $n$-propanol y $n$-butanol se obtienen rendimientos de bajos a moderados. Estos resultados muestran que el hecho de aumentar la longitud de la cadena en el alcohol da lugar a reacciones menos eficientes, probablemente debido a un aumento del impedimento estérico.

A continuación, se decidió estudiar la reacción utilizando otros hidrosilanos. Se estudiaron hidrosilanos primarios (fenilsilano), secundarios (difenilsilano) y terciarios (trifenilsilano), llevando a cabo las reacciones a temperatura ambiente. La tendencia general de estas reacciones es que al aumentar el impedimento estérico en el 
hidrosilano o en el alcohol las reacciones se ralentizan. Por ejemplo, la reacción entre el difenilsilano y el metanol es completa empleando una carga de catalizador de sólo el $0.2 \mathrm{~mol} \%$, pero cuando se emplea etanol es necesario aumentarla hasta el $0.3 \mathrm{~mol} \%$ para obtener conversión completa. Del mismo modo, en el caso del trifenilsilano se obtiene conversión completa empleando metanol y usando una carga del $1 \mathrm{~mol} \%$, pero con los otros alcoholes la conversión varía de moderada a baja incluso cuando se incrementa la temperatura a $45^{\circ} \mathrm{C}$.

Un parámetro importante a tener en cuenta cuando se trata con líquidos portadores de hidrógeno es la cantidad de hidrógeno que se almacena (HSC en sus siglas en inglés para hydrogen storage capacity). El HSC para el par silano/alcohol depende de la estructura de los reactivos empleados. Por ejemplo, para el par $\mathrm{Ph}_{3} \mathrm{SiH} / \mathrm{MeOH}$ el HSC es de solo el 0.7 en peso. En cambio, si se emplean hidrosilanos primarios se incrementa la producción de hidrógeno a 3 moles de $\mathrm{H}_{2}$ por mol de silano. De este modo en el par $\mathrm{PhSiH}_{3} / \mathrm{MeOH}$ el HSC aumenta hasta el $3 \%$ en peso. Estos valores pueden ser aumentados si se utilizan di- o tri- silanos los cuales generan 4 o 6 moles de hidrógeno por mol de silano y mantienen la naturaleza líquida del sistema. ${ }^{[50]}$

A la vista de los buenos resultados obtenidos con $\mathbf{3 C}$, se decidió probar la actividad de $\mathbf{3 C}$-rGo en la reacción de acoplamiento de hidrosilanos y alcoholes con vistas a obtener un catalizador que fuese reciclable. En primer lugar, se comprobó que el rGO por sí mismo no catalizaba la reacción. A continuación, se comparó la actividad del complejo homogéneo y del material híbrido. En la reacción modelo entre el dimetilfenil silano y el metanol, utilizando una baja carga de catalizador del $0.05 \mathrm{~mol} \%$ se obtuvo un rendimiento del $100 \%$ con el catalizador $\mathbf{3 C - r G O}$, mientras que el catalizador $3 \mathrm{C}$ sólo dio un $44 \%$ de rendimiento bajo las mismas condiciones de reacción. Estos resultados muestran el efecto positivo de inmovilizar catalizadores homogéneos sobre rGO mediante interacciones de tipo $\pi$-stacking. Al anclar complejos homogéneos sobre rGO se produce una mejora en las propiedades catalíticas del sistema, debido probablemente a la estabilización de las especies catalíticamente activas. El material 3C-rGO se usó para la reacción de acoplamiento de dimetilfenil silano y fenilsilano con otros alcoholes, dando en todos los casos rendimientos de al menos el $90 \%$ utilizando una carga de catalizador del $0.05 \mathrm{~mol} \%$.

Vista la elevada actividad de 3C-rGO, se analizó la posibilidad de reciclar el catalizador en ciclos consecutivos. La reciclabilidad de $\mathbf{3 C}$-rGO se estudió en la reacción de acoplamiento de dimetilfenil silano y metanol. Después de cada ciclo el 
catalizador se separó por decantación, se lavó con metanol ( $3 \times 5 \mathrm{~mL}$ ) y se empleó en el siguiente ciclo.

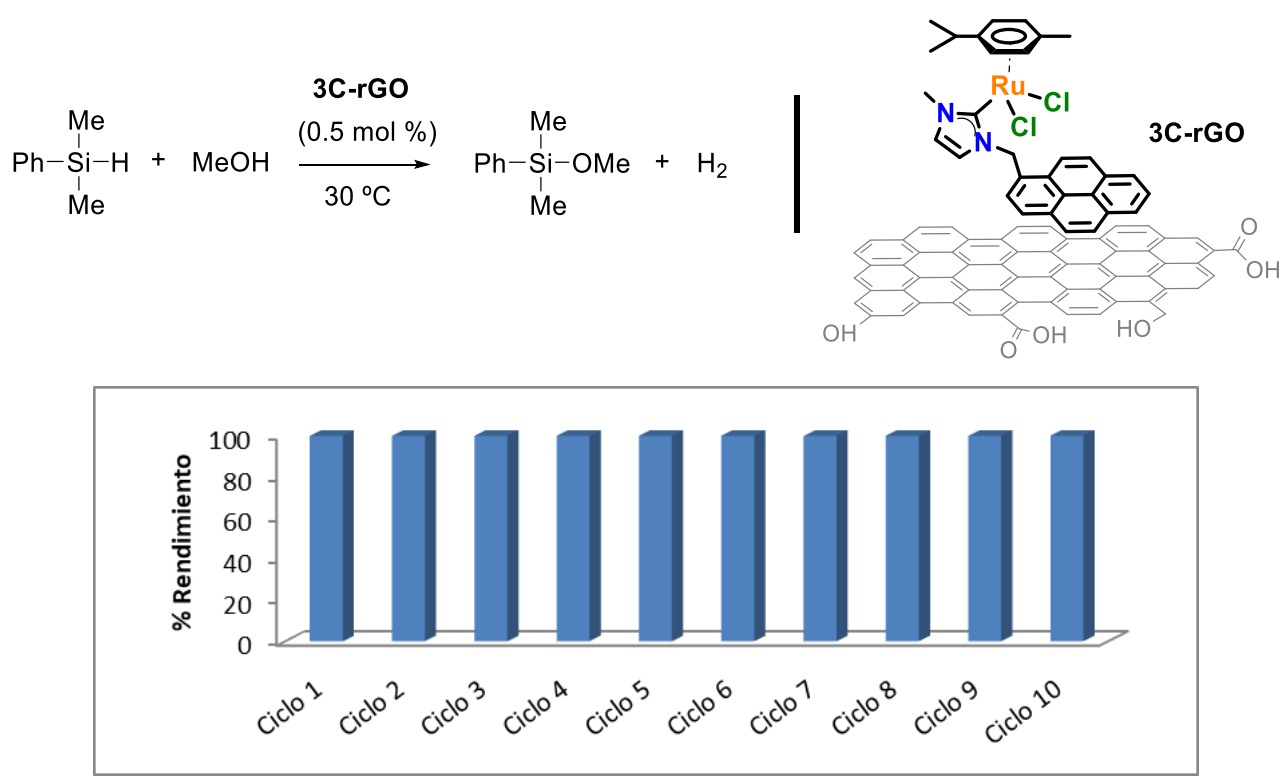

Figura 7.9 Experimento de reciclado utilizando el catalizador 3C-rGO

Utilizando esta estrategia, el catalizador 3C-rGO puede ser reciclado hasta 10 veces sin aparente pérdida de actividad (Figura 7.9). Después del experimento de reciclado, el material fue analizado por HRTEM. Los resultados de HRTEM muestran que el material no ha sufrido cambios morfológicos importantes, y que el complejo de rutenio permanece en la superficie del rGO, ya que el análisis de EDS muestra la presencia de rutenio y no se observan nanopartículas.

\subsubsection{Acoplamiento de silanos y alcoholes catalizado por especies de iridio}

En analogía a la síntesis del complejo 3C, la síntesis del complejo de iridio $\mathbf{8 C}$ se trató de llevar a cabo mediante transmetalación con óxido de plata. Sin embargo, bajo estas condiciones no se obtuvo el complejo deseado sino el producto ortometalado $\mathbf{8 C ^ { \prime }}$ (Figura 7.10). ${ }^{[51]}$ Por ello, se diseñó una estrategia diferente para la síntesis de $\mathbf{8 C}$ que implicaba la deprotonación de la sal de imidazol $\mathbf{C}$ con tert-butóxido de potasio a baja temperatura para después coordinar el carbeno libre generado in situ al centro metálico de iridio. El tratamiendo del complejo $8 \mathrm{C}$ con un equivalente de tert-butóxido de potasio da lugar al producto ortometalado $\mathbf{8} \mathbf{C}^{\prime}$. 


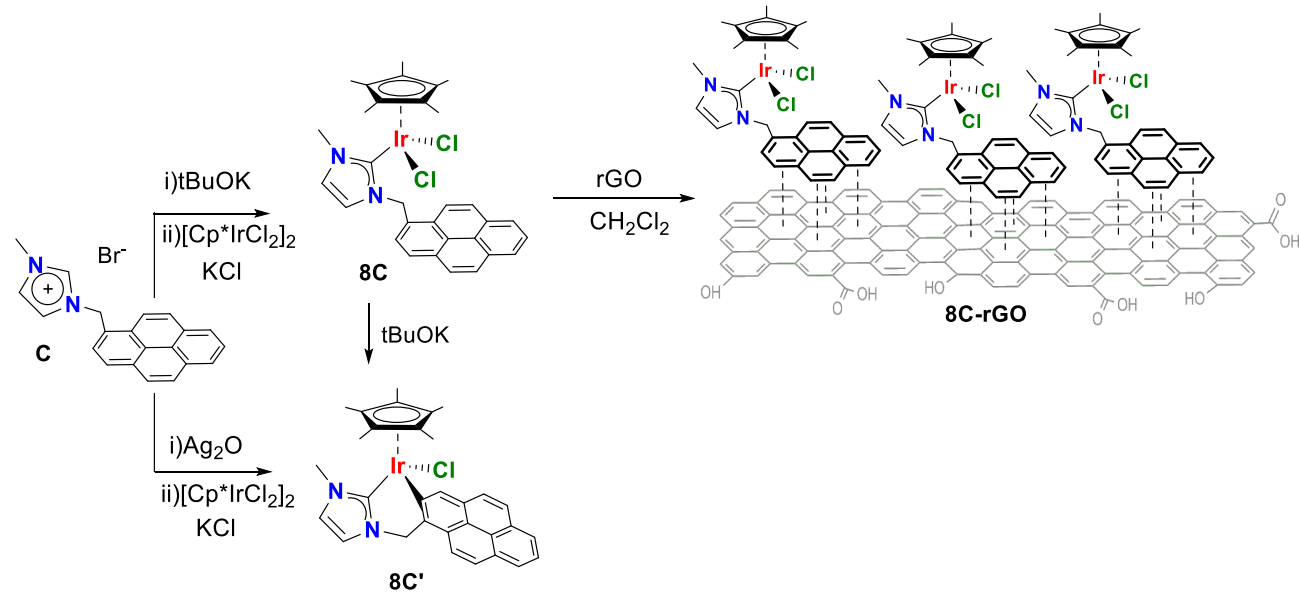

Figura 7.10 Síntesis de los complejos $\mathbf{8 C}$ y $\mathbf{8 C ^ { \prime }}$ y anclaje de $\mathbf{8 C}$ sobre rGO

Los complejos $\mathbf{8 C}$ y $\mathbf{8} \mathbf{C}^{\prime}$ han sido sintetizados por primera vez en nuestro laboratorio y fueron completamente caracterizados por espectroscopía de resonancia magnética nuclear (RMN), espectrometría de masas de alta resolución (HRMS), análisis elemental y difracción de rayos X. Además, el complejo $8 \mathrm{C}$ se ancló sobre rGO siguiendo la misma metodología empleada para $3 \mathrm{C}$, generando el material híbrido 8C-rGO, el cual fue completamente caracterizado mediante HRTEM, espectroscopía fotoelectrónica de rayos X (XPS) e ICP-MS.

Teniendo los complejos $\mathbf{8 C}$ y $\mathbf{8} \mathbf{C}^{\prime}$ y el material $\mathbf{8 C}-\mathbf{r G O}$ sintetizados se inició el estudio de su actividad catalítica en la reacción de acoplamiento de silanos y alcoholes. Para la optimización de las condiciones de reacción se emplearon el dimetilfenil silano y el metanol como sustratos modelo. El complejo $\mathbf{8} \mathbf{C}^{\prime}$ presenta una muy baja actividad por lo que no se utilizó en estudios posteriores. Por el contrario, cuando se emplea 8C como catalizador la reacción inmediatamente empieza a generar hidrógeno. Utilizando una carga de catalizador del $0.5 \mathrm{~mol} \%$ la reacción es completa en 2 minutos, tal y como se comprobó empleando una bureta invertida llena de agua para así poder ver cuando cesa la producción de hidrógeno. Después de la reacción, el silil-eter formado se aisló tras evaporación del disolvente y se analizó por RMN, mostrando la formación de un producto único, el metoxidimetilfenil silano. En el caso de los catalizadores de iridio resulta esencial secar bien los alcoholes sobre tamiz molecular, ya que en presencia de agua se produce la formación del hidroxosilano además del silil éter. 
El complejo $\mathbf{8 C}$ se probó en el acoplamiento de diferentes silanos y alcoholes. La diversidad estructural de los silanos permite obtener mezclas silano/alcohol con diferente HSC (Tabla 7.1).

Tabla 7.1 Acoplamiento de diferentes silanos y alcoholes catalizado por 8C

$$
\mathrm{R}_{4-n} \mathrm{SiH}_{n}+n \mathrm{R}^{\prime} \mathrm{OH} \underset{30^{\circ} \mathrm{C}}{\stackrel{[\mathrm{Ir}]}{\longrightarrow}} \mathrm{R}_{4-n \mathrm{Si}\left(\mathrm{OR}^{\prime}\right)_{n}+\mathrm{n} \mathrm{H}_{2}}
$$

\begin{tabular}{|c|c|c|c|c|c|}
\hline Entrada & Silano & ROH & effHSC (\%) & $\mathrm{t}$ (min) & Rendimiento(\%)a \\
\hline 1 & & $\mathrm{MeOH}$ & 1.2 & 3 & $100(98)$ \\
\hline 2 & & $\mathrm{EtOH}$ & 1.1 & 12 & $100(97)$ \\
\hline 3 & & $n \mathrm{PrOH}$ & 1.0 & 40 & $100(98)$ \\
\hline 4 & & $n \mathrm{BuOH}$ & 0.96 & 55 & 100 (99) \\
\hline 5 & & $\mathrm{MeOH}$ & 1.4 & 20 & 100 \\
\hline 6 & & $\mathrm{EtOH}$ & 1.2 & 30 & 100 \\
\hline 7 & $\mathrm{Ph}_{2} \mathrm{SiH}_{2}$ & $\mathrm{MeOH}$ & 1.6 & 120 & $85(82)$ \\
\hline 8 & & $\mathrm{MeOH}$ & 3.0 & 5 & 100 \\
\hline 9 & & $\mathrm{EtOH}$ & 2.5 & 15 & $100(94)$ \\
\hline 10 & & $\mathrm{MeOH}$ & 2.8 & 20 & $100(93)$ \\
\hline 11 & & EtOH & 2.3 & 35 & $100(90)$ \\
\hline 12 & & $\mathrm{MeOH}$ & 3.3 & 15 & $100(75)$ \\
\hline 13 & & $\mathrm{EtOH}$ & 2.7 & 20 & $96(80)$ \\
\hline 14 & & $\mathrm{MeOH}$ & 4.3 & 12 & $100(96)$ \\
\hline $15^{b}$ & & EtOH & 3.3 & 30 & $100(97)$ \\
\hline $16^{c}$ & & $n \mathrm{PrOH}$ & 2.7 & 25 & 100 \\
\hline $17^{c}$ & & $n \mathrm{BuOH}$ & 2.3 & 40 & 100 \\
\hline
\end{tabular}

Condiciones de reacción: hidrosilano $(1.0 \mathrm{mmol})$, catalizador de iridio $8 \mathrm{C}(0.5 \mathrm{~mol} \%), 30{ }^{\circ} \mathrm{C}$ y $1 \mathrm{~mL}$ de alcohol. [a] Rendimientos determinados en base a la formación de $\mathrm{H}_{2}$. Entre paréntesis se expresan los rendimientos aislados basados en la cantidad de silil éter determinada por RMN de protón utilizando 1,3,5 trimetoxi benceno como patrón. [b] Catalizador de iridio $1 \mathrm{~mol} \%$. [c] Catalizador de iridio $2 \mathrm{~mol} \%$.

El complejo 8C es capaz de llevar a cabo la reacción de acoplamiento de diferentes alcoholes y silanos de manera eficiente (Tabla 7.1). Las reacciones son siempre más rápidas empleado metanol como alcohol. A medida que aumenta la cadena alifática del alcohol la cinética de la reacción se ve afectada, pero se obtienen rendimientos 
altos o cuantitativos en todos los casos. Especialmente interesante es el par 1,4 disilabutano/MeOH. Este disilano es capaz de liberar 6 moles de hidrógeno por cada mol de silano, de manera que el HSC del sistema es $4.3 \%$ en peso. Este contenido de hidrógeno es muy elevado y se acerca al HSC de uno de los líquidos orgánicos portadores de hidrógeno más estudiados, el ácido fórmico. Sin embargo, a diferencia del ácido fórmico, el par 1,4 disilabutano/MeOH presenta la ventaja de que no genera un mol de $\mathrm{CO}_{2}$ (un gas asociado al efecto invernadero) por cada mol de $\mathrm{H}_{2}$ que libera. ${ }^{[52]}$

El siguiente paso en este trabajo fue el de estudiar la actividad y reciclabilidad del catalizador 8C-rGO. Utilizando una carga de catalizador del $0.5 \mathrm{~mol} \%$ en la reacción modelo entre el dimetilfenil silano y el metanol se obtuvo el correspondiente silil-éter de forma cuantitativa en 7 minutos. Habitualmente, cuando se inmoviliza un catalizador molecular sobre un soporte sólido tiene lugar un descenso en la actividad debido a problemas de transferencia de masa hacia los centros catalíticamente activos. Sin embargo, en el caso del rGO se está trabajando con un material 2D que no impide el acceso a los centros activos. De este modo, la actividad catalítica del complejo molecular $8 \mathrm{C}$ (100\% de rendimiento tras 3 minutos) es comparable con la actividad del catalizador soportado 8C-rGo $(100 \%$ de rendimiento en 7 minutos).

Vista la elevada actividad de $\mathbf{8 C}$-rGo se estudió la reciclabilidad en ciclos consecutivos. Los resultados muestran que el catalizador $\mathbf{8 C}$-rGO se puede reciclar 10 veces sin aparente pérdida de actividad. El estudio del material después de la catálisis por HRTEM muestra que el rGO mantiene su morfología y que no se han formado nanopartículas de iridio. Esta observación indica que el catalizador molecular no se degrada bajo las condiciones de reacción y sigue presente en la superficie del rGO después de la catálisis, tal como indica el espectro de EDS.

\subsubsection{Catalizadores de oro en la superficie de grafeno}

En el transcurso de este trabajo de investigación se decidió estudiar la actividad de diferentes catalizadores de oro inmovilizados en la superficie de grafeno. En particular se estudió la actividad de complejos organometálicos y nanopartículas de oro sobre rGO.

\subsubsection{Complejos moleculares de oro anclados sobre rGO}

La Figura 7.11 muestra la síntesis de los dos complejos de oro que se emplearon en este estudio. Los dos complejos presentan estructuras muy similares pero difieren 
en uno de los sustituyentes de los nitrógenos del anillo NHC (metilo o mesitilo). Se siguieron dos metodologías de síntesis diferentes. Mientras que el complejo 9C fue obtenido por trasmetalación con óxido de plata, ${ }^{[28]}$ el complejo $\mathbf{1 0 H}$ se sintetizó mediante deprotonación de la sal de imidazol $\mathbf{H}$ con carbonato de potasio en presencia del precursor metálico $\mathrm{AuCl}\left(\mathrm{SMe}_{2}\right) .{ }^{[53]}$ Ambos compuestos fueron completamente caracterizados por RMN, HRMS, análisis elemental, y difracción de rayos $X$.

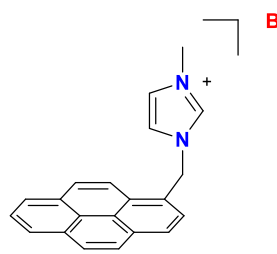

C

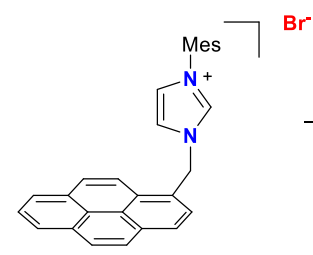

H

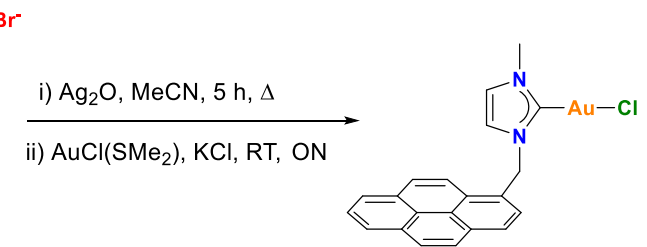

9C

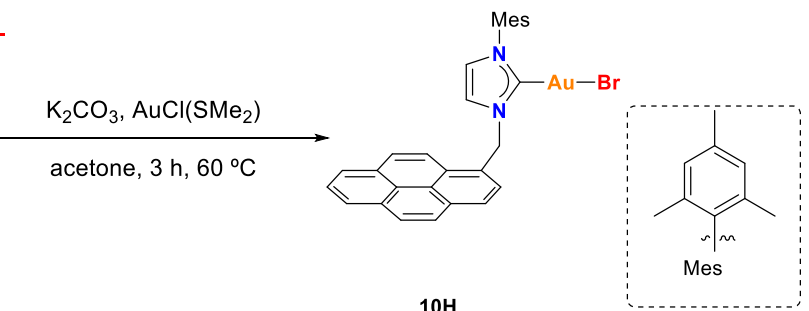

Figura 7.11 Síntesis de los complejos $\mathbf{9 C}$ y $\mathbf{1 0 H}$

Siguiendo la metodología descrita por nuestro grupo, los dos complejos se inmovilizaron sobre $\mathrm{rGO}$. Los nuevos materiales híbridos $\mathbf{9 C}-\mathrm{rGO}$ y $\mathbf{1 0 H}-\mathbf{r G O}$ fueron completamente caracterizados mediante HRTEM, XPS e ICP-MS. EI análisis microscópico revela que la morfología del rGO se preserva después del proceso de inmovilización y que no se forman nanopartículas, lo que indica que el material está compuesto por el complejo molecular de oro inmovilizado sobre la superficie del rGO. Esta hipótesis es confirmada por los análisis de espectroscopía fotoelectrónica de rayos $X$, dónde se observan los picos del oro y nitrógeno a la misma energía de enlace tanto para el complejo molecular como para el material híbrido.

Cuando se tuvo sintetizados y caracterizados los complejos moleculares ( $\mathbf{9 C}$ y $\mathbf{1 0 H}$ ) y los materiales híbridos (9C-rGO y $\mathbf{1 0 H - r G O )}$ se realizaron los ensayos catalíticos. La reacción estudiada fue la hidroaminación intramolecular de 2-alquinil anilinas. Cuando se emplean complejos del tipo NHC-Au-X (X $=\mathrm{Cl}$ o $\mathrm{Br}$ ) es necesario añadir un abstractor de haluros que genere la especie catiónica de $\mathrm{Au}$ (I), la cual es la 
responsable de la catálisis. En este caso el abstractor de haluros empleado fue el tetrafluoroborato de plata $\left(\mathrm{AgBF}_{4}\right){ }^{[54]}$

Para analizar en detalle el efecto del soporte y de modificar el ligando se obtuvieron perfiles de reacción para los 4 catalizadores (Figura 7.12).

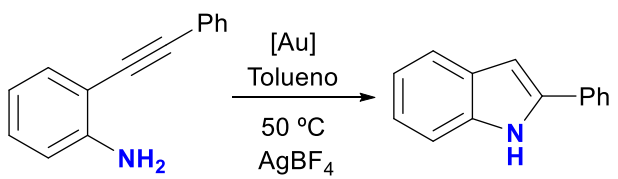

a)
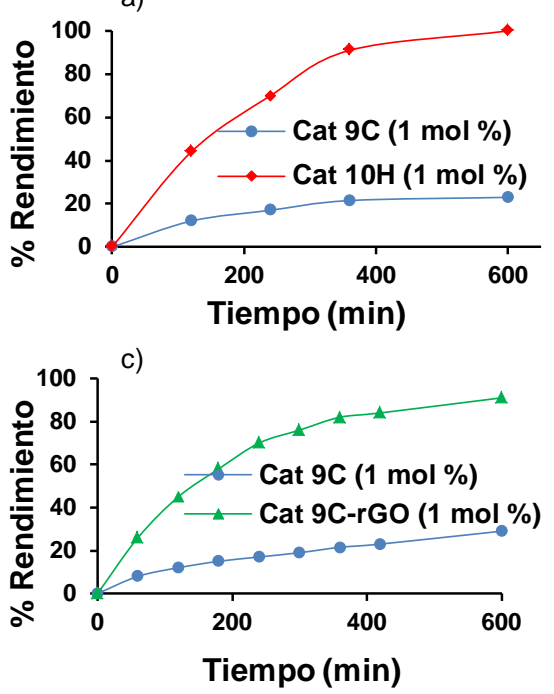

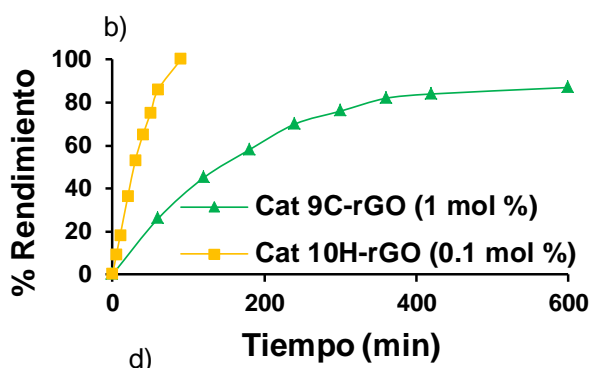

d)

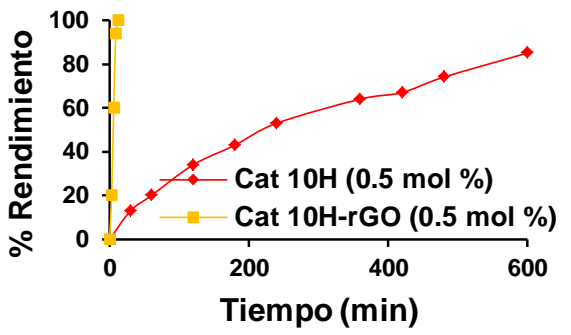

Figura 7.12 Seguimiento de la reacción de hidroaminación intramolecular empleando diferentes catalizadores de oro

En primer lugar se examinó el efecto del ligando, tanto a nivel molecular como cuando los catalizadores están soportados sobre rGO. Los resultados muestran que cambiar un grupo metilo por un grupo mesitilo en el anillo de NHC tiene un efecto importante sobre las propiedades catalíticas del sistema. El complejo 9C con sustituyente metilo es un catalizador poco activo en la reacción de hidroaminación intramolecular (Figura 7.12, a). En cambio, al sustituir un grupo metilo por un mesitilo se produce una importante mejora en las propiedades catalíticas, alcanzándose rendimientos cuantitativos. Esta misma tendencia fue observada cuando los complejos $\mathbf{9 C}$ y $\mathbf{1 0 H}$ se encuentran anclados sobre rGO (Figura 7.12, b). Estos resultados ilustran perfectamente la importancia que tiene un diseño de ligandos adecuado en la catálisis homogénea. 
A continuación, se analizó el efecto que tiene anclar los complejos moleculares sobre rGO en las propiedades catalíticas. Cuando se emplea el complejo molecular 9C a una carga de catalizador de 1 mol \% se obtienen bajos rendimientos ( $\approx 20 \%$ ) tras $10 \mathrm{~h}$ de reacción. En cambio, si se emplea el material híbrido 9C-rGo, se obtienen rendimientos superiores al $80 \%$ bajo las mismas condiciones de reacción (Figura $7.12, \mathrm{c}$ ). Este efecto es incluso más pronunciado en el caso del complejo $\mathbf{1 0 H}$ y el material híbrido $\mathbf{1 0 H}-\mathbf{r G O}$. El complejo $\mathbf{1 0 H}$ es un catalizador activo en la reacción de hidroaminación intramolecular. Utilizando una carga de catalizador del $0.5 \mathrm{~mol} \% \mathbf{1 0 H}$ da rendimientos cercanos al $80 \%$ tras 10 horas de reacción. Sin embargo, estos resultados mejoran drásticamente cuando se utiliza 10H-rGO, el cual da rendimientos cuantitativos en sólo 12 minutos bajo las mismas condiciones de reacción. Estos resultados demuestran el efecto positivo que tiene inmovilizar complejos de oro sobre la superficie de rGO.

Seguidamente, se estudió la reciclabilidad del material híbrido 10H-rGo. Las reacciones se llevaron a cabo bajo las condiciones ilustradas en la Figura 7.12. Cuando finalizó la reacción el material catalítico fue separado por decantación lavado con tolueno y empleado en el siguiente ciclo. Usando esta metodología, el catalizador $\mathbf{1 0 H}-\mathbf{r G O}$ se pudo reciclar 3 veces sin aparente pérdida de actividad. Después del tercer ciclo existe una cierta desactivación, pero alargando los tiempos de reacción se puede reciclar el material hasta un total de 6 veces. Tras el experimento de reciclado se analizó el material por microscopía electrónica de transmisión. Los resultados muestran que la morfología del soporte no ha sufrido grandes cambios y que no se han formado nanopartículas, lo que indica la naturaleza molecular de las especies activas. Por otro lado, el análisis por ICP-MS muestra que la desactivación del catalizador se debe al lixiviado del metal de la superficie del soporte durante los ciclos catalíticos.

\subsubsection{Nanopartículas de oro funcionalizadas con ligandos NHC ancladas sobre la superficie de rGO}

Dado el importante efecto observado al introducir grupos aromáticos como $\mathrm{N}$-sustituyentes en el anillo del NHC, se diseñó un complejo de oro (11I) que contenía dos grupos aromáticos voluminosos como $\mathrm{N}$-sustituyentes. De este modo, el fragmento pireno se introdujo través de una funcionalidad éter en la posición 4 del anillo de imidazol (Figura 7.13). 

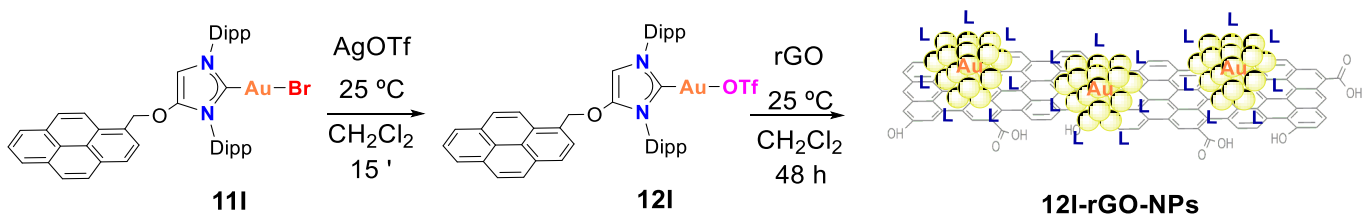

12I-rGO-NPs

Figura 7.13 Síntesis de nanopartículas de oro funcionalizadas con ligandos NHC sobre la superficie de grafeno

Cuando se hubo sintetizado y caracterizado el complejo 11l, el siguiente paso fue el intercambio del bromuro por el ligando trifluorometanosulfonato (OTf). El ligando OTf es más lábil que el bromuro, lo que permite llevar a cabo las reacciones catalíticas sin necesidad de ningún aditivo. ${ }^{[55]}$ El tratamiento del complejo 111 con un equivalente de trifluorometanosulfonato de plata da lugar al complejo 12I (Figura 7.13). Cuando el complejo 12I se trata con una suspensión de grafeno en diclorometano se obtienen resultados completamente diferentes a los obtenidos con el complejo 10H (sección 7.3.4.1). El complejo $12 \mathrm{l}$ es estable en disolución y en estado sólido, pero al ser tratado con rGO descompone y genera nanopartículas de oro que quedan directamente inmovilizadas en rGO. Estas nanopartículas se generan bajo condiciones suaves y en ausencia de agentes reductores, de manera que las propiedades del soporte (rGO) se mantienen, tal y como se observó por microscopía electrónica de transmisión. Las imágenes obtenidas mediante TEM revelan la presencia de pequeñas nanopartículas de oro altamente cristalinas sobre el rGO. Se realizó un estudio estadístico (histograma) que determinó que el tamaño promedio de las NPs es de $3.2 \pm 1.5 \mathrm{~nm}$.

Para comprobar el efecto que tiene el ligando trifluorometanosulfonato en la generación de las NPs se realizó un experimento control en el que se inmovilizaba el complejo 11I (con un ligando bromuro) sobre rGO. Las imágenes de TEM de este material muestran la ausencia de nanopartículas, lo que confirma que el ligando lábil OTf es necesario para la generación de Au-NPs sobre rGO. 
Con fines comparativos, también se sintetizaron nanopartículas de oro sobre rGO en ausencia de ligando NHC. Para ello, se trató el rGO con ácido tetracloroaurico $\left(\mathrm{HAuCl}_{4}\right)$, el cual descompone generando NPs de Au que quedan ancladas sobre el rGO (Figura 7.14). ${ }^{[56]}$
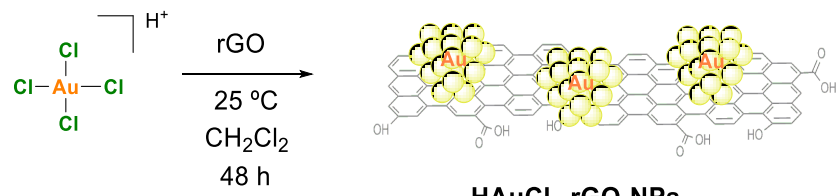

$\mathrm{HAuCl}_{4}$-rGO-NPs

Figura 7.14 Síntesis de nanopartículas de oro sin ligandos immobilizadas sobre rGO

El análisis por microscopía electrónica del material híbrido $\mathrm{HAuCl}_{4}$-rGO-NPs- muestra la formación de nanopartículas de oro de mayor tamaño sobre la superficie del rGO. El análisis estadístico realizado determinó que estas NPs tienen un tamaño promedio de $12.4 \pm 4.2 \mathrm{~nm}$. Puestos en conjunto, estos resultados muestran el efecto importante que tienen los ligandos en la estabilización de nanopartículas de oro. La presencia de ligandos NHC controla el crecimiento de las nanopartículas. En cambio, en ausencia de ligando las nanopartículas crecen sin control, obteniéndose al final unas NPs de un tamaño promedio mayor y con una mayor dispersidad.

La actividad catalítica del complejo 121 y de los materiales 12I-rGO-NPs y $\mathbf{H A u C l}_{4^{-}}$ rGO-NPs se estudió en la reacción de hidratación de alquinos para obtener cetonas. La optimización de las condiciones de reacción se llevó a cabo utilizando 4-octino como sustrato (Figura 7.15).

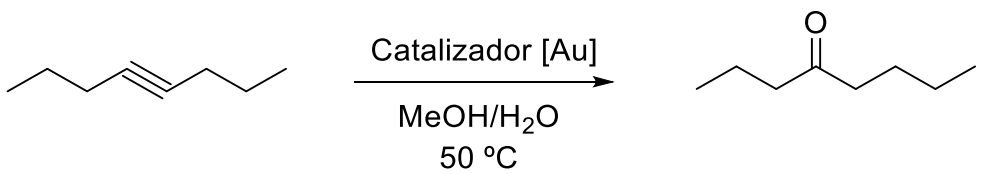

Figura 7.15 Reacción de hidratación de 4-octino catalizada por especies de oro

El complejo $\mathbf{1 2 l}$ es catalíticamente activo en esta reacción, dando lugar a rendimientos cuantitativos en 150 minutos utilizando una carga de catalizador del $0.25 \mathrm{~mol} \%$. Cuando se emplea el material híbrido 12I-rGO-NPs se obtienen mejores resultados catalíticos. En este caso, utilizando una carga de catalizador del 0.05 mol \% la conversión de 4-octino es completa en sólo 60 minutos. Por su parte, el material $\mathbf{H A u C l}_{4}$-rGO-NPs es completamente inactivo en esta reacción, probablemente debido al mayor tamaño de las nanopartículas de oro. El tamaño de las nanopartículas de oro tiene un efecto drástico sobre su actividad catalítica. ${ }^{[57]}$ 
En el caso de 12I-rGO-NPs la presencia de ligandos permite obtener nanopartículas pequeñas que son altamente activas en la hidratación de alquinos. Por el contrario en el material $\mathbf{H A u C l}_{\mathbf{4}}$-rGO-NPs las nanopartículas crecen sin control hasta un tamaño que hace que sean completamente inactivas. Este resultado demuestra el efecto importante que tiene el uso de ligandos y soportes en el diseño de catalizadores de oro basados en nanopartículas.

El catalizador 12I-rGO-NPs se empleó en la hidratación de diferentes alquinos terminales e internos, dando siempre excelentes rendimientos a bajas cargas de catalizador (0.025-0.05 mol \%).

La estabilidad de 12I-rGO-NPs se estudió mediante experimentos de reciclabilidad. Después de cada ciclo el material se separó por decantación, se lavó con metanol y se usó en el siguiente ciclo. De este modo, se pudo reciclar el material tres veces sin aparente pérdida de actividad. Después del tercer ciclo, la actividad decae paulatinamente, pero se obtienen rendimientos cuantitativos durante 7 ciclos más incrementando el tiempo de reacción.

El análisis del material por microscopía electrónica de transmisión después de 10 ciclos catalíticos muestra que el rGO no ha sufrido modificaciones y que las nanopartículas de oro conservan su cristalinidad y su tamaño. El análisis por ICP-MS revela que el lixiviado del metal de la superficie del catalizador es el motivo que explica su desactivación, ya que el $50 \%$ del oro inicial se pierde durante los experimentos de reciclado. 


\subsection{CONCLUSIONES}

En la presente tesis doctoral se ha descrito la síntesis y caracterización de una serie de materiales híbridos basados en complejos moleculares con ligandos NHC inmovilizados sobre rGO mediante interacciones de apilamiento $\pi$.

En primer lugar, se sintetizaron tres complejos de rutenio que presentaban diferentes grupos aromáticos en su estructura (pentafluorofenilo, antraceno o pireno). La eficiencia de la inmovilización de los complejos sobre el rGO depende de la fuerza de la interacción $\pi$ entre el rGO y los grupos aromáticos presentes en el catalizador, la cual aumenta al aumentar el número de anillos aromáticos. La actividad catalítica de los tres materiales híbridos que resultan de la inmovilización de los tres complejos de rutenio se estudió en la reacción de oxidación de alcoholes primarios en medio acuoso. Los resultados muestran que:

- $\quad$ Los materiales híbridos son más activos que los complejos moleculares.

- $\quad$ Los tres materiales híbridos se pueden reciclar.

- La eficiencia de la reciclabilidad depende de la fuerza de la interacción $\pi$ entre el rGO y el complejo molecular, siendo el catalizador que presenta un grupo pireno el más eficiente.

En segundo lugar, se estudió la actividad de diferentes complejos de Ru con ligandos $\mathrm{NHC}$ en la deshidrogenación de aminas. Los complejos del tipo $\left[\mathrm{Ru}(p-\mathrm{cym})(\mathrm{NHC}) \mathrm{Cl}_{2}\right]$ son capaces de deshidrogenar aminas en ausencia de aditivos. Para las aminas bencílicas, se obtienen selectividades moderadas hacia la formación del nitrilo. En cambio, cuando se emplean aminas alquílicas de cadena larga se obtienen mejores selectividades. La reacción de deshidrogenación de aminas también se estudió empleando el material híbrido basado en el complejo molecular de rutenio con un fragmento pireno anclado sobre rGO (3C-rGO). Los resultados muestran que este material híbrido es un catalizador eficaz para la deshidrogenación de aminas, pudiéndose reciclar hasta 8 veces sin pérdida de actividad.

En tercer lugar, se sintetizó un material híbrido basado en un complejo de iridio immobilizado sobre rGO. Tanto el material híbrido de iridio (8C-rGO) como el de rutenio (3C-rGO) fueron estudiados en la reacción de acoplamiento de silanos y alcoholes para producir silil-éteres e hidrógeno. Ambos materiales híbridos son catalizadores muy eficientes para esta reacción, liberando hidrógeno a elevada velocidad. Diferentes combinaciones de silanos y alcoholes reaccionan en presencia de estos catalizadores dando lugar a rendimientos elevados. Especialmente 
interesante es la combinación de 1,4-disilabutano y metanol. Esta mezcla tiene un contenido de hidrógeno cercano al $4.3 \%$ en peso, lo que la hace muy interesante para aplicaciones potenciales como líquido orgánico portador de hidrógeno.

Por último, se ha estudiado la actividad catalítica de complejos y nanopartículas de oro sobre rGO. Los resultados catalíticos muestran que los complejos de oro son mucho más activos cuando se encuentran inmovilizados sobre la superficie del grafeno, dando lugar a rendimientos cuantitativos en menores tiempos de reacción. Además, los nuevos materiales híbridos basados en oro se pueden reciclar en ciclos sucesivos sin inicial pérdida de actividad. Estudios adicionales muestran que el contraion presente en el complejo molecular tiene una gran influencia en el proceso de síntesis de los materiales híbridos. Cuando el contraión es un haluro el complejo de Au se deposita sobre la superficie del rGO del mismo modo que hacían los complejos de Ru o Ir, dando lugar a especies moleculares bien definidas sobre la superficie del grafeno. Sin embargo, cuando se emplea trifluorometanosulfonato como contraion los resultados cambian completamente. En este caso el complejo de oro no queda depositado sobre la superficie del grafeno, sino que se produce una descomposición controlada del complejo molecular, que da lugar a la formación de nanopartículas funcionalizadas con ligandos NHC, las cuales quedan ancladas sobre el rGO. Este nuevo material híbrido basado en nanopartículas es un catalizador extremadamente eficaz en la hidratación de alquinos, y permite reducir las cargas de catalizador y los tiempos de reacción. Además, el nuevo catalizador es altamente reciclable, pudiéndose reutilizar hasta 10 veces.

En global los resultados obtenidos en la presente tesis doctoral muestran una nueva metodología que permite obtener de manera sencilla nuevos catalizadores sólidos altamente activos y reciclables. 


\subsection{REFERENCIAS}

[1] J. G. De Vries, S. D. Jackson, Catal. Sci. Technol. 2012, 2, 2009.

[2] S. L. Wegener, T. J. Marks, P. C. Stair, Acc. Chem. Res. 2012, 45, 206-214.

[3] A. J. Sandee, L. A. van der Veen, J. N. H. Reek, P. C. J. Kamer, M. Lutz, A. L. Spek, P. W. N. M. van Leeuwen, Angew. Chemie - Int. Ed. 1999, 38, 32313235.

[4] A. J. Sandee, J. N. H. Reek, P. C. J. Kamer, P. W. N. M. Van Leeuwen, J. Am. Chem. Soc. 2001, 123, 8468-8476.

[5] J. Pastva, K. Skowerski, S. J. Czarnocki, N. Žilková, J. Čejka, Z. Bastl, H. Balcar, ACS Catal. 2014, 4, 3227-3236.

[6] W. Wang, L. Cui, P. Sun, L. Shi, C. Yue, F. Li, Chem. Rev. 2018, 118, 9843-9929.

[7] M. R. Axet, O. Dechy-Cabaret, J. Durand, M. Gouygou, P. Serp, Coord. Chem. Rev. 2016, 308, 236-345.

[8] M. R. Axet, J. Durand, M. Gouygou, P. Serp, Adv. Organomet. Chem. 2019, 71, 53-174.

[9] K. S. Novoselov, A. K. Geim, S. V Morozov, D. Jiang, Y. Zhang, S. V. Dubonos, I. V Grigorieva, A. A. Firsov, Science 2004, 306, 666-669.

[10] A. B. Dongil, B. Bachiller-Baeza, A. Guerrero-Ruiz, I. Rodríguez-Ramos, J. Catal. 2011, 282, 299-309.

[11] C. Bai, Q. Zhao, Y. Li, G. Zhang, F. Zhang, X. Fan, Catal. Letters 2014, 144, 1617-1623.

[12] X. Xiong, H. Chen, R. Zhu, Catal. Commun. 2014, 54, 94-99.

[13] S. Verma, M. Aila, S. Kaul, S. L. Jain, RSC Adv. 2014, 4, 30598-30604.

[14] H. P. Mungse, S. Verma, N. Kumar, B. Sain, O. P. Khatri, J. Mater. Chem. 2012, $22,5427-5433$.

[15] A. B. Dongil, B. Bachiller-Baeza, A. Guerrero-Ruiz, I. Rodríguez-Ramos, Catal. Commun. 2012, 26, 149-154.

[16] F. Rao, S. Deng, C. Chen, N. Zhang, Catal. Commun. 2014, 46, 1-5.

[17] M. A. Nasseri, A. Allahresani, H. Raissi, RSC Adv. 2014, 4, 26087-26093. 
[18] Q. Zhao, Y. Li, R. Liu, A. Chen, G. Zhang, F. Zhang, X. Fan, J. Mater. Chem. A 2013, 1, 15039-15045.

[19] V. Georgakilas, M. Otyepka, A. B. Bourlinos, V. Chandra, N. Kim, K. C. Kemp, P. Hobza, R. Zboril, K. S. Kim, Chem. Rev. 2012, 112, 6156-6214.

[20] C. Janiak, J. Chem. Soc. Dalton Trans. 2000, 3885-3896.

[21] F. J. Gómez, R. J. Chen, D. Wang, R. M. Waymouth, H. Dai, Chem. Commun. 2003, 9, 190-191.

[22] G. Liu, B. Wu, J. Zhang, X. Wang, M. Shao, J. Wang, Inorg. Chem. 2009, 48, 2383-2390.

[23] Y. Suzuki, P. Laurino, D. T. Mcquade, P. H. Seeberger, Helv. Chim. Acta 2012, 95, 2578-2588.

[24] L. Zhang, W. Zhang, P. Serp, W. H. Sun, J. Durand, ChemCatChem 2014, 6, 1310-1316.

[25] L. Xing, J. Xie, Y. Chen, L. Wang, Q. Zhou, Adv. Synth. Catal. 2008, 350, 10131016.

[26] C. Vriamont, M. Devillers, O. Riant, S. Hermans, Chem. - A Eur. J. 2013, 19, 12009-12017.

[27] S. Sabater, J. A. Mata, E. Peris, ACS Catal. 2014, 4, 2038-2047.

[28] H. M. J. Wang, I. J. B. Lin, Organometallics 1998, 17, 972-975.

[29] S. Sabater, J. A. Mata, E. Peris, Organometallics 2015, 34, 1186-1190.

[30] E. Balaraman, E. Khaskin, G. Leitus, D. Milstein, Nat. Chem. 2013, 5, 122-125.

[31] G. Tojo, M. I. Fernández, Oxidation of Primary Alcohols to Carboxilic Acids, Springer-Verlag Ney York, 2007.

[32] J. Campos, Phys. Sci. Rev. 2018, 3, 1-25.

[33] F. F. Fleming, Nat. Prod. Rep. 1999, 16, 597-606.

[34] L. Cristian, S. Nica, O. D. Pavel, C. Mihailciuc, V. Almasan, S. M. Coman, V. I. Parvulescu, Catal. Sci. Technol. 2013, 3, 2646-2653.

[35] K. N. T. Tseng, A. M. Rizzi, N. K. Szymczak, J. Am. Chem. Soc. 2013, 135, 16352-16355. 
[36] I. Dutta, S. Yadav, A. Sarbajna, S. De, M. Hölscher, W. Leitner, J. K. Bera, J. Am. Chem. Soc. 2018, 140, 8662-8666.

[37] Z. Wang, J. Belli, C. M. Jensen, Faraday Discuss. 2011, 151, 297-305.

[38] A. Primo, M. Puche, O. D. Pavel, B. Cojocaru, A. Tirsoaga, V. Parvulescu, H. García, Chem. Commun. 2016, 52, 1839-1842.

[39] W. Chen, J. Egly, A. I. Poblador-Bahamonde, A. Maisse-Francois, S. BelleminLaponnaz, T. Achard, Dalton Trans. 2020, 49, 3243-3252.

[40] G. W. Crabtree, M. S. Dresselhaus, M. V Buchanan, G. W. Crabtree, M. S. Dresselhaus, M. V Buchanan, Phys. Today 2004, 57, 1625-1637.

[41] G. Marbán, T. Valdés-solís, Int. J. Hydrogen Energy 2007, 32, 1625-1637.

[42] M. Markiewicz, Y. Q. Zhang, A. Bösmann, N. Brückner, J. Thöming, P. Wasserscheid, S. Stolte, Energy Environ. Sci. 2015, 8, 1035-1045.

[43] K. Sordakis, C. Tang, L. K. Vogt, H. Junge, P. J. Dyson, M. Beller, G. Laurenczy, Chem. Rev. 2018, 118, 372-433.

[44] P. Preuster, C. Papp, P. Wasserscheid, Acc. Chem. Res. 2017, 50, 74-85.

[45] D. Teichmann, W. Arlt, P. Wasserscheid, R. Freymann, Energy Environ. Sci. 2011, 4, 2767-2773.

[46] E. Gianotti, M. Taillades-jacquin, J. Roziere, D. J. Jones, ACS Catal. 2018, 8, 4660-4680.

[47] K. Müller, K. Stark, B. Müller, W. Arlt, Energy Fuels 2012, 26, 3691-3696.

[48] K. Kuciński, G. Hreczycho, ChemCatChem 2017, 9, 1868-1885.

[49] M. Aliaga-Lavrijsen, M. Iglesias, A. Cebollada, K. Garcés, N. García, P. J. Sanz Miguel, F. J. Fernández-Alvarez, J. J. Pérez-Torrente, L. A. Oro, Organometallics 2015, 34, 2378-2385.

[50] D. Ventura-Espinosa, A. Carretero-Cerdán, M. Baya, H. García, J. A. Mata, Chem. - A Eur. J. 2017, 23, 10815-10821.

[51] R. Corberán, M. Sanaú, E. Peris, J. Am. Chem. Soc. 2006, 128, 3974-3979.

[52] D. Ventura-Espinosa, S. Sabater, A. Carretero-Cerdán, M. Baya, J. A. Mata, ACS Catal. 2018, 8, 2558-2566. 
[53] A. Collado, A. Gómez-Suárez, Y. Oonishi, A. M. Z. Slawin, S. P. Nolan, Chem. Commun. 2013, 49, 10745-10747.

[54] M. Michalska, K. Grela, Synlett 2016, 27, 599-603.

[55] M. Gatto, P. Belanzoni, L. Belpassi, L. Biasiolo, A. Del Zotto, F. Tarantelli, D. Zuccaccia, ACS Catal. 2016, 6, 7363-7376.

[56] H. Yin, H. Tang, D. Wang, Y. Gao, Z. Tang, ACS Nano 2012, 6, 8288-8297.

[57] A. Corma, H. Garcia, Chem. Soc. Rev. 2008, 37, 2096-2126. 


